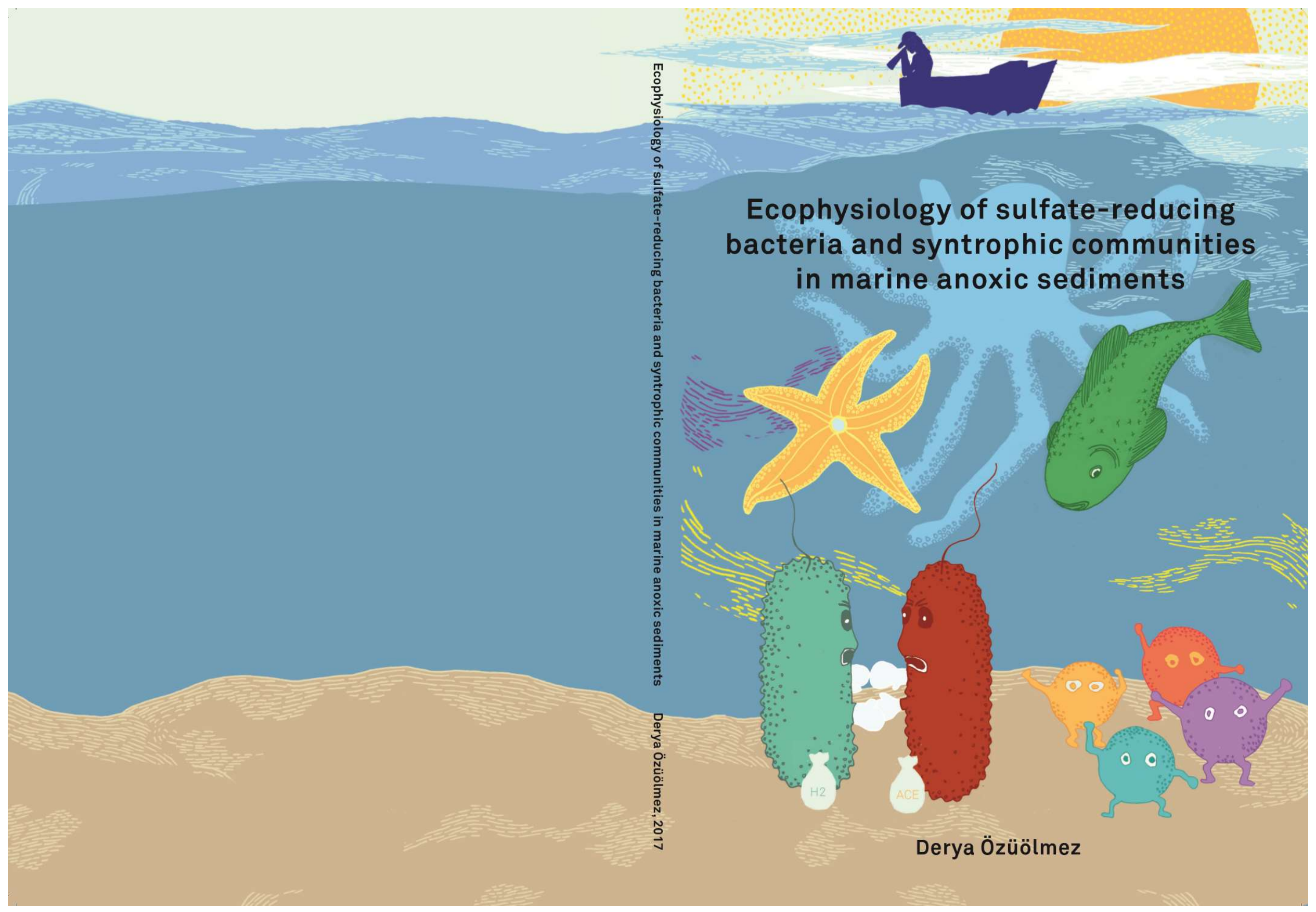




\section{Propositions}

1. Prolonged incubation is a key strategy toward the enrichment of marine syntrophs. (this thesis)

2. Hydrogen-consuming methanogens can effectively compete with hydrogenconsuming sulfate reducers even at high sulfate concentration.

(this thesis)

3. The plastic-eating wax worm, Galleria mellonella, can help cleaning up existing plastic mass (Bombelli et al. 2017, Current Biology 27(8): 292-293), but the real solution to environmental pollution still relies on shifting from disposable plastics to reusable and biodegradable materials.

4. With the discovery of abundant molecular hydrogen in Enceladus' salty ocean (Waite et al. (2017) Science 356:6334, 155-159) extraterrestrial life in our solar system has become highly probable, and should shift our life-hunting focus from distant stars to our nearby neighbors.

5. With the "Help me to do it myself" motto, the Montessori education leads children to grow into mature, creative and self-confident adults.

6. Nothing is impossible as long as the dream is held close to the heart and patience and persistence are applied for the accomplishment.

Propositions belonging to the $\mathrm{PhD}$ thesis entitled:

"Ecophysiology of sulfate-reducing bacteria and syntrophic communities in marine anoxic sediments"

Derya Özüölmez

Wageningen, 12 September 2017 
Ecophysiology of sulfate-reducing bacteria and syntrophic communities in marine anoxic sediments

\author{
Derya Özüölmez
}




\section{Thesis committee}

\section{Promotor}

Prof. Dr Alfons J.M. Stams

Personal chair at the Laboratory of Microbiology

Wageningen University \& Research

\section{Co-promotor}

Dr Caroline M. Plugge

Associate professor, Laboratory of Microbiology

Wageningen University \& Research

\section{Other members}

Prof. Dr Tinka Murk, Wageningen University \& Research

Prof. Dr Gerard Muyzer, University of Amsterdam, The Netherlands

Prof. Dr Stefan Schouten, Utrecht University and Netherlands Institute for Sea Research, Texel, The Netherlands

Dr Verona Vandieken, University of Oldenburg, Germany

This research was conducted under the auspices of the Graduate School for SocioEconomic and Natural Sciences of the Environment (SENSE). 


\title{
Ecophysiology of sulfate-reducing bacteria and syntrophic communities in marine anoxic sediments
}

\author{
Derya Özüölmez
}

\section{Thesis}

submitted in fulfilment of the requirements for the degree of doctor

at Wageningen University

by the authority of the Rector Magnificus,

Prof. Dr A.P.J. Mol,

in the presence of the

Thesis Committee appointed by the Academic Board

to be defended in public

on Tuesday 12 September 2017

at 4 p.m. in the Aula. 


\section{Derya Özüölmez}

Ecophysiology of sulfate-reducing bacteria and syntrophic communities in marine anoxic sediments,

231 pages.

$\mathrm{PhD}$ thesis, Wageningen University, Wageningen, the Netherlands (2017)

With references, with summary in English and Dutch

ISBN: 978-94-6343-654-0

DOI: $10.18174 / 420757$ 


\section{Table of Contents}

$\begin{array}{lll}\text { Chapter } 1 \text { General Introduction } & 1\end{array}$

$\begin{array}{lll}\text { Chapter } 2 & \text { Methanogenic archaea and sulfate reducing bacteria co- } 19\end{array}$ cultured on acetate: teamwork or coexistence?

Chapter 3 Butyrate degradation by sulfate-reducing and methanogenic 45 communities in anoxic sediments of Aarhus Bay, Denmark

Chapter 4 Propionate conversion under sulfidogenic and methanogenic conditions in different biogeochemical zones of Aarhus Bay, Denmark

Chapter 5 Membrane lipid composition in enrichments from the sulfate 119 and methane zones of Aarhus Bay, Denmark

Chapter 6 Physiological and molecular characterization of anaerobic marine propionate- and butyrate-converting syntrophic cultures

Chapter 7 General Discussion

References

Summary

Samenvatting

Author affiliations

Acknowledgements

About the author

List of publications 



\section{Chapter 1}

\section{General Introduction}

\section{Derya Özüölmez}




\subsection{Global carbon cycle}

Carbon is the major element of all life forms, and it is actively cycled in the biosphere. The carbon cycle reflects the flow and exchange of carbon among living organisms and the environment through a series of chemical, physical and biological reactions (Figure 1). Inorganic carbon that is formed from organic matter or weathered from limestone enters the atmosphere as $\mathrm{CO}_{2}$ or in aquatic environments mostly as $\mathrm{HCO}_{3}{ }^{-}$. Atmospheric $\mathrm{CO}_{2}$ is in equilibrium with the carbonate system of the oceans (Takahashi et al., 1997; Fasham et al., 2001). Atmospheric $\mathrm{CO}_{2}$ or $\mathrm{CO}_{2} / \mathrm{HCO}_{3}^{-}$ in the oceans or lakes can be fixed by autotrophic or photolithotrophic organisms (e.g. plants, phytoplankton and marine algae) to synthesize organic carbon. By decay of these organisms, organic carbon is converted back to inorganic carbon through hydrolysis, fermentation and mineralization by the combined action of different organisms (Canfield, 1993; Kristensen and Holmer, 2001).

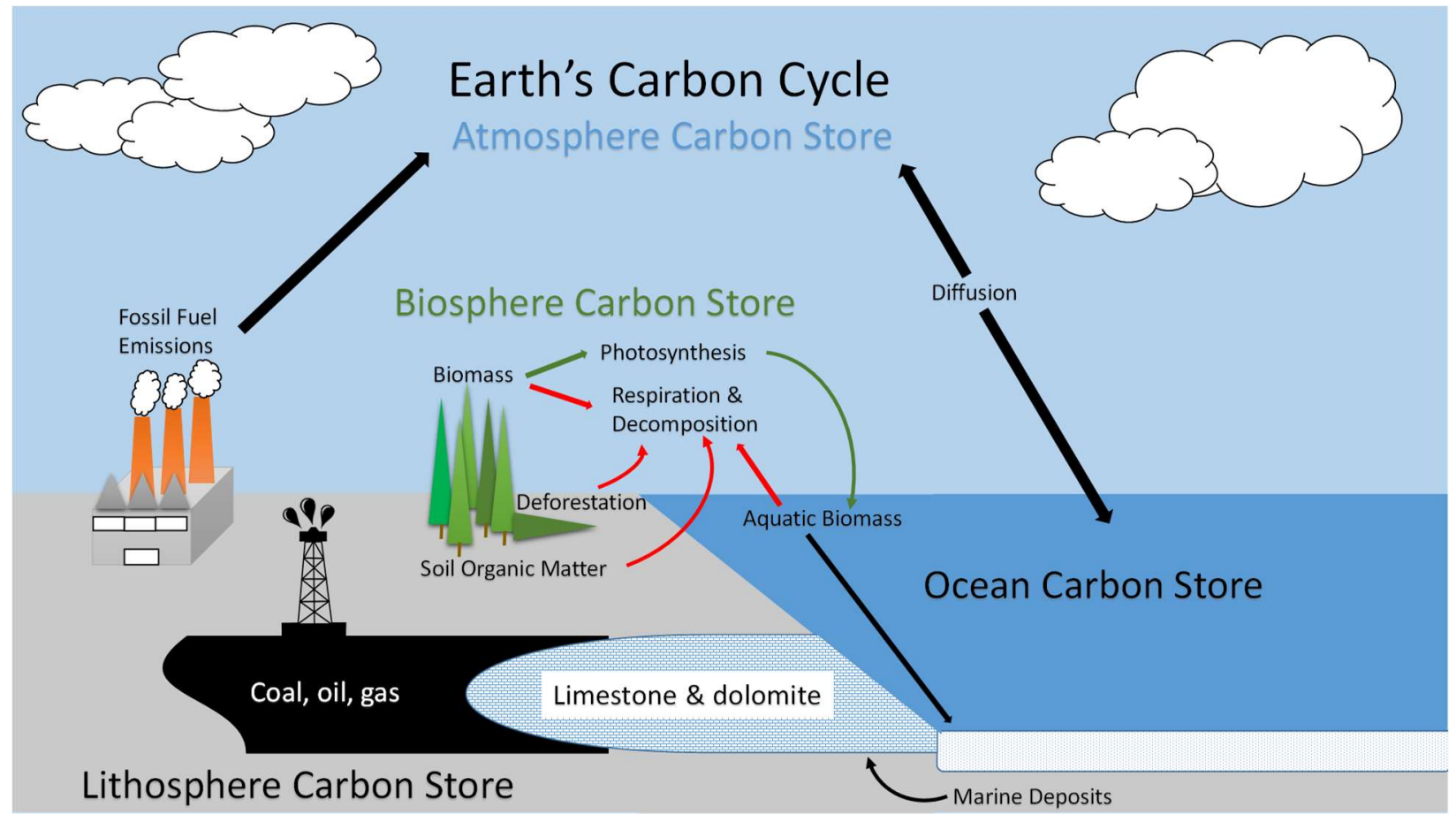

Figure 1. Global carbon cycle. Figure is adapted from Pidwirny, 2012.

Carbon is stored in our planet (a) as living and dead biomass in the biosphere; (b) as carbon dioxide gas in the atmosphere, (c) as organic matter in soils and sediments, (d) in the lithosphere as fossil fuels like coal, oil, and natural gas, and as carbonate-based sedimentary rock deposits such as limestone and dolomite; and (e) in the oceans as dissolved atmospheric carbon dioxide and as 
calcium carbonate shells in marine organisms (Figure 1) (Pidwirny, 2012). The majority of the carbon is fixed as inorganic carbon in limestone or in fossil organic pools, largely as $\mathrm{CO}_{2}$, whereas organic carbon represents only about $0.1 \%$ of the total carbon cycles through the active pool on Earth (Harvey, 2006). Soils represent the largest pool within this active cycle. This is followed by land biota, dissolved organic matter in seawater, and surficial marine sediments with decreasing amounts of organic matter. The smallest fraction includes marine biota and particulate pools (Harvey, 2006). Although the particulate organic carbon reservoir is small, it is dynamic and plays a central role in both amount and composition of organic matter which reaches underlying sediments (Harvey, 2006).

\subsection{Degradation of organic matter in marine sediments}

Coastal marine ecosystems receive regular inputs of organic matter and nutrients from primary production of phytoplankton, rivers, coastal erosion, and the atmosphere (Jørgensen, 1982). Mineralization of the particulate organic matter starts already in the water column. On the other hand, the organic matter that is not decomposed in the water column rapidly sinks down to the sediment in coastal shelf sediments and part of it gets buried in the sediment (Jørgensen, 1983). It is shown that $10-50 \%$ of carbon from primary production was deposited on the bottom in coastal shelf sediments (o-20o $\mathrm{m}$ depth), whereas this fraction decreases to about $1 \%$ in pelagic sediments (500o-60oo m) (Jørgensen, 1983). The coastal shelfs comprise $8.6 \%$ of the total area of the oceans, and it is estimated that $83 \%$ of the mineralization in the sea bottom takes place in these shelf areas (Jørgensen, 1983). This reflects the importance of coastal and shelf areas in the carbon cycle.

High microbial activity in the upper sediment layers leads the formation of distinct biogeochemical zones. The depth range of each zone varies strongly depending on the supply of organic matter from overlying seawater and the sedimentation rate which have an effect on accumulation of organic matter (Jørgensen, 1983). In coastal marine sediments, the oxic surface layer constitutes a thin layer due to the rapid consumption of oxygen for aerobic mineralization. Therefore, the remaining organic matter will be degraded by anaerobic microbes and part of it will be buried in the sediment where mineralization continues albeit slow. In the anoxic part of the sediment, nitrate, manganese, iron, sulfate and carbon dioxide serve as terminal electron acceptors for the mineralization processes (Figure 2). The depth sequence of electron acceptors reflects a gradual decrease in redox potential and thus a decrease in the free energy available by respiration. 
It is estimated that $25-50 \%$ of the organic carbon is mineralized through sulfate reduction, which makes sulfate an important electron acceptor in anoxic part of the sediments (Jørgensen, 1982).

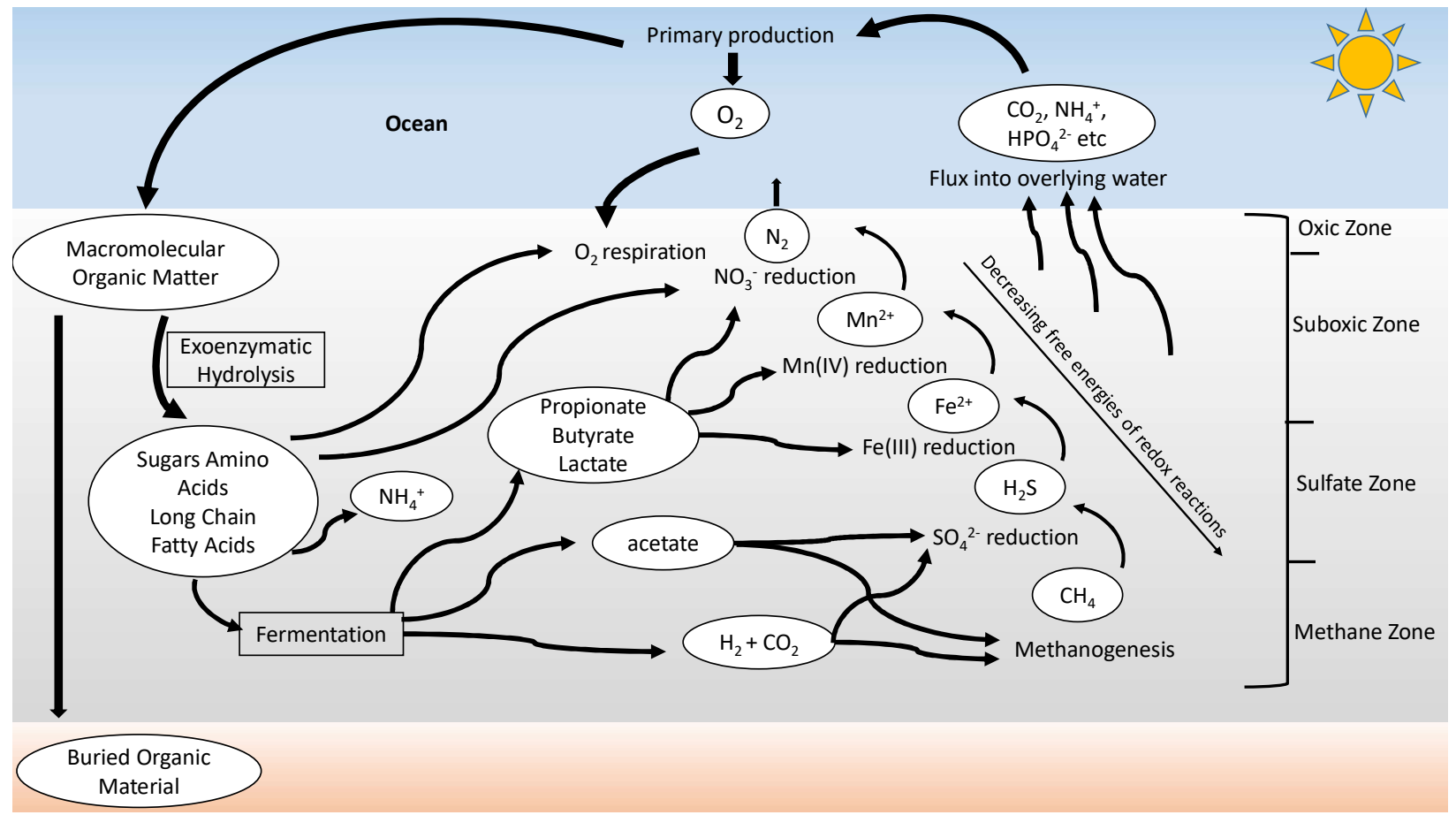

Figure 2. Organic carbon degradation pathways in marine sediments and their relation to the biological redox zonation. Figure is adapted from Parkes et al., 2014 and Jørgensen, 2006.

Anaerobic degradation of organic matter in marine sediments is a complex sequential process in which a variety of physiologically different microorganisms takes part. The first step is an extracellular hydrolysis of polymers (polysaccharides, proteins, nucleic acids and lipids) (Fig 2, Fig 3). Primary fermenting bacteria ferment the monomers and oligomers to fatty acids, branchedchain fatty acids, alcohols, aromatic acids, $\mathrm{H}_{2}$ and $\mathrm{CO}_{2}$. Some of these fermentation products, such as acetate, $\mathrm{H}_{2}, \mathrm{CO}_{2}$, and other one-carbon compounds, can be converted directly to methane and carbon dioxide by methanogens. In methanogenic environments, secondary fermenters or proton reducers convert alcohols, long-chain, branched-chain and aromatic fatty acids to acetate, formate, $\mathrm{H}_{2}$ and $\mathrm{CO}_{2}$ which are then used by the methanogens (Figure 3b) (Schink and Stams, 2013). The conversion of polymers in sulfate-rich anoxic habitats such as marine sediments is slightly different. Polymers are degraded by primary fermenting bacteria and fermentation products are formed. Different from methanogens, sulfate-reducing bacteria can use all products of primary fermentations, and oxidize them to carbon dioxide, while reducing sulfate to sulfide (Figure za) (Widdel 1988). 
a

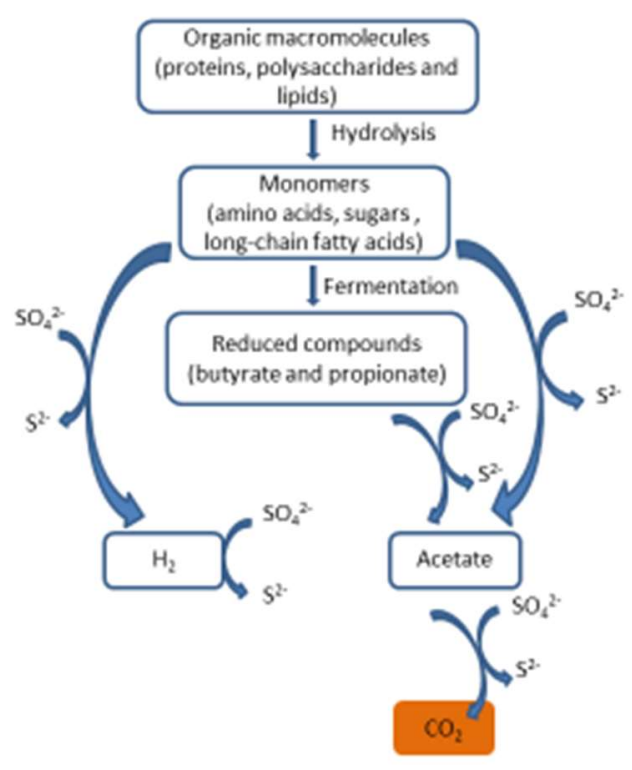

b

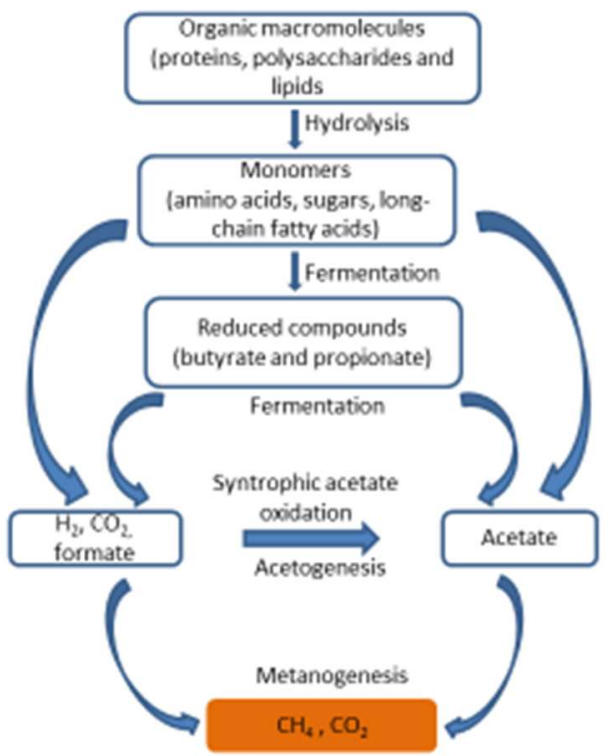

Figure 3. Mineralization of organic matter in the presence (a) and absence (b) of sulfate in anoxic marine sediments. Figure is adapted from Muyzer and Stams, 2008.

Sulfate reduction and methanogenesis are the terminal steps in the degradation process (Jørgensen, 1982; Schink and Stams, 2013; Muyzer and Stams, 2008; Stams and Plugge, 2009). They are thought to be mutually exclusive in most environmental settings and controlled mainly by the amount of available sulfate (Reeburgh and Heggie, 1977; Winfrey and Zeikus, 1977).

\subsection{Microbial interactions shaping biogeochemical zonation}

Previous studies on marine sediments have explained the biological redox zonation by competition among different physiological groups (Martens and Berner 1974; Lovley and Goodwin 1988; Chapelle and Lovley 1992; Hoehler et al., 1998). Accordingly, methanogenesis and sulfate reduction were suggested to be temporally or spatially separated (Cappenberg, 1974; Mountfort and Asher, 1979) and methanogenesis is typically dominant in deeper sulfate-depleted sediment parts that are below the active sulfate reduction zone. Thus, when different physiological groups compete for a common substrate, the microbes using the energetically most favorable electron acceptor available will outcompete the ones using energetically less favorable electron acceptors. For instance, methanogenic Archaea do not utilize large organic molecules but can use only a narrow spectrum of substrates. Most methanogens produce methane either by reducing $\mathrm{CO}_{2}$, with $\mathrm{H}_{2}$ as the primary 
electron donor, or by cleaving acetate. However, sulfate reducers outcompete methanogens for the common substrates, $\mathrm{H}_{2}$ and acetate, due to their higher affinity and lower threshold values for these substrates (Stams, 1994; Hoehler et al., 1998; Muyzer and Stams, 2008) (Table 1). Consequently, significant methane generation in marine sediments occurs at deeper sediment zones, after almost all sulfate has been reduced to sulfide (Jørgensen and Kasten, 2006). The third substrate group for methanogens includes methylated compounds such as methanol, methylated amines (mono-, di-, and trimethylamine, and tetramethylammonium), and methylated sulfides (e.g., dimethyl sulfide and methanethiol). These compounds are known as non-competitive substrates since they are not commonly utilized by sulfate reducers, but mainly by methylotrophic methanogens (Liu and Whitman, 2008). The consumption of non-competitive substrates by methanogens allow simultaneous methanogenesis and sulfate reduction within sulfate containing anoxic sediments, as demonstrated in several investigations (Oremland and Taylor, 1978; Oremland and Polcin, 1982; Oremland et al., 1982a, 1982b, 1987; Kiene et al., 1986; Oremland et al., 1988; Wang and Lee, 1995). On the other hand, methanogenesis from $\mathrm{H}_{2}$ and acetate was also observed within the sulfate zones (Wellsbury et al., 2002; Parkes et al., 2005). Therefore, there is no distinct separation of sulfate reducers and methanogens along the sediment, and the absence of methane in sulfate zones might indicate the occurrence of active anaerobic oxidation of methane (AOM) (Parkes et al., 2014).

The sulfate-methane transition zone (SMTZ) corresponds to the sedimentary interval characterized by a mutual depletion of methane and sulfate due to the microbial anaerobic oxidation of methane (Sultan et al., 2016; Iversen and Jørgensen, 1985). Previously, it was postulated that sulfate and methane profiles at this specific part of the sediment were formed due to competition of sulfate reducers and methanogens for common substrates (Martens and Berner, 1974). After several studies, however, it was demonstrated that methane, which diffuses upwards from the deeper sediment, is oxidized by anaerobic methanotrophic archaea (ANME) coupled to sulfate reduction (Barnes and Goldberg, 1976; Martens and Berner, 1977; Murray et al., 1978; Reeburgh, 1980; Alperin et al., 1988; Hinrichs et al., 1999; Krüger et al 2003; Nauhaus et al 2005). Later, in vitro studies showed that AOM and sulfate reduction are directly coupled and this is a syntrophic processes involving ANME and SRB that are metabolically interdependent on each other (Nauhaus et al., 2002; Nauhaus et al., 2005). The depth of the transition zone varies depending on the organic matter supply, the depth of the methane production zone, and the transport and consumption rates of methane and sulfate (Claypool and Kvenvolden, 1983; Borowski et al., 1999; Claypool, 2004; Jørgensen and Kasten, 2006; Knittel and Boetius, 2009). 


\subsection{Syntrophy}

Methanogenic degradation of organic matter in sulfate-depleted marine sediments is carried out by the cooperation of physiologically different microorganisms. This relationship is called 'syntrophy' and involves the consumption of degradation end products, usually hydrogen, formate and acetate, by the partner organisms. Syntrophic bacteria perform secondary fermentation of small organic molecules such as lactate, propionate, butyrate and produce acetate, formate, $\mathrm{H}_{2}$ and $\mathrm{CO}_{2}$ (Morris et al., 2013; Schink and Stams, 2013). However, these reactions are endergonic under standard conditions and thus not possible unless a $\mathrm{H}_{2}$ /formate scavenging partner organism keeps the concentration of these intermediates low (Schink and Stams, 2013). Hydrogenotrophic methanogens act as syntrophic partners of syntrophs by utilizing $\mathrm{H}_{2}$ and formate to form $\mathrm{CH}_{4}$ (Morris et al., 2013; Sieber et al., 2014) (Figure 4). This anaerobic metabolism, especially when methanogenesis is the terminal electron accepting process, involves consortia with tightly coupled syntrophic partnerships (Schink and Stams, 2013; McInerney et al., 2008). Syntrophic interactions are not restricted to the methanogenic environments but also occur in sulfate-reducing environments as evidenced by sulfate-reducing consortia involved in anaerobic oxidation of methane (AOM) (Nauhaus et al., 2005) (Figure 4).

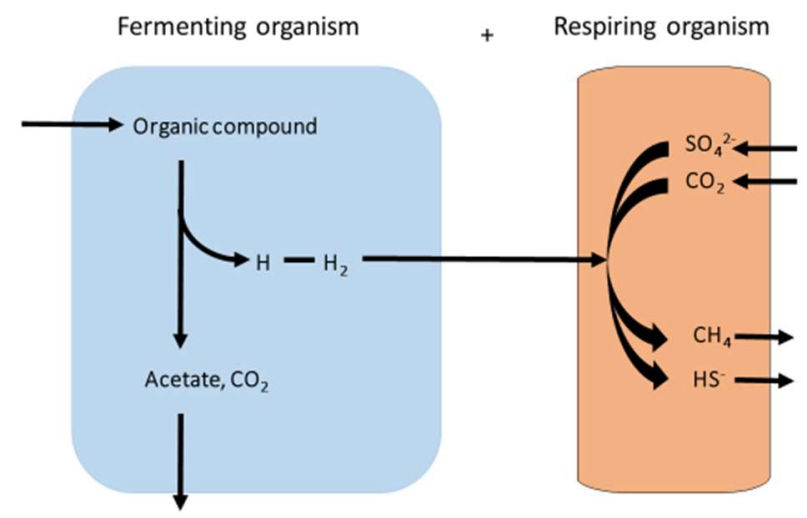

Figure 4. Schematic diagram of interspecies hydrogen transfer between fermenting and respiring organism.

It has also been demonstrated that syntrophic consortia of acetogens and hydrogen-/formateconsuming sulfate reducers catalyze propionate and butyrate degradation in sulfate-reducing environments (Stams et al., 2005; Elferink et al., 1998; Visser et al., 1993). It is not surprising that hydrogen-/formate-consuming sulfate reducers are commonly used as the syntrophic partner to 
isolate syntrophic propionate- and butyrate-converting microorganisms in co-cultures (Dong et al., 1994; Boone and Bryant, 1980; McInerney et al., 1979).

Interspecies electron transfer plays a key role in the functioning of methanogenic microbial communities, which have a significant impact on the global carbon cycle (Stams and Plugge, 2009; Sieber et al., 2012). Therefore, it is crucial to study the syntrophic cooperations to understand methanogenic conversions in different environments (McInerney et al., 2008). The most difficult step in the methanogenic degradation is the second fermentation step in which short-chain fatty acids such as propionate and butyrate are converted. The oxidation of propionate and butyrate to $\mathrm{H}_{2}$, formate and acetate are endergonic reactions under standard conditions $\left(\mathrm{P}_{\mathrm{H}_{2}}\right.$ of 1 atm, substrate and product concentrations of $1 \mathrm{M}$, temperature $298^{\circ} \mathrm{K}, \mathrm{pH} 7$ ) (Table 1 ).

Table 1. Equations and standard free energy changes for acetate and hydrogen producing and methanogenic reactions at standard conditions (at $1 \mathrm{M}, \mathrm{pH} 7.0,1 \mathrm{~atm}$ and $\mathrm{T}=25^{\circ} \mathrm{C}$ ). $\Delta \mathrm{G}^{\mathrm{o}^{\prime}}$ values taken from Thauer et al., 1977.

\begin{tabular}{|c|c|c|c|}
\hline \multirow{2}{*}{$\begin{array}{l}\text { Equation } \\
\text { Sulfate-reducing reactions }\end{array}$} & \multicolumn{3}{|c|}{$\Delta \mathrm{G}^{\circ 1}$ (kJ/reaction) } \\
\hline & & & \\
\hline Butyrate $^{-}+0.5 \mathrm{SO}_{4}{ }^{2-}$ & $\rightarrow$ & 2 Acetate $^{-}+0.5 \mathrm{HS}^{-}+0.5 \mathrm{H}^{+}$ & -27.8 \\
\hline Propionate -0.75 & $\rightarrow$ & Acetate ${ }^{-}+0.75 \mathrm{HS}^{-}+\mathrm{HCO}_{3}^{-}+0.25 \mathrm{H}^{+}$ & -37.8 \\
\hline Acetate $^{-}+\mathrm{SO}_{4}^{2-}$ & $\rightarrow$ & $2 \mathrm{HCO}_{3}^{-}+\mathrm{HS}^{-}$ & -47.6 \\
\hline $4 \mathrm{H}_{2}+\mathrm{SO}_{4}^{2-}+\mathrm{H}^{+}$ & $\rightarrow$ & $\mathrm{HS}^{-}+4 \mathrm{H}_{2} \mathrm{O}$ & -151.9 \\
\hline Formate ${ }^{-}+\mathrm{SO}_{4}{ }^{2-}+\mathrm{H}^{+}$ & $\rightarrow$ & $4 \mathrm{HCO}_{3}^{-}+\mathrm{HS}^{-}$ & -146.7 \\
\hline \multicolumn{4}{|l|}{ Hydrogen-producing reactions } \\
\hline Propionate $^{-}+2 \mathrm{H}_{2} \mathrm{O}$ & $\rightarrow$ & Acetate ${ }^{-}+\mathrm{CO}_{2}+3 \mathrm{H}_{2}$ & +76.0 \\
\hline Propionate $^{-}+2 \mathrm{H}_{2} \mathrm{O}+2 \mathrm{CO}_{2}$ & $\rightarrow$ & Acetate $^{-}+3 \mathrm{HCOO}^{-}+3 \mathrm{H}^{+}$ & +65.3 \\
\hline Butyrate $^{-}+2 \mathrm{H}_{2} \mathrm{O}$ & $\rightarrow$ & 2 Acetate $^{-}+\mathrm{H}^{+}+2 \mathrm{H}_{2}$ & +48.3 \\
\hline Butyrate $^{-}+2 \mathrm{H}_{2} \mathrm{O}+2 \mathrm{CO}_{2}$ & $\rightarrow$ & 2 Acetate $^{-}+2 \mathrm{HCOO}^{-}+2 \mathrm{H}^{+}$ & +38.5 \\
\hline Acetate $^{-}+4 \mathrm{H}_{2} \mathrm{O}$ & $\rightarrow$ & $4 \mathrm{H}_{2}+2 \mathrm{HCO}_{3}^{-}+\mathrm{H}^{+}$ & +104.6 \\
\hline Formate $^{-}+\mathrm{H}_{2} \mathrm{O}$ & $\rightarrow$ & $\mathrm{HCO}_{3}^{-}+\mathrm{H}_{2}$ & +1.3 \\
\hline \multicolumn{4}{|l|}{ Methanogenic reactions } \\
\hline $4 \mathrm{H}_{2}+\mathrm{HCO}_{3}^{-}+\mathrm{H}^{+}$ & $\rightarrow$ & $\mathrm{CH}_{4}+3 \mathrm{H}_{2} \mathrm{O}$ & -135.6 \\
\hline Acetate $^{-}+\mathrm{H}_{2} \mathrm{O}$ & $\rightarrow$ & $\mathrm{CH}_{4}+\mathrm{HCO}_{3}^{-}$ & -31.0 \\
\hline Formate $^{-}+\mathrm{H}^{+}+3 \mathrm{H}_{2}$ & $\rightarrow$ & $\mathrm{CH}_{4}+2 \mathrm{H}_{2} \mathrm{O}$ & -134.3 \\
\hline
\end{tabular}

\subsection{Syntrophic butyrate conversion}

All known syntrophic butyrate-degrading bacteria belong to the phyla Firmicutes and Proteobacteria (Table 2). Besides oxidation of saturated fatty acids in coculture with methanogens, most butyrate degraders can grow axenically only by fermenting unsaturated fatty acids such as 
crotonate (Schink, 1997). Butyrate degraders use the beta-oxidation pathway to oxidize butyrate (Schink, 1997) (Figure 5A). Thermodynamically the most difficult step in butyrate oxidation is the oxidation of butyryl-CoA to crotonyl-CoA. Hydrogen (at $1 \mathrm{~Pa}_{2}$ ) and formate (at $1 \mathrm{mM}$ formate) (the minimum level that can be maintained by methanogens) production from electrons generated in the oxidation of butyryl-CoA to crotonyl-CoA is endergonic. Therefore, a process called 'reversed electron transport' is required to use part of the gained ATP to drive these redox reactions (Sieber et al., 2012; McInerney et al., 2009). Genomic analysis indicates that several different gene clusters are involved in syntrophic reverse electron transfer (Sieber et al., 2012). Müller et al. (2009) partially purified an NADH:acceptor oxidoreductase from syntrophically grown $S$. wolfei cells and proposed that this enzyme complex is homologue of the Thermotoga maritima bifurcating [FeFe]hydrogenase and are involved in the thermodynamically favorable production of hydrogen from reduced ferredoxin to drive the unfavorable production of hydrogen from NADH by a process called electron confurcation (Müller et al. 2010; Schut and Adams, 2009). Since this enzyme complex has subunits predicted to function as an $\mathrm{NADH}$-linked formate dehydrogenase and an NADH-linked hydrogenase, interspecies electron transfer may occur via either hydrogen and/or formate, depending on the environmental conditions (Müller et al. 2009; Müller et al. 2010). However, it needs to be verified if the hydrogenase acts in a bifurcating manner as observed in T. maritima. A possible mechanism for reverse electron transfer is electron bifurcation by butyryl-CoA dehydrogenase:electron transfer flavoprotein (bcd/etfAB) complex of Clostridium kluyveri (Li et al., 2008). Syntrophomonas wolfei has a gene cluster homologous to the bcd/etfAB complex from $C$. kluyveri (Sieber et al., 2010). Previous studies showed that bifurcation of electrons from NADH with crotonyl-CoA and oxidized ferrodoxin by the bcd/etfAB complex of $C$. kluyveri is not involved in butyrate oxidation by S.wolfei, as Bcd of S. wolfei is not associated with etfAB (Sieber et al., 2012; Müller et al. 2010; Müller et al. 2009). Ion gradients can also drive the reverse electron transfer during syntrophic metabolism (McInerney et al., 2009). The ferredoxin:NAD ${ }^{+}$oxidoreductase may function as a reverse electron transfer complex, using the ion gradient to drive the unfavorable reduction of ferredoxin with NADH (Sieber et al., 2012). The genome of $S$. wolfei contains a gene for a membrane-bound iron-sulfur (FeS) oxidoreductase that is adjacent to the two genes for electron transfer flavoprotein (etfAB) (Callaghan et al., 2011; Sieber et al., 2010; McInerney et al., 2007). The FeS complex could funnel electrons from $\beta$-oxidation to membrane redox carriers (Sieber et al., 2010; McInerney et al., 2007). 
Table 2. Butyrate and propionate degrading syntrophs and the substrates that they utilize in pure culture and in syntrophic cultures. Adapted from Schink and Stams, 2013 and Sieber et al., 2012.

\begin{tabular}{|c|c|c|c|c|c|c|}
\hline \multirow{2}{*}{ Organism } & \multirow{2}{*}{ pH range } & \multirow{2}{*}{$\begin{array}{c}\text { Temperature } \\
\text { range }\left({ }^{\circ} \mathrm{C}\right)\end{array}$} & \multicolumn{2}{|c|}{ Substrates used } & \multirow{2}{*}{$\begin{array}{l}\text { Phylogenetic } \\
\text { affiliation }\end{array}$} & \multirow{2}{*}{ References } \\
\hline & & & Pure culture & Syntrophic coculture & & \\
\hline \multicolumn{7}{|l|}{ Butyrate degraders } \\
\hline Syntrophomonas wolfei & ND & $35-37$ & Crotonate & $\mathrm{C} 4-\mathrm{C}^{\mathrm{a}}$ & Firmicutes & $\begin{array}{l}\text { Mclnerney et al. } \\
(1979,1981)\end{array}$ \\
\hline Syntrophomonas sapovorans & $6.3-8.1$ & $25-45$ & None & C4-C18 & Firmicutes & Roy et al. (1986) \\
\hline $\begin{array}{l}\text { Syntrophomonas } \\
\text { (Syntrophospora) bryantii }\end{array}$ & $6.5-7.5$ & $28-34$ & Crotonate & $\begin{array}{l}\text { C4-C11, 2-methyl } \\
\text { valerate }\end{array}$ & Firmicutes & $\begin{array}{l}\text { Stieb and Schink } \\
\text { (1985); Zhao et al. } \\
(1989)\end{array}$ \\
\hline Syntrophomonas curvata & $6.3-8.4$ & $20-42$ & Crotonate & C4-C18, C18:1 & Firmicutes & Zhang et al. (2004) \\
\hline Syntrophomonas erecta & $6.0-8.8$ & $25-47$ & Crotonate & C4-C8 & Firmicutes & Zhang et al. (2005) \\
\hline Syntrophomonas zehnderi & 7.0 & $25-40$ & None & $\begin{array}{l}\text { C4-C18, C16:1, C18:1, } \\
\text { C18:2 }\end{array}$ & Firmicutes & $\begin{array}{l}\text { Sousa et al. } \\
\text { (2007a) }\end{array}$ \\
\hline Thermosyntropha lipolytica & $7.15-9.5$ & $52-70$ & \begin{tabular}{|l|} 
Crotonate, yeast \\
extract, tryptone, \\
casamino acids, \\
betaine, \\
pyruvate, ribose, \\
xylose
\end{tabular} & $\begin{array}{l}\text { C4-C18, C18:1, C18:2; } \\
\text { triglycerides }\end{array}$ & Firmicutes & $\begin{array}{l}\text { Svetlitshnyi et al. } \\
\text { (1996) }\end{array}$ \\
\hline Syntrophothermus lipocalidus & $5.8-7.5$ & $45-60$ & Crotonate & C4-C10, isobutyrate & Firmicutes & $\begin{array}{l}\text { Sekiguchi et al } \\
(2000)\end{array}$ \\
\hline Syntrophus aciditrophicus & ND & $25-42$ & Crotonate & $\begin{array}{l}\text { Butyrate, benzoate, } \\
\text { alicyclic compounds, } \\
\text { fatty acids }\end{array}$ & 8-Proteobacteria & $\begin{array}{l}\text { Jackson et al., } \\
1999\end{array}$ \\
\hline Algorimarina butyrica & $6.2-7.1$ & $10-25$ & None & C4, isobutyrate & 8-Proteobacteria & \begin{tabular}{|l} 
Kendall et al. \\
$(2006)$
\end{tabular} \\
\hline \multicolumn{7}{|l|}{ Propionate degraders } \\
\hline Syntrophobacter wolinii & ND & ND & $\begin{array}{l}\text { Propionate with } \\
\text { sulfate; fumarate }\end{array}$ & Propionate & 8-Proteobacteria & $\begin{array}{l}\text { Boone and Bryant } \\
\text { (1980), } \\
\text { Wallrabenstein et } \\
\text { al. (1994) }\end{array}$ \\
\hline $\begin{array}{l}\text { Syntrophobacter } \\
\text { fumaroxidans }\end{array}$ & $6.0-8.0$ & $20-40$ & \begin{tabular}{|l|} 
Propionate, \\
formate, \\
hydrogen with \\
sulfate and \\
fumarate; \\
Succinate with \\
sulfate; fumarate, \\
malate, \\
aspartate, \\
pyruvate,
\end{tabular} & Propionate & 8-Proteobacteria & $\begin{array}{l}\text { Harmsen et al. } \\
\text { (1998) }\end{array}$ \\
\hline Syntrophobacter pfennigii & $6.2-8.0$ & $30-37$ & \begin{tabular}{|l} 
Propionate, \\
lactate with \\
sulfate, sulfite, \\
thiosulfate \\
\end{tabular} & $\begin{array}{l}\text { Propionate, lactate, } \\
\text { propanol }\end{array}$ & $\delta$-Proteobacteria & $\begin{array}{l}\text { Wallrabenstein et } \\
\text { al. (1995) }\end{array}$ \\
\hline $\begin{array}{l}\text { Syntrophobacter } \\
\text { sulfatireducens }\end{array}$ & $6.2-8.8$ & $20-48$ & $\begin{array}{l}\text { Propionate with } \\
\text { sulfate or } \\
\text { thiosulfate; } \\
\text { pyruvate } \\
\end{array}$ & Propionate & $\delta$-Proteobacteria & Chen et al. (2005) \\
\hline $\begin{array}{l}\text { Pelotomaculum } \\
\text { thermopropionicum }\end{array}$ & $6.7-7.5$ & $45-65$ & \begin{tabular}{|l|} 
Pyruvate, \\
fumarate; \\
propionate, \\
ethanol or lactate \\
with fumarate
\end{tabular} & $\begin{array}{l}\text { Propionate, ethanol, } \\
\text { lactate, ethylene } \\
\text { glycol, 1-butanol, 1- } \\
\text { propanol, 1-pentanol } \\
\text { and 1,3-propanediol }\end{array}$ & Firmicutes & $\begin{array}{l}\text { Imachi et al. } \\
(2002)\end{array}$ \\
\hline
\end{tabular}




\begin{tabular}{|l|c|c|l|l|l|l|}
\hline Pelotomaculum schinkii & ND & ND & None & Propionate & Firmicutes & $\begin{array}{l}\text { de Bok et al. } \\
\text { (2002a) }\end{array}$ \\
\hline Smithella propionica & ND & ND & Crotonate & Propionate & 8-Proteobacteria & Liu et al. (1999) \\
\hline $\begin{array}{l}\text { Desulfotomaculum } \\
\text { thermobenzoicum subsp. } \\
\text { thermosyntrophicum }\end{array}$ & $6.0-8.0$ & $45-62$ & $\begin{array}{l}\text { propionate, } \\
\text { lactate, pyruvate } \\
\text { and } \mathrm{H}_{2} / \mathrm{CO}_{2} \text { with } \\
\text { sulfate; Pyruvate, } \\
\text { lactate, fumarate, } \\
\text { glycine, benzoate }\end{array}$ & Propionate & Firmicutes \\
\hline
\end{tabular}

a The number of carbons in the fatty acid is indicated. When a range is given, this means that the organism can use compounds within the indicated range of carbon numbers, but not all possibilities were tested. ND = Not determined.

Recently, Schmidt et al. (2013) confirmed the constitutive expression of a membrane-bound, internally oriented iron-sulfur oxidoreductase (DUF224) and electron-transfer flavoproteins (etfAB) during syntrophic butyrate oxidation by S. wolfei. According to Schmidt et al. (2013), electrons released in the butyryl-CoA dehydrogenase reaction are transferred through a membranebound EtfAB:quinone oxidoreductase (DUF224) to a menaquinone and further via a b-type cytochrome to an externally oriented formate dehydrogenase. Hence, an ATP hydrolysis driven proton-motive force across the cytoplasmatic membrane would provide the energy input for the electron potential shift necessary for formate formation (Schmidt et al., 2013). Thus, formate would be the preferential electron carrier in syntrophic butyrate oxidation by S. wolfei, or could be exchanged into hydrogen as electron carrier at the hydrogenase/formate dehydrogenase (HYD1/FDH-1) complex (Schmidt et al., 2013). In the 3-hydroxybutyryl-CoA dehydrogenase reaction, the second reaction that generates electrons (NADH), electrons are transferred to $\mathrm{NAD}^{+}$to form $\mathrm{NADH}$. The NADH can then be used to reduce either protons to molecular hydrogen, or $\mathrm{CO}_{2}$ to formate (Schmidt et al., 2013). 



Figure 5. Metabolic pathways of butyrate (A) and propionate (B) conversion by bacteria that can grow in syntrophy with methanogens.

\subsection{Syntrophic propionate conversion}

Syntrophic propionate degraders are affiliated with the classes of Deltaproteobacteria and Clostridia (Imachi et al., 2002; Plugge et al., 2002) (Table 2). Different from syntrophic butyrate degraders, some syntrophic propionate degraders, such as Syntrophobacter sp., can grow in pure culture by using sulfate as electron acceptor for propionate oxidation (McInerney et al., 2005). Additionally, some can grow by fermenting pyruvate or fumarate in pure culture. Smithella propionica ferments propionate via dismutating pathway to acetate and butyrate, and butyrate is subsequently oxidized to acetate (de Bok et al., 2001). All other known syntrophic propionate degraders use methymalonyl-CoA pathway to oxidize propionate to acetate and $\mathrm{CO}_{2}$ (Figure ${ }_{5} \mathrm{~B}$ ). The key redox reactions are the oxidation of succinate to fumarate, malate to oxaloacetate and the conversion of pyruvate to acetyl-CoA and $\mathrm{CO}_{2}$ (Schink, 1997). Oxidation of succinate and malate are highly endergonic reactions and thus require an energy input via reverse electron transfer. The reverse electron transport for the oxidation of succinate during syntrophic propionate metabolism involves coupling menaquinone reduction with the oxidation of succinate to fumarate by succinate dehydrogenase. In Syntrophobacter fumaroxidans and Pelotomaculum thermopropionicum, menaquinone oxidation is linked to a membrane-bound hydrogenase or formate dehydrogenase 
(Sieber et al., 2012). S. fumaroxidans has a membrane-bound succinate dehydrogenase, two cytoplasmic succinate dehydrogenases, and several periplasmic and cytoplasmic hydrogenases and formate dehydrogenases (Müller et al., 2010; Worm et al., 2011; de Bok et al., 2002a, 2003). Significantly higher expression of the confurcating hydrogenase, a periplasmic formate dehydrogenase, and the hydrogen-formate lyase was observed during syntrophic growth versus monoculture growth of $S$. fumaroxidans (Worm et al, 2011). P. thermopropionicum also contains a similar system with a membrane-associated succinate dehydrogenase, which was transcribed highly during coculture growth on propionate (Kato et al., 2009). Malate oxidation to oxaloacetate and pyruvate to acetyl-CoA and $\mathrm{CO}_{2}$ also generates each two electrons (NADH) (van Kuijk et al., 1996; Chabrière et al., 1999). Genome analysis suggests that NADH generated from malate oxidation and reduced ferredoxin generated from pyruvate oxidation could be coupled to formate or hydrogen production by confurcating formate dehydrogenases and hydrogenases (Müller et al. 2010). Such a mechanism would use the energy that remains from ferredoxin oxidation with protons to allow the endergonic coupling of NADH oxidation to proton reduction.

Among multiple routes that have been suggested for reverse electron transfer mechanism during syntrophic metabolism, the presence of confurcating-type hydrogenase genes and a gene for novel membrane-bound FeS oxidoreductase and adjacent to etfAB were found to be common in almost all microorganisms capable of syntrophic metabolism, despite the distinctly different phylogenetic lineages of these organisms (Sieber et al., 2012).

\subsection{Do sulfate reducers and syntrophs coexist throughout the sediment?}

In anoxic marine environments where the amount of organic carbon is high, both sulfate reduction and methanogenesis were reported to occur simultaneously (Oremland and Taylor, 1978; Oremland et al., 1982b; Senior et al., 1982; Holmer et al., 1994). There are several studies supporting the coexistence of both microbial groups using competitive substrates, $\mathrm{H}_{2}$ and acetate, in sulfate-rich sediments (Oremland and Taylor, 1978; Senior et al., 1982).

The zonation of mineralization processes may be kinetically or energetically controlled based on the abilities of the physiological groups to compete for common substrates. The $\mathrm{H}_{2}$ concentration in anaerobic sedimentary environments is an important factor in determining the predominant terminal electron-accepting process and consequently the biological redox zonation observed in sediments. According to Lovley and Goodwin (1988), each terminal electron-accepting process requires different minimum $\mathrm{H}_{2}$ concentrations in order to conserve energy and to grow. 
They reported the $\mathrm{H}_{2}$ concentration range for methanogenesis as $7-10 \mathrm{nM}$, for sulfate reduction as $1-1.5 \mathrm{nM}$, for Fe (III) reduction as $0.2 \mathrm{nM}$, and for $\mathrm{Mn}(\mathrm{IV}) /$ nitrate reduction as less than $0.05 \mathrm{nM}$ (Lovley and Goodwin, 1988). Hence, sulfate reducers will maintain $\mathrm{H}_{2}$ concentrations at very low levels $(1-1.5 \mathrm{nM})$ in sulfate zones, and $\mathrm{H}_{2}$-dependent methanogenesis will then be thermodynamically unfavorable. The regulation of the ambient $\mathrm{H}_{2}$ by sulfate-reducing bacteria or methanogenic archaea at a minimum concentration constitutes a clear example of thermodynamic control which still enables these organisms to maintain the required energy yield of $\leq-10$ to $-20 \mathrm{~kJ}$ $\mathrm{mol}^{-1}$ (Hoehler et al., 2001). Acetate is another important and common substrate for sulfate reduction and methanogenesis in marine anoxic sediments. As compared to $\mathrm{H}_{2}$ concentrations, measured pore water concentrations of acetate in marine sediments have been reported to be 10 $\mu \mathrm{M}$ or more. This concentration is enough to support both sulfate reduction and methanogenesis 8almost all acetate in the sulfate zone is converted to $\mathrm{CO}_{2}$ instead of $\mathrm{CH}_{4}$ (King et al., 1983). This phenomenon has been explained by the ability of acetoclastic methanogens to grow in syntrophy with sulfate reducers which results in shifting from producing methane from acetate to feeding the sulfate reducers with $\mathrm{H}_{2}$ (Finke et al., 2007b). The competitive and potential syntrophic relationships between these two phylogenetic groups of microorganisms may therefore be more complex than has so far been recognized.

Similar to the fact that methanogens reside in the upper sulfate-rich sediments, recent data on the relative distribution of sulfate-reducing bacteria in Black Sea and Aarhus Bay sediments shows that SRB are present within the methane zone at similar high numbers as in the sulfate zone (Leloup et al., 2007, 2009). According to the study of Leloup et al. (2007), sulfate- and methanerich sediment layers showed the dominance of similar, novel cluster of $d s r A B$ sequences. $d s r A B$ are the functional marker genes encoding alpha- and beta-subunits of dissimilatory (bi)sulfite reductase that is used by sulfate-reducing microorganisms for energy conservation (Pester et al., 2012). Considering that the phylogenetic position of the novel $d s r A B$ cluster is in close vicinity of some sulfate-reducing microorganisms that have the ability to grow syntrophically (Leloup et al. 2007), it becomes obvious that the ability of sulfate reducers to perform both sulfidogenic and syntrophic lifestyle enables them to thrive in high- and low-sulfate environments in high numbers. On the other hand, Holmkvist et al. (2011) proposed that the sulfate reducers inhabiting the methane zone of Aarhus Bay live by reducing sulfate. They showed an immediate 10- to 40-fold increase in sulfate reduction after addition of both sulfate and organic substrates into the sediment and concluded that the low background sulfate concentration in the sediment is generated from 
the chemical reaction of downwards diffusing sulfide with deeply buried Fe(III) species and perhaps to a low diffusive flux of sulfate from above. Despite the occurrence of sulfate reduction in the methane zone, Holmkvist et al. (2011) also reported that sulfate reduction in the methane zone was only a fraction of ongoing methanogenesis and it corresponded to $0.1 \%$ of the total sulfate reduction in the sulfate zone. Therefore, it is relevant to understand how sulfate reducers interact with methanogenic communities when the sulfate is insufficient for complete oxidation of organic compounds.

\subsection{Outline of the thesis}

Sulfate reduction and methanogenesis are important terminal electron accepting processes in many anaerobic ecosystems including marine sediments, freshwater sediments, digesters, and propionate, butyrate, acetate, hydrogen and formate are the major end-products of fermentation during organic matter degradation in these ecosystems. Acetate, $\mathrm{H}_{2}$ and formate are key intermediates and serve as primary substrates for several terminal processes, such as sulfate reduction and methanogenesis. Sørensen et al. (1981) reported that acetate oxidation contributes for $40-50 \%$ to sulfate reduction in marine sediments while the contribution of $\mathrm{H}_{2}$ consumption is 5 - $10 \%$, propionate and butyrate oxidations are $10-20 \%$, in case of complete oxidation of propionate and butyrate. The conversion of propionate and butyrate is critical in deep sulfatedepleted sediments since they can be converted only by the combined action of several different physiological groups of bacteria and archaea. Despite the importance of these compounds in sulfate-rich and sulfate-depleted parts of marine sediments, it is often unclear what populations of microorganisms are involved in their degradation in the upper and lower parts of the sediment column. Therefore, we aimed to gain more insight into the propionate- and butyrate-degrading microbial communities residing at different depths of marine sediments and to better understand the factors affecting their growth, such as substrate and sulfate availability, and temperature. We also targeted to isolate butyrate and propionate-degrading syntrophic communities from different parts of the sediment. The sources of organic matter and the degradation of organic matter, the biogeochemical zones and the role of microorganisms in shaping these zones are discussed. The focus is brought on the butyrate and propionate conversions since they are considered as rate limiting steps in anaerobic degradation of organic matter.

Chapter 2 evaluates interspecies hydrogen transfer between and coexistence of marine methanogens and sulfate reducers using acetate as sole electron and carbon source in mixed pure 
cultures. To test interspecies hydrogen transfer, aceticlastic Methanosaeta (Methanotrix) concilii was cocultured with either hydrogenotrophic sulfate reducer, Desulfovibrio vulgaris, or hydrogenotrophic methanogen, Methanococcus maripaludis. Coexistence of $M$. concilii and Desulfobacter latus on acetate was investigated under sulfidogenic conditions in mixed pure cultures. The concentrations of substrates, intermediates, and electron acceptor were quantified by analytical methods at different time intervals. The total number of $16 \mathrm{~S}$ rRNA gene copies was determined regularly by quantitative PCR in order to define the growth curve of each microorganism during experiment. Gibbs free energies per reaction were calculated to determine the possibility of each reaction to occur under given culture conditions and with partner organism.

Chapter 3 aims to investigate butyrate-degrading sulfate-reducing and syntrophic communities in the sulfate, sulfate-methane transition and methane zone of Aarhus Bay, Denmark. In order to enrich for sulfate-reducing, syntrophic bacteria and methanogenic archaea, batch slurry incubations were prepared using the sediment retrieved from different zones. Some of these slurries were amended with $20 \mathrm{mM}$ sulfate to stimulate growth of sulfate-reducing communities. Some of the slurries were amended with $3 \mathrm{mM}$ sulfate to examine the growth behavior of sulfate-reducing and syntrophic communities, and some were incubated without sulfate to stimulate syntrophic communities. Two different temperatures were used for incubation; one is the in situ temperature and the other is a higher temperature to investigate its effect on the conversion rates and the possible change in ultimate community structure. The change in the bacterial and archaeal community structure was analyzed by DGGE using the genomic DNA sampled at different time points. The ultimate microbial community in each batch slurry was determined by pyrosequencing and Illumina MiSeq sequencing of the extracted DNA from the samples taken at the last incubation day.

Chapter 4 is similar to Chapter 3 and investigates the propionate-degrading sulfate-reducing and syntrophic communities inhabiting different biogeochemical zones of Aarhus Bay. The propionate degradation and the product formation was followed by regular gas and liquid sampling. The change in the bacterial and archaeal community structure was analyzed by DGGE using the genomic DNA sampled at different time points. The ultimate microbial community in each batch slurry was determined by pyrosequencing and Illumina MiSeq sequencing of the extracted DNA from the samples taken at the last incubation day. The investigations performed in Chapters 3 and 4 are intended to test the hypothesis that syntrophs are not easily out-competed by sulfate reducers, 
rather both physiological groups can coexist throughout the sediment at different sulfate concentrations. Furthermore, the demonstration of simultaneous sulfate reduction and methanogenesis during propionate and butyrate conversion throughout the sediment is important to understand the capabilities of the microbes involved.

Chapter 5 monitors intact polar membrane lipids (IPL) content of all the enrichment slurries studied in Chapters $3 \& 4$ and constitutes a complementary tool to the molecular analysis conducted in these chapters. IPL analysis was performed by high performance liquid chromatographyelectrospray ionization-mass spectrometry (HPLC/ESI/MS) on the original sediment samples and the enrichment slurry samples taken at the end of the incubation period. By comparing the IPL composition of both samples, we aimed to understand the factors shaping microbial community such as amended substrates, availability of sulfate, incubation temperature and the depth of the sediment.

Chapter 6 presents the further enrichment of propionate- and butyrate-degrading slurries to get more defined cultures and aims to take steps further for the isolation of new marine syntrophs. The enrichment slurries obtained in Chapters $3 \& 4$ were subcultured four times and the obtained microbial community was identified by cloning of bacterial and archaeal 16S rRNA gene analysis. The FISH method was used to visualize the syntrophic interactions and to capture the live microbes.

In Chapter 7, results obtained in this thesis are summarized and discussed with the knowledge presented in the literature. Additionally, future perspectives for research are presented. 



\section{Chapter 2}

\section{Methanogenic archaea and sulfate reducing bacteria co-cultured on acetate: teamwork or coexistence?}

This chapter has been published as:

Ozuolmez, D., Na, H., Lever, M.A., Kjeldsen, K.U., Jørgensen, B.B. and Plugge, C.M., (2015) Methanogenic archaea and sulfate reducing bacteria co-cultured on acetate: teamwork or coexistence? Frontiers in microbiology, 6(492): 1-12. 


\section{Abstract}

Acetate is a major product of fermentation processes and an important substrate for sulfate reducing bacteria and methanogenic archaea. Most studies on acetate catabolism by sulfate reducers and methanogens have used pure cultures. Less is known about acetate conversion by mixed pure cultures and the interactions between both groups. We tested interspecies hydrogen transfer and coexistence between marine methanogens and sulfate reducers using mixed pure cultures of two types of microorganisms. First, Desulfovibrio vulgaris subsp. vulgaris (DSM 1744), a hydrogenotrophic sulfate reducer, was cocultured together with the obligate aceticlastic methanogen Methanosaeta concilii using acetate as carbon and energy source. Next, Methanococcus maripaludis $\mathrm{S}_{2}$, an obligate $\mathrm{H}_{2}$ - and formate-utilizing methanogen, was used as a partner organism to $M$. concilii in the presence of acetate. Finally, we performed a coexistence experiment between $M$. concilii and an acetotrophic sulfate reducer Desulfobacter latus AcSR2. Our results showed that $D$. vulgaris was able to reduce sulfate and grow from hydrogen leaked by $M$. concilii. In the other coculture, M. maripaludis was sustained by hydrogen leaked by M. concilii as revealed by qPCR. The growth of the two aceticlastic microbes indicated co-existence rather than competition. Altogether, our results indicate that $\mathrm{H}_{2}$ leaking from $M$. concilii could be used by efficient $\mathrm{H}_{2}$-scavengers. This metabolic trait, revealed from coculture studies, brings new insight to the metabolic flexibility of methanogens and sulfate reducers residing in marine environments in response to changing environmental conditions and community compositions. Using dedicated physiological studies we were able to unravel the occurrence of less obvious interactions between marine methanogens and sulfate-reducing bacteria. 


\subsection{Introduction}

Marine coastal and shelf sediments are important sites for mineralization of organic matter deposited from land and from the marine photic zones (Jørgensen 1983). It is well established that under anoxic conditions, mineralization of complex organic matter requires cooperation between at least three trophic guilds (Schink and Stams, 2013). The first step in the degradation of organic matter is the hydrolysis of complex molecules into their oligomers or monomers. This step is followed by fermentation involving the degradation of these substrates to reduced organic compounds such as short chain fatty acids, and alcohols. In sulfate-rich sediments, sulfate-reducing bacteria (SRB) can use the products of primary fermentations and oxidize them to $\mathrm{CO}_{2}$. However, in sulfate-depleted methanogenic sediments, short chain fatty acids and alcohols are converted by secondary fermenters to acetate, formate, $\mathrm{H}_{2}$ and $\mathrm{CO}_{2}$, which are subsequently utilized by methanogenic archaea (MA) to produce $\mathrm{CH}_{4}$ (Muyzer and Stams, 2008; McInerney et al., 20o8; Stams and Plugge, 2009; Schink and Stams, 2013).

Acetate is a key intermediate in marine sediments as it is one of the major end-products of fermentation and serves as a primary substrate for several terminal electron accepting processes, like sulfate reduction and methanogenesis (Thamdrup et al. 2000; Sørensen et al., 1981; Christensen 1984; Parkes et al., 1989). There are two possible processes for methanogens to produce methane from acetate. In the first process acetate is cleaved to $\mathrm{CH}_{4}$ and $\mathrm{CO}_{2}$. This process is called aceticlastic methanogenesis and it is an energy-yielding reaction under standard conditions (Table 1, reaction 2). The second process, syntrophic acetate oxidation, was first proposed by Barker (Barker 1936), but attracted attention much later by Zinder and Koch (1984). Syntrophic acetate oxidation is a two-step process. It the first step, acetate is oxidized to $\mathrm{CO}_{2}$ by an aceticlastic microorganism with the generation of reducing equivalents, often as hydrogen. This step is endergonic and requires a hydrogenotrophic microorganism for the consumption of produced hydrogen (Table 1, reaction 1). In the second step, hydrogenotrophic methanogens scavenge that hydrogen and the overall reaction becomes thermodynamically favorable (Table 1 , the sum of reactions 1 and 5). Hydrogenotrophic sulfate reducers can also be involved in the second step and in case of SRB as the partner organism, the overall reaction is the same as if a sulfate reducer would oxidize acetate completely without a syntrophic partner (Table 1 , the sum of reactions 1 and 3 ). It has been shown in previous studies that not only aceticlastic bacteria but also aceticlastic methanogens can carry out the first step of syntrophic acetate oxidation (Phelps et al., 1985). In a syntrophic relationship, 
the chemical energy is shared via interspecies hydrogen transfer, so that not only sulfate reducers but also the aceticlastic methanogens would be able to grow in the sulfate zone of marine sediments. It is noteworthy that the energy yield from syntrophic acetate oxidation to sulfate is greater than the energy yield from aceticlastic methanogenesis (Table 1, the sum of reactions 1 and 3).

Interspecies $\mathrm{H}_{2}$ transfer has been studied using mixed pure cultures of the aceticlastic methanogen Methanosarcina barkeri and the hydrogenotrophic sulfate reducer Desulfovibrio vulgaris (Phelphs et al., 1985). Phelphs and colleagues co-cultivated M. barkeri with D. vulgaris and reported that $\mathrm{CO}_{2}$ production from acetate increased and $\mathrm{CH}_{4}$ production decreased in cocultures compared to pure cultures of M. barkeri, demonstrating interspecies hydrogen transfer. Syntrophic acetate oxidation by aceticlastic methanogens and hydrogenotrophic sulfate reducers was demonstrated for anoxic paddy soils (Achtnich et al. 1995) but has not been demonstrated for marine sediments so far.

Acetate concentrations in pore water of marine sediments are reported to be relatively high (typically $>10 \mu \mathrm{M}$ (Finke et al; 2007a)) and they are likely not under thermodynamic limitation in marine sediments, which makes acetate conversion by methanogens thermodynamically feasible even in the sulfate zone (Finke et al., 2007b). However, almost all acetate in the sulfate zone is converted to $\mathrm{CO}_{2}$, not to $\mathrm{CH}_{4}$ (Jørgensen and Parkes, 2010), suggesting the predominance of aceticlastic sulfate reduction. Thermodynamic mechanisms to explain the biogeochemical zonation in marine sediments in the presence of acetate are unclear. Finke and colleagues (2007b) suggested that acetate oxidation might proceed via interspecies $\mathrm{H}_{2}$ transfer. According to their hypothesis, aceticlastic methanogenesis is exergonic as long as acetate concentrations stay above $0.05 \mu \mathrm{M}$. Many studies have shown the existence of methanogens in sulfate-rich marine sediments (Wilms et al. 2007; Beck et al. 2011; Schippers et al. 2012). Methanosaeta sp. have been detected in marine sediments (Mori et al., 2012), with unknown identities, and the marine "Methanosaeta pelagica" has been recently isolated (Mori et al., 2012). Aceticlastic methanogens, specifically Methanosaeta species, may be important in contributing to acetate degradation in marine sediments, in particular the tidal flat sediments, which have an abundant supply of organic matter.

In this study, we investigated interspecies hydrogen transfer between aceticlastic Methanosaeta concilii and two hydrogenotrophic microorganisms, either a sulfate reducer, Desulfovibrio vulgaris, or a methanogen, Methanococcus maripaludis. We hypothesized that the existence of interspecies hydrogen transfer between aceticlastic methanogens and 
hydrogenotrophic sulfate reducers/methanogens in marine sediments would help to understand what controls the distribution of methanogens in sediments. Additionally, we tested coexistence between Methanosaetae concilii and Desulfobacter latus on acetate under sulfidogenic conditions in mixed pure cultures.

Table 1: Overview of reactions examined in this study. 1: Acetate oxidation, 2: Aceticlastic methanogenesis, 3: Hydrogenotrophic sulfate reduction, 4: Acetotrophic sulfate reduction, 5: Hydrogenotrophic methanogenesis, the sum of the reactions of 1 and 3 (reaction 4): Syntrophic acetate oxidation by an aceticlastic methanogen and a hydrogenotrophic sulfate-reducer, the sum of the reactions of 1 and 5 (reaction 2): Syntrophic acetate oxidation by an aceticlastic methanogen and a hydrogenotrophic methanogen. The calculations for standard conditions $(298 \mathrm{~K}, 1 \mathrm{~atm}, 1 \mathrm{M}$ reactants) were done with thermodynamic data from Lever (2012). The $\Delta \mathrm{G}_{\mathrm{r}}{ }^{\circ}$ values of the reactions shown in this table are different from the $\Delta \mathrm{G}_{\mathrm{r}}{ }^{\circ}$ values of the same reactions shown in other chapters. The reason is the difference in Gibbs free energies of formation $\left(\Delta \mathrm{Gf}^{\circ}\right)$ values for $\mathrm{H}_{2}$ and $\mathrm{CH}_{4}$ in aquous state (used in this chapter) and in the gaseous state (used in other chapters).

\begin{tabular}{|c|c|c|}
\hline Reaction number & Reactions & $\Delta \mathrm{G}_{\mathrm{r}}^{\circ}\left(\mathrm{kJ} \mathrm{mol}^{-1}\right)$ \\
\hline 1 & $\mathrm{CH}_{3} \mathrm{COO}^{-}+4 \mathrm{H}_{2} \mathrm{O} \rightarrow 4 \mathrm{H}_{2}+2 \mathrm{HCO}_{3}^{-}+\mathrm{H}^{+}$ & $214 \cdot 70$ \\
\hline 2 & $\mathrm{CH}_{3} \mathrm{COO}^{-}+\mathrm{H}_{2} \mathrm{O} \rightarrow \mathrm{CH}_{4}+\mathrm{HCO}_{3}^{-}$ & $-14 \cdot 74$ \\
\hline 3 & $4 \mathrm{H}_{2}+\mathrm{SO}_{4}^{2-}+\mathrm{H}^{+} \rightarrow \mathrm{HS}^{-}+4 \mathrm{H}_{2} \mathrm{O}$ & -262.06 \\
\hline 4 & $\mathrm{CH}_{3} \mathrm{COO}^{-}+\mathrm{SO}_{4}^{2-} \rightarrow \mathrm{HS}^{-}+2 \mathrm{HCO}_{3}^{-}$ & $-47 \cdot 36$ \\
\hline 5 & $\mathrm{HCO}_{3}^{-}+4 \mathrm{H}_{2}+\mathrm{H}^{+} \rightarrow \mathrm{CH}_{4}+3 \mathrm{H}_{2} \mathrm{O}$ & -229.44 \\
\hline
\end{tabular}

\subsection{Materials and methods}

\subsubsection{Strains and cultivation}

Methanosaeta concilii strain (DSM 2139) was adapted to $2 \% \mathrm{NaCl}$ conditions and maintained routinely on $10 \mathrm{mM}$ acetate. Desulfovibrio vulgaris subsp. vulgaris (DSM 1744), Desulfobacter latus AcRS2 (DSM 3381) and Methanococcus maripaludis S2 (DSM 14266) were obtained from the German 
Collection of Microorganisms and Cell Cultures (DSMZ, Braunschweig, Germany) and maintained routinely on $\mathrm{H}_{2} / \mathrm{CO}_{2}(80: 20 \%$, v/v) plus $10 \mathrm{mM}$ sulfate, $10 \mathrm{mM}$ acetate plus $10 \mathrm{mM}$ sulfate and $\mathrm{H}_{2} / \mathrm{CO}_{2}$ (80:20\%, v/v) respectively. All strains were grown in the same mineral salts medium (described below). Methanogenic archaea and sulfate-reducing bacteria were cultured routinely at $37^{\circ} \mathrm{C}$ and/or $30^{\circ} \mathrm{C}$, respectively.

\subsubsection{Media and growth conditions}

For the preparation of cocultures and maintaining the pure cultures, a marine, bicarbonatebuffered mineral salts medium was used. The anoxic medium contained the following components (grams/liter): $\mathrm{KH}_{2} \mathrm{PO}_{4}$ (o.41), $\mathrm{Na}_{2} \mathrm{HPO}_{4} \cdot 2 \mathrm{H}_{2} \mathrm{O}$ (o.53), $\mathrm{NH}_{4} \mathrm{Cl}$ (o.3), $\mathrm{NaCl}$ (o.3), $\mathrm{CaCl}_{2} \cdot 2 \mathrm{H}_{2} \mathrm{O}$ (o.11), $\mathrm{MgCl}_{2} \cdot 6 \mathrm{H}_{2} \mathrm{O}$ (o.1), $\mathrm{NaHCO}_{3}(4), \mathrm{Na}_{2} \mathrm{~S}_{9} \mathrm{H}_{2} \mathrm{O}$ (0.024) and $0.05 \%(\mathrm{w} / \mathrm{v}$ ) yeast extract (YE) (added only to the pure and cocultures of $D$. vulgaris strain). The medium was supplemented with $1 \mathrm{ml} /$ liter of acid and alkaline trace element solution (Stams et al., 1992). The medium was boiled and cooled to room temperature under an oxygen-free $\mathrm{N}_{2}$ flow. The medium was dispensed into $120 \mathrm{ml}$ serum bottles. The bottles were sealed with butyl rubber stoppers and crimp caps and the gas headspace was replaced with $1.7 \mathrm{~atm} . \mathrm{N}_{2} / \mathrm{CO}_{2}(80: 20 \% \mathrm{v} / \mathrm{v})$ and autoclaved.

Acetate from a concentrated sterile stock solution was added to the medium to a final concentration of $10 \mathrm{mM}$. Besides the substrate, vitamins (1 ml/liter) (Stams et al., 1992) were added from sterile stock solution to the medium. In order to reach the desired salt concentration ( $2 \%$, $\mathrm{w} / \mathrm{v}$ ), sterile anoxic artificial seawater, containing (in grams/liter) $\mathrm{NaCl}$ (40), $\mathrm{MgCl}_{2} \cdot 6 \mathrm{H}_{2} \mathrm{O}(10.8), \mathrm{KCl}$ (o.7), $\mathrm{CaCl}_{2} \cdot 2 \mathrm{H}_{2} \mathrm{O}$ (1) was added to serum bottles in same volume as the medium volume. The $\mathrm{pH}$ of the medium was set to 7 .

\subsubsection{Experimental design}

Microorganisms were cultivated in duplicate in $120 \mathrm{ml}$ serum vials with a final volume of $50 \mathrm{ml}$. Complete medium (30 ml) was inoculated with $20 \%(\mathrm{v} / \mathrm{v})$ of each microorganism to prepare cocultures. Final concentrations of acetate and sulfate in bacterial-archaeal cocultures were $10 \mathrm{mM}$, whereas archaeal-archaeal coculture contained only $10 \mathrm{mM}$ acetate. The flasks were flushed with $\mathrm{N}_{2} / \mathrm{CO}_{2}$ immediately after inoculation of each strain to remove residual $\mathrm{H}_{2}$ and $\mathrm{CH}_{4}$, leaving 1.7 bar of $\mathrm{N}_{2} / \mathrm{CO}_{2}(80: 20 \% \mathrm{v} / \mathrm{v})$ as the headspace. All inoculations were done aseptically and all cocultures were incubated under static conditions in the dark. Cocultures of methanogenic archaea were 
incubated at $37^{\circ} \mathrm{C}$ while bacterial-archaeal cocultures were incubated at $30^{\circ} \mathrm{C}$. Incubations lasted for 41 days for M. concilii-D. vulgaris and M. concilii-M. maripaludis cocultures and 21 days for $M$. concilii-D. latus cocultures. Gas and liquid samples were taken at different time intervals and analyzed for $\mathrm{H}_{2}, \mathrm{CH}_{4}$, acetate, sulfate, sulfide, dissolved inorganic carbon and biomass increase.

Pure cultures of respective microorganisms were cultivated in the presence of the required electron donor and acceptor as control. The culture conditions of pure cultures were explained in section 2.1. D. vulgaris was incubated at two different conditions in addition to its original culture condition; one was without $\mathrm{H}_{2} / \mathrm{CO}_{2}$ but with yeast extract addition $(0.05 \%, \mathrm{w} / \mathrm{v})$ and the other was without $\mathrm{H}_{2} / \mathrm{CO}_{2}$ but with YE $(0.05 \%, \mathrm{w} / \mathrm{v})$ and $10 \mathrm{mM}$ acetate. These controls were made to check for the ability of the strain to grow and reduce sulfate with YE and/or acetate in the absence of $\mathrm{H}_{2} / \mathrm{CO}_{2}$.

\subsubsection{Analytical methods}

$\mathrm{CH}_{4}$ was analyzed by gas chromatography with a Shimadzu GC-14B (Shimadzu, Kyoto, Japan) equipped with a packed column (Molsieve 13X, 60-80 mesh, $2 \mathrm{~m}$ length, $3 \mathrm{~mm}$ internal diameter) (Varian, Middelburg, The Netherlands) and a thermal conductivity detector set at $70 \mathrm{~mA}$. The injection volume was $0.2 \mathrm{ml}$. The oven temperature and the injector temperatures were both $100^{\circ} \mathrm{C}$. The detector temperature was $150^{\circ} \mathrm{C}$. Argon was the carrier gas at a flow rate of $30 \mathrm{ml} / \mathrm{min}$.

$\mathrm{H}_{2}$ was measured using a gas chromatograph equipped with pulsed discharge detector (PDD) (Trace Analytical, Bester, Amstelveen). The GC had Carboxen 1010 column, $3 \mathrm{~m}$ x $0.32 \mathrm{~mm}$ followed by a Molsieve $5 \mathrm{~A}$ column, $25 \mathrm{~m}$ x $0.32 \mathrm{~mm}$. The injection volume was $0.5 \mathrm{ml}$. The carrier gas was helium with a flow rate of $20 \mathrm{ml} / \mathrm{min}$. The column oven temperature was $90^{\circ} \mathrm{C}$, the injection oven temperature was $80^{\circ} \mathrm{C}$ and the detector temperature was $110^{\circ} \mathrm{C}$. The pressure was $200 \mathrm{kPa}$ and the input range was $64 \mathrm{nA}$.

Acetate from centrifuged (10,000 x $g$, $10 \mathrm{~min})$ samples of the culture media was analyzed by Thermo Scientific Spectrasystem HPLC system equipped with a Varian Metacarb $67 \mathrm{H} 300$ x $6.5 \mathrm{~mm}$ column kept at $30^{\circ} \mathrm{C}$ and running with $0.005 \mathrm{M}$ sulfuric acid as eluent. The eluent had a flow of 0.8 $\mathrm{ml} / \mathrm{min}$. The detector was a refractive index detector.

Sulfate concentrations were analyzed by Thermo Scientific Dionex HPLC equipped with an AS22 column (Thermo Scientific Dionex, Massachusetts, USA) with eluents of $0.235 \mathrm{~g} / \mathrm{l} \mathrm{NaHCO}_{3}$ and $2.576 \mathrm{~g} / \mathrm{l} \mathrm{Na}_{2} \mathrm{CO}_{3}$ at a flow rate of $1.2 \mathrm{ml} / \mathrm{min}$. The column temperature was $30^{\circ} \mathrm{C}$ and pressure was 130-16o bar. 
Sulfide measurements were done using the methylene blue method (Cline, 1969). Samples were diluted 1:1 with 5\% ZnAc solution directly after sampling, to precipitate all sulfide. The solution was stored at room temperature for at least 20 minutes in order to promote the precipitation of zinc sulfide. After color development, the concentration was measured on a MERCK Spectroquant ${ }^{\circledR}$ Multy at $670 \mathrm{~nm}$. Demi-water was used as a blank.

The $\mathrm{pH}$ was measured using Proline B210 $\mathrm{pH}$ electrode.

\subsubsection{DIC measurements}

For dissolved inorganic carbon (DIC) analysis, a $1 \mathrm{ml}$ glass vial containing glass beads was filled with culture sample till the liquid became convex on top and the vial was sealed with a screw cap. The vials were stored at $4^{\circ} \mathrm{C}$ until analysis. Total DIC concentrations were measured as gaseous $\mathrm{CO}_{2}$ after acidification of the liquid using a gas chromatograph (SRI 310C GC, SRI Instruments Europe $\mathrm{GmbH}$ ) equipped with a thermal conductivity detector (TCD).

\subsubsection{DNA extraction}

Biomass was harvested at selected time points by sampling $1 \mathrm{~mL}$ of culture after homogenization by vortexing, and centrifugation at 13,00o $\mathrm{g}$ for $20 \mathrm{~min}$. Genomic DNA was extracted from the pellet, using the PowerSoil ${ }^{\mathrm{R}}$ DNA Isolation kit (MoBio), following the manufacturer's instructions.

\subsubsection{Quantification of 16S rRNA genes by quantitative PCR}

The total number of 16S rRNA gene copies was quantified by SYBR Green assay, on the CFX96 TouchTM Real-time PCR Detection System (Bio-Rad). The primers used for amplifying bacterial 16S rRNA genes were Bac8F and Bac338Rabc (Juretschko et al. 1998; Daims et al. 1999). For Archaea, Arch8o6F and Arch958R were used (Takai and Horikoshi, 2000; DeLong, 1992). For the coculture of Methanosaeta concilii and Methanococcus maripaludis, Methanosaeta-specific primers (MSib 585F and Sae 835R; Conklin et al. 2006) and Methanococcales-specific primers (MCC495F and MCC832R; Yu et al. 2005) were used.

Prior to qPCR, the primers were tested by end-point PCR (annealing temperature gradient from $56^{\circ} \mathrm{C}$ to $65^{\circ} \mathrm{C}, 40$ PCR cycles) on DNA extracts from pure cultures of the respective strains to ensure the specificity of the qPCR assays. None of the primer pairs used showed any unspecific amplification of non-target groups. All primers are shown in Supplementary Table 1. 
Per PCR reaction, a total volume of $10 \mu \mathrm{L}$ mixture contained $5 \mu \mathrm{L}$ of iQ SYBR Green Supermix (Bio-Rad), $10 \mu \mathrm{M}$ of each primer and $1 \mu \mathrm{L}$ of $\sim 5 \mathrm{ng} / \mu$ l template DNA. The amplification program consisted of an initial activation step at $95^{\circ} \mathrm{C}$ for $3 \mathrm{~min}, 45$ cycles of: denaturation at $95{ }^{\circ} \mathrm{C}$ for $15 \mathrm{~s}$, annealing at $55^{\circ} \mathrm{C}$ for $30 \mathrm{~s}$ and elongation at $72^{\circ} \mathrm{C}$ for $30 \mathrm{~s}$, and a final extension step at $60^{\circ} \mathrm{C}$ for $31 \mathrm{~s}$. For reactions involving Methanosaeta- and Methanococcales-specific primer sets, the annealing temperature was adjusted to $60^{\circ} \mathrm{C}$. Melting curves were analyzed using the CFX ManagerTM software. The results were expressed as the number of cells per $\mu \mathrm{L}$ of sample, after calculating the number of $16 \mathrm{~S}$ rRNA genes per genome from reference strains with completely sequenced genomes, using Genbank (http://www.ncbi.nlm.nih.gov/genbank) and the RNAmmer 1.2 Server (http://www.cbs.dtu.dk/services/RNAmmer/) (Lagesen et al., 2007). The calculated number of 16S rRNA gene copies and the corresponding reference strains were: 2 for Methanosaeta concilii GP6, 5 for Desulfovibrio vulgaris subsp. vulgaris Hildenborough ${ }^{\mathrm{T}}, 4$ for Desulfobacter postgatei 2ac9, and 3 for Methanococcus maripaludis (strains $\mathrm{C}_{5}, \mathrm{C} 6, \mathrm{C}_{7}$, and $\mathrm{S}_{2}$ ).

\subsubsection{Calculation of Gibbs free energy $(\Delta G)$}

Gibbs free energies per reaction were calculated for the reactions shown in Table 1. For each reaction, the thermodynamic data for $\Delta \mathrm{G}_{\mathrm{f}}{ }^{\circ}, \Delta \mathrm{H}_{\mathrm{f}}{ }^{\circ}, \Delta \mathrm{V}_{\mathrm{f}}{ }^{\circ}$ (Table S2) were used to calculate $\Delta \mathrm{G}_{\mathrm{r}}{ }^{\circ}$ (standard Gibbs free energy of reaction), $\Delta \mathrm{H}_{\mathrm{r}}^{\circ}$ (standard enthalpy of reaction), $\Delta \mathrm{V}_{\mathrm{r}}^{\circ}$ (standard volume of reaction) (Table $S_{3}$ ) by subtracting the sum of products from the sum of reactants.

$\Delta G$ values of reactions are dependent on temperature, pressure and chemical concentrations. Thus, $\Delta \mathrm{G}_{\mathrm{r}}^{\circ}$ values were corrected taking into account the temperature, pressure and concentrations of reactants and products (Wang et al. 2010).

Standard Gibbs free energies of reactions were corrected for different temperatures using the integrated Gibbs-Helmholz equation:

$$
\Delta G^{\circ}(T)=T *\left(\frac{\Delta G^{\circ}}{298 K}+\left(\frac{\Delta H^{\circ}}{T}-\frac{\Delta H^{\circ}}{298 K}\right)\right)
$$

The effect of pressure on $\Delta \mathrm{G}^{\circ}$ value was calculated using the equation:

$$
\Delta G^{\circ}(P)=\Delta G^{\circ}(T)+\Delta V f^{\circ} * \frac{(P-1)}{9869}
$$


As last, Gibbs free energies per reaction were calculated using the measured products and reactants via the equation:

$$
\Delta \mathbf{G}=\Delta \mathbf{G}^{\circ}+R T \ln \mathbf{K}
$$

where $\Delta \mathrm{G}^{\circ}$ was calculated under standard conditions (Table $\mathrm{S}_{3}$ ), $R$ is the gas constant (o.0o8314 kJ $\left.\mathrm{mol}^{-1} \mathrm{~K}^{-1}\right)$, and $T$ is the absolute temperature $(298.15 \mathrm{~K})$. The activity coefficient values for bicarbonate and acetate (0.532), for water and $\mathrm{H}^{+}(1)$, for $\mathrm{H}_{2}$ and $\mathrm{CH}_{4}(1.24)$, for sulfate (0.104) and sulfide (o.41) were taken from Millero and Schreiber (1982) and Lever (2012).

\subsection{Results}

\subsubsection{M. concilii in coculture with $D$. vulgaris}

Methane formation started directly and increased with time (Figure $1 \mathrm{~A}$ ). $10 \mathrm{mM}$ acetate was fully converted into $\mathrm{CH}_{4}$. In 41 days, $0.9 \mathrm{mM}$ sulfate was consumed and sulfide accumulated to a concentration of $0.8 \mathrm{mM}$. The bulk of sulfate was reduced in the first 6 days where $\mathrm{H}_{2}$ concentration sharply decreased. After that point, there were only slight fluctuations in sulfate concentration. The $\mathrm{H}_{2}$ pressure in the cocultures was 3.5 Pa when measured on day 1, presumably as a result of carryover from the D. vulgaris inocula. Hydrogen levels sharply decreased to $1.08 \mathrm{~Pa}$ in a week and then slowly dropped to 0.83 Pa until day 20. Later on, it slowly increased and reached to 1.21 Pa by the last day of the experiment. Pure cultures of $M$. concilii had pressures of $1.03 \mathrm{~Pa} \mathrm{H}_{2}$ on average throughout the incubation period (Figure $\mathrm{S}_{1} \mathrm{~A}, \mathrm{~S}_{1} \mathrm{~B}$ ). $\mathrm{H}_{2}$ concentration in pure culture controls of $D$. vulgaris incubated with $\mathrm{YE}$ and acetate without $\mathrm{H}_{2}$ addition was 19.5 $\mathrm{Pa}$ and was $8 \mathrm{~Pa}$ when incubated with YE only (Figure S2A, S2B). The pressures dropped to $0.92 \mathrm{~Pa}$ in both controls by the end of $8^{\text {th }}$ day and remained constant during the rest of the incubation period. $\mathrm{H}_{2}$ concentration in control bottles did not change any further and same concentration was observed in cocultures. Thus $0.92 \mathrm{~Pa}_{2}$ (equivalent to $7.12 \mathrm{nM}$ ) was assumed to be the threshold $\mathrm{H}_{2}$ concentration for the $D$. vulgaris subsp. vulgaris strain. Gibbs free-energy changes in the coculture ranged between -36.2 and $-20.9 \mathrm{~kJ} / \mathrm{mol}$ for the conversion of acetate into methane and bicarbonate and between -168.6 and $-152.3 \mathrm{~kJ} / \mathrm{mol}$ $\mathrm{H}_{2}$ for hydrogenotrophic sulfate reduction (Figure $1 B$ ). These results showed that both reactions were favorable throughout the experiment. The most negative Gibbs free-energy values for both reactions were obtained in the beginning of experiment where acetate and hydrogen concentrations were at the highest levels. The highest Gibbs free-energy value for hydrogenotrophic 
sulfate reduction was $-152.3 \mathrm{~kJ} / \mathrm{mol}$, showing that the growth of $D$. vulgaris was thermodynamically feasible at the determined $\mathrm{H}_{2}$ concentrations.


C

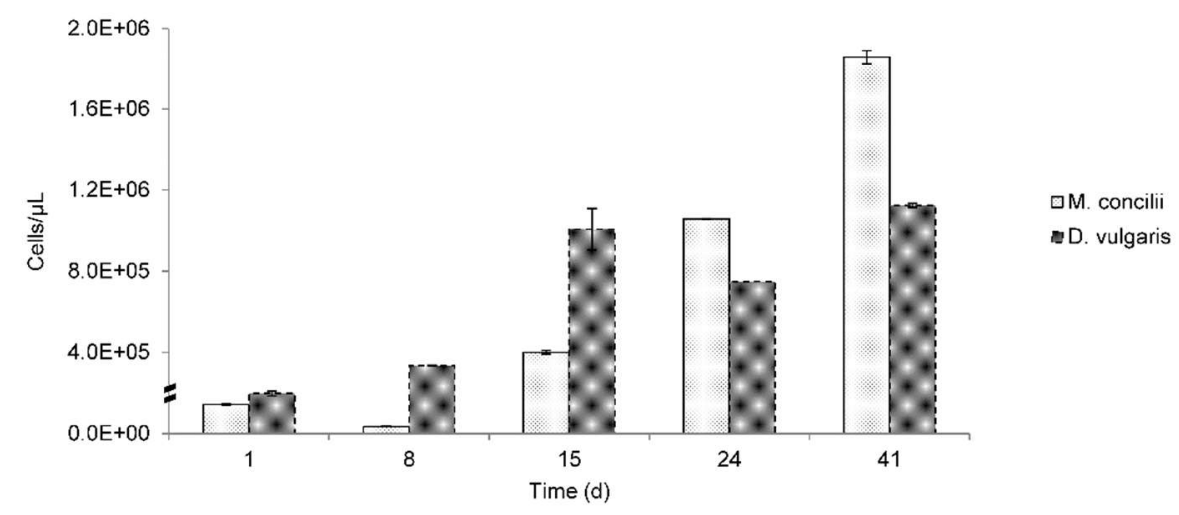

Figure 1. Growth on acetate by coculture of $M$. concilii and D. vulgaris subsp. vulgaris. (A) Changes in acetate, sulfate, sulfide, methane and hydrogen. (B) Actual Gibbs free-energy changes for acetate degradation to sulfide and bicarbonate and methane formation from acetate. (C) Growth quantified by qPCR in cells/ $\mu$ l. All data is average of 2 replicate incubations. 
qPCR results showed an increase in cell numbers of both organisms during the experiment (Figure $1 C$ ). The decrease in the cell numbers of $M$. concilii in the first 8 days coincided with a lag phase of acetate consumption. D. vulgaris cell numbers increased 2-fold in the same period with $\mathrm{H}_{2}$ consumption coupled to sulfate reduction. Between days 8 and 15, both M. concilii and D. vulgaris had the highest increase in their cell numbers with 11- and 3-fold increase, respectively. From day 15 until day 24, cell numbers of $M$. concilii increased 2.6-fold whereas $D$. vulgaris cell numbers decreased. In the last period of the incubation, both $M$. concilii and D. vulgaris showed growth with 1.8- and 1.5-fold increase in cell numbers, respectively. These results showed that $D$. vulgaris grew after consuming initial hydrogen to the threshold $\mathrm{H}_{2}$ value.

qPCR analysis of $D$. vulgaris pure culture controls showed growth during the experiment (Figure S3). D. vulgaris with YE and acetate showed the highest increase in cell numbers within the first 8 days. However, D. vulgaris in coculture grew to the highest cell density and showed a 5-fold increase in numbers after 15 days compared to day 1.

\subsubsection{M. concilii in coculture with M. maripaludis}

Acetate conversion started upon the start of the experiment. $\mathrm{CH}_{4}$ was produced from acetate and increased rapidly after 8 days of incubation (Figure $2 \mathrm{~A}$ ). As the first acetate addition was depleted by day 22, a second feed of acetate was given to the coculture. During the course of the experiment, $27 \mathrm{mM}$ acetate was consumed and $28 \mathrm{mM} \mathrm{CH}_{4}$ produced.

$\mathrm{H}_{2}$ level increased from 1.4 Pa to a peak concentration of 1.9 Pa during the first 15 days. This increase was concomitant to acetate consumption and $\mathrm{CH}_{4}$ production, which suggested $\mathrm{H}_{2}$ leakage from $M$. concilii cells during growth. After $\mathrm{H}_{2}$ reached the highest level, it was consumed by $M$. maripaludis to the lowest level which was 1.17 Pa. During the rest of the experiment, there were slight fluctuations in $\mathrm{H}_{2}$ level, apparent changes were not observed. In pure culture controls of $M$. concilii, average $\mathrm{H}_{2}$ levels were around 1.2 Pa and stayed constant throughout the experiment (Figure S1).

Gibbs free energies calculated for the conversion of acetate to methane and bicarbonate ranged between -36.2 and $-18.4 \mathrm{~kJ} / \mathrm{mol}$ and Gibbs free energies for hydrogenotrophic methanogenesis ranged between -12.7 and $-1.5 \mathrm{~kJ} / \mathrm{mol} \mathrm{H}_{2}$ (Figure $2 \mathrm{~B}$ ). $\Delta \mathrm{G}$ values showed that aceticlastic methanogenesis was favorable throughout the experiment. The Gibbs free energies for hydrogenotrophic methanogenesis were close to the biological energy quantum value. 


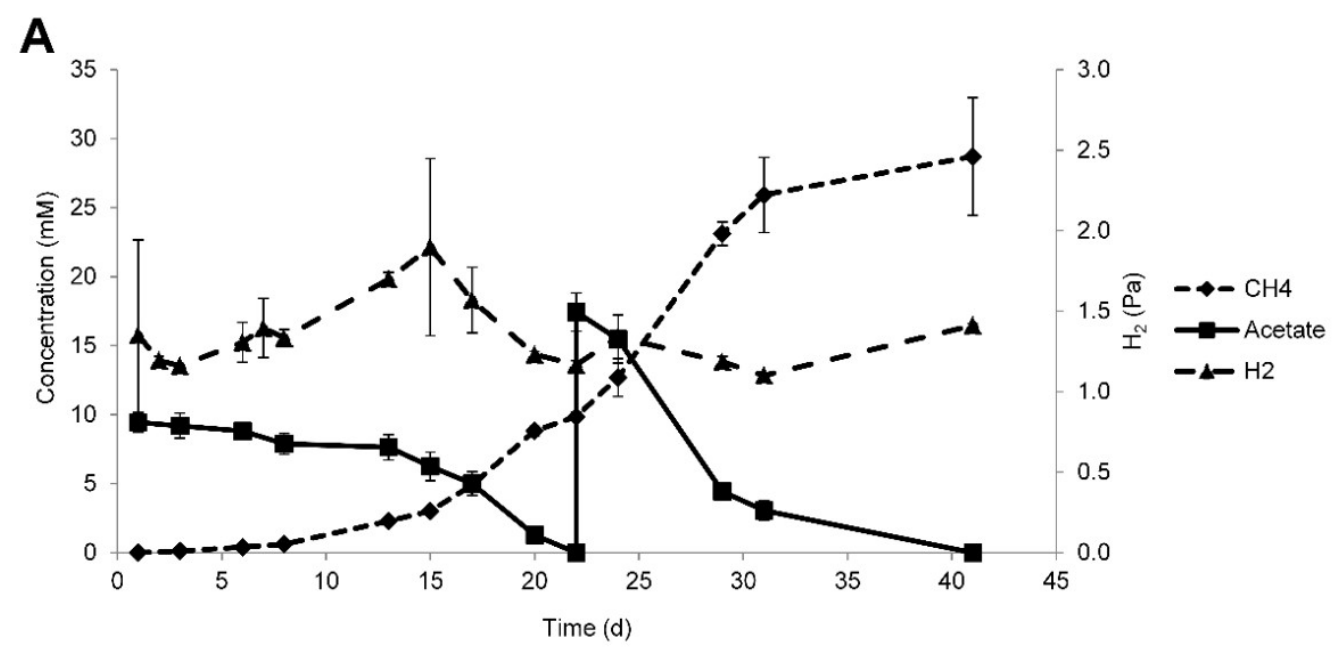

B
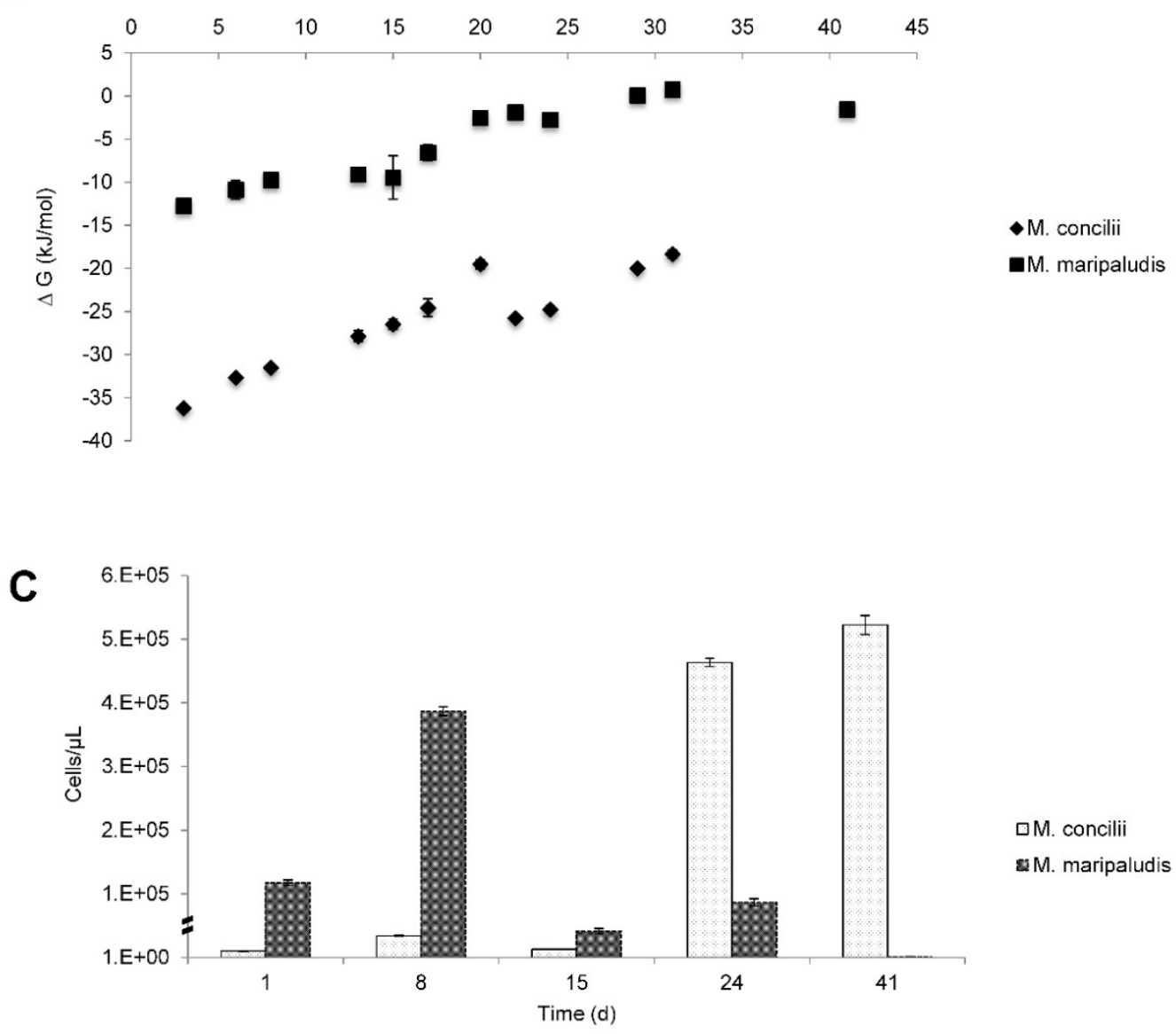

Figure 2. Growth on acetate by coculture of $M$. concilii and $M$. maripaludis. (A) Changes in acetate, methane and hydrogen. (B) Actual Gibbs free-energy changes for acetate degradation to methane formation from acetate. (C) Growth quantified by qPCR in cells/ $\mu$ l expressed. All data is average of 2 replicate incubations. 
According to the qPCR results, both organisms showed growth during the course of the study (Figure $2 \mathrm{C}$ ). As a result of acetate consumption starting in the beginning of the experiment, cell numbers of $M$. concilii increased 3-fold until 8 days. Similarly, M. maripaludis cell numbers increased 3-fold in the first week. A decline was detected in both M. concilii and M. maripaludis cell numbers between days 8 and 15, followed by an increase simultaneous to the consumption of acetate and hydrogen. Between days 15 and 24, M. concilii and M. maripaludis cell numbers increased 36- and 2-fold, respectively. After day 24, only 1-fold increase detected in $M$. concilii cell numbers whereas a decline in M. maripaludis cell numbers was observed.

\subsubsection{M. concilii in coculture with $D$. latus}

Acetate conversion coupled to sulfate reduction started by the initiation of the experiment while $\mathrm{CH}_{4}$ production from acetate conversion was observed after a 2 day lag period (Figure $\left.3 \mathrm{~A}\right)$. Both $M$. concilii and D. latus contributed to acetate conversion during the experiment. D. latus reduced 16 $\mathrm{mM}$ sulfate by the oxidation of acetate, whereas $M$. concilii contributed to the acetate oxidation by producing $1.4 \mathrm{mM} \mathrm{CH}_{4}$ on average in 21 days.

Under these conditions, Gibbs free energies ranged between -44 and $-54 \mathrm{~kJ} / \mathrm{mol}$ for the conversion of acetate into sulfide and bicarbonate and Gibbs free energies for acetate-driven methanogenesis ranged between -23 and $-35 \mathrm{~kJ} / \mathrm{mol}$ (Figure ${ }_{3} \mathrm{~B}$ ). $\Delta \mathrm{G}$ values showed that both reactions were favorable during the course of the experiment.

qPCR results indicate an increase in cell numbers of both organisms during the experiment (Figure ${ }_{3} C$ ). Between day 7 and 14, both M. concilii and D. latus increased their cell numbers 3.7and 2.4-fold, respectively. The highest cell increase was observed in the last week of the experiment. Increase in cell numbers of M. concilii was 36-fold whereas cell numbers of D. latus increased 14.6fold.

In an additional experiment where we used the same coculture combination, $\mathrm{CH}_{4}$ production started after few days of incubation when sulfate reduction was already ongoing (Figure $\mathrm{S}_{4}$ ). This coculture yielded $0.7 \mathrm{mM} \mathrm{CH}_{4}$ until all sulfate was reduced by D. latus, after which $M$. concilii consumed the rest of the acetate coupled to $\mathrm{CH}_{4}$ formation. After 53 days, $6 \mathrm{mM}$ acetate was consumed by $M$. concilii stoichiometrically, which was much slower than D. latus (37 days). 
A

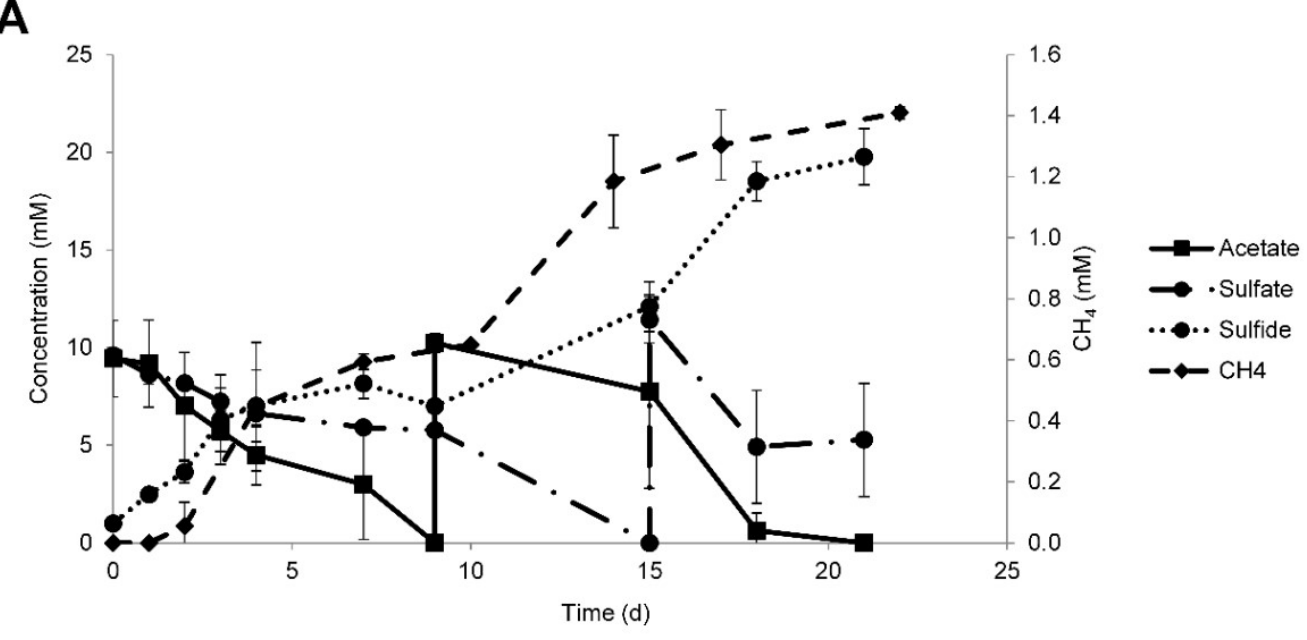

B

Time (d)
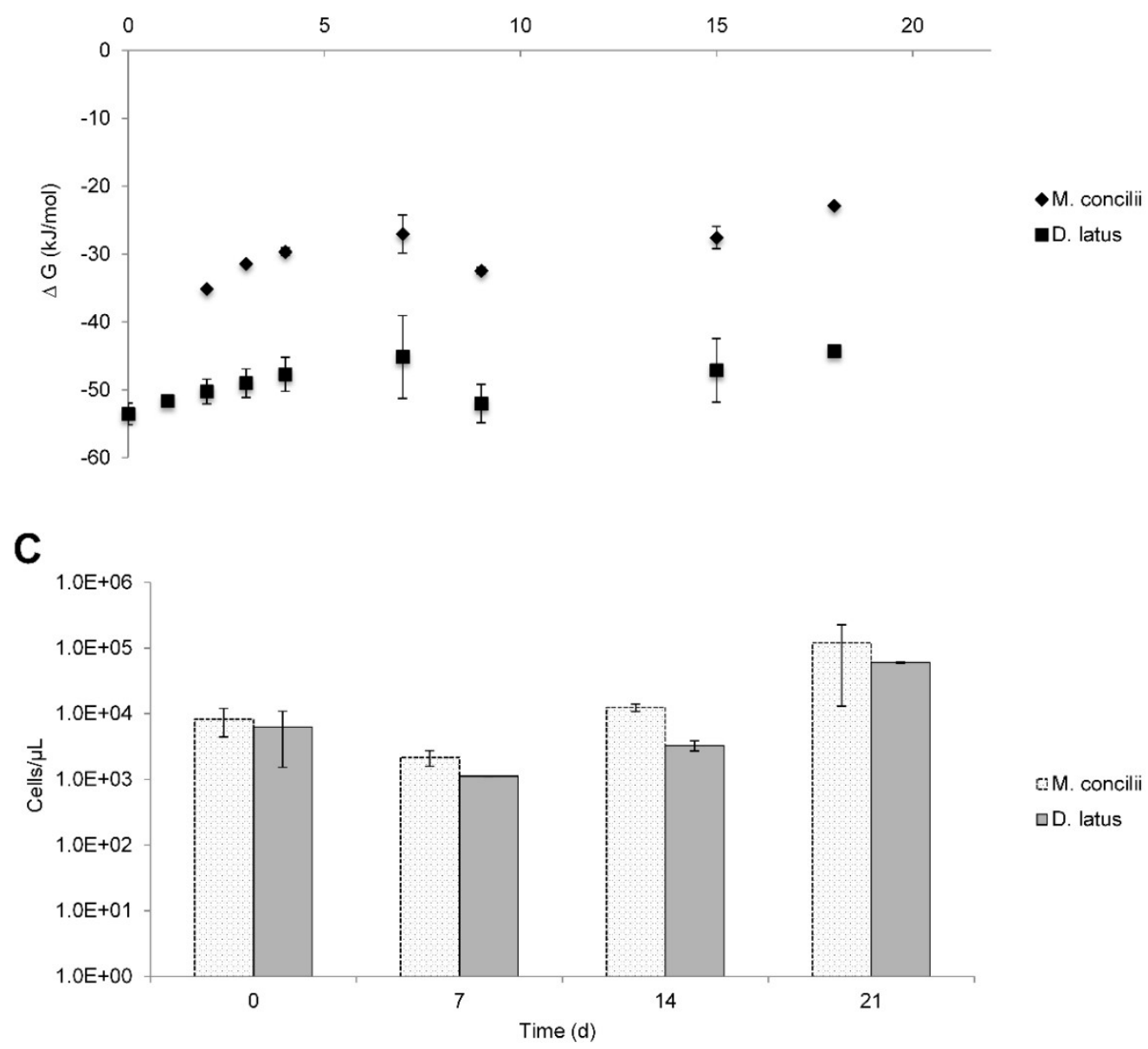

Figure 3. Growth on acetate by coculture of $M$. concilii and D. latus. (A) Changes in acetate, sulfate, sulfide and methane. (B) Actual Gibbs free-energy changes for acetate degradation to sulfide and bicarbonate and methane formation from acetate. (C) Growth quantified by qPCR in cells/ $\mu$ l. All data is average of 2 replicate incubations. 


\subsection{Discussion}

In this study, we tested interspecies hydrogen transfer in two different coculture combinations. We cocultured an obligate aceticlastic methanogen, Methanosaeta concilii together with a hydrogenotrophic sulfate reducer, Desulfovibrio vulgaris or a hydrogenotrophic methanogen Methanococcus maripaludis. We aimed to investigate whether hydrogen leakage from Methanosaeta is possible under conditions where the hydrogen is efficiently scavenged by hydrogenotrophic sulfate reducers or methanogens and whether such a hydrogen leakage enables the growth of the consuming organisms. Additionally, we tested coexistence between Methanosaetae concilii and Desulfobacter latus on acetate under sulfidogenic conditions in mixed pure cultures.

\subsubsection{M. concilii in coculture with $D$. vulgaris or $M$. maripaludis}

In the cocultures of $M$. concilii and D. vulgaris, acetate was converted into $\mathrm{CH}_{4}$ and $\mathrm{CO}_{2}$ in $1: 1$ stoichiometry during the incubation period. In case of syntrophic acetate oxidation by an aceticlastic methanogen and a hydrogenotrophic sulfate reducer couple, the expected overall reaction is exactly the same as if the sulfate reducer oxidized acetate completely without a syntrophic partner (Table 1, reaction 4). Taking this into account, our data on the stoichiometry of the reaction do not point directly toward such a relationship.

Sulfate reduction occurred especially in the beginning of the experiment coupled to the oxidation of residual hydrogen from the inoculum. As a result of sulfate reduction, sulfide production occurred within the same time period. A minor discrepancy between sulfide produced and sulfate consumed may be attributed to chemical oxidation of $\mathrm{HS}^{-}$to polysulfide by trace levels of oxygen.

$\mathrm{H}_{2}$ measurements were of critical importance in our study to evaluate whether Methanosaeta was leaking hydrogen in coculture with a hydrogenotrophic partner. Results showed that $D$. vulgaris could couple hydrogen consumption to sulfate reduction in the first 8 days of the experiment and brought hydrogen levels to threshold concentrations and hydrogen concentrations remained at a constant low level similar to the level observed in M. concilii mono cultures (Fig. S1). Many $\mathrm{H}_{2}$ measurement studies were performed in different ecosystems and in pure cultures to determine threshold $\mathrm{H}_{2}$ concentrations for different terminal electron accepting reactions. (Lovley, 1985; Cord-Ruwisch et al., 1988; Lovley and Godwin, 1988; Conrad, 1996; Hoehler et al., 1998). 
According to these studies, threshold $\mathrm{H}_{2}$ concentrations for sulfate reduction were found in range between 5-95 $\mathrm{nM}$. Our results show an average of $7 \mathrm{nM}$ hydrogen in mono- and cocultures, which was in line with these observations. Taking into account that different threshold concentrations exist for growth and substrate degradation, D. vulgaris could benefit from traces of $\mathrm{H}_{2}$ leaked by $M$. concilii and coupled this to its growth. The calculated Gibbs free energy values show that the hydrogenotrophic sulfate reduction reaction was thermodynamically feasible with the hydrogen concentrations in the cocultures throughout the study (Fig. 1B). Apparently, D. vulgaris was extremely efficient, and needed only a very little amount of hydrogen to produce sufficient energy for growth (Fig. $1 \mathrm{C}$ ). Moreover, comparing pure culture with the coculture, hydrogen levels in Methaosaeta suggested that cocultivation can deviate electrons towards hydrogen production (Fig. 1B, Fig. S1).

Thus, this result supports our hypothesis that a minor part of the acetate was converted via the production of hydrogen.

In the other coculture combination, we used Methanococcus maripaludis, a methanogen that can use formate and/or $\mathrm{H}_{2} / \mathrm{CO}_{2}$ as carbon and energy source (Jones et al., 1983), as partner organism with $M$. concilii. In the presence of the methanogen as partner organism in syntrophic acetate oxidation, the net reaction is exactly the same as if acetate was cleaved by an aceticlastic methanogen (Table 1, reaction 2). In our study, the overall stoichiometry of the reaction, with slightly higher methane production, fits with both possibilities of acetate oxidation.

The trend in hydrogen concentration was different from that the trend in hydrogen concentration in the $M$. concilii and D. vulgaris coculture. The initial hydrogen concentration in the coculture was lower and an increase in hydrogen production was observed between day 3 and day 15. This increase was concomitant to acetate consumption and $\mathrm{CH}_{4}$ production, which suggests $\mathrm{H}_{2}$ leakage from $M$. concilii cells during growth. In the $M$. concilii control monoculture at $37^{\circ} \mathrm{C}$, there was no evidence for $\mathrm{H}_{2}$ accumulation as $\mathrm{H}_{2}$ level remained constant around $1.2 \mathrm{~Pa}$ throughout the experiment (Fig. S1). Therefore we speculated that $M$. maripaludis induced divergence of electrons from $M$. concilii and scavenged hydrogen leaked by $M$. concilii.

Comparing both cocultures, the $\mathrm{H}_{2}$ concentration in M. concilii-M. maripaludis coculture was higher than in $M$. concilii-D. vulgaris coculture, which can be attributed to the ability of $D$. vulgaris to reduce $\mathrm{H}_{2}$ concentrations to lower levels than M. maripaludis. Our data on threshold $\mathrm{H}_{2}$ concentrations determined for M. maripaludis ( $10 \mathrm{nM})$ fit with the finding of Hoehler and 
colleagues (1998) where threshold $\mathrm{H}_{2}$ concentrations for methanogens were reported to be around $13 \mathrm{nM}$.

$\Delta \mathrm{G}$ values showed that aceticlastic methanogenesis was favorable throughout the experiment. On the other hand, $\Delta \mathrm{G}$ values for hydrogenotrophic methanogenesis were close to the minimum biological energy quantum that permit organisms to grow (Hoehler et al., 2001). We used batch cultures to demonstrate the growth of both organisms. However accumulating methane in the bottles had a negative effect on the overall Gibbs free energy. If we calculate the Gibbs free energy using $1 \mathrm{mM}$ of methane, a value that is more realistic in marine sediments, the energy ranges from -7 to $-14 \mathrm{~kJ} / \mathrm{mol}$. Likewise, it was reported that methanogen yields may be -10 to $-15 \mathrm{~kJ} / \mathrm{mol}$ in marine sediments (Hoehler et al., 2001; Finke et al., 2007b; Jørgensen and Parkes, 2010). The decline in $M$. marilaudis cell numbers after day 24 can be explained by the decay rates of $M$. maripaludis. It is known that hydrogenotrophic methanogens have a high decay rate when left without substrate and stabilized in iron sulfide precipitates (Stams et al., 1992).

Taken together, we can speculate that the hydrogenotrophic methanogen benefited from the hydrogen leaked during the growth of the aceticlastic methanogen. Our findings on growth trend, $\Delta \mathrm{G}$ values and aforementioned reference studies showed the capability of M. maripaludis to metabolize and grow on $\mathrm{H}_{2}$ leaked by $M$. concilii. In this context it could be speculated that the hydrogen scavengers may act as parasites, as they benefit from the leakage of hydrogen by Methanosaeta.

There are several studies that demonstrated interspecies hydrogen transfer in defined cocultures (McInerney and Bryant, 1981; Phelps et al., 1985; de Bok et al., 2002b). In one of those studies, mixed pure cultures of Methanosarcina barkeri and Desulfovibrio vulgaris were tested for interspecies hydrogen transfer under high sulfate conditions using methanol and acetate as carbon and energy sources (Phelps et al., 1985). It is known that M. barkeri can produce trace amounts of $\mathrm{H}_{2}$ during growth on acetate in pure culture and use some of the substrate for growth (Phelps et al., 1985; Valentine et al., 200o). They reported decreased $\mathrm{CH}_{4}$ production and doubled $\mathrm{CO}_{2}$ formation when acetate was oxidized in coculture. Lower hydrogen concentrations were measured in coculture compared to the pure cultures of the methanogen, meaning that $D$. vulgaris consumed hydrogen produced by $M$. barkeri. The authors claimed that D. vulgaris caused a decrease in methanogenesis by means of linking interspecies hydrogen transfer to sulfate reduction.

Methanosarcina species are known to be generalists, they have low affinity for acetate and have a minimum threshold for acetate of around o.2 - $1.2 \mathrm{mM}$ (Jetten et al., 1992). On the other 
hand, Methanosaeta species are specialists, they consume only acetate as carbon and energy source and their minimum threshold for acetate is $7-70 \mu \mathrm{M}$ (Jetten et al., 1992). As acetate concentrations in the pore water of marine sediments are usually less than $20 \mu \mathrm{M}$ (between 8-45 $\mu \mathrm{M}$ ) (Christensen and Blackburn 1982; Wellsbury and Parkes 1995; Finke et al. 2007a), conditions appear to be suitable for Methanosaeta rather than for Methanosarcina. Many clones closely related to Methanosaeta have been detected in marine sediments (Mori et al., 2012), with unknown identities, however Methanosaeta pelagica has been recently isolated (Mori et al., 2012). Undoubtedly, Methanosaeta is one of the most recalcitrant methanogens and is difficult to enrich and isolate primarily because of slow growth. Hydrogen production from Methanosaeta was demonstrated for Methanosaeta thermophila when growing on acetate (Valentine et al., 200o), and here we reported for the first time hydrogen leakage from a mesophilic halotolerant Methanosaeta.

\subsubsection{M. concilii in coculture with $D$. latus}

M. concilii and D. latus grew well in coculture (Figure 3). Methane production occurred even in the presence of high sulfate concentrations $(7 \mathrm{mM})$. In the presence of non-limiting acetate concentrations, there was only minor competition for acetate between $M$. concilii and $D$. latus, as it was indicated by the concomitant sulfate reduction and methane production starting from the beginning of the experiment. qPCR data showed that $M$. concilii had an efficient biomass production at the end of the experiment. Additional data showed the same results, with slow, but steady production of methane after depletion of sulfate (Figure $\mathrm{S}_{4}$ ). Taken together, it is obvious that acetate conversion by aceticlastic methanogens in the presence of high sulfate and active aceticlastic sulfate reducers is possible. The concept of SRB and methanogen predominance in highsulfate and low-sulfate environments, respectively, was established through the accumulation of results from a vast number of studies since 1980s (Ward and Winfrey, 1985; Widdel, 1988). Later, the coexistence of methanogens and SRB was observed in the presence of non-limiting sulfate concentrations in different environments (Dar et al., 2008). Coexistence of SRB and MA has been determined in organic-rich sediments with methane production rates accounting for $<10 \%$ of the sulfate reduction rates (Crill and Martens, 1986). This provides a possible explanation for the coexistence of SRB and MA in this sulfate-rich medium as the concentration of acetate either exceeds the competition level or it is used noncompetitively. 


\subsubsection{New insights in metabolic flexibility}

Interspecies hydrogen transfer has been studied in different anoxic environments (e.g., freshwater and marine sediments, flooded soil, landfills and sewage digesters) for long time and its importance and mechanism in complete mineralization of organic matter has been well documented (McInerney et al., 2008; Stams and Plugge, 2009). Moreover, interspecies formate transfer has been put forward as an alternative way of syntrophy and equally important for electron transfer between microorganisms (Boone et al., 1989; de Bok et al., 2002b; de Bok et al., 2004). Recent studies have described a new concept, direct interspecies electron transfer (DIET), where two Geobacter species form large, electrically conductive aggregates and establish electrical connections via the pili of both species to transfer electrons (Summers et al., 2010). In addition, DIET has been reported to occur in coculture of aceticlastic Methanosaeta harundinacea and exoelectrogen Geobacter metallireducens. In this coculture, $M$. harundinacea was found to convert acetate produced from ethanol metabolism and accept additional electrons via DIET for the reduction of carbon dioxide to methane; thus ethanol was converted to methane stoichiometrically (Rotaru et al., 2014). The authors have reported that transcript abundance of the genes for the enzymes necessary for the reduction of carbon to methane was high in the aggregates (Rotaru et al., 2014). Similar findings were reported previously in comparative genome analysis study of Methanosarcina mazei and Methanosaeta thermophila (Smith and Ingram-Smith, 2007). In this study, it was shown that the two genera use different enzymes to catalyze the first step of aceticlastic methanogenesis, but the majority of the core steps of the pathway were similar, except for the differences in electron transfer and energy conservation. Additionally, they identified the genes required for enzymes to catalyze $\mathrm{CO}_{2}$ reduction to $\mathrm{CH}_{4}$ in Methanosaeta thermophila genome (Smith and Ingram-Smith, 2007).

Given that Methanosaeta genus members are unable to use hydrogen directly to reduce $\mathrm{CO}_{2}$, these findings become important to exhibit different metabolic capabilities of Methanosaeta species to survive under hydrogen and acetate deficient conditions and thrive in methanogenic environments. In another recent study, it was found that both wild type and hydrogenase-deletion mutant of Methanococcus maripaludis could produce methane by uptake of cathodic electrons from a graphite electrode, which serves another model to direct electron uptake by methanogens (Lohner et al., 2014). These newly proposed properties of Methanosaeta and Methanoccocus indicate a variety of mechanisms for microbial electron uptake, and suggest that these methanogens may thrive in marine sediments in close contact with each other for the ultimate metabolism of substrates and that they are capable of responding to changes in environmental 
conditions. Future experiments on environments with fluctuating sulfate levels could apply individual based technologies to reveal the in situ metabolism of the microorganisms present.

\subsection{Conclusions}

In conclusion, we show that an obligate aceticlastic methanogen, Methanosaeta concilii, leaked sufficient hydrogen to support the growth of a hydrogenotrophic sulfate reducer, $D$. vulgaris, or a hydrogenotrophic methanogen, M. maripaludis, when cultured together. The other important outcome of this study was the coexistence of the aceticlastic methanogen and an aceticlastic sulfate reducer in the presence of high sulfate concentration. These results bring more insights into the metabolic flexibility of methanogens and sulfate reducers residing in marine environments to adapt to changing environmental conditions and community.

\section{Acknowledgements}

We thank Prof. Dr. Fons Stams for helpful discussions, Ton van Gelder and Karina Bomholt Henriksen for technical assistance and Jan Gerritse (Deltares, The Netherlands) for his help with the hydrogen measurements. This work has been funded by the Wimek Graduate School of Wageningen University, Darwin Center for Biogeosciences (the Netherlands), the Danish National Research Foundation and the Max Planck Society (Germany). We also thank the funding support from the European Research Council (ERC) Advanced Grant 'Microbial life under extreme energy limitation (MICROENERGY)' awarded to Bo Barker Jørgensen under the European Union's Seventh Framework Program. 


\section{Supplementary data}

Table S1. PCR primers used in the study.

\begin{tabular}{ccc}
\hline Primer Name & Sequence (5' $\mathbf{- 3}$ ') & Reference \\
\hline Bac8F & AGAGTTTGATYMTGGCTCAG & Juretschko et al., 1998 \\
Bac338Rabc & GCWGCCWCCCGTAGGWGT & Daims et al., 1999 \\
Arch806F & ATTAGATACCCSBGTAGTCC & Takai \& Horikoshi, 2000 \\
Arch958R & YCCGGCGTTGAMTCCAATT & DeLong, 1992 \\
MS1b 585F & CCGGCCGGATAAGTCTCTT GA & Conklin et al., 2006 \\
Sae 835R & GACAACGGTCGCACCGTGGCC & Conklin et al., 2006 \\
MCC495F & TAAGG GCTGG GCAAGT & Yu et al., 2005 \\
MCC832R & CACCT AGTTC GCAGAGTTTA & Yu et al., 2005 \\
\hline
\end{tabular}

Table S2. Thermodynamic data of aqueous educts and products under standard conditions.

\begin{tabular}{|c|c|c|c|c|}
\hline Compound & $\Delta G_{f}^{\circ}\left(\mathrm{kJ} \mathrm{mol}^{-1}\right)$ & $\Delta H_{\mathrm{f}}^{\circ}\left(\mathrm{kJ} \mathrm{mol}^{-1}\right)$ & $\Delta V_{\mathrm{f}}{ }^{\circ}\left(\mathrm{cm}^{3} \mathrm{~mol}^{-1}\right)$ & Reference \\
\hline $\mathrm{CH}_{3} \mathrm{COO}^{-}$ & -369.4 & -486.4 & 40.5 & Shock and Helgeson (1990) \\
\hline $\mathrm{HCO}_{3}{ }^{-}$ & -586.9 & -692.0 & 24.6 & $\begin{array}{l}\text { Wagman et al. (1982), } \\
\text { Shock et al. (1997) }\end{array}$ \\
\hline $\mathrm{H}_{2} \mathrm{O}$ & -237.18 & -285.83 & 18.02 & Amend and Shock 2001 \\
\hline $\mathrm{H}^{+}$ & 0.0 & 0.0 & 0.0 & Shock et al. (1997) \\
\hline $\mathrm{H}_{2}$ & 17.6 & -4.2 & 25.2 & $\begin{array}{l}\text { Wagman et al. (1982), } \\
\text { Shock and Helgeson (1990) }\end{array}$ \\
\hline $\mathrm{CH}_{4}$ & -34.47 & -87.96 & 37.3 & Shock and Helgeson (1990) \\
\hline $\mathrm{SO}_{4}^{2-}$ & -744.96 & -910.21 & 13.88 & Shock et al. (1997) \\
\hline $\mathrm{HS}^{-}$ & 11.97 & -16.12 & 20.65 & Shock et al. (1997) \\
\hline
\end{tabular}

Table S3. Standard free energy of reaction $\left(\Delta G \mathrm{r}^{\circ}\right)$, standard enthalpy of reaction $\left(\Delta \mathrm{Hr}^{\circ}\right)$ and standard volume of reaction $\left(\Delta V \mathrm{r}^{\circ}\right)$ data of aqueous educts and products under standard conditions.

\begin{tabular}{|c|c|c|c|}
\hline Reactions & $\Delta \mathrm{Gr}^{\circ}\left(\mathrm{kJ} \mathrm{mol}^{-1}\right)$ & $\Delta \mathrm{Hr}^{\circ}\left(\mathrm{kJ} \mathrm{mol}^{-1}\right)$ & $\Delta \mathrm{Vr}^{\circ}\left(\mathrm{mol}^{-1}\right)$ \\
\hline $\mathrm{CH}_{3} \mathrm{COO}^{-}+4 \mathrm{H}_{2} \mathrm{O} \rightarrow 4 \mathrm{H}_{2}+2 \mathrm{HCO}_{3}^{-}+\mathrm{H}^{+}$ & 214.70 & 229.12 & 37.42 \\
\hline $\mathrm{CH}_{3} \mathrm{COO}^{-}+\mathrm{H}_{2} \mathrm{O} \rightarrow \mathrm{CH}_{4}+\mathrm{HCO}_{3}^{-}$ & -14.74 & -7.70 & 3.38 \\
\hline $4 \mathrm{H}_{2}+\mathrm{SO}_{4}{ }^{2-}+\mathrm{H}^{+} \rightarrow \mathrm{HS}^{-}+4 \mathrm{H}_{2} \mathrm{O}$ & -262.06 & -232.59 & -21.95 \\
\hline $\mathrm{CH}_{3} \mathrm{COO}^{-}+\mathrm{SO}_{4}^{2-} \rightarrow \mathrm{HS}^{-}+2 \mathrm{HCO}_{3}^{-}$ & -47.36 & -3.47 & 15.47 \\
\hline $\mathrm{HCO}_{3}{ }^{-}+4 \mathrm{H}_{2}+\mathrm{H}^{+} \rightarrow \mathrm{CH}_{4}+3 \mathrm{H}_{2} \mathrm{O}$ & -229.44 & -236.82 & -34.04 \\
\hline
\end{tabular}


Figure S1. Monocultures of Methanosaeta concilii at $30^{\circ} \mathrm{C}(\mathrm{A})$ and $37^{\circ} \mathrm{C}(\mathrm{B})$.
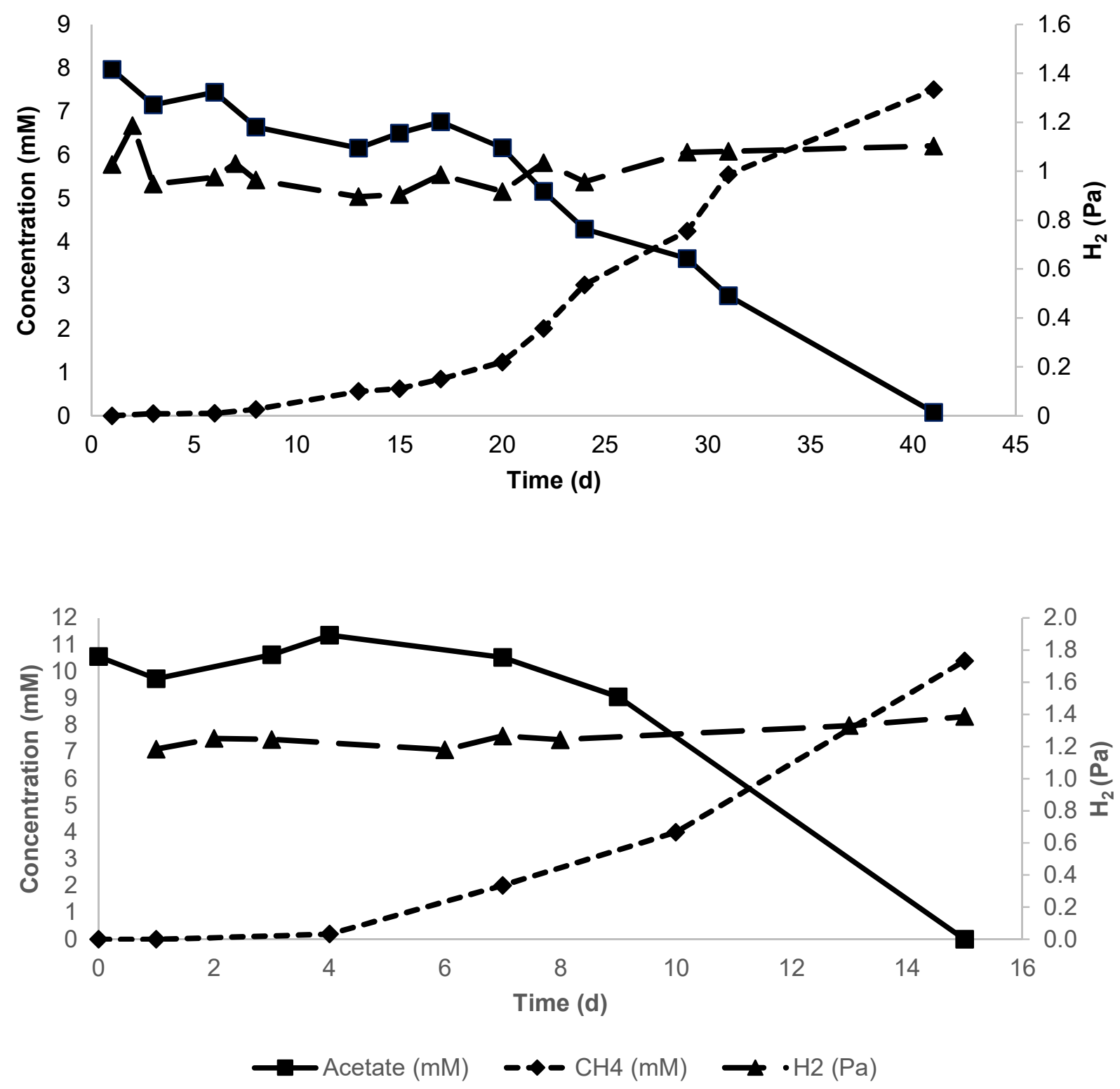
Figure S2. Monocultures of Desulfovibrio vulgaris without addition of $\mathrm{H}_{2} / \mathrm{CO}_{2}$, with yeast extract only (A), with yeast extract and acetate (B).
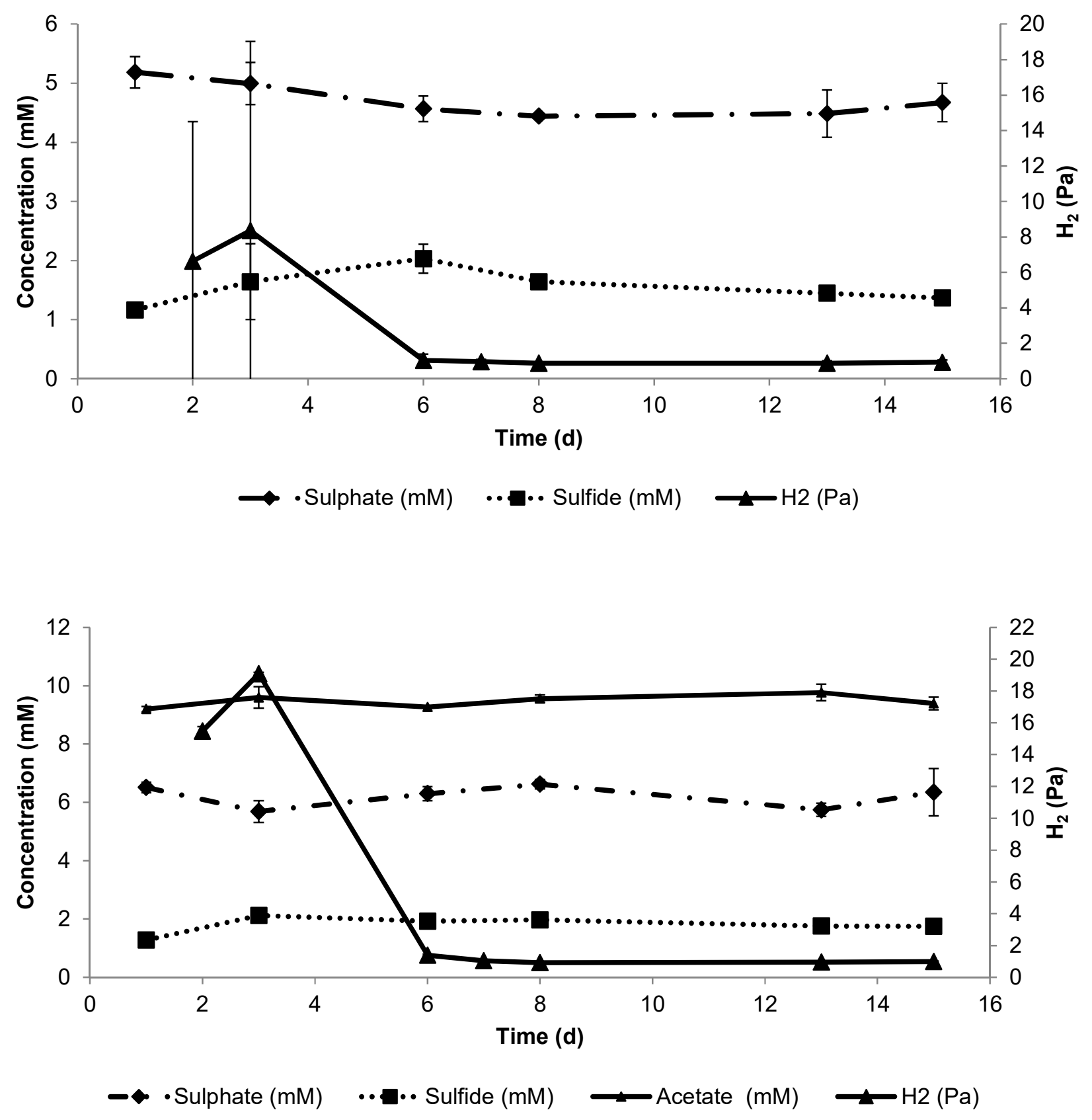
Figure S3. Growth in monocultures of $D$. vulgaris without addition of $\mathrm{H}_{2} / \mathrm{CO}_{2}$ but with yeast extract, without $\mathrm{H}_{2} / \mathrm{CO}_{2}$, but with yeast extract plus acetate and D. vulgaris in coculture, quantified by qPCR.

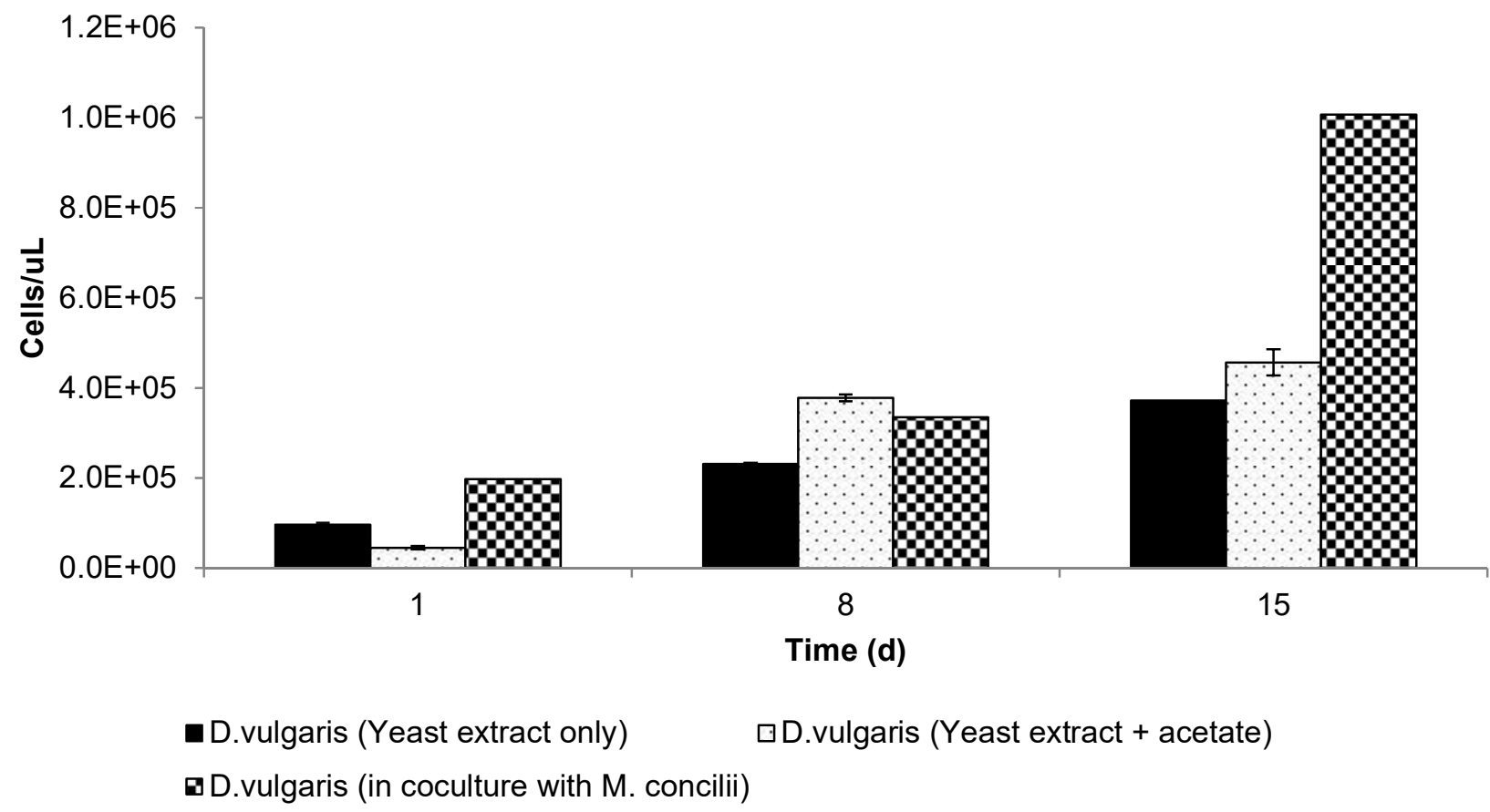

Figure S3. Growth of Methanosaeta concilii and Desulfobacter latus on acetate.

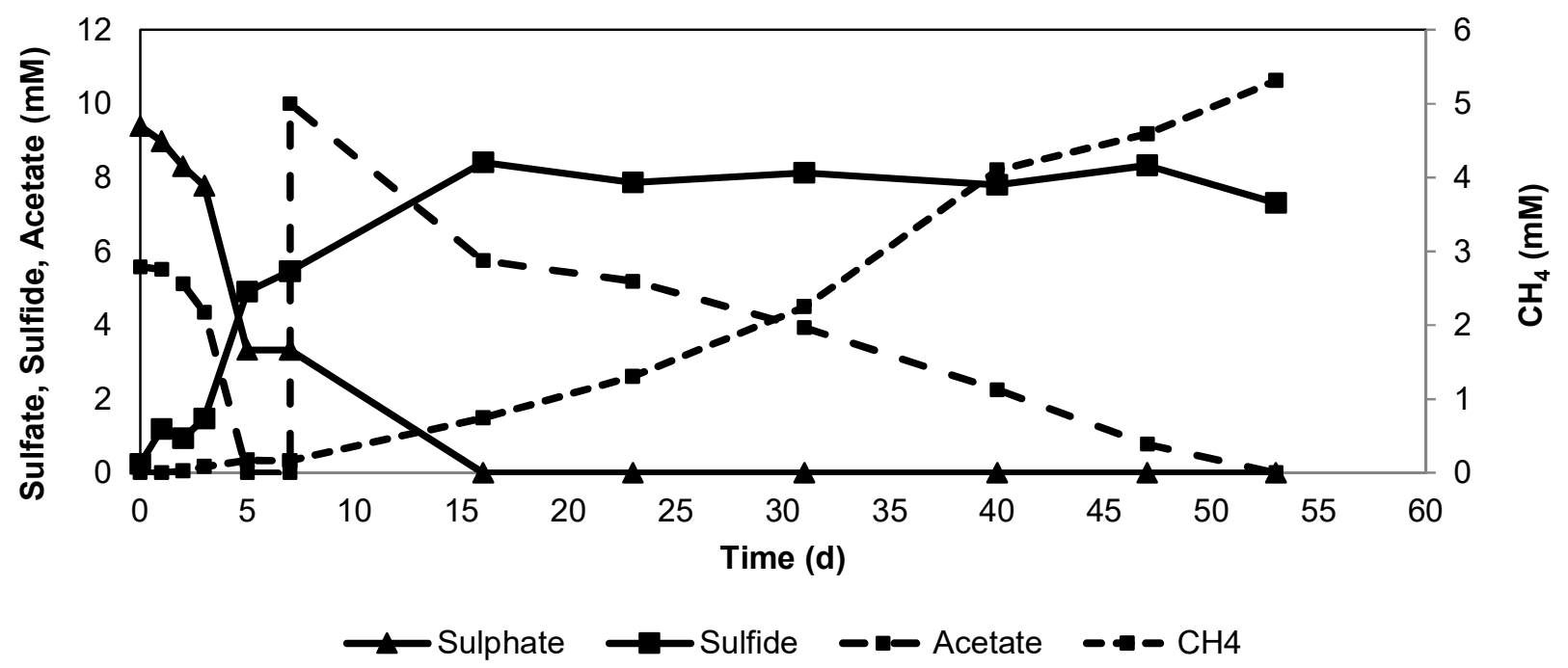


Chapter 2 


\section{Chapter 3}

Butyrate degradation by sulfatereducing and methanogenic communities in anoxic sediments of Aarhus Bay, Denmark 


\section{Abstract}

The bacterial and archaeal communities enriched with butyrate in sediment slurries taken from different biogeochemical zones of Aarhus Bay, Denmark were analyzed. Sulfate was added at different concentrations $(0,3,20 \mathrm{mM})$ to the sediment slurries and the slurries were incubated at $25^{\circ} \mathrm{C}$ and at $10^{\circ} \mathrm{C}$. An immediate start of methanogenesis in sulfate zone slurries and sulfate reduction in methane zone slurries was observed. During butyrate conversion, sulfate reduction and methanogenesis occurred simultaneously. Desulfobacteraceae, Desulfovibrionaceae, Desulfobulbaceae, Syntrophomonadaceae and Clostridiales members involved in sulfate-dependent butyrate conversion in $25^{\circ} \mathrm{C}$ slurries. The obligate syntroph Syntrophomonas was enriched both in sulfate-amended and sulfate-free slurries indicating the co-occurrence of sulfate-dependent and syntrophic butyrate conversion. The low temperature sulfate-amended slurries contained mainly Desulfobacteraceae and uncultured Firmicutes, whereas sulfate-free slurries consisted of sequences related to uncultured Firmicutes and Desulfobulbaceae. Archaeal community analysis revealed the dominance of Methanomicrobiaceae in the slurries. Methanosaetaceae reached high abundance in the absence of sulfate, whereas the presence of Methanosarcinaceae was independent of the sulfate concentration, temperature and the origin of sediment. This study shows that sulfate reducers, syntrophs and methanogens are present together in the upper and lower parts of marine sediments and cooperate in the conversion of butyrate. 


\subsection{Introduction}

Coastal marine ecosystems receive regular input of organic matter and nutrients from primary production of plankton, macroalgae and vascular plants, influx of rivers and remineralization of organic carbon (Jørgensen, 2006; Canfield et al., 2006). Most particulate organic matter is rapidly deposited on the coastal shelf (Jørgensen, 1983). High microbial activity in the sediment layers leads to the formation of distinct biogeochemical zones. The depth range of each zone varies strongly depending on chemical changes in the sediment pore water, the rates of sediment accumulation and replenishment of electron acceptors from overlying seawater (Jørgensen, 1983, Mitterer, 2010). In coastal marine sediments, the thickness of the oxic surface layer is can be just a few mm (Canfield et al., 2006). Where oxygen is depleted, the sediment becomes anoxic. In the anoxic part of the sediment, nitrate, iron, manganese, sulfate and carbon dioxide, in an order of decreasing energy gain, serve as terminal electron acceptors for the mineralization processes. In marine sediments, sulfate reduction is the predominant pathway, while methanogenesis becomes important in zones where sulfate is depleted (Jørgensen, 1982; Holmkvist et al., 2011; Bowles et al., 2014).

Anaerobic degradation of organic matter in sediments is a complex, sequential process involving a variety of physiologically different microorganisms (Jørgensen, 2006). The first step is an extracellular hydrolytic conversion of polymers, followed by fermentation of the monomers and oligomers to reduced organic compounds such as short chain fatty acids, alcohols, formate, $\mathrm{H}_{2}$ and $\mathrm{CO}_{2}$. Organic acids and alcohols are further degraded to acetate, formate, $\mathrm{H}_{2}$ and $\mathrm{CO}_{2}$. In general, sulfate reduction and methanogenesis are the terminal steps in the overall anaerobic degradation process (Schink and Stams, 2013; Muyzer and Stams, 2008; Stams and Plugge, 2009). Both sulfate reduction and methanogenesis are considered to be mutually exclusive in most environmental settings and controlled mainly by the amount of available sulfate (Roussel et al., 2015; O'Sullivan et al., 2013). When the concentration of sulfate is high, which is the case for marine environments, sulfate reducers are the main utilizers of hydrogen, formate and acetate (Hoehler et al., 1998; Bowles et al., 2014). They outcompete methanogens due to their higher affinity and lower threshold values for common substrates.

In sulfate-limited or sulfate-depleted sediments, organic matter is degraded through methanogenesis. In this case, hydrogen, formate and acetate that are released as end products of organic carbon degradation are converted to $\mathrm{CH}_{4}$ and $\mathrm{CO}_{2}$ by methanogens. In this way, a syntrophic relationship is established between microorganisms that degrade organic compounds and methanogens (Schink and Stams, 2013; McInerney et al., 2008). Syntrophic fatty acids 
degradation is known as a rate limiting step of organic carbon degradation (Schink and Stams, 2013).

In the past, methanogenesis and sulfate reduction were considered to be separated in marine sediments based on sulfate availability (Cappenberg, 1974; Mountfort and Asher, 1981). Later, both sulfate reduction and methanogenesis were reported to occur simultaneously in anoxic marine environments where input of organic carbon is high (Maltby et al., 2016; Mitterer, 2010; Jørgensen and Parkes, 2010; Oremland, 1982; Senior et al., 1982; Holmer and Kristensen, 1994). In such environments, the use of non-competitive methylated substrates by methanogens was suggested to enable co-habitation of both functional groups of microbes (Visscher et al., 2003; Oremland and Polcin, 1982). However, several studies demonstrated the consumption of common substrates, $\mathrm{H}_{2}$ and acetate, by both microbial groups in sulfate-rich sediments (Oremland and Taylor, 1978; Senior et al., 1982; Finke et al., 2007b). Sulfate reducers were detected in the methane zone in comparable numbers to the sulfate zone of Black Sea and Aarhus Bay (Leloup et al., 2007, 2009). The niche differentiation of the two groups of microbes is not fully understood. Thus far, syntrophic degradation of fatty acids in marine environments has received little attention.

Butyrate can be metabolized by direct sulfate reduction to acetate and $\mathrm{CO}_{2}$ (Widdel, 1988; Muyzer and Stams, 2008) or by syntrophic associations of butyrate degrading bacteria with $\mathrm{H}_{2}$ and/or acetate consuming sulfate reducer or methanogen (Table 1). Butyrate degradation coupled to sulfate reduction occurs in anaerobic ecosystems containing high sulfate, such as marine sediments, anaerobic digesters and aquifers (Jørgensen, 1982; Banat and Nedwell, 1983; Alphenaar et al., 1993; Visser et al., 1993; Kleikemper et al., 2002; Roest et al., 2005; Struchtemeyer et al., 2011). Syntrophic butyrate degraders were detected in similar quantities in the presence and absence of sulfate in anaerobic bioreactors (Roest et al., 2005) and others reported that syntrophic butyrate degraders were efficiently able to compete with sulfate reducers even in excess sulfate (Visser et al., 1993; Rebac et al., 1996). Methanogenesis has been observed in sulfate zones of marine sediments (Parkes et al., 1990; Kendall et al., 2006). Kendall and colleagues (2006) detected marine butyratedegrading syntrophs in the sulfate zone and suggested that syntrophic interactions constitute an enormous methane source in marine sediments. Similarly, high methane concentrations were observed under high sulfate conditions in a hydrocarbon-contaminated aquifer where the investigators concluded that butyrate was metabolized mainly syntrophically (Kleikemper et al., 2002). Several other studies reported the existence of Syntrophus and Syntrophomonas genera in 
different sulfate containing environments as a proof of syntrophic butyrate degradation (Dar et al., 2005; Winderl et al., 2008).

Table 1. Overview of reactions examined in this study. $\Delta \mathrm{G}$ values were obtained from Thauer et al., 1977.

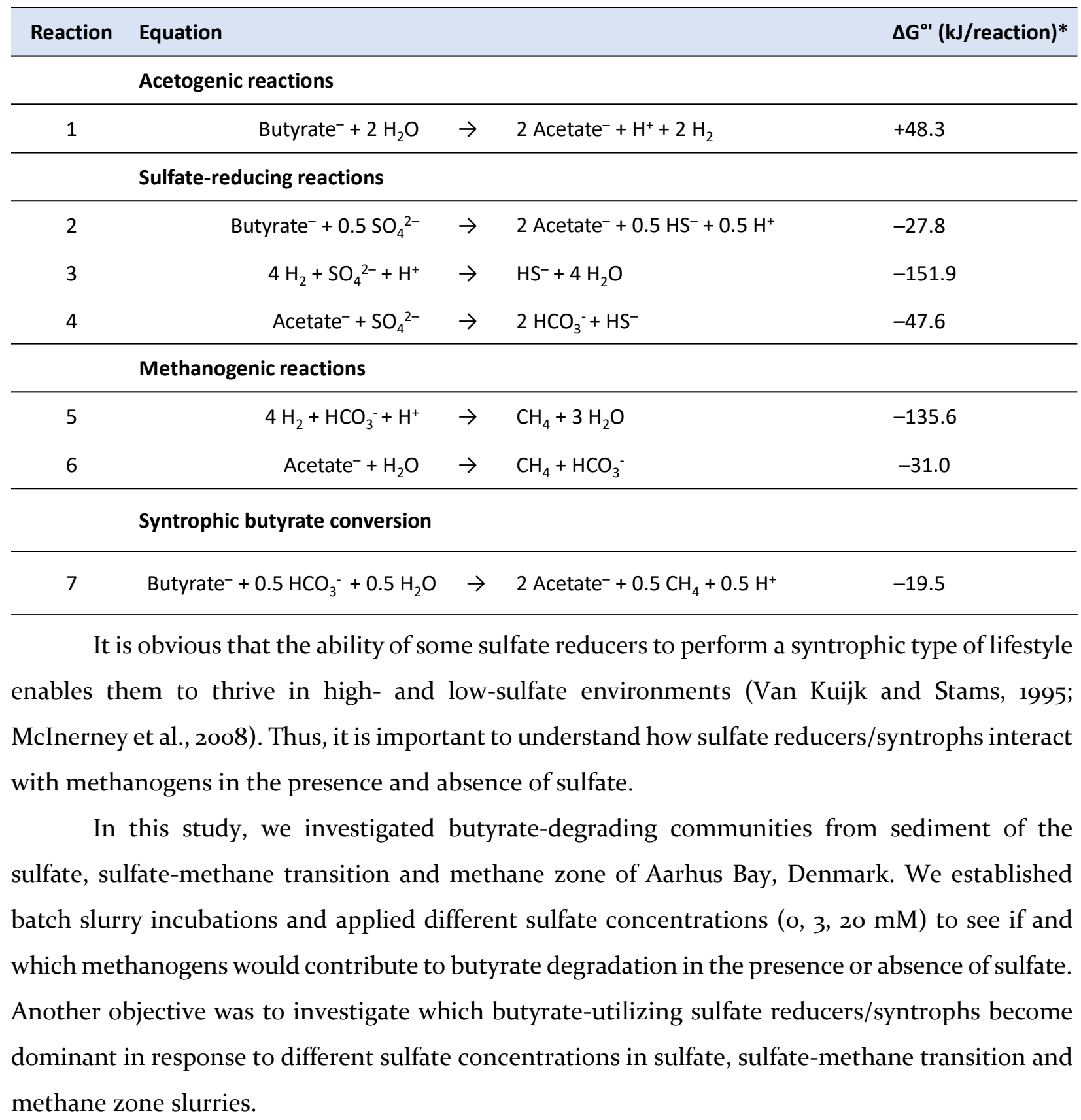




\subsection{Materials and methods}

\subsubsection{Sediment sampling}

Sediment was collected during a research cruise in May 2011, in Aarhus Bay, Denmark. The studied site, Station $\mathrm{Ml}$, is located in the central part of the Bay, at position $56^{\circ} \mathrm{O} 7^{\prime} \mathrm{O} 66^{\prime \prime} \mathrm{N}, 1^{\circ} 20^{\prime} 793^{\prime \prime} \mathrm{E}$. The in situ temperature was $\sim 9^{\circ} \mathrm{C}$ and the water depth was $15 \mathrm{~m}$. Two 3-m-long gravity cores were retrieved; one of them was sectioned in $10 \mathrm{~cm}$ depth intervals for physical, chemical and molecular analyses and the other one was kept intact in the core liners, in sealed gas-tight plastic bags containing AnaeroGen sackets (Oxoid xx) at $4{ }^{\circ} \mathrm{C}$ until further processed.

\subsubsection{Sediment pore water analysis}

Methane, sulfate and sulfide analysis from sediment pore water were performed on the sampling day at the laboratories of Center for Geomicrobiology, Aarhus University. Methane concentration was determined in a sediment sample of $2.5 \mathrm{~cm}^{3}$. The sample was taken immediately after retrieval of the sediment core and transferred to a $25 \mathrm{ml}$ serum vial with $4 \mathrm{ml} 2.5 \% \mathrm{NaOH}$, immediately capped with a butyl rubber stopper, crimp-sealed, shaken vigorously and stored upside down until measurement. After equilibration ( an hour), the headspace composition was analyzed on a gas chromatograph (5890A, Hewlett Packard) equipped with a packed stainless steel Porapak-Q column (6 ft., 0.125 in., 8o/10o mesh, Agilent Technology) and a flame ionization detector. Helium was used as a carrier gas at a flow rate of $30 \mathrm{ml} / \mathrm{min}$.

Sulfate and sulfide were quantified in pore water extracted directly from sediment. Rhizon samplers (Rhizosphere Research Products, Wageningen, Netherlands) were inserted into the core at $10 \mathrm{~cm}$ intervals through holes that were drilled in the plastic corer and pore water was collected in $5 \mathrm{ml}$ vacuumed plastic syringe connected to Rhizon samplers. For sulfate analysis, subsamples of pore water was degassed with oxygen-free $\mathrm{CO}_{2}$ to lower the $\mathrm{pH}$ and remove hydrogen sulfide. Sulfate measurement was done using Dionex (Sunnyvale, CA) ion chromatography system 50 equipped with AS18 column. The eluent was 20-32 $\mathrm{mM} \mathrm{KOH}$ at a flow rate of $1 \mathrm{ml} / \mathrm{min}$. For sulfide analysis, subsamples were mixed 1:1 (v/v) with 5\% (w/v) zinc acetate. Samples were diluted in MiliQ water and diamine reagent was added. After color development, sulfide concentration was measured by microplate reader at $670 \mathrm{~nm}$. 


\subsubsection{Sediment slurry incubations}

Sediments from three different biogeochemical zones were used to establish replicate sediment slurries. Zones were defined based on sulfate and methane concentrations determined using pore water extracted from sediment during sampling cruise. The sulfate concentration decreased from $18.5 \mathrm{mM}$ at $15 \mathrm{~cm}$ of the core to a low background value at $170 \mathrm{~cm}$. Methane increased steeply with depth below $120 \mathrm{~cm}$ and reached a plateau of $2 \mathrm{mM}$ at $225 \mathrm{~cm}$ (Fig S1). The sediment core was divided into three pieces representing the sulfate zone (SR) $(15-120 \mathrm{~cm})$, the sulfate-methane transition zone (SMTZ) $(120-170 \mathrm{~cm})$ and the methane zone $(\mathrm{MZ})(170-300 \mathrm{~cm})$.

Stored sediment cores were processed under aseptic and anaerobic conditions in the laboratory. Subsamples representing a particular biogeochemical zone were mixed in an anaerobic chamber and used as inoculum for sediment slurry enrichments. $100 \mathrm{ml}$ of the homogenized sediment from each zone was mixed with $300 \mathrm{ml}$ of anaerobic mineral salts medium in $1 \mathrm{~L}$ serum bottles. The medium composition was as followed (g/L): $\mathrm{KH}_{2} \mathrm{PO}_{4}(0.41), \mathrm{Na}_{2} \mathrm{HPO}_{4} \cdot 2 \mathrm{H}_{2} \mathrm{O}$ (o.53), $\mathrm{NH}_{4} \mathrm{Cl}$ (o.3), $\mathrm{CaCl}_{2} .2 \mathrm{H}_{2} \mathrm{O}$ (o.11), $\mathrm{MgCl}_{2} .6 \mathrm{H}_{2} \mathrm{O}$ (3), $\mathrm{NaHCO}_{3}$ (4), $\mathrm{Na}_{2} \mathrm{~S} .9 \mathrm{H}_{2} \mathrm{O}$ (o.024), KCL (o.5), $\mathrm{NaCl}$ (25). The medium was supplemented with $1 \mathrm{ml} /$ liter of acid trace element solution $(50 \mathrm{mM} \mathrm{HCl}, 1$ $\mathrm{mM} \mathrm{H}_{3} \mathrm{BO}_{3}$, $0.5 \mathrm{mM} \mathrm{MnCl}_{2}, 7.5 \mathrm{mM} \mathrm{FeCl}_{2}$, 0.5 $\mathrm{mM} \mathrm{CoCl}_{2}$, o.1 $\mathrm{mM} \mathrm{NiCl}_{2}$, $0.5 \mathrm{mM} \mathrm{ZnCl}_{2}$ ), $1 \mathrm{ml} /$ liter of alkaline trace element solution (1o mM NaOH, o.1 $\mathrm{mM} \mathrm{Na}_{2} \mathrm{SeO}_{3}, 0.1 \mathrm{mM} \mathrm{Na}_{2} \mathrm{WO}_{4}, 0.1 \mathrm{mM} \mathrm{Na}_{2} \mathrm{MoO}_{4}$ ) and $10 \mathrm{ml} /$ liter vitamin solution (Biotin $20 \mathrm{mg} / \mathrm{l}$, Nicotinamid $200 \mathrm{mg} / \mathrm{l}$, p-Aminobenzoic acid 100 $\mathrm{mg} / \mathrm{l}$, Thiamin $200 \mathrm{mg} / \mathrm{l}$, Panthotenic acid $100 \mathrm{mg} / \mathrm{l}$, Pyridoxamine $500 \mathrm{mg} / \mathrm{l}$, Cyanocobalamine 100 $\mathrm{mg} / \mathrm{l}$, Riboflavin $100 \mathrm{mg} / \mathrm{l}$ ). Bottles were closed with butyl rubber stoppers and the headspace was exchanged with $\mathrm{N}_{2} / \mathrm{CO}_{2}(80: 20 \%, \mathrm{v} / \mathrm{v})$. $10 \mathrm{mM}$ butyrate was used as carbon source with and without $20 \mathrm{mM}$ sulfate in sulfate zone and methane zone slurries, and with $3 \mathrm{mM}$ and $20 \mathrm{mM}$ sulfate for sulfate-methane transition zone slurries as electron acceptor. Control bottles were prepared in the same manner, without addition of butyrate. One set of the bottles representing each condition in duplicate was incubated at $10^{\circ} \mathrm{C}$ to mimic in situ temperature (Dale et al., 2008) and the other set was kept at $25^{\circ} \mathrm{C}$ statically throughout the experiment. Regular liquid and gas sampling was performed to monitor substrate consumption, product formation and to carry out molecular analysis. Regular additions of butyrate and/or sulfate were done as soon as they were depleted to maintain the slurry conditions same. 


\subsubsection{Analytical methods}

$\mathrm{CH}_{4}$ in the headspace of slurries was analyzed by gas chromatography with a Shimadzu GC-14B (Shimadzu, Kyoto, Japan) equipped with a packed column (Molsieve 13X, 6o-80 mesh, 2 m length, $3 \mathrm{~mm}$ internal diameter; Varian, Middelburg, The Netherlands) and a thermal conductivity detector set at $70 \mathrm{~mA}$. The oven temperature and the injector temperatures were both $100^{\circ} \mathrm{C}$. The detector temperature was $150^{\circ} \mathrm{C}$. Argon was the carrier gas at a flow rate of $30 \mathrm{ml} \mathrm{min}$.

Volatile fatty acids from centrifuged (10,000 X $g$, $10 \mathrm{~min})$ samples of the sediment slurries were analyzed by HPLC system equipped with a Varian column (Metacarb $67 \mathrm{H} 300 x 6.5 \mathrm{~mm}$, Middelburg, The Netherlands) connected to a UV and Refractive Index (RI) detector. $10 \mathrm{mM}$ sulfuric acid was used as eluent and sodium crotonate as internal standard. The flow rate was o.8 $\mathrm{ml} \mathrm{min}{ }^{-1}$ and analyses were carried out at $30^{\circ} \mathrm{C}$. Data analyses were performed using ChromQuest (Thermo Scientific, Waltham, MA, USA) and Chromeleon software (Thermo Scientific, Waltham, MA).

Sulfate concentrations were analyzed by Ion Chromatography system equipped with an AS22 column (4x250 mm) and ED 40 electrochemical detector (Dionex, Sunnyvale, CA). The eluents were $1.7 \mathrm{mM} \mathrm{NaHCO}_{3}$ and $1.8 \mathrm{mM} \mathrm{Na}_{2} \mathrm{CO}_{3}$. The analyses were conducted with a flow rate of $1.2 \mathrm{ml} \mathrm{min}^{-1}$ at $35^{\circ} \mathrm{C}$. Sodium bromide was used as internal standard.

Sulfide measurements were done using methylene blue method (Cline, 1969). Samples were 1:1 diluted with $5 \%(\mathrm{w} / \mathrm{v})$ zinc acetate solution, directly after sampling, to precipitate all sulfide. 4.45 $\mathrm{ml}$ of deionized water, $500 \mu \mathrm{l}$ of reagent A ( $2 \mathrm{~g} / \mathrm{l}$ dimethylparaphenylenediamine and $200 \mathrm{ml} / \mathrm{l}$ $\left.\mathrm{H}_{2} \mathrm{SO}_{4}\right)$ and $50 \mu \mathrm{l}$ of reagent $\mathrm{B}\left(1 \mathrm{~g} / \mathrm{l} \mathrm{Fe}\left(\left(\mathrm{NH}_{4}\right)\left(\mathrm{SO}_{4}\right)\right)_{2} \cdot 12 \mathrm{H}_{2} \mathrm{O}\right.$ and $\left.0.2 \mathrm{ml} / \mathrm{l} \mathrm{H}_{2} \mathrm{SO}_{4}\right)$ was added concurrently and mixed. The solution was stored for at least 10 minutes at room temperature. After color development, the concentration was measured on a MERCK Spectroquant ${ }^{\circledast}$ Multy colorimeter (Merck Millipore, Darmstadt, Germany) at 67onm. Demi-water was used as a blank.

\subsubsection{DNA extraction}

Genomic DNA was extracted from the sediment and enrichment slurry samples that were taken at different time points using the FastDNA SPIN Kit for Soil (MP Biomedicals, OH) according to manufacturer's protocol. Adaptation of the commercial protocol was carried out to increase the DNA yield. $5 \mathrm{ml}$ sediment or slurry sample were suspended in $10 \mathrm{ml}$ of phosphate-buffered saline (PBS), sonicated at low power to detach cells from the solid phase and was centrifuged at $4700 \mathrm{~g}$ 
for $20 \mathrm{~min}$. The supernatant was discarded and remaining pellet was re-suspended in $10 \mathrm{ml} 0.5 \mathrm{M}$ EDTA, pH 8, and incubated overnight at $4^{\circ} \mathrm{C}$ to dissolve humic substances. After incubation, the suspension was centrifuged at $4700 \mathrm{~g}$ for $10 \mathrm{~min}$., washed with PBS and DNA extraction procedures were applied to the pellet. The DNA was quantified with a Nanodrop ND-10oo spectrophotometer (Nanodrop Technologies, Wilmington, DE).

\subsubsection{DGGE analysis}

DNA from one replicate of all the slurries was used for DGGE analysis. Amplification of the V6-V8 region of the bacterial $16 \mathrm{~S}$ rRNA was performed using the primer pair F-968-GC (5'AACGCGAAGAACCTTAC-3') and R-1401 (5'-CGGTGTGTACAAGACCC-3') (Nübel et al., 1996). PCR was performed using GoTaq DNA Polymerase Kit (Promega, Madison, WI). The $50 \mu$ l reaction mixture contained $10 \mu \mathrm{l}$ GoTaq buffer, $10 \mu \mathrm{M}$ of each primer, $1 \mu \mathrm{l}$ of dNTP solution and $1 \mu \mathrm{l}$ of genomic DNA. PCR was carried out by using an initial denaturation step at $95^{\circ} \mathrm{C}$ for $5 \mathrm{~min}$, followed by 35 cycles of denaturation step at $95^{\circ} \mathrm{C}$ for $30 \mathrm{~s}$, annealing step at $52^{\circ} \mathrm{C}$ for $40 \mathrm{~s}$, elongation step at $72^{\circ} \mathrm{C}$ for 6 o s., and a final elongation step at $72^{\circ} \mathrm{C}$ for $10 \mathrm{~min}$. The $\mathrm{V}_{3}$ region of the archaeal $16 \mathrm{~S}$ rRNA gene fragments was amplified with primers ARC344f-GC (5'-ACGGGGYGCAGCAGGCGCGA-3') and ARC519r (5'-GWATTACCGCGGCKGCTG-3') (Yu et al., 2008) using the GoTaq DNA Polymerase Kit (Promega, Madison, WI). PCR conditions were as followed: an initial denaturation step at $95^{\circ} \mathrm{C}$ for $5 \mathrm{~min}$; followed by 10 cycles of denaturation step at $95^{\circ} \mathrm{C}$ for $30 \mathrm{~s}$, annealing step at $61^{\circ} \mathrm{C}$ for $40 \mathrm{~s}(-$ $0.5^{\circ} \mathrm{C} /$ cycle), elongation step at $72^{\circ} \mathrm{C}$ for $45 \mathrm{~s} ; 25$ cycles of denaturation step at $95^{\circ} \mathrm{C}$ for $30 \mathrm{~s}$, annealing step at $5^{6 \circ} \mathrm{C}$ for $40 \mathrm{~s}$, elongation step at $72^{\circ} \mathrm{C}$ for $45 \mathrm{~s}$; and final elongation step at $72^{\circ} \mathrm{C}$ for 10 min. Forward primers had a GC clamp of 40 bp attached to the 5' end (Yu et al., 2008). The presence and sizes of the amplification products were determined by agarose (1\%) gel electrophoresis using the Smart Ladder (Eurogentec) as molecular weight marker. SYBR Safe ${ }^{\circledR}$ stained gel pictures were digitally recorded.

DGGE was performed as described by Muyzer et al. (1993) using the DCode system (BioRad Laboratories, Hercules, CA). A denaturing gradient of $30-60 \%$ for bacteria and $40-60 \%$ for archaea were used as recommended by Yu et al (2008). Gels were initially run at $200 \mathrm{~V}$ for $10 \mathrm{~min}$ to facilitate the access of PCR products into the denaturing gradient gel, and then at a constant voltage of $100 \mathrm{~V}$ for $16 \mathrm{~h}$ at $60^{\circ} \mathrm{C}$ in $1 \mathrm{X}$ TAE buffer. After electrophoresis, the gels were stained with $\mathrm{AgNO}_{3}$ according to Sanguinetti et al. (1994). DGGE gels were scanned with a BioRad GS-8oo Calibrated Densitometer and analyzed using the BioNumerics ${ }^{\circledR}$ software version 4.6 (Applied 
Maths, Sint-Martens-Latem, Belgium). The Dice coefficient was used to determine the similarity between DGGE fingerprints by calculating the similarity indices of the densitometric curves of the profiles and dendrograms for bacterial and archaeal DGGE profiles were created using Unweighted Pair Group Method Analysis (UPGMA).

\subsubsection{S rRNA gene amplicon pyrosequencing}

Bacterial 16S rRNA gene fragments were amplified using barcoded primers covering the $\mathrm{V}_{\mathbf{1}}-\mathrm{V}_{\mathbf{2}}$ region of the bacterial $16 \mathrm{~S}$ rRNA gene. The forward primer consisted of the $27 \mathrm{~F}-\mathrm{DegS}$ primer $\left(5^{\prime}-\right.$ GTTYGATYMTGGCTCAG- 3') (van den Bogert et al., 2011) appended with the titanium sequencing adaptor A (5'- CCATCTCATCCCTGCGTGTCTCCGACTCAG- 3') and an 8 nucleotide sample specific barcode (Hamady et al., 2008) at the $5^{\prime}$ end. An equimolar mix of two reverse primers was used i.e. 338RI (5' - GCWGCCTCCCGTAGGAGT- 3') and 338RII (5' - GCWGCCACCCGTAGG TGT$3^{\prime}$ ) (Daims et al., 1999) that carried the titanium adaptor B (5' - CCTATCCCCTGTGTGCCTTGGCAG TCTCAG- $3^{\prime}$ ) at the $5^{\prime}$ end. Sequences of both titanium adaptors were purchased from GATC Biotech (Konstanz, Germany).

Genomic DNA was diluted to a concentration of $20 \mathrm{ng} / \mu \mathrm{l}$ based on Qubit $^{\circledast}$ 2.0 fluorometer readings. PCR was performed using a GSooo1 thermocycler (Gene Technologies, Braintree, United Kingdom). 10o $\mu$ l PCR mixture contained $20 \mu$ l of $5 \times$ HF buffer (Finnzymes, Vantaa, Finland), $2 \mu l$ PCR Grade Nucleotide Mix (Roche Diagnostic GmbH, Mannheim, Germany), $1 \mu$ l of Phusion hot start II High-Fidelity DNA polymerase (2U/ $\mu \mathrm{l}$; Finnzymes), $500 \mathrm{nM}$ of the reverse primer mix and the forward primer (Biolegio BV, Nijmegen, The Netherlands), $2 \mu \mathrm{l}$ (40 ng) template, and $65 \mu \mathrm{l}$ nuclease free water. PCR was performed using the following conditions: $98^{\circ} \mathrm{C}$ for $30 \mathrm{~s}$ to activate the polymerase, followed by 30 cycles of denaturation at $98^{\circ} \mathrm{C}$ for $10 \mathrm{~s}$, annealing at $56^{\circ} \mathrm{C}$ for $20 \mathrm{~s}$, elongation at $72^{\circ} \mathrm{C}$ for $20 \mathrm{~s}$, and a final extension at $72^{\circ} \mathrm{C}$ for $10 \mathrm{~min}$. Five $\mu \mathrm{l}$ of the PCR products (approximately $450 \mathrm{bp}$ ) were analyzed by $1 \%(\mathrm{w} / \mathrm{v})$ agarose gel electrophoresis, containing $1 \times$ SYBR Safe (Invitrogen, Carlsbad, CA) to verify the right length of the amplicons. PCR products were purified using High Pure PCR Cleanup Micro Kit (Roche Diagnostics, Germany) according to the manufacturer's instructions and DNA concentration of gel-purified amplicons was measured by Qubit $^{\circledR}$ 2.0 Fluorometer (Life Technologies, Germany). Purified PCR products were mixed in equimolar amounts and run again on an agarose gel. This was followed by excision of bands and purification using a DNA gel extraction kit (Millipore, Massachusetts, o1821). DNA concentration was measured using Qubit and $1 \mu \mathrm{l}$ purified equimolar pool of PCR product was analyzed on $1 \%$ 
agarose gel. Samples were analyzed by pyrosequencing using an FLX genome sequencer in combination with titanium chemistry (GATC Biotech AG, Konstanz, Germany).

\subsubsection{Analysis and interpretation of the pyrosequencing data}

Pyrosequencing data was analyzed using the Quantitative Insights Into Microbial Ecology (QIIME) 1.8.o pipeline (Caporaso et al., 2010). Sequence reads were initially filtered using default parameters and denoised (Bragg et al., 2012) for removing low quality reads. UCHIME was used to remove chimeric sequences from pre-processed data from the dataset (Edgar, 2010). From the remaining set of high quality 16S rRNA gene sequences, operational taxonomic units (OTUs) were defined at a 97\% identity level. A representative sequence from each OTU was aligned using PyNAST (DeSantis et al., 2006). The taxonomic affiliation of each OTU was determined at an identity threshold of 97\% using UCLUST algorithm (Edgar, 2010) and SILVA 111 database as a reference (Pruesse et al., 2007). The relative amount of reads of every OTU to the total amount of reads per sample was quantified and the average relative amount of reads per representative OTU of each slurry sample was calculated.

\subsubsection{Illumina MiSeq analysis of archaeal community}

Extracted DNA from the samples taken on the last incubation day from all slurries was used for archaeal community analysis. Barcoded amplicons were generated using a two-step PCR method that was shown to reduce the impact of barcoded primers on the outcome of microbial profiling (Berry et al., 2011). First amplification of archaeal 16S rRNA gene fragments was done using primers 518F (5'-CAGCMGCCGCGGTAA-3') (Wang and Qian, 2009) and 905R (5'CCCGCCAATTCCTTTAAGTTTC-3') (Kvist et al., 2007). PCRs were performed using a SensoQuest Labcycler (Göttingen, Germany). PCR amplification was performed in a total volume of $50 \mu \mathrm{l}$ containing $500 \mathrm{nM}$ of each forward and reverse primer (Biolegio BV), 1 unit of Phusion DNA polymerase (Thermo Scientific), 10 $\mu$ l of HF-buffer, $200 \mu \mathrm{M}$ dNTP mix, made to a total volume of $50 \mu$ with nuclease free sterile water. The PCR program was as followed: denaturing at $98^{\circ} \mathrm{C}$ for 30 s, followed by 25 cycles of denaturing at $98^{\circ} \mathrm{C}$ for $10 \mathrm{~s}$, annealing at $60^{\circ} \mathrm{C}$ for $20 \mathrm{~s}$, extension at $72^{\circ} \mathrm{C}$ for $20 \mathrm{~s}$, followed by a final extension step at $72^{\circ} \mathrm{C}$ for $10 \mathrm{~min}$. PCR products were confirmed by agarose gel electrophoresis containing $1 \times$ SYBR Safe (Invitrogen, Carlsbad, CA). A second PCR was employed to add an 8 nucleotide sample-specific barcode to the 5'- and 3'-end of PCR products 
(Ramiro-Garcia et al., 2016). Each PCR reaction with a final volume of $100 \mu \mathrm{L}$ contained $5 \mu \mathrm{L}$ of the first PCR product, $5 \mu \mathrm{L}$ each of $10 \mu \mathrm{M}$ barcoded forward and reverse primers (Biolegio BV), $200 \mu \mathrm{M}$ PCR Grade dNTP mix (Roche Diagnostics), 2 units of Phusion ${ }^{\circledR}$ Hot Start II High-Fidelity DNA polymerase (Thermo Scientific) and $20 \mu \mathrm{L}$ of HF buffer (Finnzymes, Vantaa, Finland). Amplification consisted of an initial denaturation at $98{ }^{\circ} \mathrm{C}$ for $30 \mathrm{~s} ; 5$ cycles of denaturation at $98{ }^{\circ} \mathrm{C}$ for $10 \mathrm{~s}$, annealing at $52{ }^{\circ} \mathrm{C}$ for $20 \mathrm{~s}$, and elongation at $72{ }^{\circ} \mathrm{C}$ for $20 \mathrm{~s}$; and a final extension at $72{ }^{\circ} \mathrm{C}$ for $10 \mathrm{~min}$. PCR products were purified using the HighPrep ${ }^{\text {TM }}$ PCR clean-up system (MagBio Genomics Inc., Gaithersburg, MD). Amplicons were quantified using Qubit (Invitrogen, Bleiswijk, The Netherlands). Afterwards, barcoded samples were pooled in equimolar quantities, purified using the MagBio HighPrep PCR- 96 well protocol and then quantified using Qubit. Samples were sequenced at GATC Biotech AG (Konstanx, Germany) by Illumina Miseq sequencing.

16S rRNA gene sequencing data was analysed using NG-Tax, an in-house pipeline (RamiroGarcia et al., 2016). Paired-end libraries were filtered to contain only read pairs with perfectly matching barcodes, and those barcodes were used to demultiplex reads by sample. Finally operational taxonomic units (OTUs) were defined using an open reference approach, and taxonomy was assigned to those OTUs using the SILVA $16 \mathrm{~S}$ rRNA gene reference database (Quast et al., 2013). Microbial composition plots were generated using a workflow based on Quantitative Insights Into Microbial Ecology (QIIME) v1.2 (Caporaso et al., 2010).

\subsubsection{Statistical analysis}

Redundancy analysis was performed as implemented in the CANOCO 5 software package (Biometris, Wageningen, The Netherlands) in order to assess to what extent experimental variables influenced the microbial community composition. The experimental variables tested were the incubation temperature, total concentrations of sulfate, butyrate, acetate and methane consumed/produced by the end of the incubations. A Monte Carlo permutation test based on 499 random permutations were used to determine which of the experimental variables significantly contributed to the observed variance in the composition of microbial communities at the order (for Bacteria) and family level (for Archaea). Orders and families of at least $5 \%$ relative abundance in any sample were included in the analysis. The community structure was visualized via ordination triplots with scaling focused on intersample differences.

Correlations between bacterial and archaeal groups and experimental parameters were determined by means of the two-tailed Spearman's Rank Order Correlation test using the statistical 
software SPSS Statistics (IBM SPSS Statistics, Version 22, IBM Corp., Armonk, NY). A statistical significance level of $5 \%$ was applied.

\subsection{Results}

\subsubsection{Sampling site geochemistry}

Aarhus Bay is a shallow semi-enclosed embayment on the transition between the North Sea and Baltic Sea and characterized by elevated primary production during the summer months (Glud et al., 2003). Two 3-m-long gravity cores were retrieved and one of the cores was used to carry out pore water analysis to measure methane, sulfate and sulfide throughout the core on the same day. According to the pore water analysis results, the sulfate zone was determined to be located between $\mathrm{o} \mathrm{cm}$ and $120 \mathrm{~cm}$, sulfate-methane transition zone (SMTZ) between $120 \mathrm{~cm}$ and $170 \mathrm{~cm}$ and methane zone between $170 \mathrm{~cm}$ and $300 \mathrm{~cm}$ (Fig S1).

\subsubsection{Sediment slurry enrichments}

Sediment slurries consisting of the sediment sample taken from the sulfate, sulfate-methane transition and methane zones were analyzed for substrate consumption and product formation at different time points throughout the incubation period. Total amounts of the butyrate and sulfate consumed and acetate, sulfide and methane produced in all slurries were listed in Table S1.

\subsubsection{Sulfate zone sediment slurries}

Sulfate zone sediment slurries were incubated for 514 days at $25^{\circ} \mathrm{C}$ and $10^{\circ} \mathrm{C} \mathrm{(Fig} \mathrm{1).} \mathrm{Conversion} \mathrm{of}$ butyrate in sulfate-containing slurries started after 12 days of incubation (Fig 1B, 1D). Repeated additions of butyrate and sulfate caused a steady increase in acetate and sulfide. Methane formation was observed after 309 days at $25^{\circ} \mathrm{C}$ and after 260 days at $10^{\circ} \mathrm{C}$, and increased with time (Fig $\left.1 \mathrm{~B}, 1 \mathrm{D}\right)$.

In the methanogenic slurries, where no additional sulfate was added, about $1 \mathrm{mM}$ sulfate was measured at the start of the incubation, which presumably originated from the sediment (Fig $1 \mathrm{~A}, 1 \mathrm{C}$ ). In the first $4 \mathrm{O}$ days of incubation at $25^{\circ} \mathrm{C}$, conversion of butyrate coupled to sulfate reduction occurred. Methane formation started on day 50, after sulfate had been depleted, indicating syntrophic butyrate conversion. Repeated additions of butyrate yielded high methane and acetate accumulation. Acetate was gradually consumed after day 203 at $25^{\circ} \mathrm{C}$, and decreased to 2359 
$\mu \mathrm{mol} /$ bottle from $2475^{2} \mu \mathrm{mol} /$ bottle on day 430 (Fig $1 \mathrm{~A}$ ). Butyrate conversion in the $10^{\circ} \mathrm{C}$ slurry was rather slow $\left(\right.$ Fig $\left._{1} \mathrm{C}\right)$.
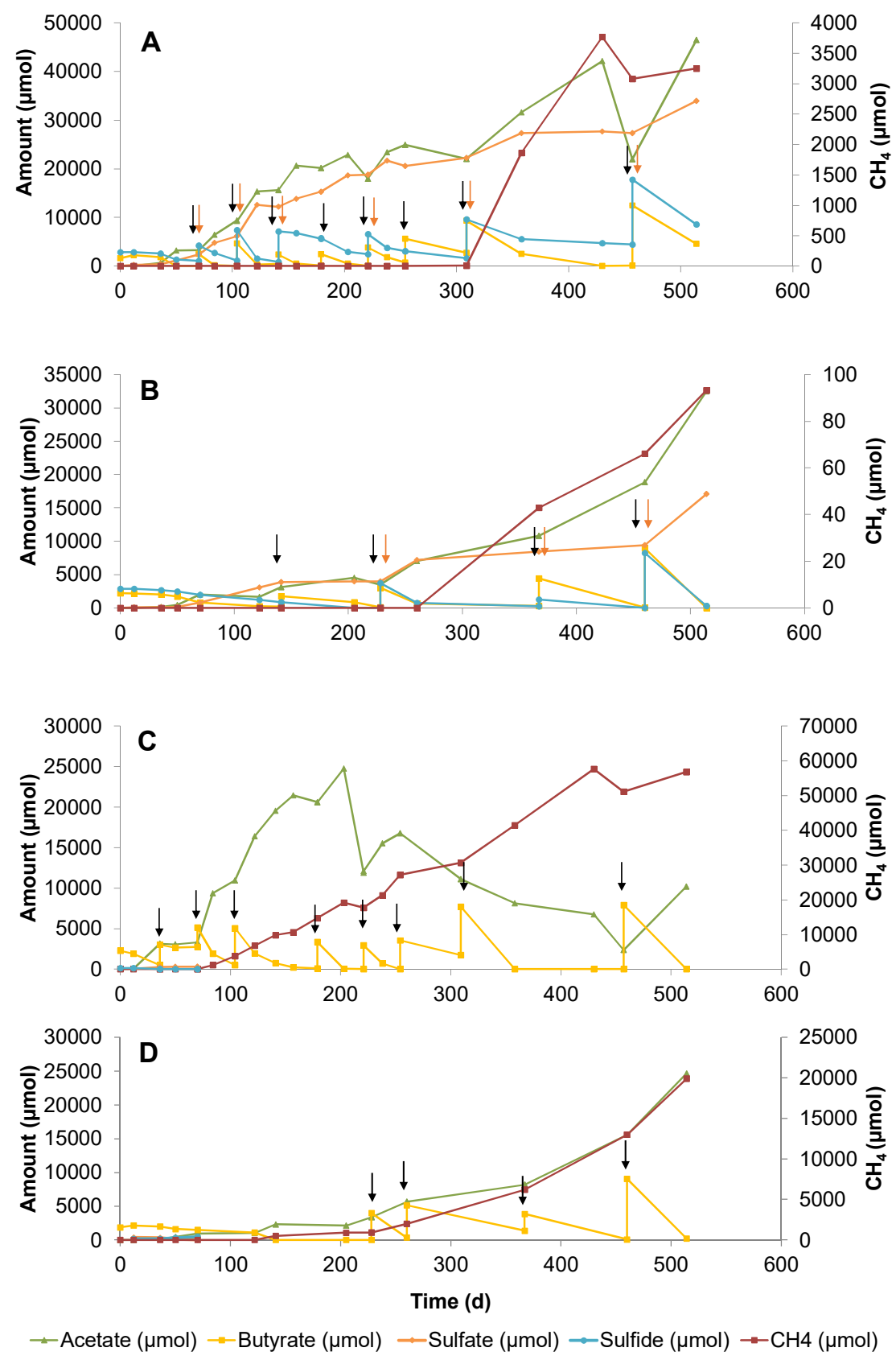

Figure 1. Changes in butyrate, sulfate, acetate, sulfide and methane concentrations during 514 days of incubation in sediment slurry enrichments constituted of sulfate zone sediment (A) Slurry B4, with sulfate addition at $25^{\circ} \mathrm{C}$, (B) Slurry $\mathrm{B} 7$, with sulfate addition at $10^{\circ} \mathrm{C}$, (C) Slurry B1, without sulfate addition at $25^{\circ} \mathrm{C}$, (D) Slurry $\mathrm{B}_{5}$, without sulfate addition at $10^{\circ} \mathrm{C}$. Arrows denote the time points for the additions of sulfate (red) and butyrate (black). 
Trace amounts of sulfate that originated from the sediment depleted within 70 days and methane formation started on day 122 . The methane concentration measured in the $10^{\circ} \mathrm{C}$ slurry at the end of the incubation period was three times less than in the $25^{\circ} \mathrm{C}$ slurry.

\subsubsection{Sulfate-methane transition zone sediment slurries}

Slurries of SMTZ sediments with low $(3 \mathrm{mM})$ and high $(20 \mathrm{mM})$ sulfate were incubated for 571 days at $25^{\circ} \mathrm{C}$ and $10^{\circ} \mathrm{C}$ (Fig 2). Butyrate was converted in high sulfate slurries similarly at both temperatures (Fig $2 \mathrm{~B}, 2 \mathrm{D}$ ). Acetate and sulfide concentrations increased by repeated butyrate and sulfate re-feeds. Methane production started on day 120 at $25^{\circ} \mathrm{C}$ (Fig $2 \mathrm{~B}$ ) and on day 229 at $10^{\circ} \mathrm{C}$ (Fig 2D) and increased slowly. The amount of methane ranged between 8 and $48 \mu \mathrm{mol} / \mathrm{bottle}$ at both temperatures until 500 days. However it rapidly reached to $4183 \mu \mathrm{mol} /$ bottle after 500 days in slurry at $25^{\circ} \mathrm{C}$.

Concerning low-sulfate containing slurries, the total butyrate concentration converted in the $25^{\circ} \mathrm{C}$ slurry was slightly higher as in the $10^{\circ} \mathrm{C}$ slurry (Fig $2 \mathrm{~A}, 2 \mathrm{C}$ ). Two times higher methane amount was measured in the $25^{\circ} \mathrm{C}$ slurry compared to the $10^{\circ} \mathrm{C}$ slurry. Acetate accumulated in the $10^{\circ} \mathrm{C}$ slurry (Fig $2 \mathrm{C}$ ), whereas no acetate was observed in the $25^{\circ} \mathrm{C}$ slurry (Fig $2 \mathrm{~A}$ ), especially after 300 days of incubation.

\subsubsection{Methanogenic zone sediment slurries}

Methanogenic zone sediment slurries were incubated for 570 days at $25^{\circ} \mathrm{C}$ and $10^{\circ} \mathrm{C}$ with and without sulfate (20mM) (Fig 3). In the $25^{\circ} \mathrm{C}$ slurries, conversion of butyrate started directly (Fig $3 \mathrm{~A}$, 3B). Repeated additions of butyrate and/or sulfate led to a steady increase in acetate and sulfide. Methane formation in the sulfidogenic slurry was observed after 90 days at $25^{\circ} \mathrm{C}$ (Fig $3 \mathrm{~A}$ ) and increased with time, whereas no methane was detected $a t 0^{\circ} \mathrm{C}\left(\mathrm{Fig}{ }_{3} \mathrm{C}\right)$ during the whole incubation period. 

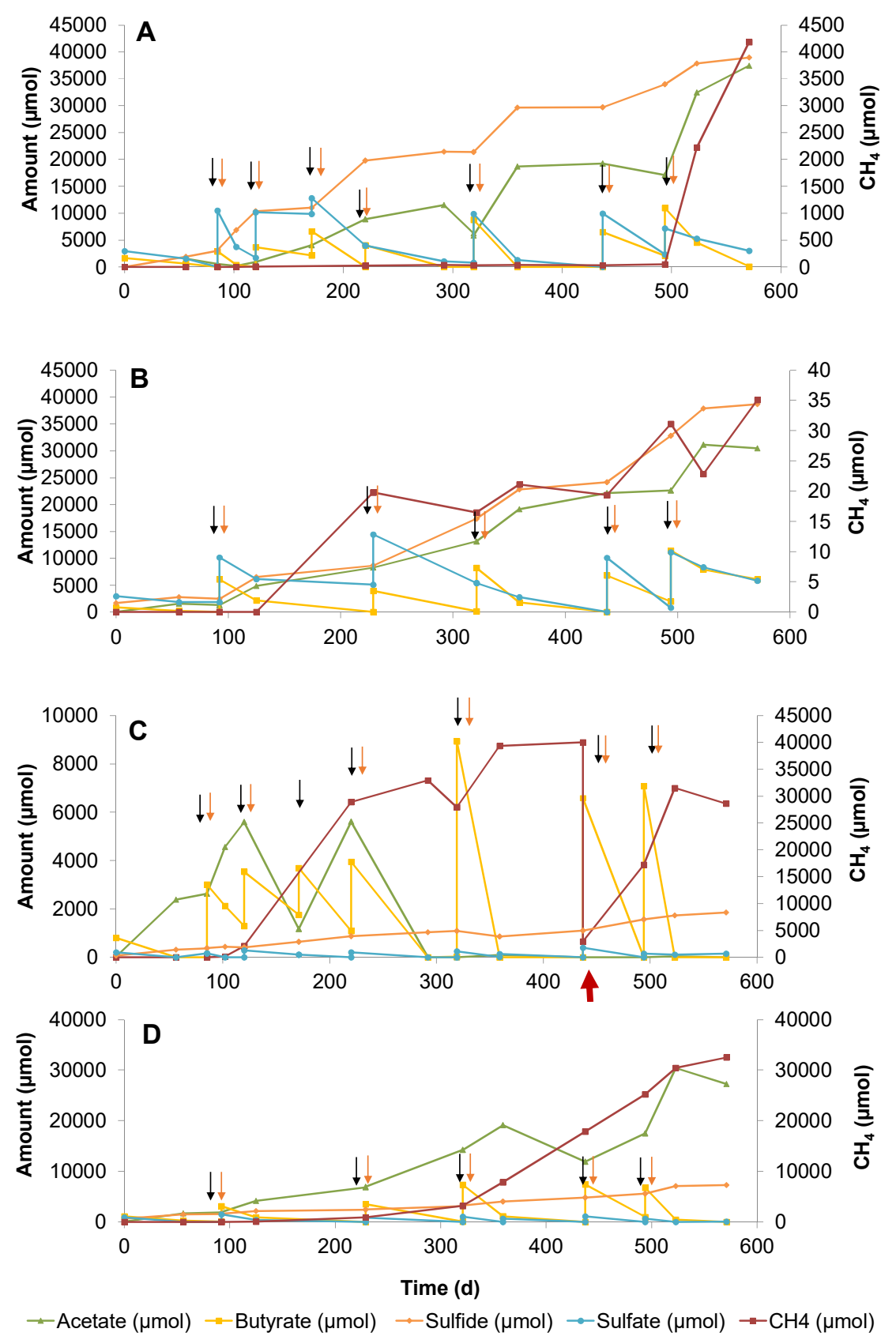

Figure 2. Changes in butyrate, sulfate, acetate, sulfide and methane concentrations during 571 days of incubation in sediment slurry enrichments constituted of sulfate-methane transition zone sediment (A) Slurry B3, with $20 \mathrm{mM}$ sulfate addition at $25^{\circ} \mathrm{C}$, (B) Slurry B7, with $20 \mathrm{mM}$ sulfate addition at $10^{\circ} \mathrm{C}$, (C) Slurry $\mathrm{B} 1$, with $3 \mathrm{mM}$ sulfate addition at $25^{\circ} \mathrm{C}$, (D) Slurry B6 with $3 \mathrm{mM}$ sulfate addition at $10^{\circ} \mathrm{C}$. Arrows denote the time points for the additions of sulfate (red) and butyrate (black). The red arrow in figure $2 \mathrm{C}$ indicates the time point that the excess amount of gas was exhausted from the headspace. 

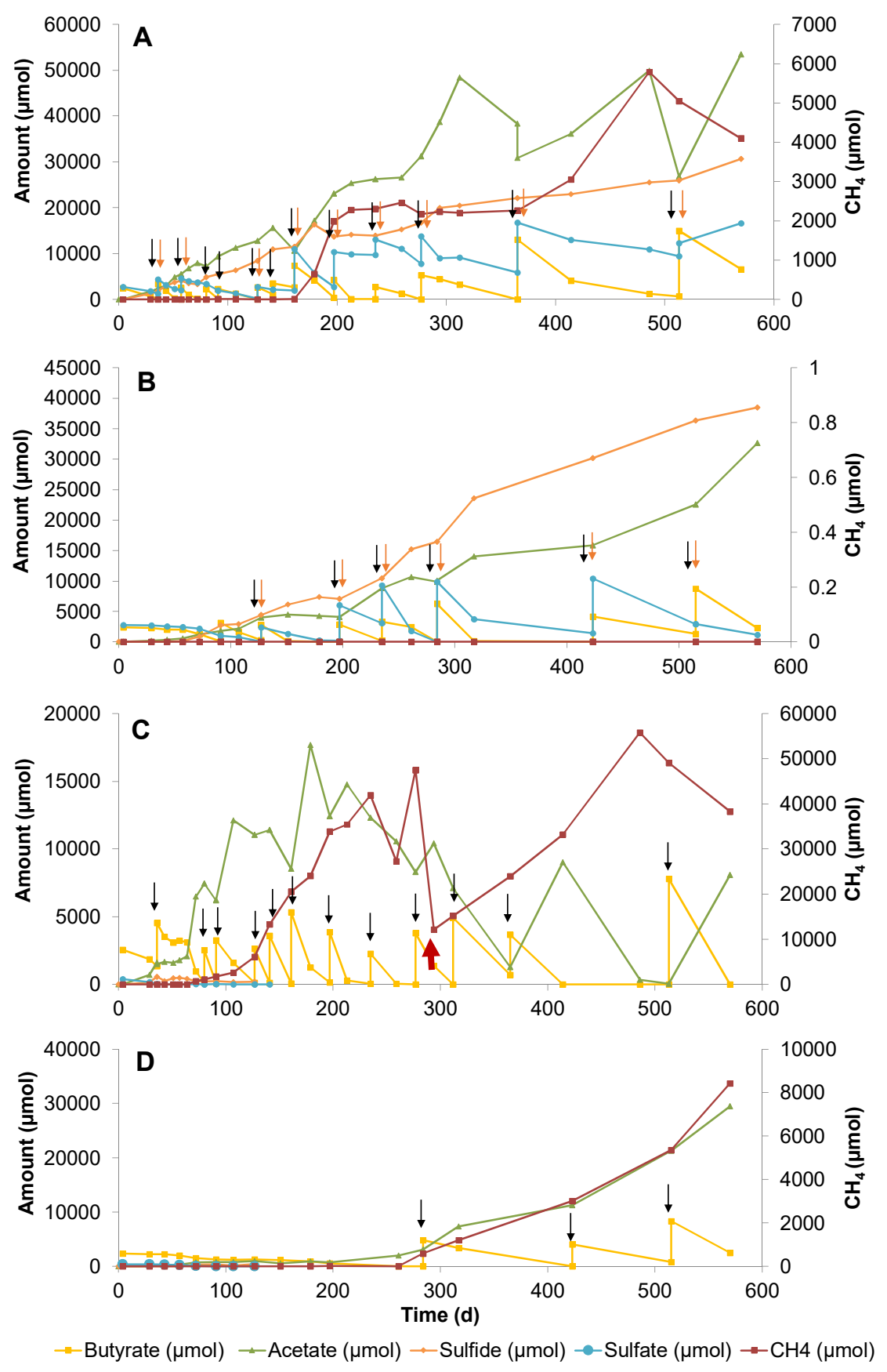

Figure 3. Changes in butyrate, sulfate, acetate, sulfide and methane concentrations during 570 days of incubation in sediment slurry enrichments constituted of methane zone sediment (A) Slurry B3, with $20 \mathrm{mM}$ sulfate addition at $25^{\circ} \mathrm{C}$, (B) Slurry B2, with $20 \mathrm{mM}$ sulfate addition at $10^{\circ} \mathrm{C}$, (C) Slurry $\mathrm{B}_{5}$, without sulfate addition at $25^{\circ} \mathrm{C}$. (D) Slurry B8, without sulfate addition at $10^{\circ} \mathrm{C}$. Arrows denote the time points for the additions of sulfate (red) and butyrate (black). The red arrow in figure ${ }_{3} \mathrm{C}$ indicates the time point that the excess amount of gas was exhausted from the headspace. 
Sulfate reduction and methanogenesis co-occurred in the sulfidogenic slurry incubated at $25^{\circ} \mathrm{C}$ (Fig $\left.3 \mathrm{~A}\right)$. The fastest butyrate conversion was observed in the methanogenic slurry at $25^{\circ} \mathrm{C}$ (Fig 3B). Trace amounts of sulfate detected in the slurry at the beginning of incubation were reduced during butyrate conversion within the first 40 days. Methane was detected at day 64 and increased rapidly due to fast conversion of butyrate. Hence, acetate and methane amounts ascended steeply within 200 days. A steady acetate consumption occurred after day 214. Even though produced after butyrate feeds, acetate was consumed again almost totally after 500 days of incubation. Since the methane pressure in this slurry reached a very high amount at day 277 , excess headspace gas was exhausted (Fig $\left.{ }_{3} \mathrm{~B}\right)$. Butyrate conversion in the methanogenic slurry at $10^{\circ} \mathrm{C}$ proceeded much slower. Methane production started at day 200 and increased gradually. In total, 11 times less methane was produced in the methanogenic slurry incubated at $10^{\circ} \mathrm{C}$ compared to the one incubated at $25^{\circ} \mathrm{C}$ within 570 days.

\subsubsection{Cluster analysis of DGGE banding pattern}

The bacterial DGGE profiles of enrichment slurries revealed that each sample taken at different time points harbored a characteristic community, illustrated by the different banding patterns. However, archaeal DGGE banding patterns of slurries were mostly similar along the incubation period except for some bands which appeared in methanogenic slurries toward the end of experiment. These bands possibly represented the dominant archaeal fractions in the enrichment slurries.

Cluster analysis of the DGGE patterns showed that all bacterial profiles corresponding to a certain sediment depth were grouped into closely related clusters and were separated from the profiles belonging to the other sediment depths (Fig S2). Each cluster representing each depth zone had several sub-clusters with less than 90\% similarity which revealed that different bacterial groups were enriched in slurries incubated under different conditions along the experiment.

Archaeal DGGE cluster analysis revealed a different clustering pattern compared to the bacterial clustering analysis (Fig S3). SZ slurry samples taken within the first 140 days of the experiment grouped together with the similarity ranging between $80 \%$ and $90 \%$. As the incubation continued, the archaeal community changed and several sub-clusters consisting of mostly two different time samples were formed, where their similarities to each other were around 70\%. SMTZ slurry samples grouped together except for the samples taken at the last sampling time indicating that the microbial communities in each slurry differentiated by the end of the experiment. MZ 
slurry samples gathered in several sub-clusters exhibiting variety in community compositions at different incubation phases. The samples belonging to the last sampling time of all MZ slurries and SMTZ $B_{3}$ and B6 slurries were grouped in the same sub-cluster, giving an indication that the enriched archaeal community in different slurries was similar.

\subsubsection{Bacterial community composition and structure}

To get insight into the microbial composition in the original sediment belonging to each zone and in slurry samples, PCR amplified partial 16S rRNA gene fragments obtained from the sediment and from the slurries at the last incubation day were sequenced. After filtering and trimming, between 3202 and 18687 high quality sequences were found per sample (Table S2) and these clustered in 62133 operational taxonomic units (OTUs; average $95 \pm 24$ ) at the family level per sample.

OTUs were classified into 33 phyla, with $96 \%$ of the OTUs belonging to 6 phyla, namely Proteobacteria (45.9\%), Chloroflexi (23.6\%), Firmicutes (17.7\%), Bacteroidetes (5.3\%), Spirochaetes (2.7\%) and Candidate division OP9 (1.1\%). Different phylotypes were abundant in different zones. Sulfate zone sediment contained Gammaproteobacteria (64\%), Chloroflexi (9\%) and Bacteroidetes (4\%) and methane zone sediment was composed of sequences belonging to Gammaproteobacteria (54\%) and Epsilonproteobacteria (27\%). On the other hand, SMTZ sediment was dominated by the sequences related to Desulfobacteraceae (79\%) belonging to the class Deltaproteobacteria (Fig S4).

Within the Proteobacteria phylum, Desulfobacteraceae was the main family that contained $61 \%$ of the reads, which is followed by Helicobacteraceae and Desulfobulbaceae having 9.4\% and $4.1 \%$ of the reads, respectively (Fig $\mathrm{S}_{4}$ ). A similar dominance of the Anaerolinaceae family with 89.2\% reads was observed in the Chloroflexi phylum. The Syntrophomonadaceae family covered $39.9 \%$ of the reads, while $50.9 \%$ of the reads belonged to uncultured Firmicutes. One of the families Desulfobacteraceae, Anaerolinaceae, Syntrophomonadaceae and uncultured Clostridiales dominated at least one slurry sample. Despite the overall dominance of these families, a high degree of variation was seen in relative abundance of them between different slurry samples.

The most abundant OTUs belonged to Desulfobacterium in the phyla Proteobacteria, uncultured Anaerolinaceae in the phylum Chloroflexi, Syntrophomonas in the phyla Firmicutes, uncultured Desulfobacteraceae in the phyla Proteobacteria, and uncultured Clostridiales in the phylum Firmicutes (Fig 4). 


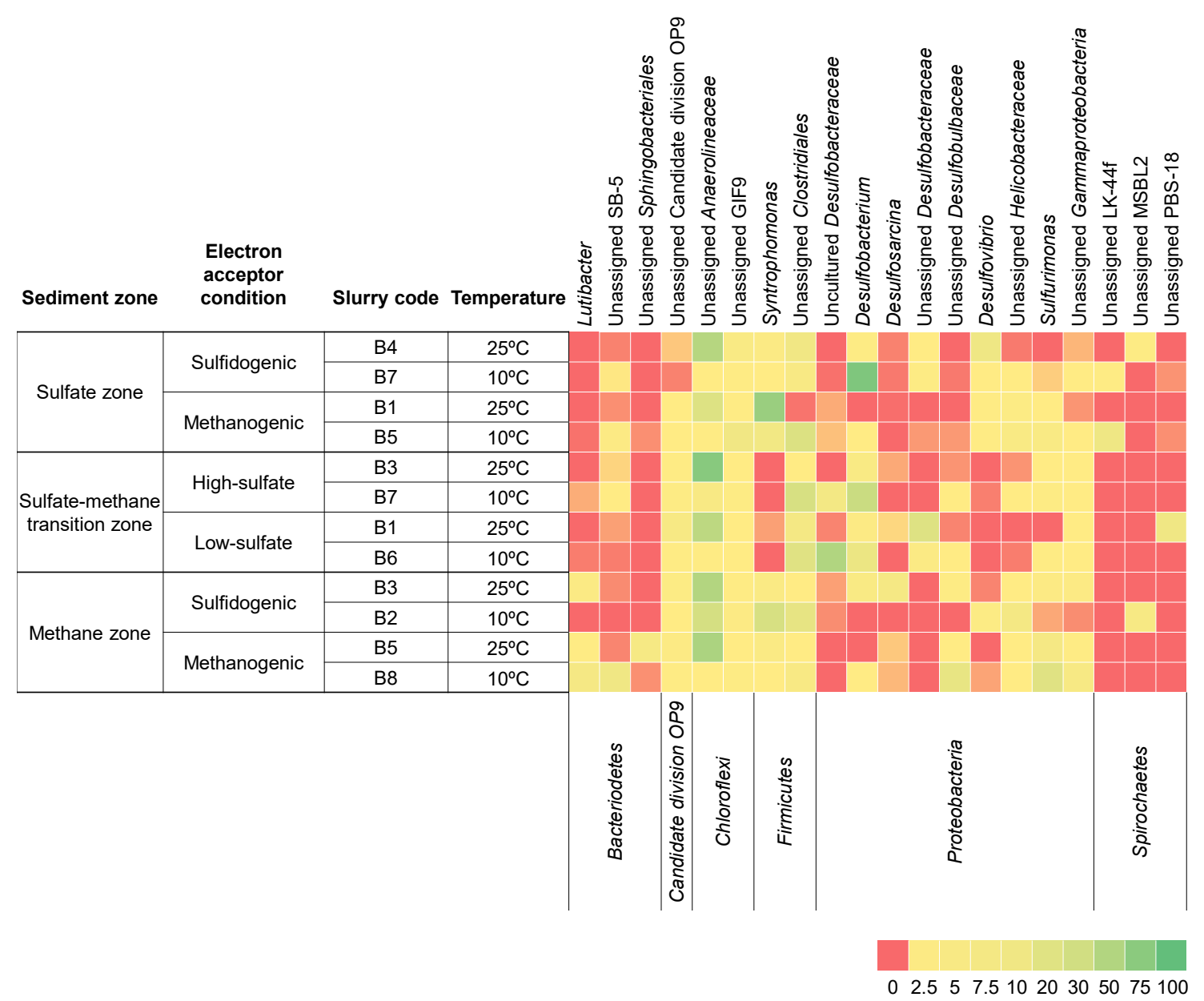

Figure 4. The heatmap depicts the relative percentage of the most common ( $>5 \%$ ) bacterial $16 \mathrm{~S}$ rRNA gene sequences across the 12 slurries analyzed. The heatmap colors represent the relative percentage of the bacterial assignments within each sample. Colors shifted towards dark green indicate higher abundance. Taxonomy is shown at the genus level (unless unassigned) above and at the phylum level below the heatmap.

Redundancy analysis separated bacterial orders by their associations to the incubation conditions (Fig 5). The order Anaerolineales (OTU 15) had the highest read abundance among all bacterial orders and showed a strong positive correlation to the temperature and butyrate (Fig 5, $\mathrm{S}_{5}$ ). Anaerolineales had positive associations with the other environmental parameters sulfate, acetate and methane. The slurries in which this order dominated were plotted on the left portion of the RDA graph. Another prevalent bacterial order Desulfobacterales (OTU 27) was observed to be associated with the slurries SZB7, SMTZB6 and SMTZB7. The order Clostridiales (OTU 22) was plotted between the slurries SZB1, SZB5 and MZB2 in which they had the highest read abundance. The slurries $\mathrm{SZB}_{5}$ and MZB8 were negatively correlated with temperature and sulfate, and was 
plotted toward the upper right portion of the RDA triplot. These slurries were associated most strongly with the orders Clostridiales (OTU 22), Campylobacterales (OTU 31) and Desulfobacterales (OTU 27) (Fig 5).

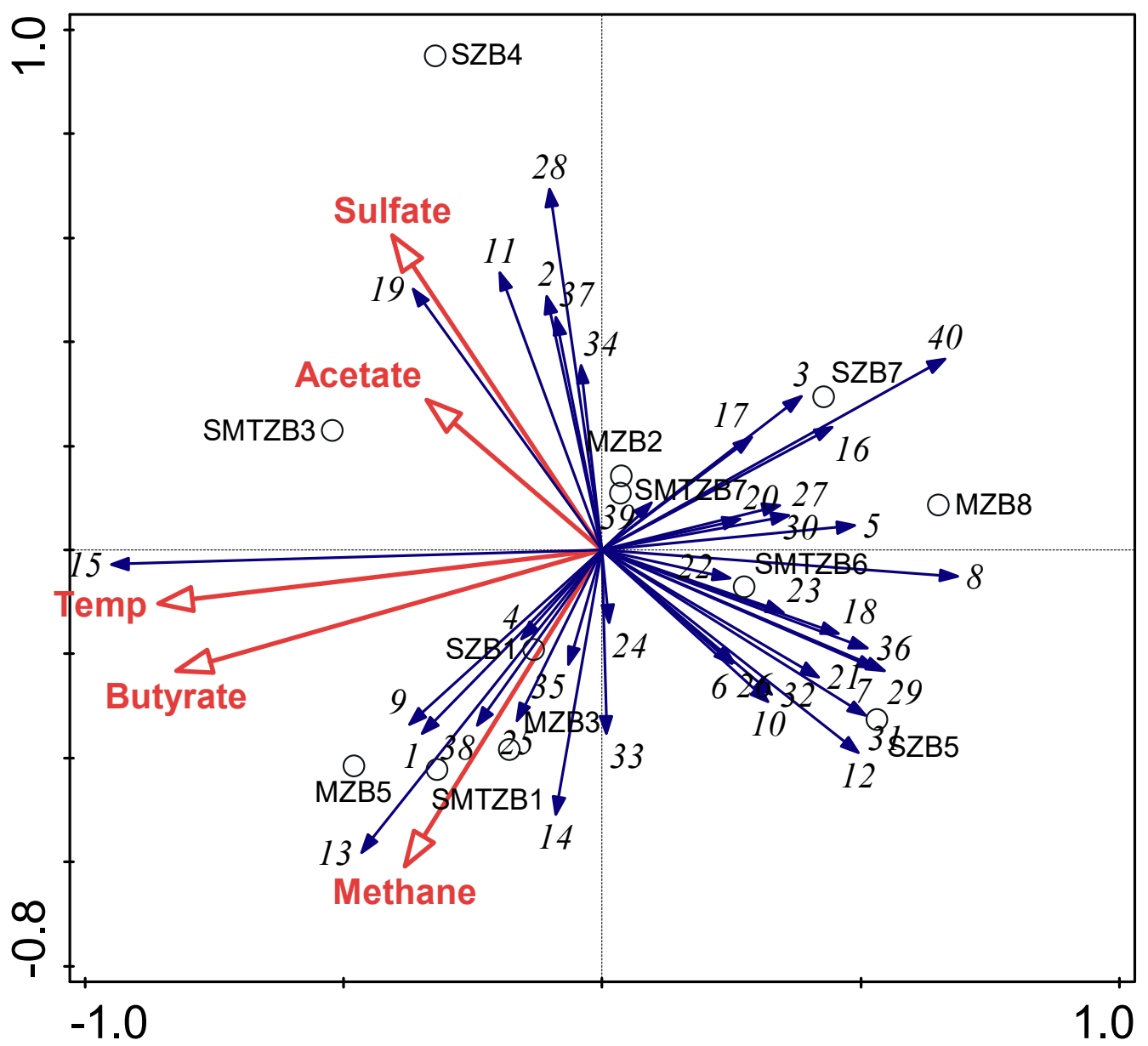

Figure 5. Redundancy Analysis Triplot showing relationship between Bacterial community composition at order level and environmental parameters. Environmental variables are given as red vectors. Blue vectors represent bacterial orders. Orders were included with a relative abundance of at least $1 \%$ in any sample. Vector length gives the variance that can be explained by a particular environmental parameter. Perpendicular distance reflects association, with smaller distances indicating a larger association. Temp: Temperature.

OTU numbers and corresponding taxa is as followed: (1)Bacteria-Other, (2)OPB41, (3)BD2-2, (4)Bacteroidales, (5)Cytophagales, (6)Flavobacteriales, (7)SB-1, (8)B-5, (9)Sphingobacteriales, (10)VC2.1.Bac22, (11)vadinHA17, (12)vadinHA17-uncultured bacterium, (13)Candidate division OP9, (14)Anaerolineae, (15)Anaerolineales, (16) GIF9, (17)MSBL5, (18)vadinBA26, (19)Chloroplast, (20)LCP-89, (21)Lineage_IV, (22)Clostridiales, (23)Nitrospirales, (24)Burkholderiales, (25)Hydrogenophilales, (26)Desulfarculales, (27)Desulfobacterales, (28)Desulfovibrionales, (29)Desulfuromonadales, (30)Sva0485, (31)Campylobacterales, (32)Gammaproteobacteria, (33)Alteromonadales, (34)Pseudomonadales, (35)Thiotrichales, (36)LK-44f, (37)MSBL2, (38)PBS-18, (39)Spirochaetaceae, (40)Acholeplasmatales. A detailed correlation matrix is provided in Table $\mathrm{S}_{5}$. 


\subsubsection{Archaeal community composition and structure}

PCR amplified partial 16S rRNA gene fragments obtained from the last day sampling of all slurries and environmental samples were sequenced. After filtering and trimming, between 9120 and 136909 high quality sequences were found per sample.

In all slurry samples, the highest average percentage of 16S rRNA reads for Archaea clustered within the families Methanomicrobiaceae (60.8\%), Methanosarcinaceae (16.3\%) and Methanosaetaeceae (9.3\%) (Fig S6). Methanomicrobiaceae dominated each slurry sample with read percentages ranging between $26 \%-98 \%$, except for one slurry incubated at $10^{\circ} \mathrm{C}$ with high sulfate in which Methanosarcinaceae became the most dominant family representing $39 \%$ of all the reads. Methanogenium belonging to the family Methanomicrobiaceae was the most dominant genus among the slurries, followed by the genus Methanosarcina from the family Methanosarcinaceae, having lower abundance (Fig 6). Methanosaetaeceae was observed only in two slurry samples incubated with low and without sulfate at $25^{\circ} \mathrm{C}$, with $43 \%$ and $67 \%$ of the reads (Fig S6). These reads belonged to Methanosaeta and unclassified Methanosaetaceae (Fig 6). The other Archaeal groups observed in different slurries were the Marine Benthic Group D/Deep Hydrothermal Vent Euryarchaeotal Group 1 (MBG-D and DHVEG-1), Miscellaneous Crenarchaeotic Group (MCG) and ANME-1b with read percentages 3.7\%, 2.5\% and 2.1\%, respectively (Fig 6, S6). 


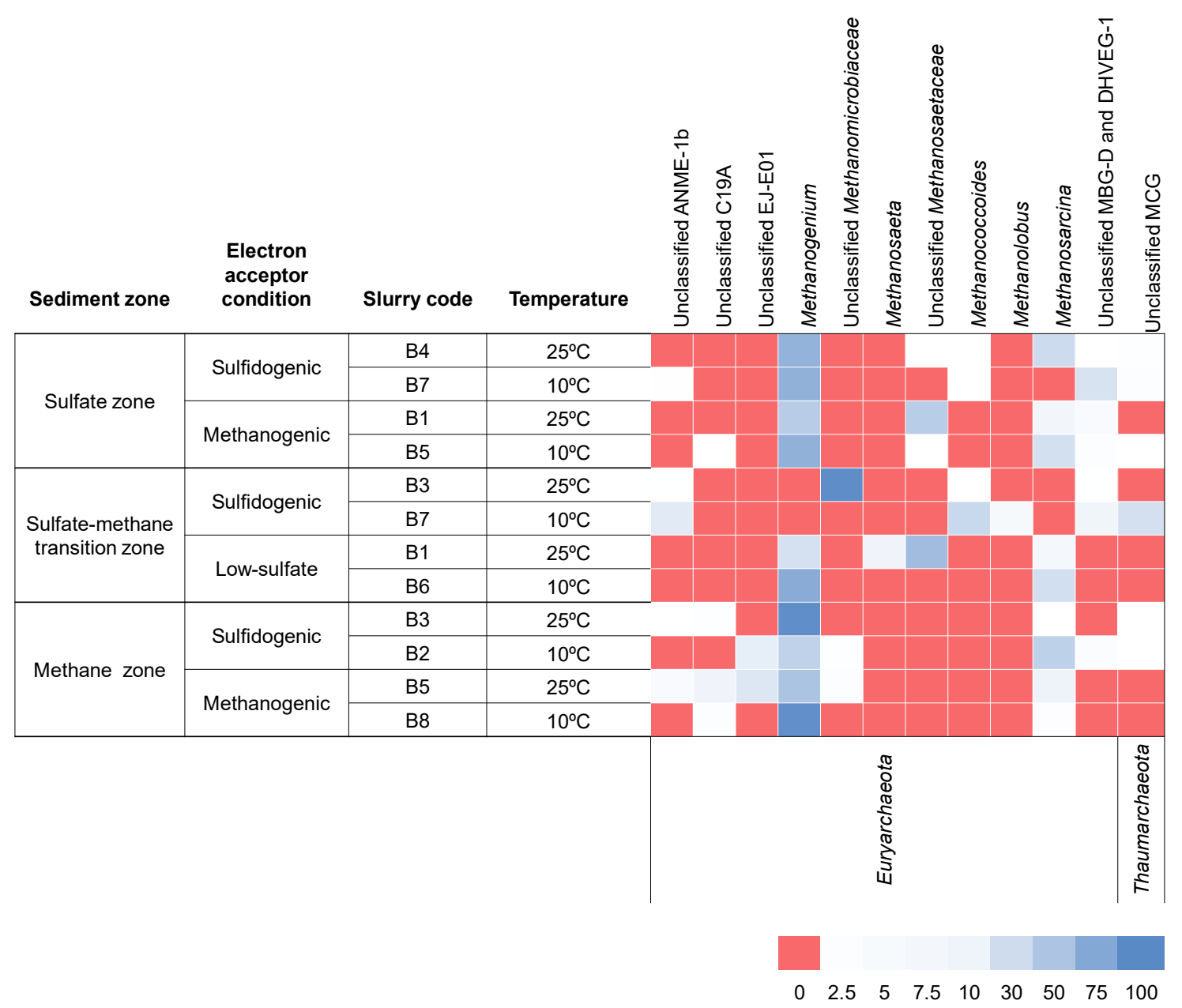

Figure 6. The heatmap depicts the relative abundance of the most common ( $>5 \%$ ) archaeal $16 \mathrm{~S}$ rRNA gene sequences (unless unclassified) across the 12 slurries analysed. The heatmap colors represent the relative percentage of the archaeal assignments within each sample. Colors shifted towards bright blue indicate higher abundance.

Archaeal community composition at family level in each slurry was plotted on RDA (Fig 7). The slurries which had high methane were plotted toward the upper left quadrant of the graph. An efficient acetate consumption was observed in these slurries, SZB1, SMTZB1 and MZB5, which was confirmed by the negative association to the acetate. Methanosaetaceae was observed to be positively correlated with temperature and negatively correlated with sulfate. The slurries $\mathrm{SZB}_{5}$, SZB4, SZB7, MZB3, MZB8, SMTZB3 in which acetate accumulated to very high concentrations showed close clustering toward the bottom middle part of the graph and were associated with acetate and the family Methanomicrobiaceae (Fig 7, Fig 1, 2, 3). The slurries that represented less Archaeal diversity and abundance were together in the bottom right portion of the RDA plot. 
Methanosarcinaceae was plotted adjacent to the slurries SMTZB6, SMTZB7 and MZB2 in which they were enriched (Fig 7, Fig 2, 3).

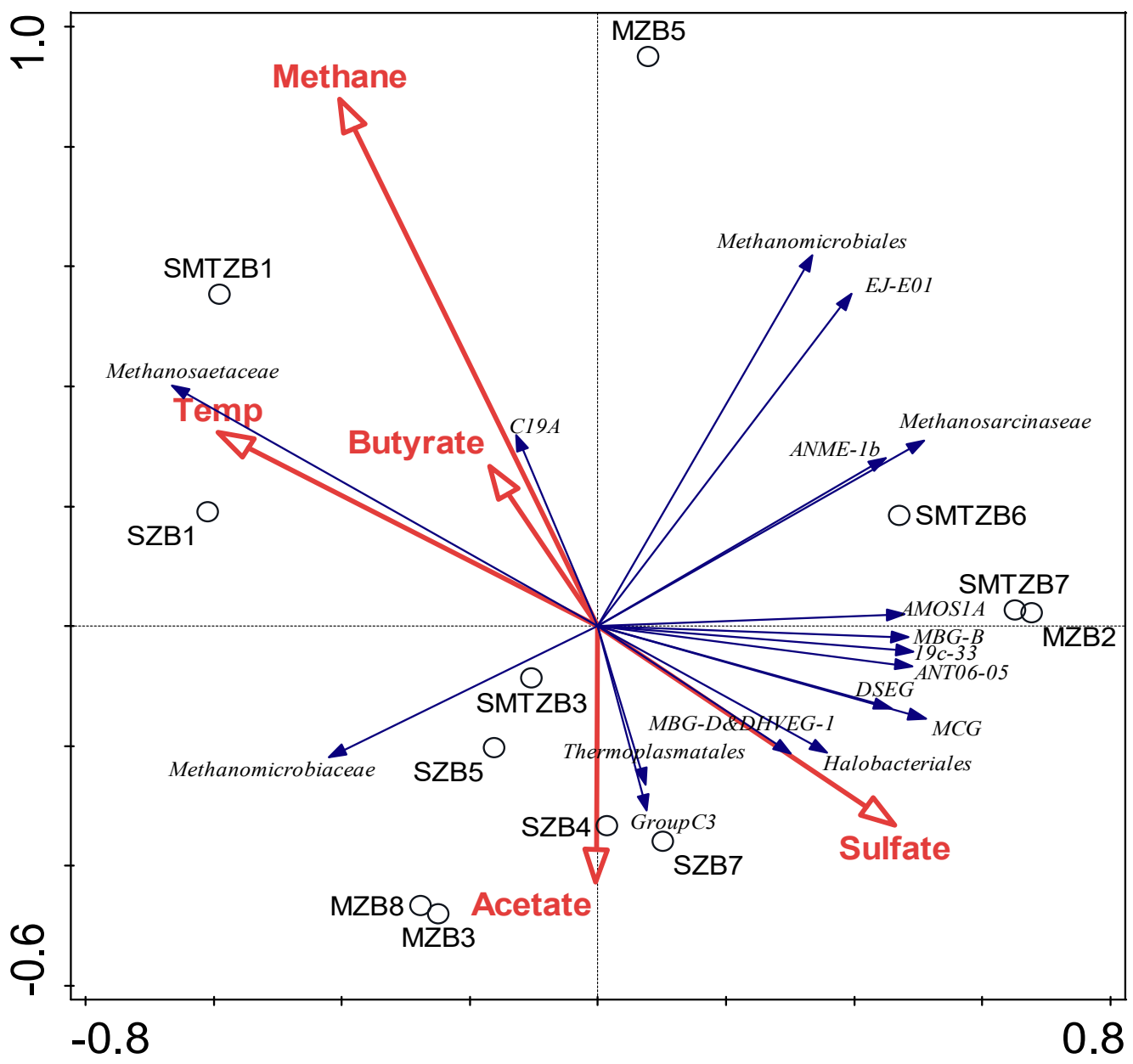

Figure 7. Redundancy Analysis Triplot showing relationship between Archaeal community composition at family level and environmental parameters. Environmental variables are given as red vectors. Blue vectors represent Archaeal families. Families were included with a relative abundance of at least $1 \%$ in any sample. Vector length gives the variance that can be explained by a particular environmental parameter. Perpendicular distance reflects association, with smaller distances indicating a larger association. Temp: Temperature.

\subsubsection{Correlation between the microbial community and between the microbial community and environmental parameters}

To investigate the relationship between the microbial community structure and incubation conditions, sequence data were correlated to environmental parameters data. Correlation analysis of the sequencing data revealed both positive and negative relations between bacterial orders (Fig $\left.\mathrm{S}_{5}\right)$. Anaerolineales, the most dominant order in the slurries, was observed to be negatively 
correlated to the other two dominant orders Desulfobacterales and Clostridiales, but positively correlated to all environmental parameters, with a strong correlation to temperature $(P<0.01)$, butyrate $(P<0.01)$ and acetate $(P<0.05)$. Desulfobacterales was negatively correlated to the orders Sphingobacteriales $(P<0.01)$, Desulfovibrionales $(P<0.05)$, Spirochaetaceae $(P<0.05)$, Clostridiales and all environmental parameters except for sulfate, and positively correlated to candidate division OP9 and Thiotrichales. Clostridiales order was observed to be positively correlated to Desulfovibrionales $(P<0.05)$, Sphingobacteriales, GIF9 belonging the phylum Chloroflexi and negatively correlated to all environmental parameters except for a weak positive correlation to sulfate. The order Campylobacterales was positively correlated to the orders Desulfuromonadales $(P<0.01)$, SB-1 $(P<0.05)$ and SB-5 $(P<0.05)$ from the phylum Bacteroidetes and unclassified Gammaproteobacteria $(P<0.05)$ and negatively correlated to Desulfobacterales, PBS-18 from the phylum Spirochaetes and to all environmental parameters except for methane.

The two aceticlastic methanogenic families Methanosaetaceae and Methanosarcinaceae did not positively correlated to each other and showed differences in their correlation to the other taxonomic groups and environmental parameters, in like manner to the difference in their absolute abundances in slurries (Fig $\mathrm{S}_{7}$ and Fig 6). Both families were negatively correlated to the hydrogenotrophic methanogenic family Methanomicrobiaceae, among which Methanosarcinaceae showed significant negative correlation $(P<0.05)$. Methanosaetaceae showed positive correlation to methane and temperature, whereas Methanosarcinaceae did not show any significant positive correlation to any of the environmental parameters. Hydrogenotrophic methanogenic families were negatively correlated with most of the other taxonomic groups. Unclassified Methanomicrobiales and EJ-Eo1 were positively correlated to methane, whereas Methanomicrobiaceae was positively correlated only to acetate.

\subsection{Discussion}

\subsubsection{Butyrate conversion in Aarhus Bay sediment}

Our results show that butyrate conversion in Aarhus Bay sediments is coupled to sulfate reduction and methane production. Previous studies indicated that sulfate reduction is an important terminal electron-accepting process in marine sediments (Jørgensen, 1982; Holmkvist et al., 2011; Bowles et al., 2014). The rapid consumption of added sulfate in sulfate-amended and sediment-originated sulfate in sulfate-free slurries suggests that sulfate reduction is the dominant pathway of butyrate 
conversion in Aarhus Bay sediment (Fig ${ }_{13} \mathrm{~B}, 2 \mathrm{~B},{ }_{3} \mathrm{~B}$ ). This is most likely due to the fact that sulfate reduction is energetically more favourable than methanogenic conversion (Muyzer and Stams, 2008). The accumulation of acetate in all slurries indicates incomplete butyrate conversion (Fig $1 \mathrm{~B}$, $2 \mathrm{~A}, 2 \mathrm{~B}, 3 \mathrm{~B})$. It is remarkable to observe methane production in the presence of sulfate in SZ slurries although late in time (Fig $1 \mathrm{~B})$. The decrease in acetate coinciding methane production suggests the occurrence of methanogenesis in the sulfate zone of Aarhus Bay, even though methanogenesis is less favourable in the presence of sulfate (Table 1). Many studies have shown the occurrence of methanogenesis in sulfate-rich marine sediments (Mori et al., 2012; Wilms et al., 2007; Beck et al., 2011; Schippers et al., 2012). Although $\mathrm{CH}_{4}$ concentrations in many sub-seafloor sediments increase only when sulfate is depleted with depth, active methanogenesis is often detected in the presence of sulfate (e.g. Woodlark Basin, Wellsbury et al., 2002 and Peru Margin, Parkes et al., 2005).

In the absence of sulfate, conversion of butyrate to acetate plus $\mathrm{H}_{2}$ is an endergonic reaction under standard conditions and becomes feasible by methanogenic partners that keep the $\mathrm{H}_{2}$ partial pressure low (Table 1) (Schink and Stams, 2013; McInerney et al., 2008). Butyrate conversion trend in sulfate-free SZ and MZ slurries incubated at $25^{\circ} \mathrm{C}$ was similar in terms of early methane production and complete acetate consumption (Fig 1A, Fig 3A). (Table 1). Apparently, the syntrophic conversion of butyrate under sulfate-free conditions is possible both in the sulfate and methane zones of Aarhus Bay sediments.

\subsubsection{The effect of sulfate concentration on conversion dynamics}

Significant differences in terms of product formation and consumption in high (20 $\mathrm{mM})$ and low (3 $\mathrm{mM}$ ) sulfate amended SMTZ slurries at $25^{\circ} \mathrm{C}$ suggests that the sulfate concentration is an important environmental factor in butyrate conversion in Aarhus Bay sediments. Low and delayed $\mathrm{CH}_{4}$ production in high sulfate amended slurries indicates possible sulfide inhibition on methanogenesis. Inhibitory effect of high sulphide concentrations on methanogens was reported previously (Pender et al., 2004; O’Flaherty et al., 1998; Shin et al., 1995). Rapid methane production after day 523 in high sulfate containing slurries might be related to the adaptation capacity of hydrogenotrophic methanogens to the slurry conditions and involvement in syntrophic butyrate conversion which caused simultaneous increase in acetate. This suggests that methanogenesis could still occur despite the on-going sulfate-reduction process and sulfide level. On the other hand, high methane production with concomitant acetate consumption in low sulfate amended SMTZ slurries indicates an efficient syntrophic butyrate conversion involving acetate- and 
hydrogen-dependent sulfate reduction and methanogenesis processes along the incubation. Apparently, low amounts of sulfate enhanced the whole microbiome, resulting in fast and dedicated population performing both sulfate reduction and methanogenesis.

\subsubsection{The effect of temperature on metabolic processes}

Different temperatures have an impact on conversion of butyrate in Aarhus Bay sediments. Lower temperatures caused lower sulfate consumption and higher acetate accumulation in SZ slurries. Similarly, below $25^{\circ} \mathrm{C}$, reduced sulfate reduction with organic acid accumulation was observed in sulfate-amended marine sediments (Weston and Joye, 2005). In contrast, the produced acetate was efficiently consumed at $25^{\circ} \mathrm{C}$ especially in sulfate-free slurries. Roussel and colleagues (2015) reported increased aceticlastic methanogenesis with increasing temperatures (from $12^{\circ} \mathrm{C}$ to $30^{\circ} \mathrm{C}$ ) in marine sediment slurries. The high-sulfate amended SMTZ slurry at $10^{\circ} \mathrm{C}$ exhibited the lowest and constantly fluctuating $\mathrm{CH}_{4}$ amounts (Fig $2 \mathrm{~B}$ ). This trend points to the existence of anaerobic oxidation of methane (AOM) in sulfate-methane transition zone of Aarhus Bay (Thomsen et al., 2001; Dale et al., 2008). Almost all the methane produced in marine sediments is consumed with concomitant sulfate removal within the SMTZ (Knittel and Boetius, 2009; Boetius et al., 200o). Therefore, despite the amounts of substrates and products did not differ significantly, low incubation temperature in SMTZ slurry favoured for a different microbial community which causes a difference in eventual metabolic process.

\subsubsection{Enriched microbial community in the presence of sulfate}

In sulfate amended slurries, enrichment of Desulfobacteraceae, Desulfovibrionaceae, Desulfobulbaceae, Syntrophomonadaceae and Clostridiales suggests that the members of these taxa are associated with butyrate conversion. Especially, Desulfobacterium, Desulfonema, Desulfosarcina, Desulfoarculus belonging to Desulfobacteraceae are known to couple butyrate degradation to sulfate reduction (Kuever et al., 2014b). The increase in the relative abundance of these genera both in the upper and the lower sediment slurries suggests the existence of sulfate reducers both in the sulfate, sulfate-methane transition and methane zones of the sediment. Leloup and colleagues (2009) showed that the sulfate-reducing bacteria in Aarhus Bay sediments are present and active within the methane zone, and have as high bacterial numbers as in the sulfate zone. Similar findings were also reported in coastal marine sediments (Limfjorden, Denmark; 
Jørgensen, 1978; Aarhus Bay, Station 6; Thomsen et al., 2001) and in deep-sea sediment (Black Sea; Leloup et al., 2007). Apparently, the ability of some SRB to grow fermentatively may explain their abundant presence in sulfate-depleted sediments and the sudden increase in their relative abundance in sulfate-amended MZ slurries. On the other hand, the increased relative abundance of Desulfovibrio in sulfate-amended slurries might be linked to the consumption of $\mathrm{H}_{2}$ produced by fermentative microorganisms. The ability of Desulfovibrio species to use $\mathrm{H}_{2}$ as electron donor and to assimilate acetate and $\mathrm{CO}_{2}$ as carbon sources supports this hypothesis (Kuever et al., 2014a). Syntrophomonas is a butyrate-degrading syntrophic specialist that grow in close association with methanogens and other hydrogen- and/or formate-using microorganisms (Plugge et al., 2011; Sousa et al., 2009; Oude Elferink et al., 1994). Sulfate-reducing bacteria that directly couple butyrate oxidation to sulfate reduction grow faster than syntrophic butyrate degraders. However, the growth rates of some syntrophic butyrate degraders were reported to be higher than some butyrateoxidizing sulfate reducers (Oude Elferink et al., 1994). In contrary, the effect of sulfate absence on the microbial community change is the increase in the relative abundance of Syntrophomonas especially in the upper sediment slurries. This result clearly shows that sulfate can decelerate the abundance of Syntrophomonas species, but does not inhibit the butyrate conversion by Syntrophomonas. On the other hand, Desulfobacteraceae and Clostridiales involved in butyrate conversion rather in SMTZ slurries. Apparently, methanogenic conditions gives Syntrophomonas a competitive advantage in the upper sediment zone over other bacteria whereas in deeper sediments niche specific or fast growing bacteria take over butyrate conversion.

\subsubsection{The effect of temperature on the enriched bacterial community}

Low temperatures have a negative effect on the relative abundance of Anaerolineaceae in the Aarhus Bay sediments. At higher temperatures $\left(25^{\circ} \mathrm{C}\right)$, Anaerolineaceae became dominant and limited the butyrate conversion due to their ability to degrade dead biomass (Kleinsteuber et al., 2012) and acetate in marine sediment slurries (Webster et al., 2011). Considering that the slurries in our study were incubated for long period, the Anaerolineaceae might have degraded the dead cells forming syntrophic relationship with hydrogenotrophic methanogens (Yamada and Sekiguchi, 2009). The higher relative abundance of Anaerolineaceae is known in the organic-rich subsurface marine sediments (Webster et al., 2004, 2006; Teske, 2006; Fry et al., 2008; Biddle et al., 2008).

Lower temperatures $\left(10^{\circ} \mathrm{C}\right)$ favours the abundance of Clostridiales. The members of the phylum Firmicutes are commonly found in surface and deep marine sediments, soils and 
methanogenic environments (Fry et al., 2008; Parkes et al., 2014). Sequences related to uncultured Firmicutes were previously found in MPN dilution cultures of hydrocarbon-contaminated sediments containing butyrate in the presence and absence of sulfate (Struchtemeyer et al., 2011). These findings points out that the Firmicutes might have played role in syntrophic butyrate conversion in $10^{\circ} \mathrm{C}$ slurries.

Although temperature did not have a significant effect on the relative abundance of Desulfobacteraceae, they were rather abundant at $10^{\circ} \mathrm{C}$. In addition, the members of Desulfobacteraceae were abundant in most of the slurries regardless of the sulfate concentration and the origin of the sediment. Pyrosequencing of $16 \mathrm{~S}$ rRNA gene amplicons retrieved from the original sediment confirmed the dominance of Desulfobacteraceae in sulfate-methane transition zone of Aarhus Bay (Fig S4). This indicates that the bacterial community at the SMTZ is distinct from other zones (Wagner et al., 2005) and this might be associated with the syntrophic interaction between Desulfobacteraceae members and methane-oxidizing archaea involving in AOM (Fig S6) (Knittel and Boetius, 2009; Boetius et al., 2000). As the incubation temperature resembles the in situ condition (Dale et al., 2008), Desulfobacteraceae members would be more successful in butyrate conversion at $10^{\circ} \mathrm{C}$ in the upper sediment slurries.

\subsubsection{Archaeal community structure in the slurries}

Methanomicrobiaceae is responsible from the consumption of $\mathrm{H}_{2} /$ formate produced by the incomplete conversion of butyrate as its members are specialists on $\mathrm{H}_{2}$ - and formate-utilization and acts as syntrophic partners (Garcia et al., 2006). In our study, Methanogenium, belonging to Methanomicrobiaceae, dominated all enrichment slurries regardless of the origin of the sediment and the incubation temperature, except for one slurry (SMTZ B7) (Fig 6, S6). This supports the finding of Garcia and colleagues (2006) considering that incomplete butyrate conversion occurred in all slurries. It is known that hydrogenotrophic methanogenesis is dominated in near-surface marine sediments (Parkes et al., 2014; Whiticar, 1999; Kendall and Boone, 2006a; Kendall et al., 2006) and that SRB and methanogenic archaea may coexist and reach equal rates of $\mathrm{H}_{2}$ consumption at high hydrogen pressures (Kristjansson et al. 1982; Parkes et al., 1990). Therefore, the absence of $\mathrm{CH}_{4}$ in sulfate-containing zones might be due to the anaerobic oxidation of methane (AOM) (Knittel and Boetius, 2009) or the adaptive mechanisms of methanogens or persistence at extremely low rates of activity even in the presence of sulfate-reducing bacteria. 
Methanosarcina is known to consume acetate and methylated compounds and some strains can reduce $\mathrm{CO}_{2}$ with $\mathrm{H}_{2}$ (Kendall and Boone, 2006b). Methanosarcina is detected in many sulfateamended and sulfate-free slurries comprised of the upper and the lower parts of the sediment in which acetate consumption occurs. Hence, Methanosarcina might be syntrophic partners of incomplete butyrate degraders and responsible for acetate consumption in those slurries. Finke et al. (2007b) suggested that acetate oxidation via interspecies $\mathrm{H}_{2}$ transfer might be a possible reason for aceticlastic methanogens to occur in sulfate-rich marine sediments. Accordingly, aceticlastic methanogens can carry out the first step of syntrophic acetate oxidation where acetate is oxidized to 2 moles of $\mathrm{CO}_{2}$ with the generation of reducing equivalents, often as hydrogen (Phelps et al., 1985). In the second step, hydrogenotrophic methanogens or sulfate reducers scavenge that hydrogen and the overall reaction becomes thermodynamically favourable. Hence, not only sulfate reducers but also the aceticlastic methanogens could grow in the sulfate zone of marine sediments (Ozuolmez et al., 2015).

The Methanosaetaceae is a specialist that uses only acetate as substrate for methanogenesis (Jetten et al., 1992). The possible reason for the predominance of Methanosaetaceae over Methanosarcinaceae in the low-sulfate SMTZ and sulfate-free SZ slurries at $25^{\circ} \mathrm{C}$ might be related to higher acetate affinity of Methanosaetaceae over Methanosarcinaceae (Jetten et al., 1992). Interestingly, the predominance of Methanosaetaceae in low-sulfate SMTZ slurry can be associated with the competition with acetate-degrading Desulfobacteraceae which is highly abundant in the original sediment (Fig S4). Although acetate is mainly consumed by sulfate reducers in marine sediments (Schönheit et al., 1982), some acetate-degrading sulfate reducers have slightly better growth kinetics than Methanosaeta (Stams et al., 2005). Similar observations were reported by Struchtemeyer et al., (2005) and speculated that low sulfate level may allow Methanosaetaceae to compete with sulfate reducers for acetate.

ANME-1 sequences have been found to be the dominant ANME type in the SMTZ of Aarhus Bay (Fig S6) (Thomsen et al., 2001; Aquilina et al., 2010; Webster et al., 2011). Timmers et al. (2015) reported that ANME-1 is more successful in low-methane, low-sulfate and high-sulfide conditions in batch incubations of Baltic Sea sediment which also resembles the characteristic of SMT zone. Low methane and high sulfide amounts in SMTZ B7 slurry supports the findings of Timmers et al. (2015) where ANME-1 was selected over ANME-2 clades under low-sulfate and high-sulfide conditions. The enrichment of both ANME-1 and Desulfobacteraceae in low temperature SMTZ slurry is in accordance with previous findings documenting that SMTZ sediments are dominated 
by the members of Desulfobacteraceae and ANME clusters, forming close associations and involving in anaerobic oxidation of methane (Fig 4, 6) (Harrison et al., 20o9; Knittel and Boetius, 2009). The other methane-oxidizing archaeal genera enriched in this slurry were Methanococcoides and Methanolobus which are defined as ANME-3 Archaea (Fig 6) (Knittel and Boetius 20o9, Lösekann et al 2007, Niemann et al 2006). Lösekann et al. (2007) reported that ANME-3 cells could live syntrophically with Desulfobulbus. Apparently, the incubation condition, inhabiting microbial community and the amounts of sulfate, sulfide and methane in the slurry favoured the enrichment of ANME-3 group.

\subsection{Conclusion}

This study demonstrates that methanogenic archaea and sulfate-reducing bacteria are present and active in sulfate, sulfate-methane transition and methane zone sediments of Aarhus Bay and there is no vertical separation of both groups in the sediment. Butyrate conversion could occur both under sulfate-reducing and methanogenic conditions regardless of the incubation temperature and the sediment depth. The conversion of butyrate by syntrophic communities throughout the sediment column suggests that continuous supply of available carbon might stimulate syntrophic butyrate degraders in the upper sulfate-rich sediment zone. On the other hand, sulfate reducers in the deeper sulfate-depleted sediment could contribute to the butyrate conversion even in the presence of low concentration of sulfate. We suggest that both groups of microbes can survive at very low activity rates in the sediment and both sulfate reduction and methanogenesis with the same substrate can proceed simultaneously. The results indicate that $\mathrm{H}_{2}$ and $\mathrm{CO}_{2}$ may be major substrates for methanogens, as $\mathrm{H}_{2}$ was never detected throughout the experiments and the members of hydrogenotrophic methanogenic family Methanomicrobiaceae were the dominant Archaea in the slurries. On the other hand, there is only limited competition between SRB and methanogens for acetate, as the aceticlastic methanogens could also be enriched in sulfatecontaining slurries. The enrichment of ANME-1 especially in SMTZ slurries together with ANME-3 suggests the possibility that these Archaea might take part in AOM interacting with other responsible microbial groups. Therefore, butyrate is converted in team work of sulfate reducers, syntrophs and methanogens throughout the anoxic marine sediment. 


\section{Acknowledgements}

We gratefully acknowledge Mark Lever (Eidgenössische Technische Hochschule, Zurich, Switzerland), Kasper Kjeldsen, Hans Røy (Aarhus University, Aarhus, Denmark) for skillful technical assistance and the captains and crews of sailing vessel Marieje and research vessel Tyra for their help during different cruises. We thank Peer Timmers and Martin Liebensteiner (Wageningen University \& Research) and Kivanc Ozuolmez for assistance during sampling and Ton van Gelder (Wageningen University\& Research) for technical assistance. This work has been funded by the Wimek Graduate School of Wageningen University \& Research and the Darwin Center for Biogeosciences (the Netherlands). Research of Alfons Stams is supported by ERC grant (project 323009) Gravitation grant (project 024.002.002) of the Netherlands Ministry of Education, Culture and Science and the Netherlands Science Foundation (NWO). 


\section{Supplementary data}

Table S1. The overview of all the enrichment slurries fed with propionate and the total amounts of the reactants consumed and products formed during the enrichment period. The enrichment slurries were consisted of sediment either from sulfate zone (SZ), sulfate-methane transition zone (SMTZ) or methane zone (MZ) and incubated at $25^{\circ} \mathrm{C}$ or $10^{\circ} \mathrm{C}$, with $3 \mathrm{mM}, 20 \mathrm{mM}$ or without (-) sulfate amendments along the study. Slurries with * were presented in the propionate conversion graphs and used for molecular analysis.

\begin{tabular}{|c|c|c|c|c|c|c|c|c|}
\hline \multirow{2}{*}{$\begin{array}{l}\text { Sediment } \\
\text { zone }\end{array}$} & \multirow{2}{*}{ Slurry Code } & \multirow{2}{*}{ Treatment } & \multirow{2}{*}{$\begin{array}{c}\text { Incubation } \\
\text { temperature }\left({ }^{\circ} \mathrm{C}\right)\end{array}$} & \multicolumn{2}{|c|}{ Reactants ( $\mu \mathrm{mol} / \mathrm{s}$ lurry) } & \multicolumn{3}{|c|}{ Products ( $\mu \mathrm{mol} / \mathrm{slurry})$} \\
\hline & & & & Butyrate & Sulfate & Acetate & Sulfide & Methane \\
\hline \multirow{8}{*}{ Sz } & B1 & - & 25 & 35085 & 313 & 37497 & 717 & 63347 \\
\hline & B2 & - & 25 & 38415 & 312 & 29386 & 508 & 64830 \\
\hline & B3 & $20 \mathrm{mM} \mathrm{SO}_{4}{ }^{2-}$ & 25 & 36896 & 37342 & 109911 & 39836 & 1030 \\
\hline & B4 & $20 \mathrm{mM} \mathrm{SO}_{4}{ }^{2-}$ & 25 & 36816 & 35382 & 74468 & 33996 & 3252 \\
\hline & B5 & - & 10 & 22092 & 351 & 24613 & 460 & 19853 \\
\hline & B6 & - & 10 & 24609 & 319 & 31487 & 364 & 12978 \\
\hline & B7 & $20 \mathrm{mM} \mathrm{SO}_{4}{ }^{2-}$ & 10 & 19587 & 15551 & 33563 & 17064 & 93 \\
\hline & B8 & $20 \mathrm{mM} \mathrm{SO}_{4}{ }^{2-}$ & 10 & 22414 & 15282 & 35718 & 18012 & 0 \\
\hline \multirow{8}{*}{ SMTZ } & *B1 & $3 \mathrm{mM} \mathrm{SO}_{4}{ }^{2-}$ & 25 & 33451 & 5500 & 10151 & 7479 & 73569 \\
\hline & B2 & $3 \mathrm{mM} \mathrm{SO}_{4}^{2-}$ & 25 & 33748 & 8020 & 30128 & 9107 & 69486 \\
\hline & *B3 & $20 \mathrm{mM} \mathrm{SO}_{4}{ }^{2-}$ & 25 & 40754 & 45716 & 45255 & 38991 & 4183 \\
\hline & B4 & $20 \mathrm{mM} \mathrm{SO}_{4}{ }^{2-}$ & 25 & 41405 & 47734 & 46877 & 42512 & 3727 \\
\hline & B5 & $3 \mathrm{mM} \mathrm{SO}_{4}^{2-}$ & 10 & 26272 & 6463 & 35148 & 7321 & 32045 \\
\hline & B6 & $3 \mathrm{mM} \mathrm{SO}_{4}^{2-}$ & 10 & 28158 & 6531 & 37574 & 7268 & 32483 \\
\hline & B7 & $20 \mathrm{mM} \mathrm{SO}_{4}^{2-}$ & 10 & 29226 & 35141 & 31129 & 36984 & 35 \\
\hline & B8 & $20 \mathrm{mM} \mathrm{SO}_{4}^{2-}$ & 10 & 33078 & 31697 & 41505 & 32413 & 1015 \\
\hline \multirow{8}{*}{ MZ } & B1 & $20 \mathrm{mM} \mathrm{SO}_{4}{ }^{2-}$ & 10 & 26059 & 18632 & 35892 & 14784 & 2956 \\
\hline & B2 & $20 \mathrm{mM} \mathrm{SO}_{4}^{2-}$ & 10 & 29488 & 34963 & 33448 & 38500 & 0 \\
\hline & B3 & $20 \mathrm{mM} \mathrm{SO}_{4}{ }^{2-}$ & 25 & 54945 & 41587 & 91742 & 30618 & 5792 \\
\hline & B4 & $20 \mathrm{mM} \mathrm{SO}_{4}{ }^{2-}$ & 25 & 56743 & 54643 & 84195 & 39675 & 587 \\
\hline & B5 & - & 25 & 45588 & 358 & 43022 & 261 & 105926 \\
\hline & B6 & - & 25 & 45829 & 782 & 29932 & 487 & 97903 \\
\hline & B7 & - & 10 & 18145 & 610 & 12727 & 380 & 27090 \\
\hline & B8 & - & 10 & 16225 & 601 & 29455 & 424 & 8417 \\
\hline
\end{tabular}


Table S2. The number of reads per sample generated by Pyrosequencing for Bacteria and HiSeq Illumina sequencing for Archaea.

\begin{tabular}{cccc}
\hline Origin & Slurry & Bacterial reads & Archaeal reads \\
\hline \multirow{4}{*}{ Sulfate zone } & ENV & 8787 & 9120 \\
& B4 & 12393 & 88555 \\
& B1 & 18687 & 121246 \\
& B7 & 12007 & 61722 \\
& B5 & 12194 & 89283 \\
\hline \multirow{5}{*}{ Sulfate-methane } & ENV & 3202 & 18696 \\
transition zone & B1 & 3758 & 75708 \\
& B6 & 5167 & 47520 \\
& B7 & 5443 & 42599 \\
& ENV & 7198 & 58464 \\
\hline \multirow{5}{*}{ Methane zone } & B3 & 10903 & 30848 \\
& B5 & 117094 & 39087 \\
& B2 & 11686 & 136909 \\
& B8 & 10891 & 52551 \\
\end{tabular}

Figure S1. Depth profiles of sediment pore water sulfate, sulfide and methane for Station M1, in Aarhus Bay, Denmark. Methane-GC1 and Methane-GC2 stands for methane concentrations retrieved from two different gravity corers, gravity corer 1 and 2, respectively. SZ; Sulfate zone, SMTZ; sulfate-methane transition zone; MZ, methane zone.






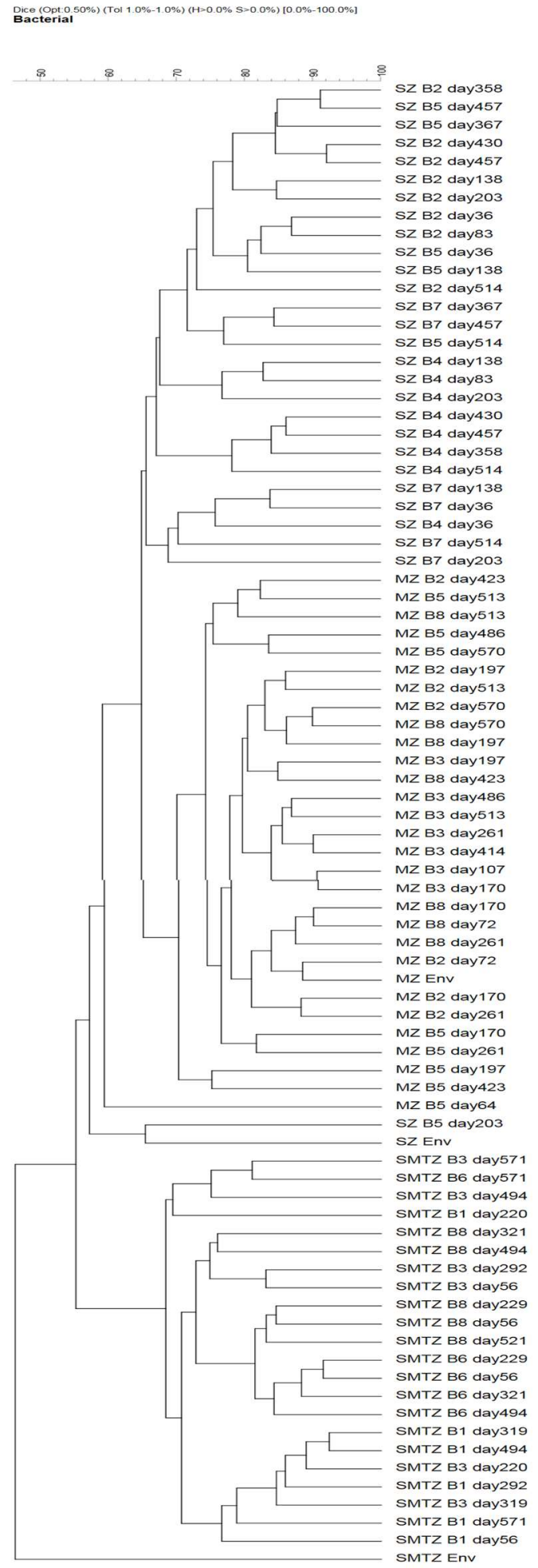

Figure S2. Cluster analysis of bacterial DGGE profiles of sediment samples taken at different biogeochemical zones and samples of enrichment slurries that were taken at different time points. The trees were generated using Dice similarity coefficient and UPGMA clustering algorithm. SZ: Sulfate zone, SMTZ: Sulfate-methane transition zone, MZ: Methane zone. 


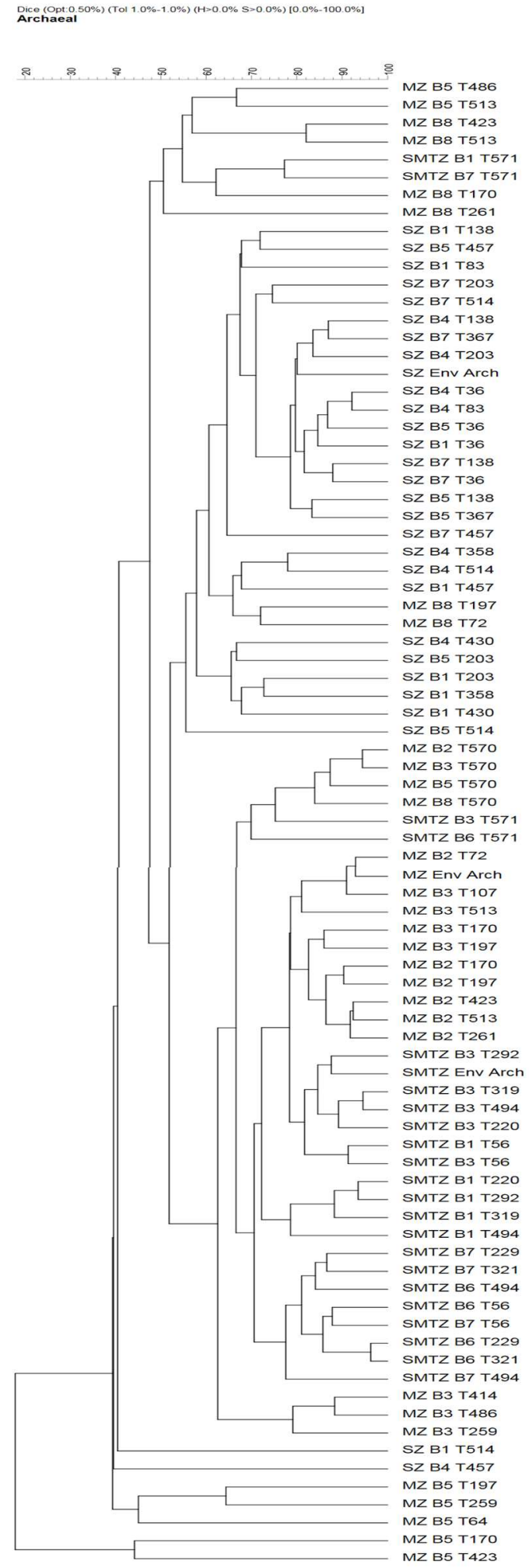

Figure S3. Cluster analysis of archaeal DGGE profiles of sediment samples taken at different biogeochemical zones and samples of enrichment slurries that were taken at different time points. The trees were generated using Dice similarity coefficient and UPGMA clustering algorithm. SZ: Sulfate zone, SMTZ: Sulfate-methane transition zone, MZ: Methane zone. 
Figure S4. Relative abundances of the bacterial community in all slurries and environmental samples at family level, normalized to $100 \%$. Only those families that were present at an abundance $>1 \%$ in at least one sample were included in the graph. SZ: Sulfate zone, SMTZ: Sulfate-methane transition zone; MZ: Methane zone, Env: Sediment sample belonging to the indicated biogeochemical zone. S: $20 \mathrm{mM}$ sulfate, $3 \mathrm{~S}: 3 \mathrm{mM}$ sulfate is used as electron acceptor in slurries. Slurries that were not labeled with 'S' or ' $3 \mathrm{~S}^{\prime}$ ' were incubated without sulfate. The number of reads of each sample was given in brackets.

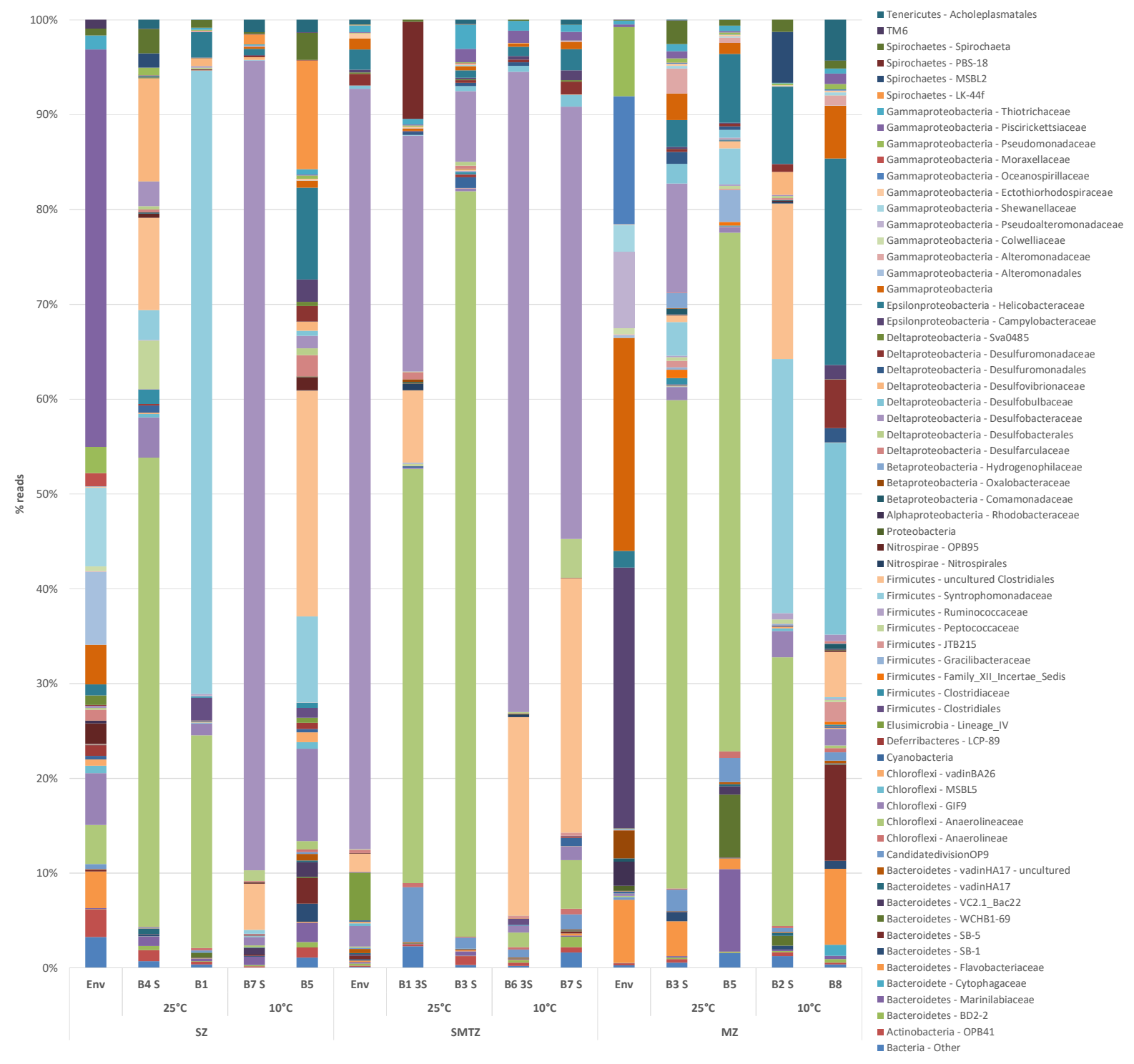


Figure S5. The heatmap depicts the correlation between bacterial orders present at a relative abundance $>1 \%$ of total reads across the 12 slurry samples and experimental parameters. Correlations were determined by means of the two tailed Spearman's Rank Order Correlation test. The heatmap colors represent the relative percentage of the microbial order assignments. Square colors shifted towards bright green indicate strong correlation.

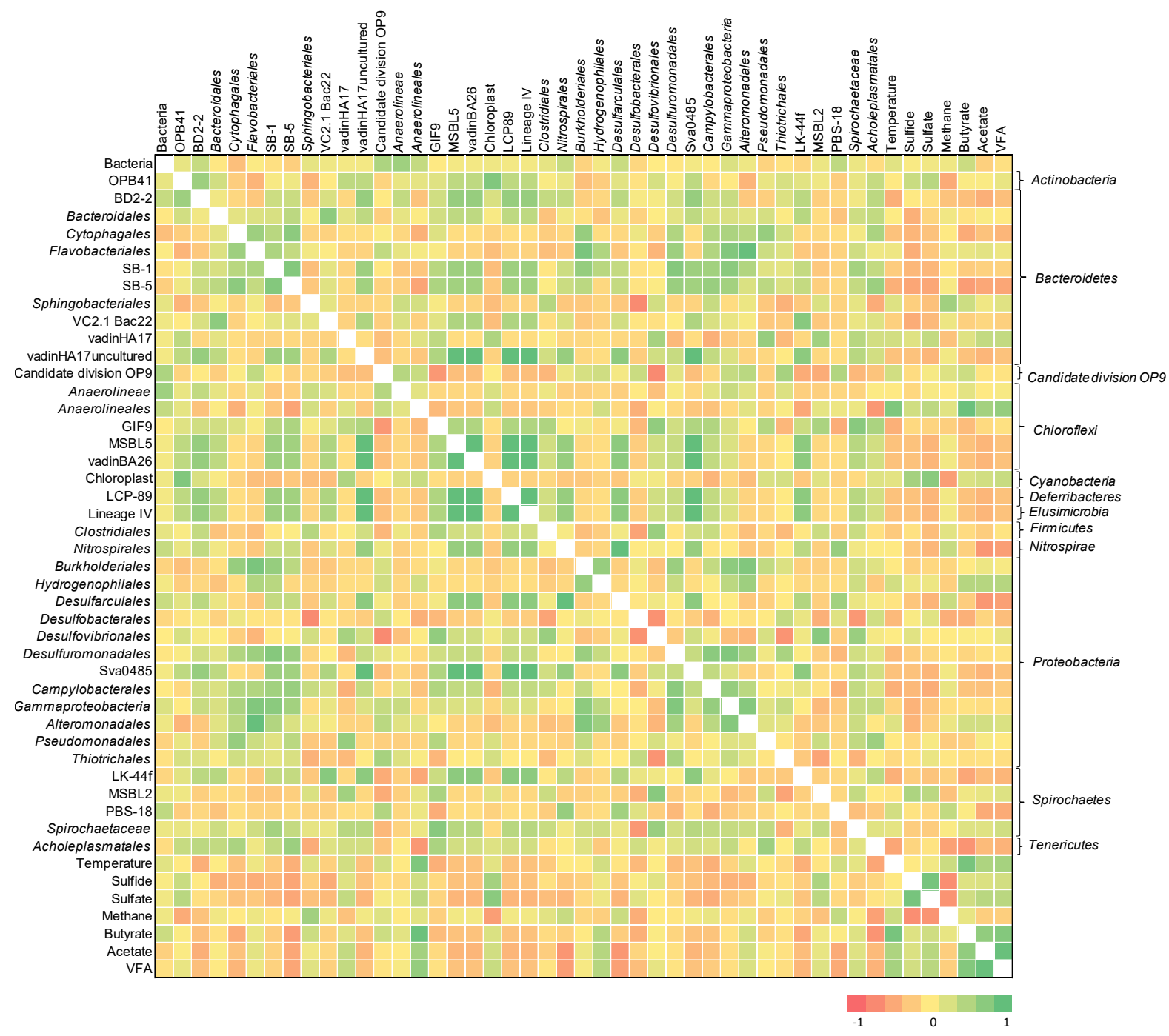


Figure S6. Relative abundances of the archaeal community in all slurries and environmental samples at family level, normalized to $100 \%$. Only those families that were present at an abundance $>1 \%$ in at least one sample were included in the graph. SZ: Sulfate zone, SMTZ: Sulfate-methane transition zone; MZ: Methane zone. Env: Sediment sample belonging to the indicated biogeochemical zone. $\mathrm{S}$ : $20 \mathrm{mM}$ sulfate, ${ }_{3} \mathrm{~S}: 3 \mathrm{mM}$ sulfate is used as electron acceptor in slurries. Slurries that were not labeled with 'S' or ' $3 S^{\prime}$ ' were incubated without sulfate.

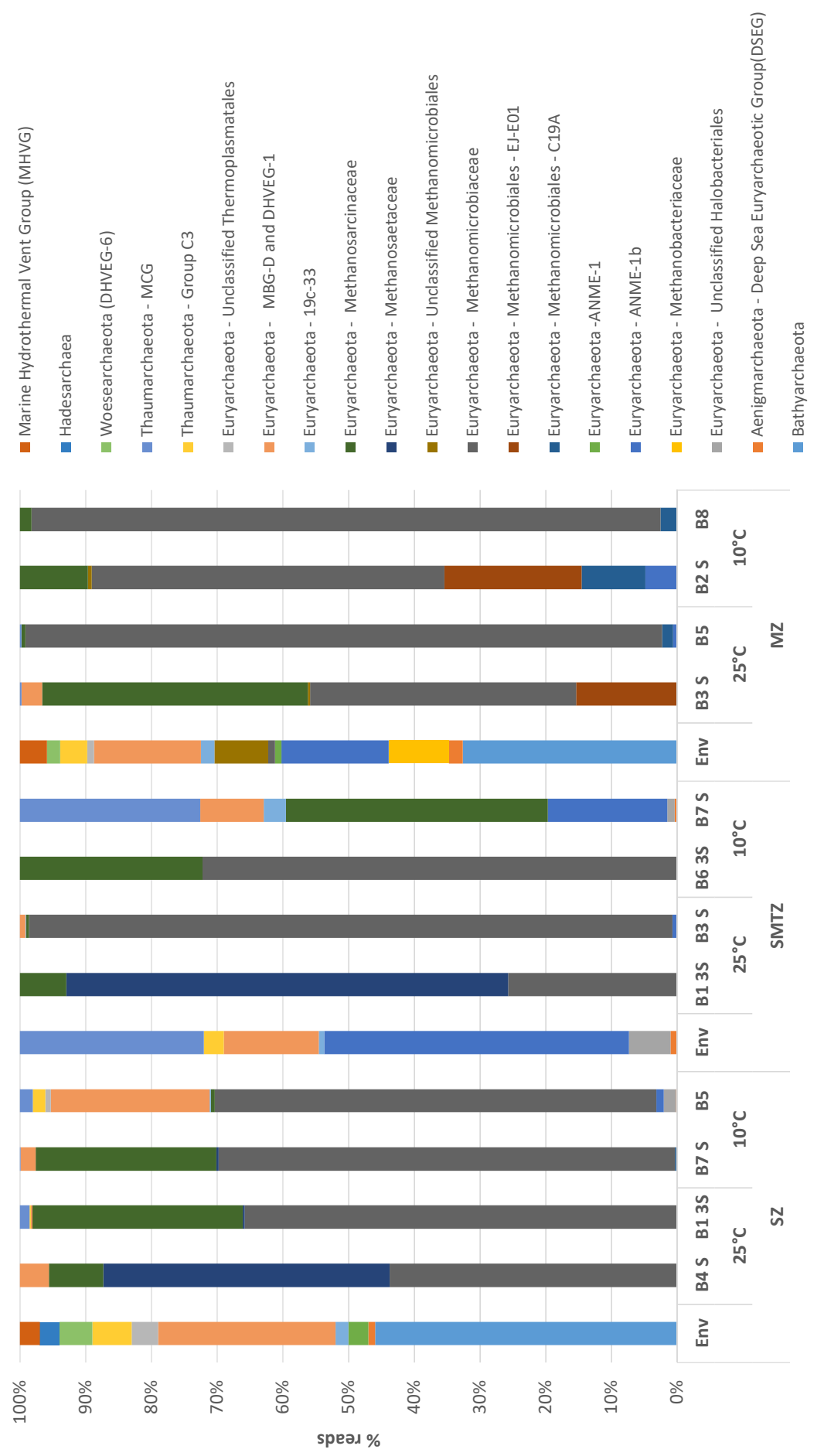


Figure S7. The heatmap depicts the correlation between archaeal families present at a relative abundance $>\mathbf{1} \%$ of total reads across the 12 slurry samples analyzed and experimental parameters. Correlations were determined by means of the two tailed Spearman's Rank Order Correlation test. The heatmap colors represent the relative percentage of the microbial family assignments. Square colors shifted towards bright green indicate strong correlation.

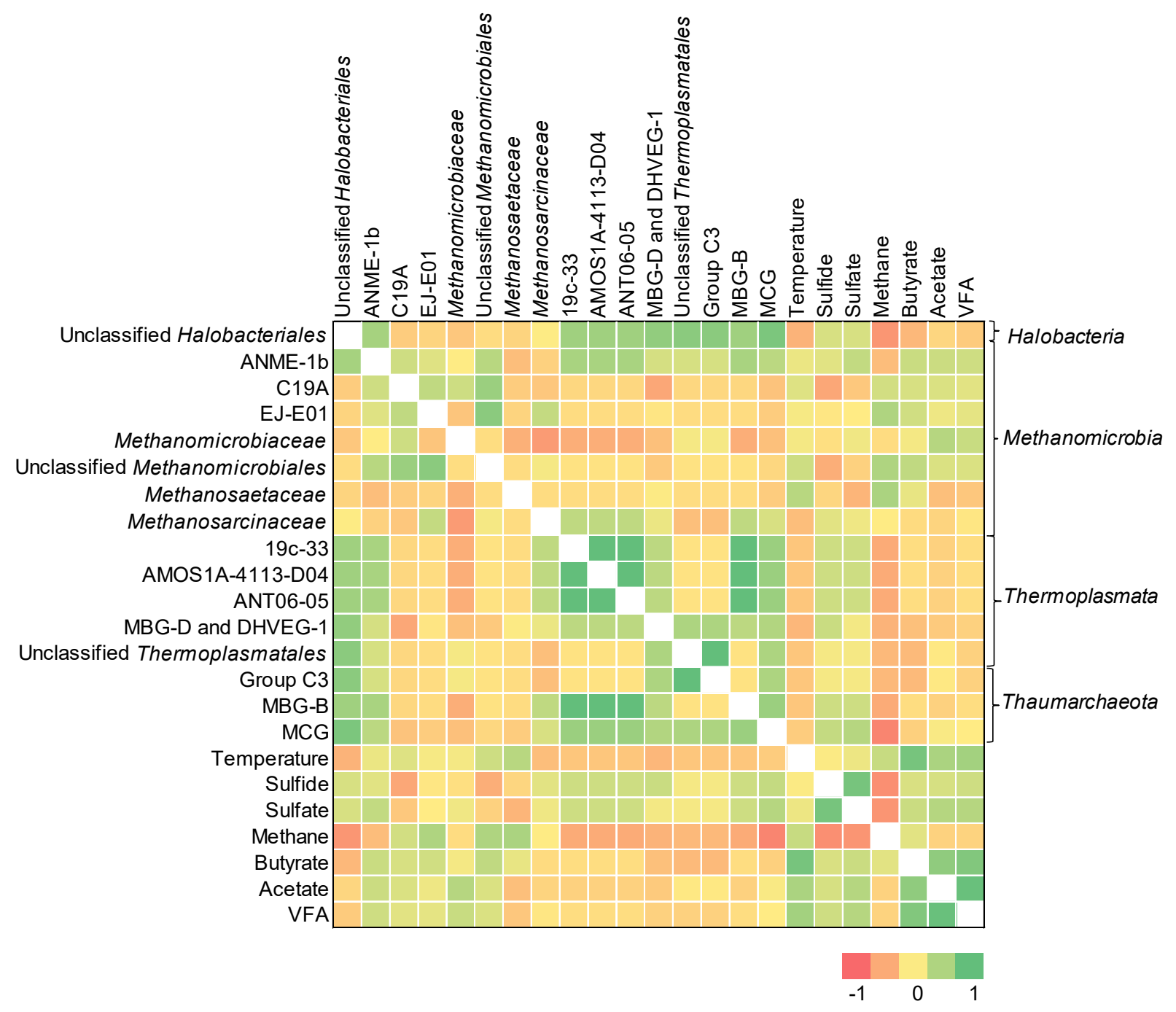




\section{Chapter 4}

\section{Propionate conversion under sulfidogenic and methanogenic conditions in different biogeochemical zones of Aarhus Bay, Denmark}




\section{Abstract}

Propionate conversion was analysed in enrichment slurries containing sediment from different biogeochemical zones of Aarhus Bay, Denmark, and the enriched prokaryotic community was determined at the end of the incubation period. Sediment slurries were amended with 3, $20 \mathrm{mM}$ sulfate and without sulfate at $25^{\circ} \mathrm{C}$ and $10^{\circ} \mathrm{C}$. After $514-571$ days of incubation, methanogenesis in the sulfate zone and sulfate reduction in the methane zone slurries was observed and both processes occurred simultaneously through the whole sediment. Bacterial community analysis revealed the dominance of Desulfobacteraceae and Desulfobulbaceae members in sulfidogenic slurries incubated both at $25^{\circ} \mathrm{C}$ and $10^{\circ} \mathrm{C}$. Sulfate-free slurries at $25^{\circ} \mathrm{C}$ were dominated with the sequences related to Cryptanaerobacter belonging to Peptococcaceae. Sulfate-free slurries incubated at $10^{\circ} \mathrm{C}$ consisted of sequences related to Desulfobacteraceae. Archaeal community analysis revealed the prevalence of different genera of the Methanomicrobiales in slurries incubated at different temperatures and with different sulfate concentrations, and the occurrence of Methanosarcinaceae was only observed in the absence of sulfate. In summary, our results show that Aarhus Bay sediment contains sulfate reducers, syntrophs and methanogens throughout the sediment, suggesting teamwork in the conversion of propionate. 


\subsection{Introduction}

The mineralization of organic matter in anoxic marine sediments is a sequential process, in which several intermediates are produced by fermentative bacteria. These intermediates, including acetate, propionate, butyrate, are eventually degraded to carbon dioxide and/or methane. Sulfate reduction and methanogenesis are important terminal electron-accepting processes and controlled mainly by the amount of available sulfate (Jørgensen, 1982; Reeburgh and Heggie, 1977; Winfrey and Zeikus, 1977). In marine sediments, sulfate reduction is considered to be dominant over methanogenesis as the primary terminal electron accepting step in the degradation of organic matter (Kristjansson et al., 1982; Lovley et al., 1982). Propionate is one of the important intermediates in anaerobic degradation process and can be oxidized by several marine sulfatereducing bacteria either completely to carbon dioxide or incompletely to acetate (Table 1). These SRB belong to the families Desulfobacteraceae, Desulfobulbaceae, Syntrophobacteraceae and Peptococcaceae (Rabus et al., 2013; Kuever, 2014b, 2014c; Leloup et al., 2007 and 2009). Incompleteoxidizing sulfate reducers, such as Desulfobulbus spp., convert propionate to acetate and carbon dioxide (Kuever, 2014c). The end-product acetate is subsequently consumed by other sulfate reducers such as Desulfobacter spp. in the presence of sulfate.

In the absence of sulfate, complete propionate conversion to $\mathrm{CH}_{4}$ takes place and is only possible by cooperation of syntrophic bacteria with methanogens (Schink and Stams, 2013; Stams, 1994) (Table 1). Syntrophic fatty acid metabolism is the rate limiting step of organic carbon degradation, but contributes to the carbon flux significantly in methanogenic environments (Schink, 1997; Schink and Stams, 2013). Despite previous studies, claiming that sulfate reduction and methanogenesis are temporally or spatially separated depending on sulfate concentration (Cappenberg, 1974; Mountfort and Asher, 1979), both processes co-occur in anoxic marine environments in the presence of high organic carbon (Oremland and Taylor, 1978; Oremland et al., 1982; Senior et al., 1982). Therefore, an excessive amount of available organic carbon may lead methanogenic archaea inhabit the habitats rich in sulfate and contribute to organic matter degradation together with syntrophic bacteria.

The relative distribution of sulfate-reducing bacteria in Black Sea and Aarhus Bay sediments shows that SRB are present in the methane zone with a similar high abundance as in the sulfate zone (Leloup et al., 2007 and 2009). It is speculated that SRB and methanogens cooperate for the mineralization of organic matter in Black Sea and Aarhus Bay sediments. Similarly, Kendall and colleagues (2006) described marine propionate- and butyrate-degrading syntrophs and suggested 
that syntrophic associations have a great role in the methane reserves in marine sediments. Krylova and Conrad (1998) demonstrated that there are yet uncultured propionate-converting bacteria which act either as SRB or syntrophs depending on sulfate availability. Therefore, it is crucial to understand how sulfate-reducers, syntrophs and methanogens interact during propionate conversion under high and low sulfate conditions in marine sediments.

Table 1. Overview of reactions examined in this study. $\Delta \mathrm{G}$ values were obtained from Thauer et al., 1977.

\begin{tabular}{|c|c|c|c|c|}
\hline \multirow[t]{2}{*}{ No. } & \multicolumn{3}{|l|}{ Equation } & \multirow[t]{2}{*}{$\Delta \mathrm{G}^{\circ 1}$ (kJ/reaction)* } \\
\hline & Acetogenic reactions & & & \\
\hline \multirow[t]{2}{*}{1} & Propionate $^{-}+3 \mathrm{H}_{2} \mathrm{O}$ & $\rightarrow$ & Acetate $^{-}+\mathrm{HCO}_{3}^{-}+3 \mathrm{H}_{2}+\mathrm{H}^{+}$ & +76.1 \\
\hline & Sulfate-reducing reaction & & & \\
\hline 2 & Propionate ${ }^{-}+0.75 \mathrm{SO}_{4}^{2-}$ & $\rightarrow$ & Acetate ${ }^{-}+0.75 \mathrm{HS}^{-}+\mathrm{HCO}_{3}^{-}+0.25 \mathrm{H}^{+}$ & -37.8 \\
\hline 3 & $4 \mathrm{H}_{2}+\mathrm{SO}_{4}^{2-}+\mathrm{H}^{+}$ & $\rightarrow$ & $\mathrm{HS}^{-}+4 \mathrm{H}_{2} \mathrm{O}$ & -151.9 \\
\hline \multirow[t]{2}{*}{4} & Acetate ${ }^{-}+\mathrm{SO}_{4}{ }^{2-}$ & $\rightarrow$ & $2 \mathrm{HCO}_{3}^{-}+\mathrm{HS}^{-}$ & -47.6 \\
\hline & Methanogenic reactions & & & \\
\hline 5 & $4 \mathrm{H}_{2}+\mathrm{HCO}_{3}^{-}+\mathrm{H}^{+}$ & $\rightarrow$ & $\mathrm{CH}_{4}+3 \mathrm{H}_{2} \mathrm{O}$ & -135.6 \\
\hline \multirow[t]{2}{*}{6} & Acetate $^{-}+\mathrm{H}_{2} \mathrm{O}$ & $\rightarrow$ & $\mathrm{CH}_{4}+\mathrm{HCO}_{3}^{-}$ & -31.0 \\
\hline & Syntrophic propionate co & & & \\
\hline \multirow[t]{2}{*}{$1+5$} & Propionate ${ }^{-}+0.75 \mathrm{H}_{2} \mathrm{O}$ & $\rightarrow$ & Acetate ${ }^{-}+0.75 \mathrm{CH}_{4}+0.25 \mathrm{HCO}_{3}^{-}+0.25 \mathrm{H}^{+}$ & -25.6 \\
\hline & Complete propionate con & & & \\
\hline \multirow[t]{2}{*}{$2+4$} & Propionate ${ }^{-}+1.75 \mathrm{SO}_{4}^{2-}$ & $\rightarrow$ & $1.75 \mathrm{HS}^{-}+3 \mathrm{HCO}_{3}^{-}+0.25 \mathrm{H}^{+}$ & -85.4 \\
\hline & Complete propionate con & netl & nogens & \\
\hline $1+5+6$ & Propionate $-1.75 \mathrm{H}_{2} \mathrm{O}$ & $\rightarrow$ & $1.75 \mathrm{CH}_{4}+1.25 \mathrm{HCO}_{3}^{-}+0.25 \mathrm{H}^{+}$ & -56.6 \\
\hline
\end{tabular}

In this study, we aimed to identify the propionate conversion process and the responsible microorganisms in the sulfate, sulfate-methane transition and methane zones of Aarhus Bay, Denmark. We set up batch slurries with and without sulfate additions and incubated them at $10^{\circ} \mathrm{C}$ and $25^{\circ} \mathrm{C}$. In this way, we examined the effect of sulfate concentration, sediment depth and temperature on the ultimate enriched bacterial and archaeal community.

\subsection{Materials and methods}

The materials and methods section of this chapter is exactly the same as the materials and methods section of the chapter 3. Therefore, please consult Chapter 3. 


\subsection{Results}

\subsubsection{Sampling site geochemistry}

The geochemistry of the Aarhus Bay sediment was revealed by pore water analysis and biogeochemical zones were defined as sulfate zone between $\mathrm{o} \mathrm{cm}$ and $120 \mathrm{~cm}$, sulfate-methane transition zone (SMTZ) between $120 \mathrm{~cm}$ and $170 \mathrm{~cm}$ and methane zone between $170 \mathrm{~cm}$ and $300 \mathrm{~cm}$ (Fig S1).

\subsubsection{Sediment slurry enrichments}

\subsubsection{Sulfate zone sediment slurries}

Sulfate zone sediment slurries were incubated for 514 days at $25^{\circ} \mathrm{C}$ and $10^{\circ} \mathrm{C}$. In sulfate-amended slurries, propionate conversion started immediately. Acetate and sulfide steadily increased as a result of repeated additions of propionate and sulfate. Slight changes were detected in acetate concentration between days 220 and 430 at $25^{\circ} \mathrm{C}$, which is followed by a decrease. After propionate addition, acetate concentration increased again in response to propionate conversion (Fig $1 \mathrm{~A}$ ). In contrast, propionate conversion in $10^{\circ} \mathrm{C}$ slurries started after 50 days of incubation and was slower along the incubation period (Fig ${ }_{1 B}$ ). Several additions of propionate and sulfate yielded acetate and sulfide. Methane formation in sulfidogenic slurries was observed after 309 days at $25^{\circ} \mathrm{C}$ (Fig $1 \mathrm{~A}$ ), while no methane was detected in slurries incubated at $10^{\circ} \mathrm{C}$ (Fig $\left.1 \mathrm{~B}\right)$ throughout the study.

In sulfate-free sediment slurries, $\sim 1 \mathrm{mM}$ sulfate was detected at the beginning of the incubation, which originated from the sediment $\left(\mathrm{Fig}_{1} \mathrm{C},{ }_{1} \mathrm{D}\right)$. In the first 50 days of incubation at $25^{\circ} \mathrm{C}$, conversion of propionate was coupled to sulfate reduction ( $\mathrm{Fig}_{1} \mathrm{C}$ ). Several feeds of propionate led to the formation of methane and acetate until day 238 . After that day, the acetate concentration fluctuated. Propionate conversion at $10^{\circ} \mathrm{C}$ was slow. Trace amounts of sulfate were depleted in 70 days and methane formation started after 260 days of incubation (Fig $1 \mathrm{D}$ ). The total methane produced in $10^{\circ} \mathrm{C}$ slurries was half the amount of methane that was produced in $25^{\circ} \mathrm{C}$ slurries in the end of the experiment. 

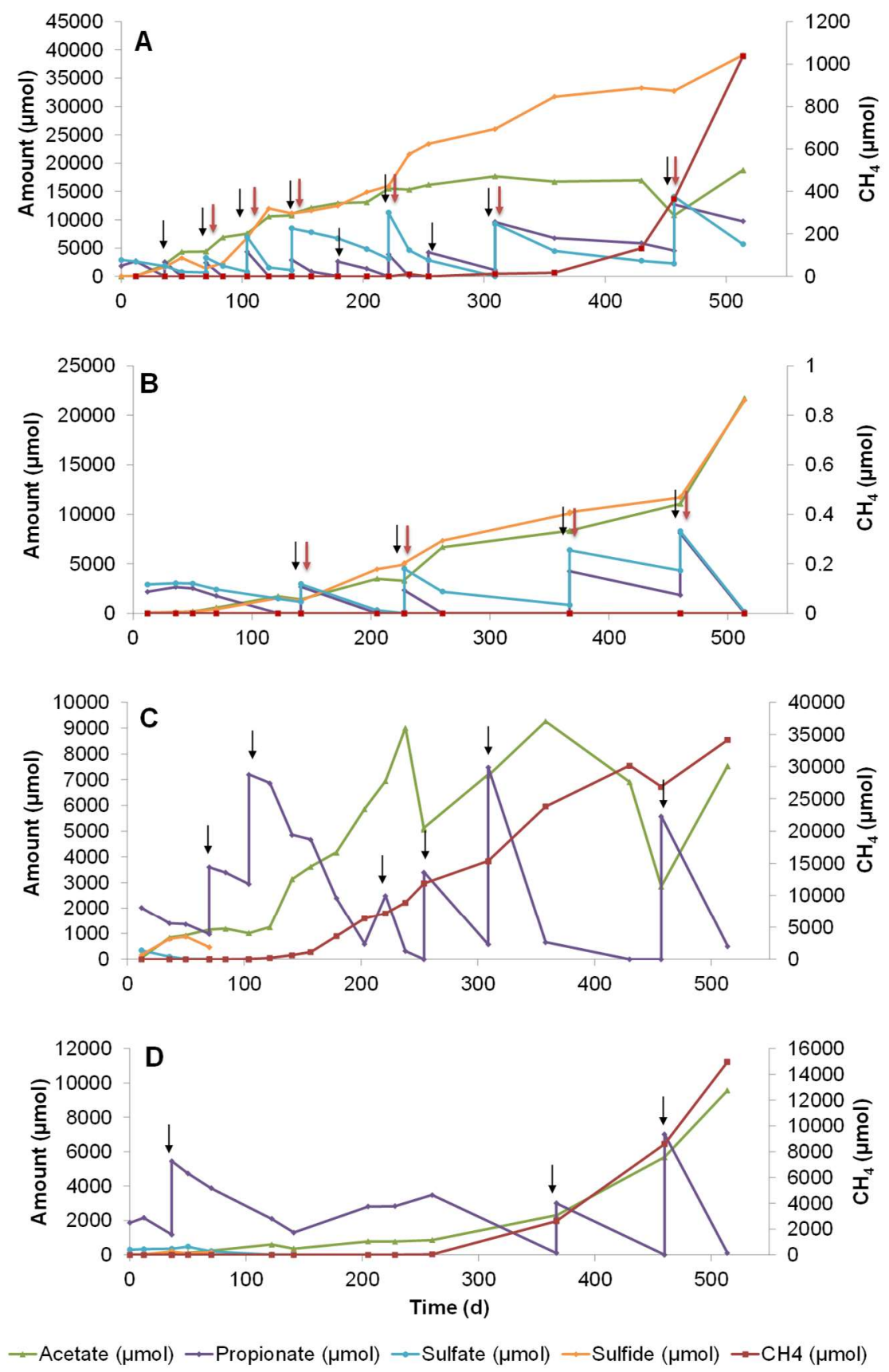

Figure 1. Changes in propionate, sulfate, acetate, sulfide and methane concentrations during 514 days of incubation in sediment slurry enrichments constituted of sulfate zone sediment (A) Slurry $\mathrm{P}$, with $20 \mathrm{mM}$ sulfate addition at $25^{\circ} \mathrm{C}$, (B) Slurry P8, with $20 \mathrm{mM}$ sulfate addition at $10^{\circ} \mathrm{C},(\mathrm{C})$ Slurry $\mathrm{P} 1$, without sulfate addition at $25^{\circ} \mathrm{C}$, (D) Slurry P6, without sulfate addition at $10^{\circ} \mathrm{C}$. Arrows denote the time points for the additions of sulfate (red) and propionate (black). 


\subsubsection{Sulfate-methane transition zone sediment slurries}

SMTZ sediments were treated with low $(3 \mathrm{mM})$ and high $(20 \mathrm{mM})$ concentrations of sulfate for 571 days at $25^{\circ} \mathrm{C}$ and $10^{\circ} \mathrm{C}$. Several re-feeds of propionate and sulfate were performed over the course of the experiment (Fig 2).
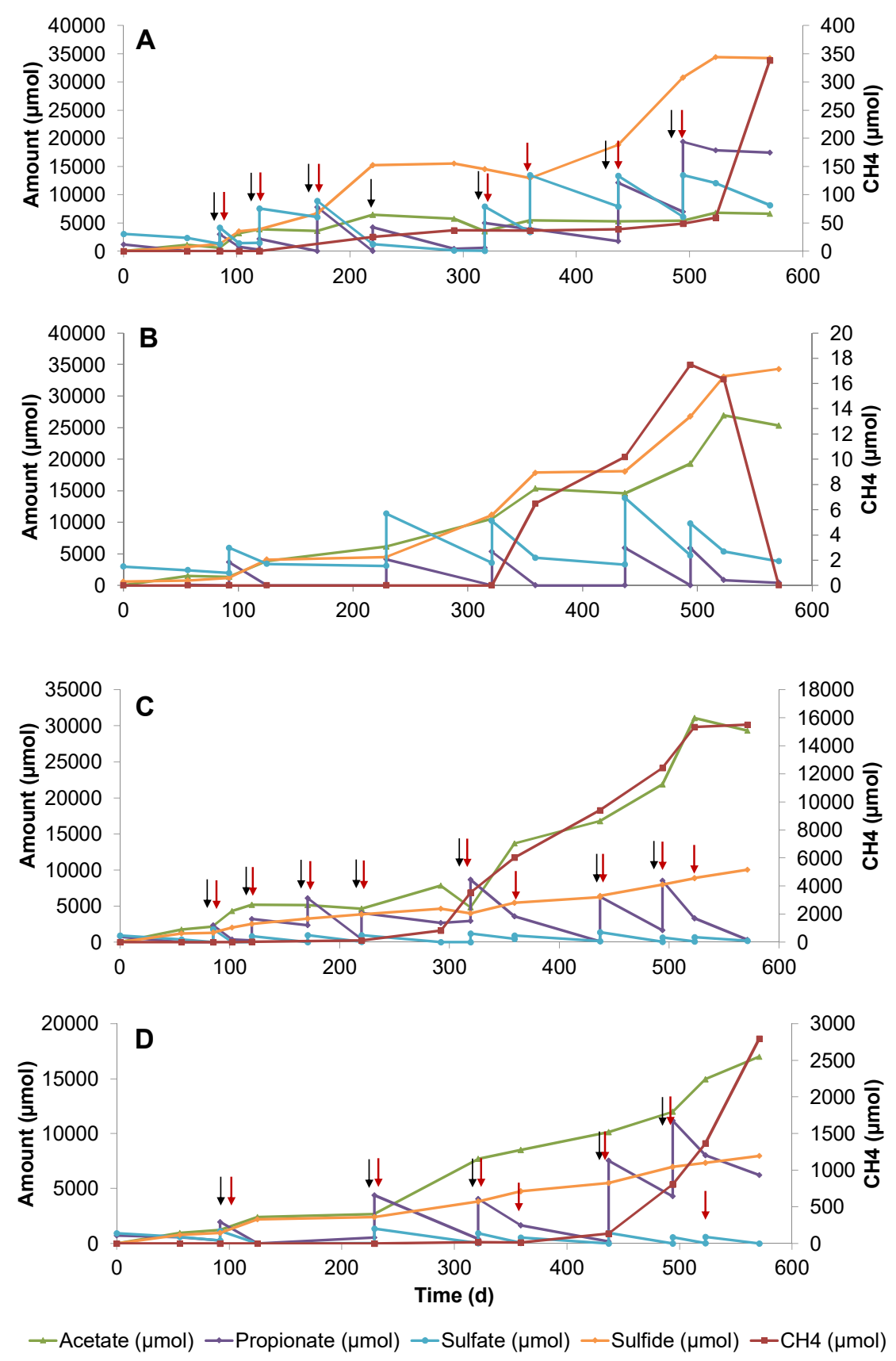

Figure 2. Changes in propionate, sulfate, acetate, sulfide and methane concentrations during 571 days of incubation in sediment slurry enrichments constituted of sulfate-methane transition zone sediment (A) Slurry $\mathrm{P}_{3}$, with $20 \mathrm{mM}$ sulfate addition at $25^{\circ} \mathrm{C}$, (B) Slurry $\mathrm{P}_{7}$, with $20 \mathrm{mM}$ sulfate 
addition at $10^{\circ} \mathrm{C}$ (C) Slurry $\mathrm{P}_{2}$, with $3 \mathrm{mM}$ sulfate addition at $25^{\circ} \mathrm{C}$, (D) Slurry $\mathrm{P}_{5}$, with $3 \mathrm{mM}$ sulfate addition at $10^{\circ} \mathrm{C}$. Arrows denote the time points for the additions of sulfate (red) and propionate (black).

In high sulfate treatments, the amount of consumed propionate was similar at both temperatures (Fig 2A, 2B). Propionate conversion coupled to sulfate reduction occurred and consequently the sulfide concentration increased. Acetate accumulated slowly in $25^{\circ} \mathrm{C}$ slurries (Fig 2A) whereas a steady increase in acetate was observed in $10^{\circ} \mathrm{C}$ slurries (Fig $2 \mathrm{~B}$ ). Methane in $25^{\circ} \mathrm{C}$ slurries was first observed on day 220, slightly increased until day 523, and a sudden rise was measured between days 523 and 571. On the other hand, methane formation in $10^{\circ} \mathrm{C}$ slurries was detected on day 359 and reached to $17 \mu \mathrm{mol} /$ bottle by the end of the experiment which was 20 times less than the methane that was produced in $25^{\circ} \mathrm{C}$ slurries.

The concentrations of propionate consumed and acetate accumulated in low sulfate amended slurries were two times higher at $25^{\circ} \mathrm{C}$ (Fig $2 \mathrm{C}$ ) as compared to the slurries incubated at $10^{\circ} \mathrm{C}$ (Fig 2D). Methane formation started on day 85 at $25^{\circ} \mathrm{C}$, gradually increased and reached to a concentration which was more than five times higher than that was measured in $10^{\circ} \mathrm{C}$ slurries.

\subsubsection{Methane zone sediment slurries}

The fastest propionate conversion in the methane zone slurries occurred under sulfate-amended conditions at $25^{\circ} \mathrm{C}$ (Fig 3A). Propionate conversion via sulfate reduction started in the beginning of the experiment and continued during the course of the study. Methane formation was observed on day 294 and boosted after day 414. However, the conversion of propionate at $10^{\circ} \mathrm{C}$ was slow and no methane production occurred during the experiment (Fig $3 \mathrm{~B}$ ).

The highest methane concentration was observed in the sulfate-free slurry at $25^{\circ} \mathrm{C}(\mathrm{Fig} 3 \mathrm{C})$. Approximately $1.1 \mathrm{mM}$ sulfate was measured in the slurry at the beginning of the experiment, and propionate conversion coupled to sulfate reduction occurred within the first 50 days. The depletion of sulfate in the slurry was followed by a lag phase where almost no change was observed in propionate and acetate levels. After day 107, propionate conversion started rapidly with concomitant acetate and methane production. Acetate accumulated until day 259 and then was almost completely consumed. Propionate conversion in methanogenic slurries at $10^{\circ} \mathrm{C}$ occurred via sulfate reduction until the trace amount of sulfate had depleted (Fig $3 \mathrm{D}$ ). Thereafter a lag phase of 226 days was observed which was followed by rapid propionate conversion accompanied by steep 
increase in acetate and methane. In total, 9 times less methane was produced at $10^{\circ} \mathrm{C}$ as compared to $25^{\circ} \mathrm{C}$ in sulfate-free slurries.
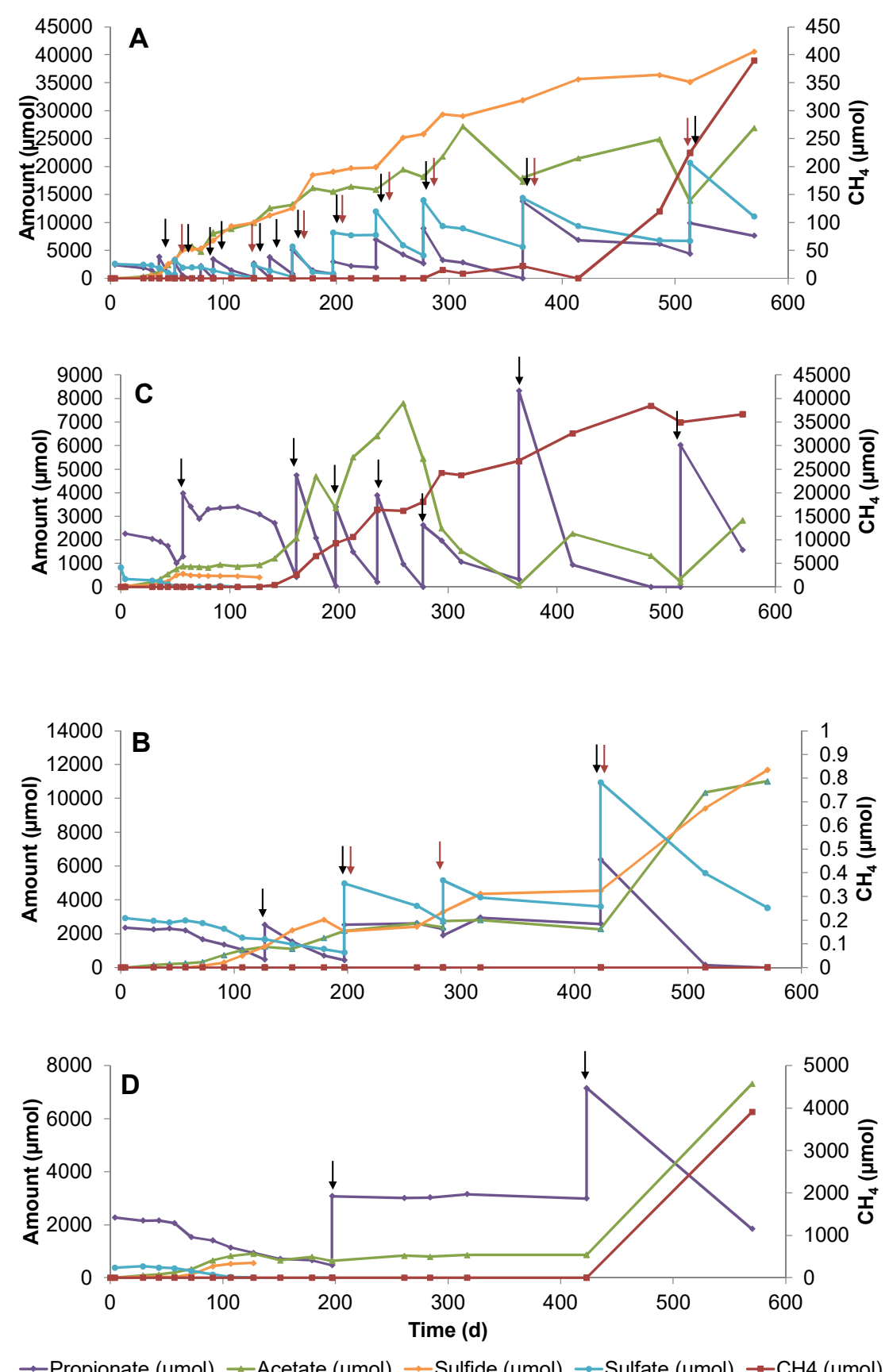

Figure 3. Changes in propionate, sulfate, acetate, sulfide and methane concentrations during 570 days of incubation in sediment slurry enrichments constituted of methane zone sediment (A) Slurry P6, with $20 \mathrm{mM}$ sulfate addition at $25^{\circ} \mathrm{C}$, (B) Slurry P8, with $20 \mathrm{mM}$ sulfate addition at $10^{\circ} \mathrm{C}(\mathrm{C})$ Slurry $\mathrm{P}_{3}$, without sulfate addition at $25^{\circ} \mathrm{C}$, (D) Slurry $\mathrm{P} 1$, without sulfate addition at $10^{\circ} \mathrm{C}$. Arrows denote the time points for the additions of sulfate (red) and propionate (black). 


\subsubsection{Distribution of bacterial and archaeal community through PCR-DGGE analysis}

Changes in the community structure over time in all slurries were visualized by denaturing gradient gel electrophoresis (DGGE). Bacterial DGGE gels harboured more bands in general compared to the archaeal gels. Some bands were present at all times with the same intensity, some of them disappeared or became fainter and some appeared and get intensified in time; revealing a dynamic bacterial community. In archaeal DGGE gels, most of the bands kept their position throughout the incubation period. Some bands became fainted while new bands appeared in methanogenic slurries especially after 100 days of incubation and thickened (data not shown). These banding patterns were organized in dendrograms by UPGMA clustering analysis (Fig S2, S3).

Clustering analysis of bacterial DGGE banding patterns gave separate clusters for each depth zone (i.e. SZ, SMTZ, MZ) except for the sample SZ P1 and SZ P8 which differed from all other samples (Fig S2). Methane zone slurries formed 4 sub-clusters; each slurry under different incubation condition formed a separate sub-cluster. Sulfate zone slurries formed 2 sub-clusters for each slurry, one representing the earlier incubation time samples and the other representing the later incubation time samples. SMTZ slurries formed two main sub-clusters, one representing the $25^{\circ} \mathrm{C}$ and the other $10^{\circ} \mathrm{C}$ slurries.

For the Archaeal communities, slurries belonging to each geochemical zone were grouped as one cluster similar to what was observed for Bacteria, except for the sub-clusters of three slurries belonging to the methane zone, MZ P3, P6, P8 (Fig S3). SMTZ slurries grouped based on incubation temperature, and one sub-cluster representing the samples of the last sampling time of each slurry differed from all other SMTZ samples. On the other hand, SZ slurries clustered depending on the sampling time. The slurries formed different sub-clusters as the community in the slurries differed by the enrichment of some metabolic groups.

\subsubsection{Bacterial community composition after long-term incubation}

PCR amplified partial 16S rRNA gene fragments obtained from the last sampling time of all slurries were sequenced. After filtering and trimming, between 1888 and 27196 high quality reads were found per sample (Table S2) and clustered into 64-131 operational taxonomic units (OTUs) per sample. 
OTUs classified into 33 phyla, with 95\% of the OTUs belonging to Proteobacteria (61.3\%), Firmicutes (17.5\%), Chloroflexi (9.8\%), Bacteroidetes (3.4\%), Spirochaetes (2.5\%) and Candidate division $\mathrm{OP}_{9}$ (1\%). The Desulfobacteraceae and Desulfobulbaceae were the two dominant families in the phylum Proteobacteria, having $38.6 \%$ and $29.2 \%$ of the reads, respectively (Fig S4).The Firmicutes phylum was dominated by the Peptococcaceae family comprising $86.8 \%$ of the reads. Similarly, the Anaerolinaceae family dominated the Chloroflexi phylum containing $78.1 \%$ of the reads (Fig $\mathrm{S}_{4}$ ). Above mentioned four families were the most dominant in all samples. Despite the overall dominance of these families, a high degree of variation was observed in their relative abundances between different slurries.

The most abundant OTUs in different slurries belonged to the genera Cryptanaerobacter (76\%) in the phylum Firmicutes, Desulfobulbus (59\%), Desulfosarcina (52\%), SEEP-SRB1 (45\%) and Desulforhopalus (44\%) in the phylum Proteobacteria (Fig 4). Additionally, the most abundant OTUs (85\%) in one slurry (SZ P6) belonged to the family Desulfobacteraceae which could not be assigned to any genus (Fig 4). 


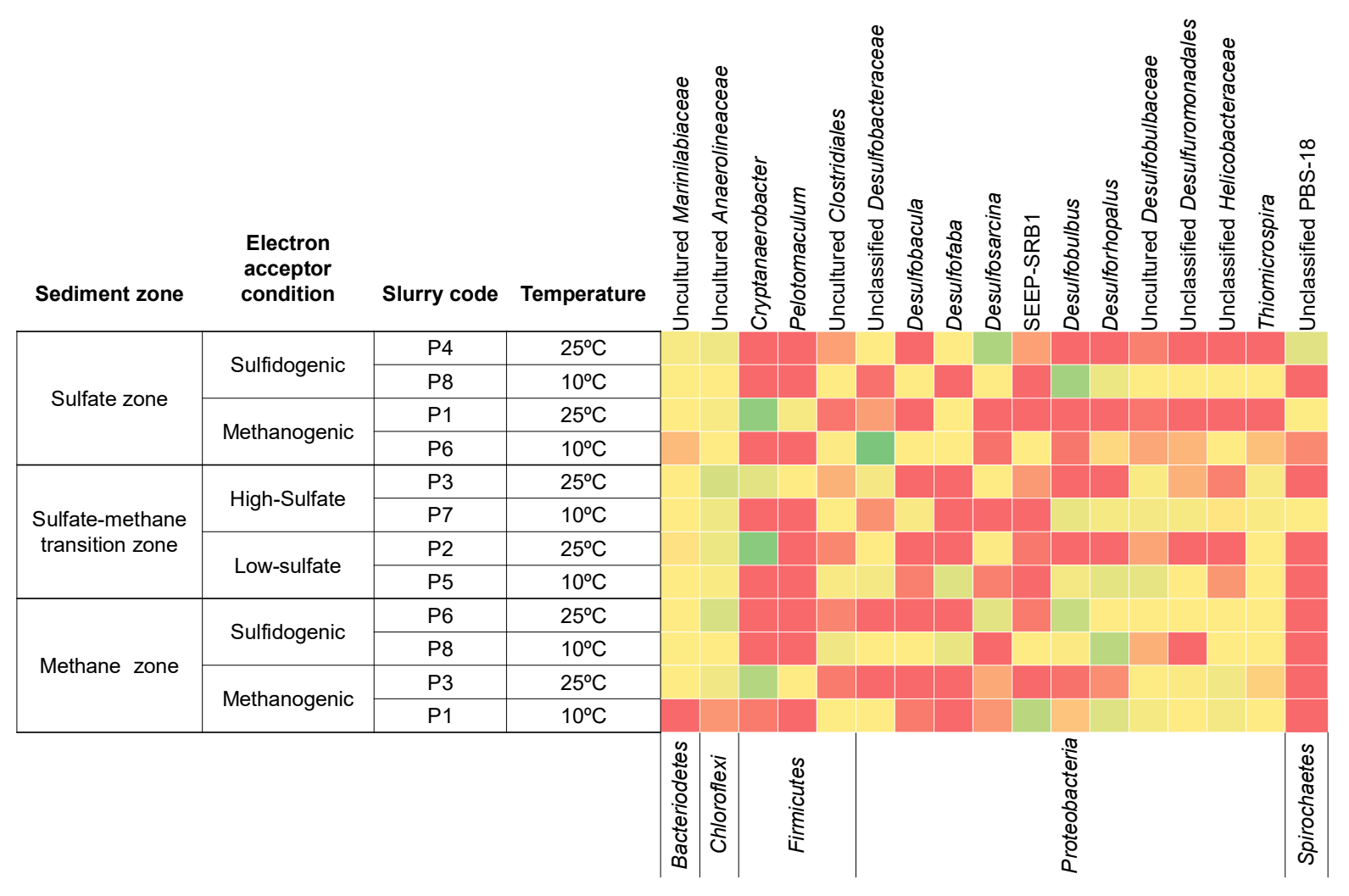

$\begin{array}{llllllllll}0 & 2.5 & 5 & 7.5 & 10 & 20 & 30 & 50 & 75 & 100\end{array}$

Figure 4. The heatmap depicts the relative percentage of the most common ( $>5 \%$ ) bacterial $16 \mathrm{~S}$ rRNA gene sequences across the 12 slurries fed with propionate.The heatmap colors represent the relative percentage of the bacterial assignments within each sample. Colors shifted towards dark green indicate higher abundance. Taxonomy is shown at the genus level (unless unclassified) above and at the phylum level below the heatmap.

The microbial community was analysed using redundancy analysis (RDA) (Fig 5). The bacterial community composition in RDA was plotted on the basis of the relative read abundance of OTUs in each sample. The highest read abundance, in decreasing order, belonged to the bacterial orders Desulfobacterales, Clostridiales and Anaerolineales with OTU numbers 26, 19 and 15, respectively (Fig 5). 


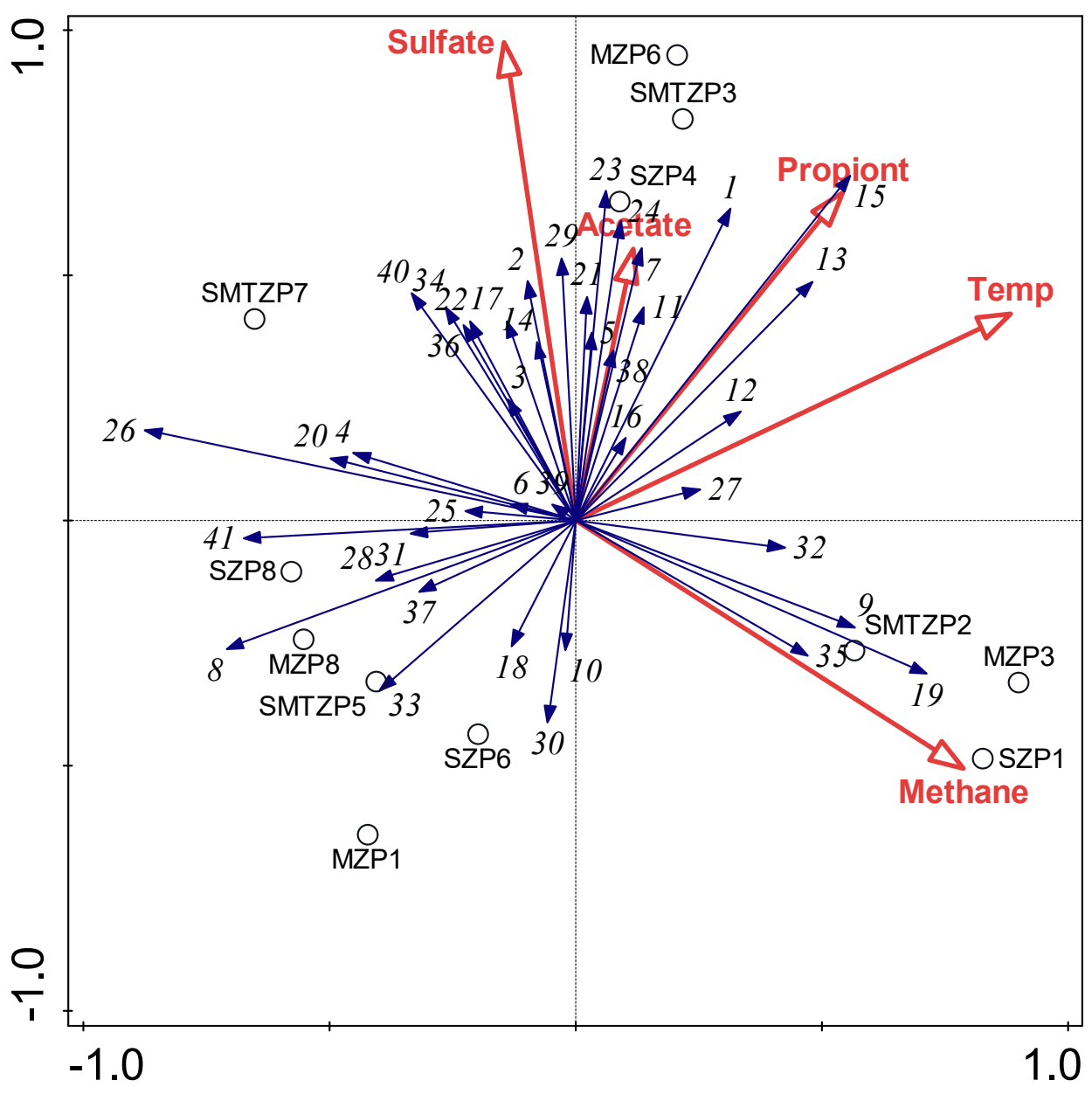

Figure 5. Redundancy analysis triplot showing relationship between bacterial community composition at order level and environmental parameters. Environmental variables are given as red vectors. Blue vectors represent bacterial orders. Orders were included with a relative abundance of at least $1 \%$ in any sample. Vector length gives the variance that can be explained by a particular environmental parameter. Perpendicular distance reflects association, with smaller distances indicating a larger association. Temp: Temperature, Propiont: Propionate.

OTU numbers and corresponding taxa are as followed: (1)Bacteria-Other; (2)Actinobacteria-OPB41; (3)Bacteroidetes; (4)Bacteroidetes-BD2-2; (5)Bacteroidetes-Bacteroidales; (6)BacteroidetesFlavobacteriales; (7)Bacteroidetes-SB-1; (8)Bacteroidetes-SB-5; $\quad$ (9)BacteroidetesSphingobacteriales; (10)Bacteroidetes-VC2.1.Bac22; (11)Bacteroidetes-vadinHA17; (12)BacteroidetesvadinHAr7-uncultured bacterium; (13)Candidate division OP9; (14)Chloroflexi-Anaerolineae; (15)Chloroflexi-Anaerolineales; (16)Chloroflexi-GIF9; (17)Cyanobacteria-Chloroplast; (18)Elusimicrobia-Lineage_IV; （19)Firmicutes-Clostridiales; （20)Nitrospirae-Nitrospirales; (21)Planctomycetes-Planctomycetales; (22)Proteobacteria-Rhizobiales; (23)ProteobacteriaBurkholderiales; (24)Proteobacteria-Deltaproteobacteria; (25)Proteobacteria-Desulfarculales; (26)Proteobacteria-Desulfobacterales; (27)Proteobacteria-Desulfovibrionales; (28)ProteobacteriaDesulfuromonadales; (29)Proteobacteria-Svao485; （30)Proteobacteria-Campylobacterales; (31)Proteobacteria-Gammaproteobacteria; (32)Proteobacteria-Alteromonadales; (33)Proteobacteria-Chromatiales; (34)Proteobacteria-Thiotrichales; (35)RF3; (36)RF3-uncultured 
bacterium; (37)Spirochaetes-LK-44f; (38)Spirochaetes-PBS-18; (39)Spirochaetes-Spirochaetaceae; (40)TM6; (41)Tenericutes-Acholeplasmatales.

The OTUs affiliating with the order Desulfobacterales were associated with the slurries incubated at $10^{\circ} \mathrm{C}$ in which they were dominant. These slurries showed close clustering towards the bottom left part of the RDA triplot and they were negatively correlated with the temperature. The second most abundant order, Clostridiales, showed strong association with methane and was plotted between the slurries $\mathrm{SZP}_{1}, \mathrm{SMTZP}_{2}$ and $\mathrm{MZP}_{3}$ in which the highest methane concentration was measured. These three slurries were incubated at $25^{\circ} \mathrm{C}$ and without or low sulfate which was supported by a negative association to sulfate and a positive association to temperature. Another abundant order, Anaerolineales, associated with high sulfate and high temperature conditions clustering in the upper right portion of the graph between temperature and sulfate, adjacent to the slurries $\mathrm{SZP}_{4}, \mathrm{MZP6}, \mathrm{SMTZP}_{3}$ incubated under these conditions (Fig 5, $\mathrm{S}_{4}$ ).

\subsubsection{Archaeal community composition and structure}

PCR amplified partial 16S rRNA gene fragments obtained from the slurries on the last sampling day were sequenced. After filtering and trimming, between 5547 and 108810 high quality reads were found per sample.

The highest percentage of $16 \mathrm{~S}$ rRNA reads for Archaea clustered within the families Methanomicrobiaceae (39.8\%), an unclassified Methanomicrobiales clone (EJ-Eo1) (21.4\%) belonging to Methanomicrobiales, Marine Benthic Group D (MBG-D) and DHVEG-1 (10.7\%), Methanosarcinaceae (10.4\%) and unclassified Methanomicrobiales (9.7\%) (Fig S6). Methanoculleus and Methanogenium from the family Methanomicrobiaceae dominated different slurries (Fig 6). The unclassified Methanomicrobiales clone (EJ-Eo1) dominated three slurries where the relative abundances of Methanoculleus and Methanogenium were low. Methanosarcina was observed to be the dominant genus being present in four slurries with read percentages ranging between 14-30\%, whereas Methanoccoides had less reads in seven slurries. Marine Benthic Group D (MBG-D) and DHVEG-1 from the order Thermoplasmatales dominated two methane zone slurries containing sulfate with read percentages $45 \%$ and $47 \%$ (Fig 6 ). 


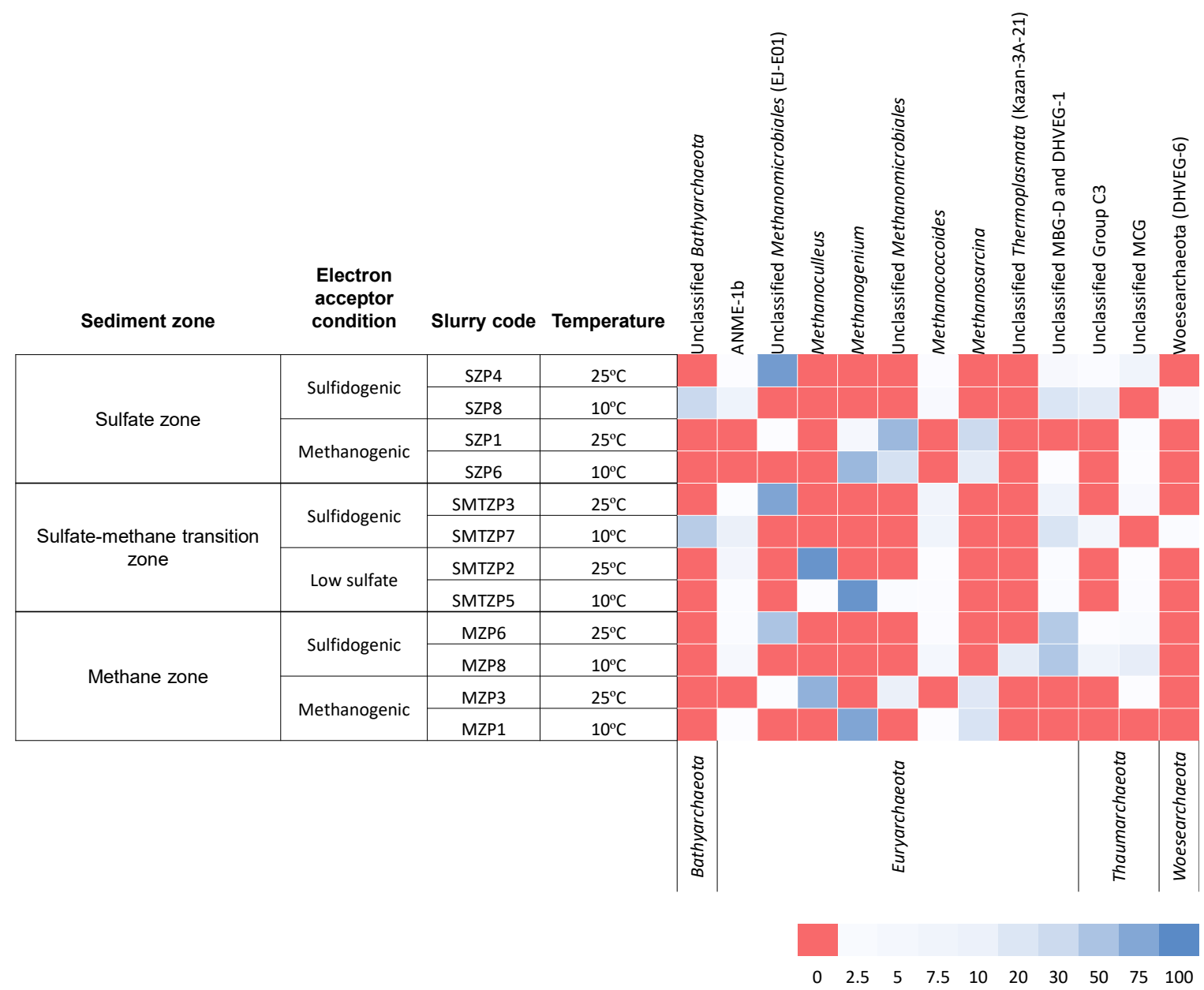

Figure 6. The heatmap depicts the relative abundance of the most common ( $>5 \%$ ) archaeal $16 \mathrm{~S}$ rRNA gene sequences (unless unclassified) across the 12 slurries fed with propionate.The heatmap colors represent the relative percentage of the archaeal assignments within each sample. Colors shifted towards bright blue indicate higher abundance.

Archaeal RDA triplots showed that the slurries originating from the same biogeochemical zone were not clustered closely which indicated that the community composition changed upon incubation (Fig 7). A strong association was observed between methane and the families Methanomicrobiaceae, unclassified Methanomicrobiales and Methanosarcinaceae, and negative association between sulfate and these taxa. The slurries SZP6, MZP1, SMTZP2 and SMTZP5, which were dominated with Methanomicrobiaceae, showed close clustering (Fig 7, S6). The other two slurries, $\mathrm{SZP}_{1}$ and $\mathrm{MZP}_{3}$, were strongly associated with methane and showed close association with other two methanogenic families Methanosarcinaceae and unclassified Methanomicrobiales. The unclassified Methanomicrobiales clone (EJ-Eo1) was prevalent in the slurries containing sulfate and was plotted between the sulfidogenic slurries $\mathrm{SMTZP}_{3}, \mathrm{SZP}_{4}$ and MZP6 (Fig 7, Fig S6). MBG-D and 
DHVEG-1 showed a close relation to sulfate and was plotted close to the sulfidogenic slurries MZP6 and MZP8 in which they were dominantly present.

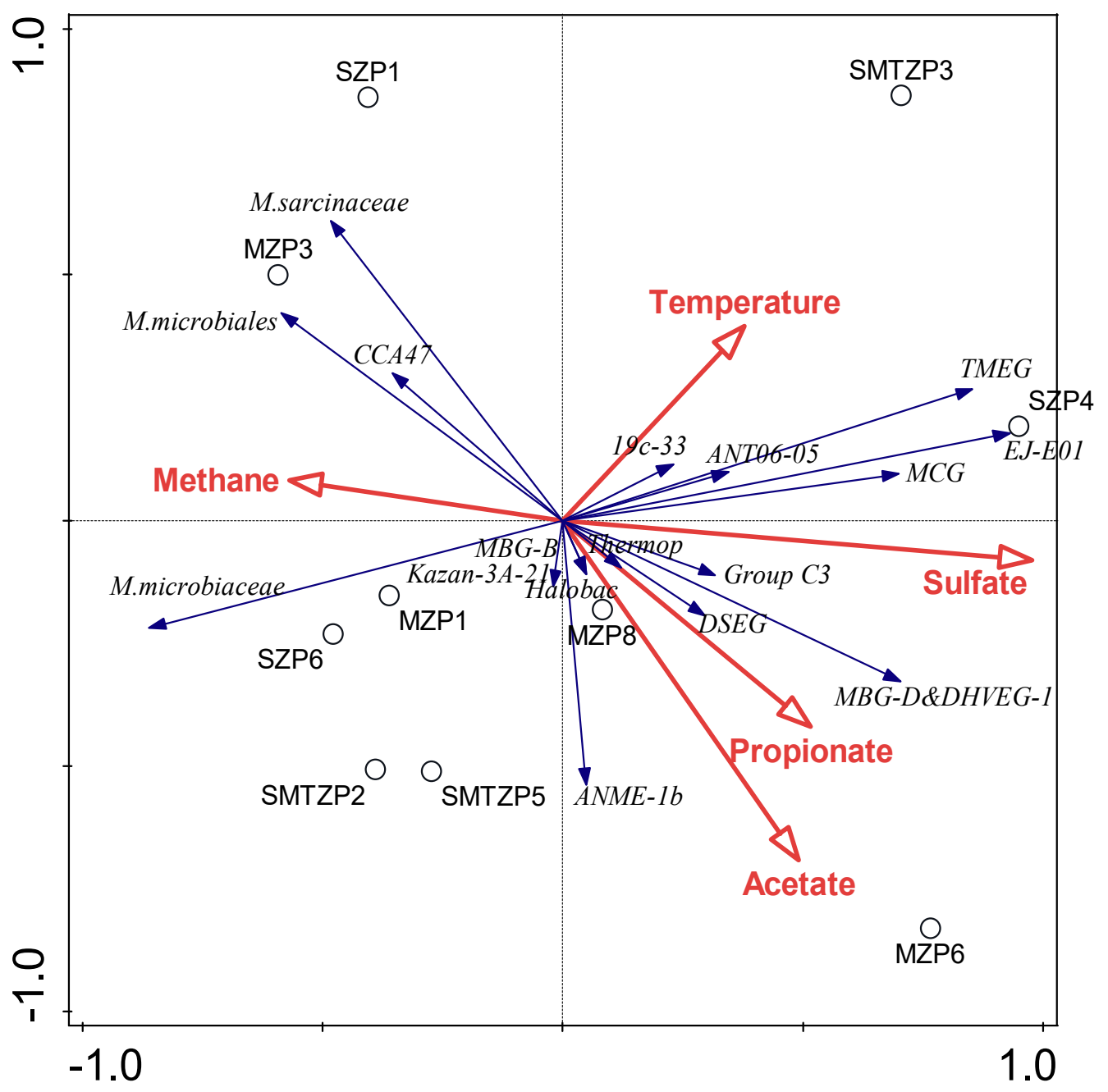

Figure 7. Redundancy Analysis Triplot showing relationship between Archaeal community composition at family level and environmental parameters. Environmental variables are given as red vectors. Blue vectors represent Archaeal families. Families were included with a relative abundance of at least $1 \%$ in any sample. Vector length gives the variance that can be explained by a particular environmental parameter. Perpendicular distance reflects association, with smaller distances indicating a larger association. Full names of the phylotypes in the plot are as followed: M.sarcinanaceae: Methanosarcinaceae; M.microbiales: Methanomicrobiales; M.microbiaceae: Methanomicrobiaceae; Halobac: Halobacteriales; Thermop: Thermoplasmatales. 


\subsubsection{Correlation within the microbial community and between the microbial community and environmental parameters}

Correlation analysis of the sequencing data revealed both positive and negative relations between 34 bacterial orders that were present in different slurries (Fig $\left.\mathrm{S}_{5}\right)$. Clostridiales had a strong negative correlation to Desulfobacterales order $(P<0.05)$ and to sulfate and sulfide, however showed a positive correlation to temperature and methane. An overall negative correlation was observed between Desulfobacterales and the orders Clostridiales $(P<0.05)$, Anaerolineales $(P<0.05)$, candidate division $\mathrm{OP}_{9}(P<0.05)$, and the incubation temperature $(P<0.01)$. Strong positive correlations were observed between Anaerolineales and temperature $(P<0.01)$, VFA $(P<0.01)$, propionate $(P<$ o.o1 $)$. Unclassified Deltaproteobacteria showed positive correlation to the orders SB-1 $(P<$ o.o1), Flavobacteriales, Anaerolineales, candidate division OP9 and to all environmental parameters except for methane (Fig $\mathrm{S}_{5}$ ).

The hydrogenotrophic methanogenic family Methanomicrobiaceae and unclassified Methanomicrobiales order showed similar trend in their correlation to other taxa and environmental parameters except for few differences (Fig S7). Methanomicrobiaceae family was the only family showing positive correlation to the ANME-1b and strong positive correlation to methane production $(P<0.05)$. On the other hand, unclassified Methanomicrobiales was positively correlated to Methanosarcinaceae. Differently from other methanogenic groups, the unclassified Methanomicrobiales (EJ-Eor clone) showed positive correlations to some families belonging to Thermoplasmata and Thaumarchaeota phyla and all environmental parameters except for methane. MBG-D and DHVEG-1 was positively correlated to most of the taxonomic groups and environmental parameters, whereas strong negative correlations were observed to methane and the methanogenic taxa $(P<0.05)$, except for the unclassified Methanomicrobiales (Fig $\left.\mathrm{S}_{7}\right)$.

\subsection{Discussion}

\subsubsection{Sulfate-dependent propionate conversion}

Propionate is an important substrate for sulfate reducers in marine sediments, providing about 10\% of the reducing equivalents for sulfate reduction (Sørensen et al., 1981). The propionate conversions in sulfate amended SZ and MZ slurries at $25^{\circ} \mathrm{C}$ were similar in terms of immediate start and conversion rate of propionate (Fig $1 \mathrm{~A}, 3 \mathrm{~A})$. The stoichiometry of the propionate conversion in $\mathrm{MZ}$ slurries was determined as $\mathrm{CH}_{3} \mathrm{CH}_{2} \mathrm{COO}^{-}+0.9 \mathrm{SO}_{4}{ }^{2-} \rightarrow \mathrm{CH}_{3} \mathrm{COO}^{-}+0.8 \mathrm{HS}^{-}$, and this pointed to 
incomplete propionate oxidation (Table 1, reaction 2). On the other hand, the ratio of reduced sulfate to the consumed propionate in SZ slurry $\left(\mathrm{CH}_{3} \mathrm{CH}_{2} \mathrm{COO}^{-}+1.6 \mathrm{SO}_{4}{ }^{2-} \rightarrow \mathrm{CH}_{3} \mathrm{COO}^{-}+1.5 \mathrm{HS}^{-}\right)$is close to the theoretical values of complete propionate conversion (Table 1, reactions 2+4) (Table $\mathrm{S} 1$ ). Especially between the days 220 and 430, propionate was completely converted coupled to sulfate reduction and no net acetate increase was observed (Fig $1 \mathrm{~A}$ ). The high concentration of acetate detected at the end of the incubation period might have originated from the metabolism of fermentative bacteria. Yet, simultaneous increase in acetate and methane between the days 457 and 514 suggests syntrophic conversion of propionate by fermentative bacteria and hydrogenotrophic methanogenic archaea, in addition to the ongoing sulfate-dependent propionate conversion (Fig 1A). It was striking to detect rapidly increasing methane in MZ as well as SZ slurry after 414 and 309 days of incubation, respectively $\left(\mathrm{Fig}_{1} \mathrm{~A}, 3 \mathrm{~A}\right)$. The late methane production might be linked to the sulfide inhibition of methanogens which was reported in previous studies (Pender et al., 2004; O'Flaherty et al., 1998; Shin et al., 1995). Despite high sulfidogenic activity, methanogenic archaea might have adapted to the slurry conditions and be involved in the consumption of conversion products.

The ratio of consumed propionate and sulfate in high-sulfate amended SMTZ slurry at $25^{\circ} \mathrm{C}$ $\left(\mathrm{CH}_{3} \mathrm{CH}_{2} \mathrm{COO}^{-}+1.3 \mathrm{SO}_{4}{ }^{2-} \rightarrow 0.3 \mathrm{CH}_{3} \mathrm{COO}^{-}+1.2 \mathrm{HS}^{-}\right)$points to the combination of incomplete and complete propionate oxidation via sulfate reduction. The acetate that was formed as primary product from incomplete conversion was further oxidized by aceticlastic SRB (Table S1, Fig 2A). Propionate in the low sulfate-amended slurry at $25^{\circ} \mathrm{C}$ was converted by both sulfate reducers and syntrophs in cooperation with methanogens $\left(\mathrm{CH}_{3} \mathrm{CH}_{2} \mathrm{COO}^{-}+0.3 \mathrm{SO}_{4}{ }^{2-} \rightarrow 1.1 \mathrm{CH}_{3} \mathrm{COO}^{-}+0.3 \mathrm{HS}^{-}+\right.$ $0.5 \mathrm{CH}_{4}$ ) (Table S1, Fig $2 \mathrm{C}$ ). The low levels of sulfate were not sufficient to support complete propionate conversion through sulfate reduction and consequently syntrophic propionatedegrading communities became active (Muyzer and Stams, 2008).

\subsubsection{Sulfate-independent propionate conversion}

Propionate conversion in sulfate-free SZ and MZ slurries at $25^{\circ} \mathrm{C}$ started with the reduction of sulfate originating from the sediment ( $F i g{ }_{1} C,{ }_{3} C$ ). This conversion indicated the existence of metabolically active sulfate-reducing microorganisms in sulfate zone as well as in methane zone. Previous studies reported the presence of active SRB with similar cell numbers in the upper and lower parts of the coastal marine sediments of Limfjorden and Aarhus Bay, Denmark (Jørgensen, 1978; Thomsen et al., 2001; Leloup et al., 2009) and deep-sea sediment (Leloup et al., 2007). The 
high abundance of SRB in sulfate-depleted sediments might be due to the acetogenic and fermentative growth characteristics of some SRB in the absence of sulfate (Plugge et al., 2011; Muyzer and Stams, 2008). The fast propionate conversion with concomitant acetate and methane production in both SZ and MZ slurries suggests the activity of syntrophic propionate-converting consortia (Fig $1 \mathrm{C},{ }_{3} \mathrm{C}$ ). Under methanogenic conditions, the degradation of propionate to acetate, $\mathrm{CO}_{2}$, and ${ }_{3} \mathrm{H}_{2}$ is highly endergonic process $\left(\Delta \mathrm{G}^{\circ}=76.1 \mathrm{~kJ} / \mathrm{mol}\right)$ (Table 1 ), but it can be accomplished by syntrophic cooperation of propionate-oxidizing bacteria and $\mathrm{H}_{2^{-}}$or formate-consuming methanogens (Boone and Bryant, 1980; McInerney et al., 2008). Acetate rapidly accumulated in the slurries as a result of propionate conversion, and was utilized only after propionate became depleted (Fig $1 \mathrm{C},{ }_{3} \mathrm{C}$ ). Similar conversion dynamics were reported for both methanogenic (Viggi et al., 2014; Stams et al., 1992) and sulfidogenic batch cultures (Laanbroek and Pfennig, 1981). Apparently, acetoclastic methanogens contributed to the conversion process by consuming accumulated acetate after propionate amount became low in both slurries (Fig ${ }_{1} \mathrm{C},{ }_{3} \mathrm{C}$ ). Archaeal DGGE profiles of SZ slurry reflected appearance of new bands on day 138 and 358 (data not shown) after methane production and acetate consumption started, respectively (Fig $1 \mathrm{C}$ ). Clustering analysis placed these two time samples in different sub-clusters indicating the change in archaeal community structure along the incubation (Fig S3). In MZ slurry, acetate was consumed down to near zero value, showing efficient cooperation of syntrophic bacteria, hydrogenotrophic and acetoclastic methanogenic archaea.

\subsubsection{The effect of temperature on propionate conversion}

Low incubation temperature have an impact on propionate conversion both in the presence and absence of sulfate in Aarhus Bay sediments. Propionate conversion at $10^{\circ} \mathrm{C}$ occurred overall slower and methane was not detected in sulfate amended SZ and MZ slurries (Fig $1 \mathrm{~B},{ }_{3} \mathrm{~B}$ ). More acetate accumulated in high-sulfate SMTZ slurry at $10^{\circ} \mathrm{C}$ as compared to its replicate at $25^{\circ} \mathrm{C}$. This might be linked to the low activity of acetoclastic microorganisms. Previous experiments performed with marine sediments in sulfate-amended slurries showed that the rate of sulfate reduction decreased and as a result, organic acid concentration increased at temperatures below $25^{\circ} \mathrm{C}$ (Weston and Joye, 2005).

In sulfate-free $\mathrm{MZ}$ slurry at $10^{\circ} \mathrm{C}$, a long lag phase was observed between the consumption of trace amount of sulfate and the start of propionate conversion (Fig ${ }_{3} \mathrm{D}$ ). It is known that microorganisms in subsurface environments have low metabolic and growth rate, and can persist 
in a dormant state (Jørgensen and Marshall, 2016). On the other hand, the distance between hydrogen-producing and hydrogen-consuming microorganisms during syntrophic degradation of a compound is important (Stams, 1994). Grotenhuis et al. (1991) observed $\mu \mathrm{m}$ range between propionate-oxidizing bacteria and methanogens in propionate-adapted methanogenic granules. Therefore, the slow growth and the establishment of the microbial clusters might have led to a late start of the syntrophic conversion of propionate at low temperature.

\subsubsection{Bacteria enriched in sulfate-amended slurries}

The most abundant sulfate-reducing genera in sulfate-amended SZ and MZ slurries at $25^{\circ} \mathrm{C}$ were Desulfosarcina belonging to Desulfobacteraceae and Desulfobulbus belonging to Desulfobulbaceae (Fig 4, 4S). The Desulfobacteraceae family mainly consist of sulfate reducers that completely oxidize organic substrates (Kuever, 2014b) and is commonly found as the dominant SRB in anoxic marine sediments (Dhillon et al. 2003; Llobet-Brossa et al. 2002; Leloup et al. 2007 and 2009; Jørgensen and Bak, 1991). Leloup et al. (2009) reported that the upper sulfate-rich sediment of Station M1, Aarhus Bay was dominated by Desulfosarcina species. Similar findings on the predominance of Desulfosarcina-like dsrAB sequences were observed at Station 6 in Aarhus Bay (Sahm et al., 1999), in Kysing Fjord, Denmark, (Thomsen et al., 2001) and Mariager Fjord, Denmark (Wagner et al., 2005). Desulfosarcina species are able to use propionate as electron donor (Widdel, 1980). Thus they participated in propionate conversion in sulfate-amended sediment slurry enrichments at $25^{\circ} \mathrm{C}$.

Sulfate-amended slurries at $10^{\circ} \mathrm{C}$ were dominated by Desulfobulbus and Desulforhopalus (Fig 4, 4S). The relative abundance of Desulfobulbus decreased, whereas Desulforhopalus increased with increasing depth. Low temperature SMTZ slurries also contained Desulfofaba, belonging to Desulfobacteraceae. Desulfofaba species are known as obligately psychrophilic marine sulfatereducers capable of propionate oxidation (Kuever, 2014b). Most members of Desulfobulbaceae are incomplete oxidizers and specialized in the oxidation of organic acids, including propionate, to acetate (Kuever, 2014C; Muyzer and Stams; 2008; Devereux et al., 1989; Widdel and Bak, 1992). This is in agreement with the observed acetate accumulation in the Desulfobulbaceae containing slurries. The higher relative abundance of Desulfobulbaceae and some Desulfobacteraceae members in low temperature slurries is in line with the statistical analysis showing the negative correlation between the order Desulfobacterales and the incubation temperature (Fig $\mathrm{S}_{5}$ ). Apparently, the 
Desulfobulbaceae members had the competitive advantage and dominated over Desulfobacteraceae in low temperature slurries.

All sulfidogenic slurries incubated at $25^{\circ} \mathrm{C}$ contained uncultured Anaerolineaceae belonging to the phylum Chloroflexi (Fig 4, $\mathrm{S}_{4}$ ). The cultured representatives of this lineage are known as filamentous, slow-growing and strictly anaerobic chemoorganotrophs decomposing carbohydrates and amino acids (Yamada and Sekiguchi, 2009). It was reported that Anaerolineaceae members act as secondary degraders and degrade dead biomass together with acetate and $\mathrm{H}_{2}$-consuming methanogens (Kleinsteuber et al., 2012). Therefore, the uncultured Anaerolineaceae in our study might have formed a syntrophic relationship with hydrogenotrophic and/or acetoclastic methanogens and took part in the degradation of organic content and/or dead biomass.

\subsubsection{Bacteria enriched in sulfate-free slurries}

Sulfate-free and low-sulfate amended slurries composed of sediment from each biogeochemical zone and incubated at $25^{\circ} \mathrm{C}$ are dominated by Cryptanaerobacter and also contain Pelotomaculum both of which belong to the family Peptococcaceae (Fig 4, Fig S4). Currently known propionateoxidizing syntrophic species of the genus Pelotomaculum are P. schinkii, $P$. thermopropionicum, and P. propionicicum (de Bok et al., 2005; Imachi et al., 2002, 2007). The Pelotomaculum enriched in our study apparently takes part in propionate conversion. On the other hand, there is only one cultured species of the genus Cryptanaerobacter, namely $C$. phenolicus, and it transforms phenol and 4hydroxybenzoate (4-OHB) into benzoate (Juteau et al., 2005). Its closest cultured relative is Pelotomaculum thermopropionicum. Propionate utilization in the presence of a hydrogenotrophic methanogen by this genus has not been reported. We hypothesize that, Cryptanaerobacter might have the capability to convert propionate in syntrophy with hydrogenotrophic methanogens. The idea behind this hypothesis is based on ; i) the isolation of $C$. phenolicus from a methanogenic consortium, ii) the syntrophic lifestyle of its closest cultured relatives and iii) the presence of propionate as a sole carbon source in the slurries. Additionally, the hypothesis is supported with the statistical analysis showing positive correlation of the order Clostridiales with methane and temperature (Fig $\left.\mathrm{S}_{5}\right)$. 


\subsubsection{Sulfate-reducing bacteria enriched in sulfate-free slurries}

Bacterial community analysis revealed the dominance of unclassified Desulfobacteraceae members constituting $85 \%$ of the total reads in low-temperature sulfate-free SZ slurry (Fig 4, S4). Although it was surprising to observe a sulfate-reducing family representing the substantial part of the reads in sulfate-free slurry, there are non-sulfate reducing members of this family, such as the butyrate-utilizing marine syntroph Algorimarina butyrica (Kendall et al., 2006). In addition, Ulrich and Edwards (2003) enriched benzene-degrading cultures which were dominated by a phylotype affiliated to Desulfobacteraceae, and which were capable of switching from sulfate-reducing to methanogenic life style. Raskin et al. (1996) also reported the presence of Desulfovibrio spp. and Desulfobacterium spp. in a methanogenic reactor and explained this occurrence by their ability to function as proton-reducing acetogens and/or fermenters. These results illustrate the significance of metabolic flexibility of microorganism under changing conditions such as temperature, electron acceptor availability, the presence/absence of partner organisms.

Similar to the above mentioned SZ slurry, sulfate-free MZ slurry incubated at $10^{\circ} \mathrm{C}$ contained high numbers of Desulfobacteraceae-related reads (Fig 4). These reads were assigned as SEEP-SRB1, defining Desulfosarcina/Desulfococcus branch of Desulfobacteraceae. SEEP-SRB1 clade has been shown to live together with ANME-2 and ANME-1 in a syntrophic consortium in AOM-mediating environments and enrichments (Harrison et al., 2009; Vigneron et al., 2013; Timmers et al., 2015). The members of this group have been reported to have low sequence similarity to the cultivated species which points to new species or genera with unknown physiological properties (Knittel et al., 2003). As this slurry contains ANME-1b related reads, SEEP-SRB1 might have formed consortia with this archaeal cluster and involved in anaerobic oxidation of methane (Fig 3D, Fig 6).

\subsubsection{Archaea involved in propionate conversion}

Archaeal community analyses revealed the dominance of the order Methanomicrobiales in almost all slurries. The members of Methanomicrobiales utilize $\mathrm{H}_{2}+\mathrm{CO}_{2}$ and most members also use formate as substrate for methanogenesis (Garcia et al., 2006). This suggests that $\mathrm{H}_{2}+\mathrm{CO}_{2}$ and/or formate was the main substrate used by methanogenic Archaea in the slurries (Fig 6, S6). Hydrogenotrophic methanogenesis has been detected in near-surface marine sediments (Parkes et al., 2007; Webster et al., 2009; Sørensen et al. 1981; Blair and Carter 1992). Different genera or groups of Methanomicrobiales increased in relative abundance in the slurries based on incubation 
temperature and sulfate availability. Methanoculleus was observed in the low sulfate-amended SMTZ and sulfate-free MZ slurries incubated at $25^{\circ} \mathrm{C}$, whereas Methanogenium comprised most of the sequences in SZ, SMTZ and MZ slurries incubated at $10^{\circ} \mathrm{C}$ without or with low sulfate amendment (Fig 6). The optimum growth temperature of Methanoculleus ranges between $25^{\circ} \mathrm{C}$ and $60^{\circ} \mathrm{C}$, whereas Methanogenium species grow best between $15-35^{\circ} \mathrm{C}$. Therefore, temperature might have been a determining factor for the dominance of different genera. On the other hand, high sulfate-amended slurries incubated at $25^{\circ} \mathrm{C}$ contained predominantly unclassified Methanomicrobiales sequences, termed as EJ-Eo1 (Fig 6, Fig S6). It is obvious that the increased relative abundance of this unclassified hydrogenotrophic methanogenic group was closely related to the presence of sulfate, as was approved by its positive correlation with sulfate and temperature (Fig S7). Hydrogenotrophic methanogenesis in high sulfate containing sediments has also previously been detected in marine sediments (Kendall and Boone, 2006a; Parkes et al., 1990).

Methanosarcina belonging to the Methanosarcinaceae that utilize acetate, methylated compounds and $\mathrm{H}_{2}+\mathrm{CO}_{2}$ detected (Kendall and Boone, 2006b). Since Methanosarcina was detected in sulfate-free SZ and MZ slurries, it constituted close relationships with incomplete propionate converters, and carried out acetate consumption (Fig 6, Fig S6). Methanococcoides (ANME-3 Archaea) increased its relative abundance in the SMTZ and MZ slurries (Fig 6). Lösekann et al. (2007) reported that ANME-3 live syntrophically with Desulfobulbus spp., which were present in the slurries that the relative abundance of Methanococcoides increased (Fig 4). The other anaerobic methanotrophic group that increased its relative abundance was ANME-1 (Fig 6). Most probably, anaerobic oxidation of methane occurred not only in the slurries comprised of SMTZ sediment, which was considered as the most common ANME habitat on earth (Thomsen et al., 2001; Niemann et al., 2006; Parkes et al., 2007), but also in SZ and MZ sediment slurries.

Non-methanogenic Archaea detected in the slurries included the MBG-D and DHVEG-1 belonging to Euryarchaeota and MCG belonging to Thaumarchaeota (Fig 6). These groups were previously reported as dominant archaeal groups in deep subsurface sediments in addition to coastal marine surface sediments and that their relative abundance is independent from the major biogeochemical zones, indicating their diverse metabolism (Fry et al., 20o8; Teske and Sørenson, 2008; Lloyd et al., 2013; Parkes et al., 2014; Roussel et al., 2009). Webster et al. (2010) detected MCG and MBG-D in sulfate zone sediment slurry enrichments within the active archaeal community incorporating ${ }^{13} \mathrm{C}$-acetate. As a result, MBG-D were stimulated in acetate-amended slurries. These findings are in line with our observations where both of MCG and MBG-D increased in relative 
abundances in slurries containing sulfate and their relative abundances were positively correlated with sulfate and acetate concentrations (Fig 6, S7).

\subsection{Conclusions}

In this research, we found clear changes in the bacterial and archaeal community in the long-term enrichment slurries depending on the presence/absence of sulfate, incubation temperature and, in some cases the biogeochemical zone. Propionate was converted by both sulfate-reducing and syntrophic communities in the presence of sulfate at all depth zones. The presence of defined microbial communities indicates that syntrophs are not simply out-competed by high sulfidogenic activity, rather they can stay in a dormant state with a low metabolic activity and take part in the conversion as they constitute close relationship with partner organism(s). Members of Desulfobacteraceae and Desulfobulbaceae were observed in sulfate-amended slurries as well as in sulfate-free slurries at low temperature. This indicates that changing to a syntrophic lifestyle allows sulfate reducers to remain in sulfate-depleted/limited environments and this explains the high relative abundance of SRB in deep marine sediments. The dominance of Cryptanaerobacter, without a known propionate-converting metabolism, in sulfate-free slurries at high temperature throughout the sediment brought the possibility that yet uncultured species of Cryptanaerobacter, in addition to Pelotomaculum sp., utilize propionate as a substrate in syntrophy with hydrogenotrophic methanogens. The dominant syntrophic partners belonged to the hydrogenotrophic methanogenic order Methanomicrobiales that enriched in all slurries with different representative genera and unclassified group under different incubation conditions. The Methanosarcina enriched only in the absence of sulfate, suggesting a potential competition with acetate-degrading sulfate reducers. Further cultivation studies are currently ongoing to identify the uncultured microorganisms involved in propionate conversion as well as to determine their interaction with other bacterial and archaeal groups during conversion process.

\section{Acknowledgements}

We gratefully acknowledge Mark A. Lever (Eidgenössische Technische Hochschule, Zurich, Switzerland), Kasper U. Kjeldsen, Hans Røy (Aarhus University, Aarhus, Denmark) for skillful technical assistance and the captains and crews of sailing vessel Marieje and research vessel Tyra for their help during different cruises. We thank Peer Timmers (Wageningen University \& 
Research), Martin Liebensteiner and Kivanc Ozuolmez for assistance during sampling, Ton van Gelder (Wageningen University \& Research) for technical assistance in the laboratory. This work has been funded by the Wimek Graduate School of Wageningen University \& Research and the Darwin Center for Biogeosciences (the Netherlands). Research of Alfons Stams is supported by ERC grant (project 323009) Gravitation grant (project 024.002.002) of the Netherlands Ministry of Education, Culture and Science and the Netherlands Science Foundation (NWO). 


\section{Supplementary data}

Table S1. The overview of all the enrichment slurries fed with propionate and the total amounts of the reactants consumed and products formed during the enrichment period. The enrichment slurries were consisted of sediment either from sulfate zone (SZ), sulfate-methane transition zone (SMTZ) or methane zone (MZ) and incubated at $25^{\circ} \mathrm{C}$ or $10^{\circ} \mathrm{C}$, with $3 \mathrm{mM}, 20 \mathrm{mM}$ or without (-) sulfate amendments along the study. Slurries with * were presented in the propionate conversion graphs and used for molecular analysis.

\begin{tabular}{|c|c|c|c|c|c|c|c|c|}
\hline \multirow{2}{*}{ Sediment zone } & \multirow{2}{*}{ Slurry Code } & \multirow{2}{*}{ Treatment } & \multirow{2}{*}{$\begin{array}{c}\text { Incubation } \\
\text { temperature }\left({ }^{\circ} \mathrm{C}\right)\end{array}$} & \multicolumn{2}{|c|}{ Reactants ( $\mu \mathrm{mol} / \mathrm{s}$ lurry) } & \multicolumn{3}{|c|}{ Products ( $\mu \mathrm{mol} / \mathrm{s}$ lurry) } \\
\hline & & & & Propionate & Sulfate & Acetate & Sulfide & Methane \\
\hline \multirow{8}{*}{ sz } & *P1 & - & 25 & 26157 & 336 & 17905 & 881 & 37464 \\
\hline & P2 & - & 25 & 2726 & 159 & 1183 & 284 & 0 \\
\hline & P3 & $20 \mathrm{mM} \mathrm{SO}_{4}{ }^{2-}$ & 25 & 20623 & 29592 & 19126 & 29062 & 33 \\
\hline & *P4 & $20 \mathrm{mM} \mathrm{SO}_{4}^{2-}$ & 25 & 26681 & 42456 & 25677 & 39089 & 1038 \\
\hline & P5 & - & 10 & 17190 & 354 & 13170 & 151 & 9083 \\
\hline & *P6 & - & 10 & 18414 & 125 & 9558 & 207 & 14959 \\
\hline & P7 & $20 \mathrm{mM} \mathrm{SO}_{4}^{2-}$ & 10 & 15491 & 13410 & 18442 & 18296 & 0 \\
\hline & *P8 & $20 \mathrm{mM} \mathrm{SO}_{4}^{2-}$ & 10 & 17679 & 18592 & 21716 & 21495 & 0 \\
\hline \multirow{8}{*}{ SMTZ } & P1 & $3 \mathrm{mM} \mathrm{SO}_{4}{ }^{2-}$ & 25 & 27494 & 9343 & 25160 & 6993 & 29829 \\
\hline & *P2 & $3 \mathrm{mM} \mathrm{SO}_{4}{ }^{2-}$ & 25 & 32207 & 9330 & 34093 & 8892 & 15509 \\
\hline & *P3 & $20 \mathrm{mM} \mathrm{SO}_{4}{ }^{2-}$ & 25 & 27819 & 37381 & 9246 & 34439 & 339 \\
\hline & P4 & $20 \mathrm{mM} \mathrm{SO}_{4}{ }^{2-}$ & 25 & 28694 & 40125 & 11250 & 35587 & 74 \\
\hline & *P5 & $3 \mathrm{mM} \mathrm{SO}_{4}^{2-}$ & 10 & 18465 & 6638 & 16996 & 7316 & 2797 \\
\hline & P6 & $3 \mathrm{mM} \mathrm{SO}_{4}{ }^{2-}$ & 10 & 17365 & 6839 & 17427 & 7642 & 2197 \\
\hline & *P7 & $20 \mathrm{mM} \mathrm{SO}_{4}{ }^{2-}$ & 10 & 26008 & 33660 & 27709 & 34299 & 17 \\
\hline & P8 & $20 \mathrm{mM} \mathrm{SO}_{4}{ }^{2-}$ & 10 & 27162 & 35346 & 26523 & 36833 & 34 \\
\hline \multirow{8}{*}{ MZ } & *P1 & - & 10 & 7204 & 932 & 7346 & 552 & 3909 \\
\hline & P2 & - & 10 & 3897 & 809 & 1575 & 673 & 926 \\
\hline & *P3 & - & 25 & 27472 & 825 & 13791 & 563 & 38473 \\
\hline & P4 & - & 25 & 34576 & 604 & 20771 & 509 & 43969 \\
\hline & P5 & $20 \mathrm{mM} \mathrm{SO}_{4}^{2-}$ & 25 & 39414 & 33244 & 30817 & 32821 & 1670 \\
\hline & *P6 & $20 \mathrm{mM} \mathrm{SO}_{4}{ }^{2-}$ & 25 & 49211 & 45738 & 49783 & 40572 & 418 \\
\hline & P7 & $20 \mathrm{mM} \mathrm{SO}_{4}{ }^{2-}$ & 10 & 16577 & 20444 & 15542 & 15184 & 0 \\
\hline & *P8 & $20 \mathrm{mM} \mathrm{SO}_{4}^{2-}$ & 10 & 11366 & 16233 & 11913 & 12356 & 0 \\
\hline
\end{tabular}


Table S2. The number of reads per sample generated by pyrosequencing for Bacteria and HiSeq Illumina sequencing for Archaea.

\begin{tabular}{cccc}
\hline Origin & Slurry & Bacterial reads & Archaeal reads \\
\hline \multirow{4}{*}{ Sulfate zone } & ENV & 8733 & 9120 \\
& P1 & 9903 & 13481 \\
& P4 & 12305 & 74345 \\
& P6 & 27196 & 67941 \\
& P8 & 15062 & 16472 \\
\hline \multirow{4}{*}{ Sulfate-methane } & ENV & 3186 & 18696 \\
& P2 & 8730 & 33641 \\
& P3 & 5653 & 30858 \\
& P5 & 5949 & 79958 \\
& P7 & 3934 & 21631 \\
\hline \multirow{3}{*}{ Methane zone } & ENV & 10824 & 30848 \\
& P1 & 14192 & 78094 \\
& P3 & 14752 & 108810 \\
& P6 & 14171 & 25156 \\
& P8 & 1888 & 5547 \\
\hline
\end{tabular}

Figure S1. Depth profiles of sediment pore water sulfate, sulfide and methane for Station M1, in Aarhus Bay, Denmark. Methane-GC1 and Methane-GC2 stands for methane concentrations retrieved from two different gravity corers, gravity corer 1 and 2, respectively. SZ; Sulfate zone, SMTZ; sulfate-methane transition zone; MZ, methane zone.

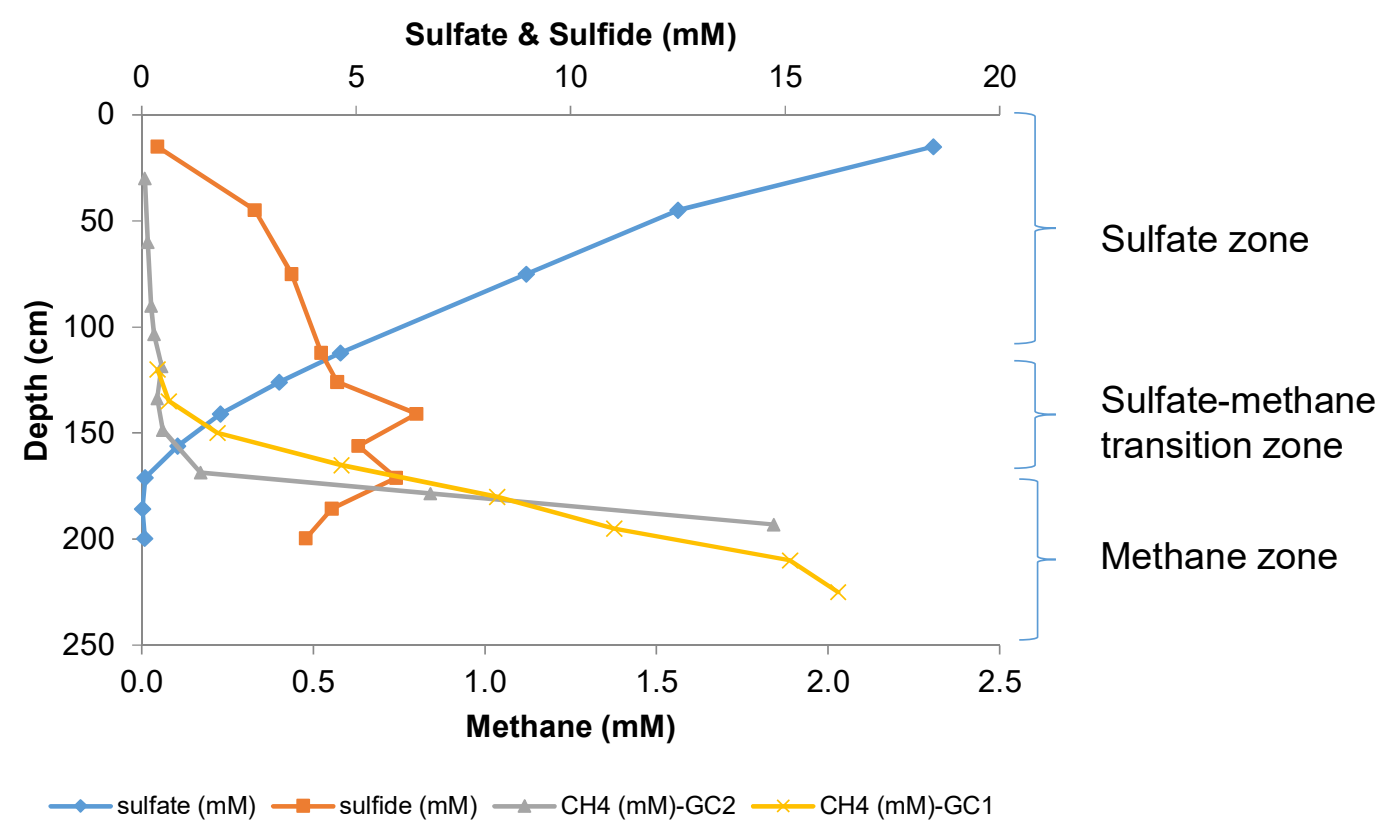




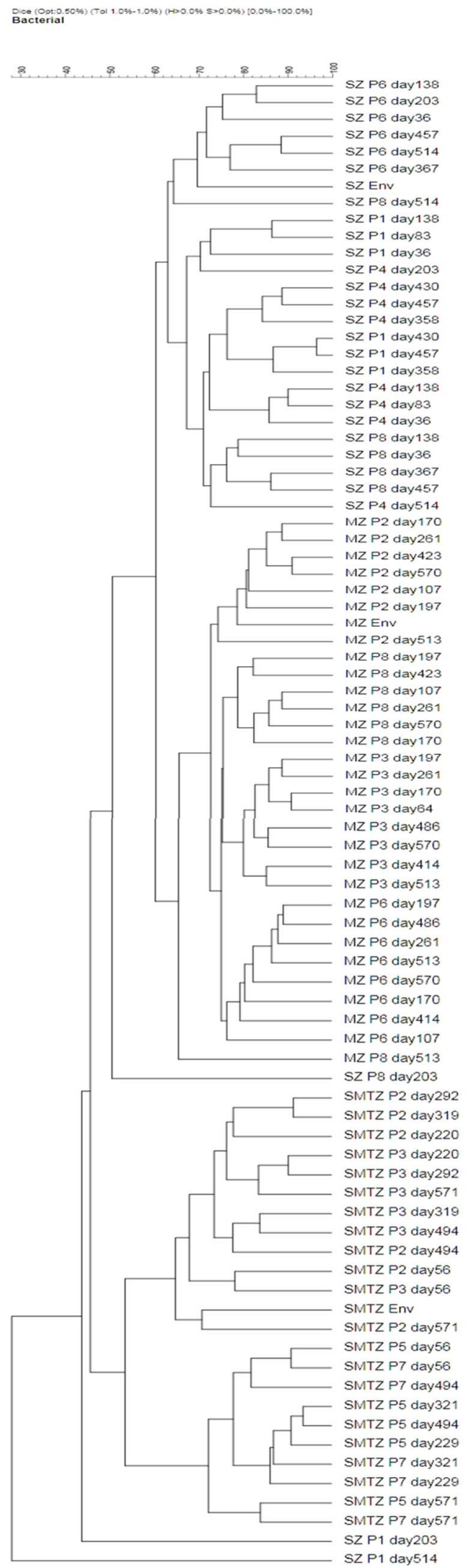

Figure S2. Cluster analysis of bacterial DGGE profiles of sediment samples taken at different biogeochemical zones and samples of enrichment slurries that were taken at different time points. The trees were generated using Dice similarity coefficient and UPGMA clustering algorithm. SZ: Sulfate zone, SMTZ: Sulfatemethane transition zone, MZ: Methane zone. 


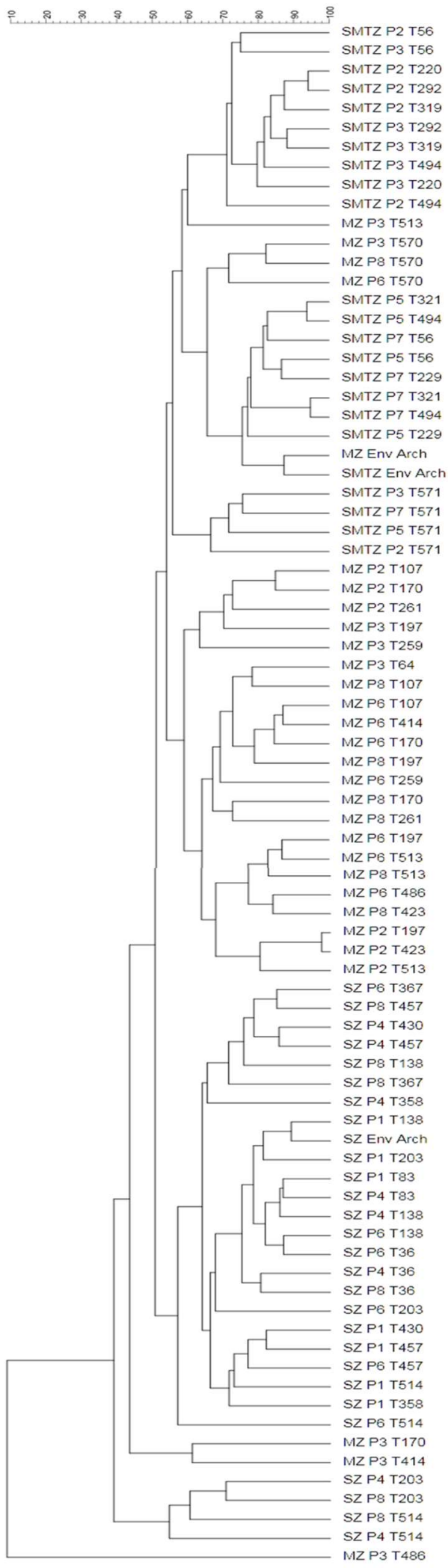

Figure S3. Cluster analysis of archaeal DGGE profiles of sediment samples taken at different biogeochemical zones and samples of enrichment slurries that were taken at different time points. The trees were generated using Dice similarity coefficient and UPGMA clustering algorithm. SZ: Sulfate zone, SMTZ: Sulfatemethane transition zone, MZ: Methane zone. 
Figure S4. Relative abundances of the bacterial community in all slurries and environmental samples at family level, normalized to $100 \%$. Only those families that were present at an abundance $>1 \%$ in at least one sample were included in the graph. SZ: Sulfate zone, SMTZ: Sulfate-methane transition zone; MZ: Methane zone. Env: Sediment sample belonging to the indicated biogeochemical zone. S: $20 \mathrm{mM}$ sulfate, $3 \mathrm{~S}: 3 \mathrm{mM}$ sulfate is used as electron acceptor in slurries. Slurries that were not labeled with 'S' or ' $3 \mathrm{~S}$ ' were incubated without sulfate. The number of reads of each sample was given in brackets.

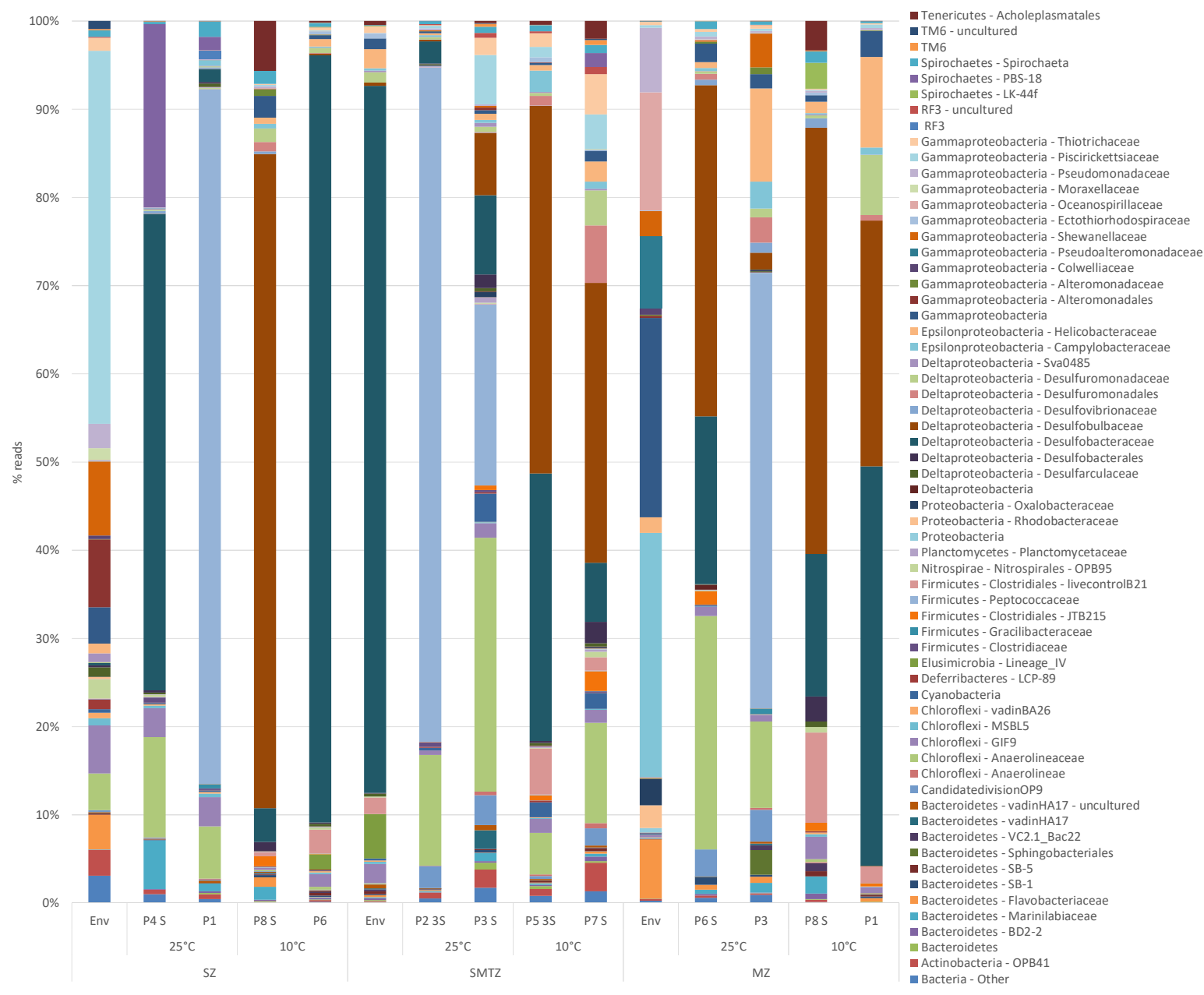


Figure S5. The heatmap depicts the correlation between bacterial orders present at a relative abundance $>1 \%$ of total reads across the 12 slurry samples analyzed and experimental parameters. Correlations were determined by means of the two tailed Spearman's Rank Order Correlation test. The heatmap colors represent the relative percentage of the microbial order assignments. Square colors shifted towards bright green indicate strong correlation.

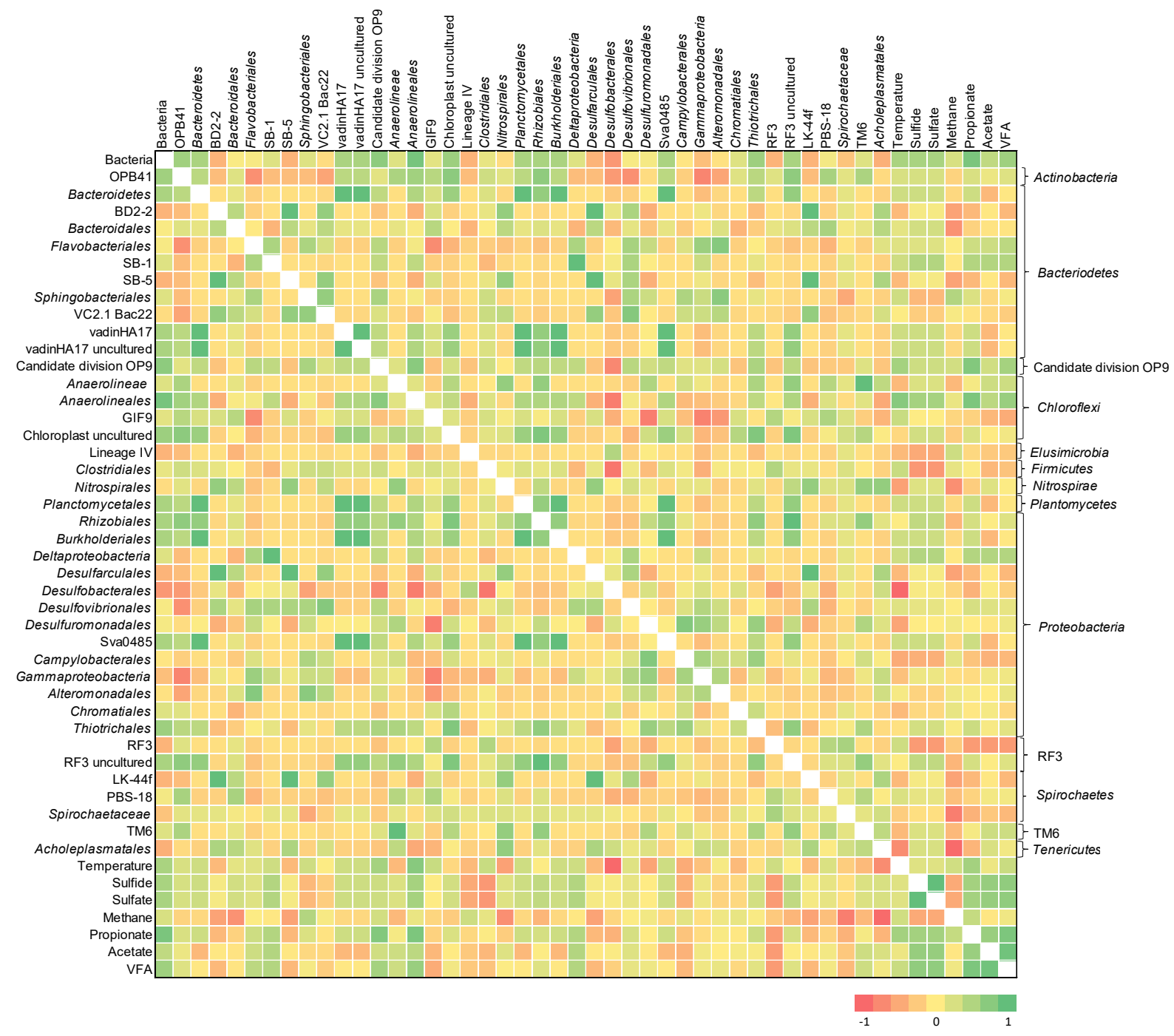


Figure S6. Relative abundances of the archaeal community in all slurries and environmental samples at family level, normalized to $100 \%$. Only those families that were present at an abundance $>1 \%$ in at least one sample were included in the graph. SZ: Sulfate zone, SMTZ: Sulfate-methane transition zone; MZ: Methane zone. Env: Sediment sample belonging to the indicated biogeochemical zone. S: $20 \mathrm{mM}$ sulfate, $3 \mathrm{~S}: 3 \mathrm{mM}$ sulfate is used as electron acceptor in slurries. Slurries that were not labeled with 'S' or ' $3 S^{\prime}$ ' were incubated without sulfate.

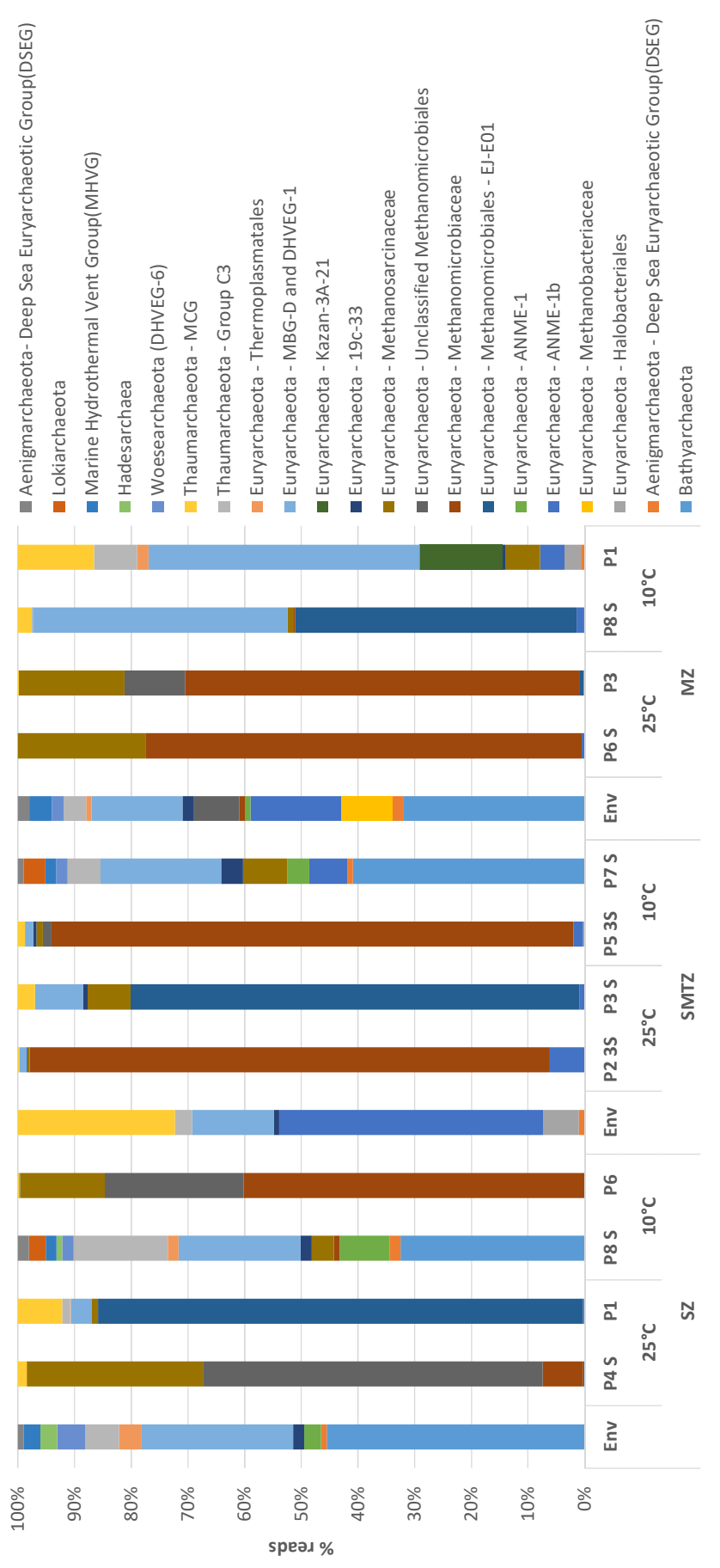


Figure S7. The heatmap depicts the correlation between archaeal families present at a relative abundance $>\mathbf{1} \%$ of total reads across the 12 slurry samples analyzed and experimental parameters. Correlations were determined by means of the two tailed Spearman's Rank Order Correlation test. The heatmap colors represent the relative percentage of the microbial family assignments. Square colors shifted towards bright green indicate strong correlation.

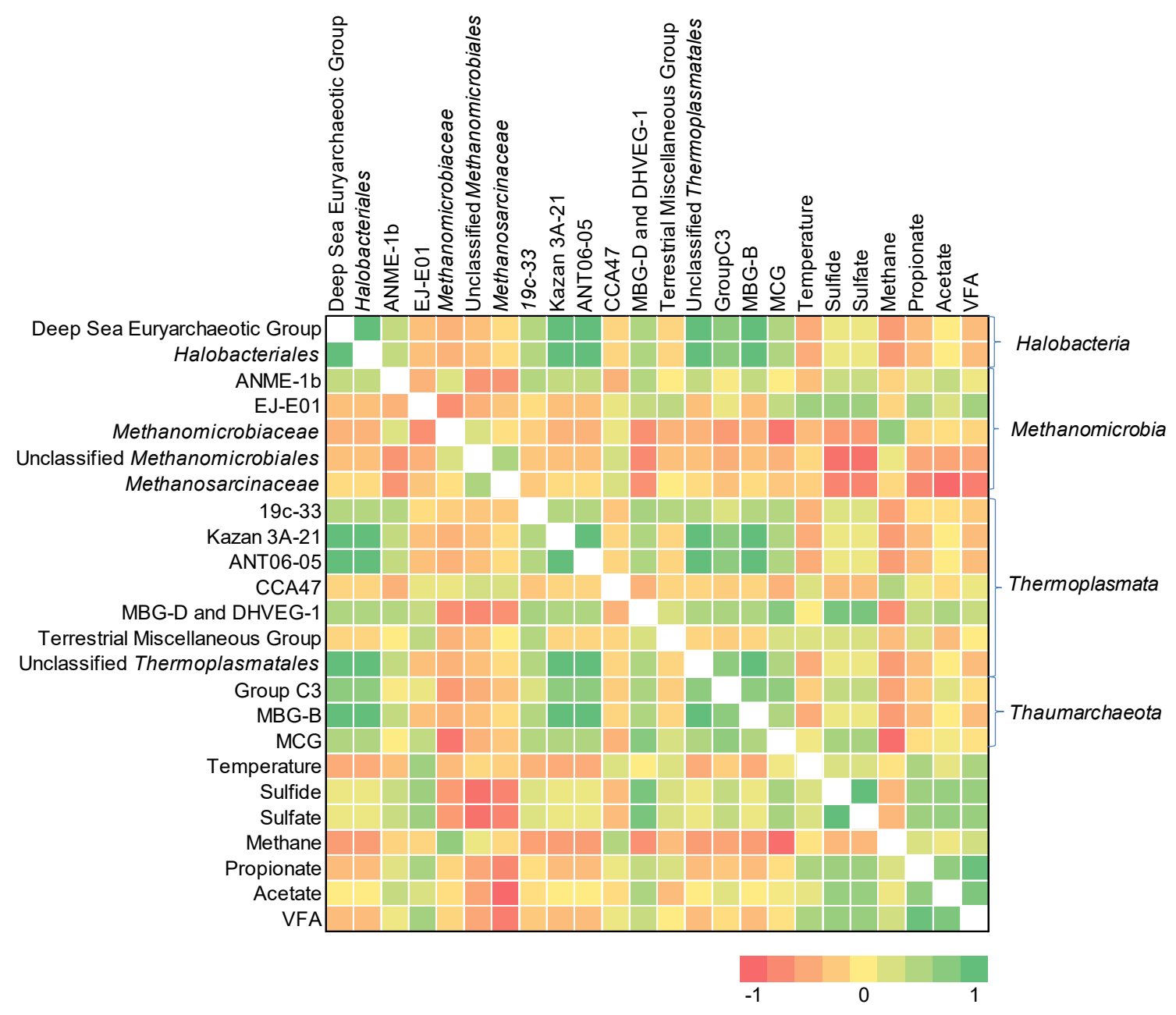


Chapter 4 


\section{Chapter 5}

\section{Membrane lipid composition in enrichments from the sulfate and methane zones of Aarhus Bay, Denmark}

Derya Ozuolmez ${ }^{*}$, Eli K. Moore*, Ellen C. Hopmans, Caroline M. Plugge, Jaap S. Sinninghe Damsté $(*)$ Contributed equally 


\section{Abstract}

Sulfate reduction and methanogenesis are important terminal electron accepting processes contributing to the organic matter $(\mathrm{OM})$ degradation under anoxic conditions in coastal marine sediments. It is not well known which metabolic strategies are used by sulfate reducing bacteria (SRB) in the methane zone and methanogenic archaea in the sulfate zone, or which physiological adaptations are utilized under different metabolic regimes. To understand how the microbial community responds to different growth conditions and substrates, enrichment slurries were developed using sediment taken from sulfate, sulfate-methane transition and methane zones of Aarhus Bay, Denmark. Enrichment slurries were amended with different sulfate concentrations (o, 3, $20 \mathrm{mM}$ ) and carbon substrates (propionate and butyrate) and were incubated at two different temperatures $\left(10^{\circ} \mathrm{C}\right.$ and $\left.25^{\circ} \mathrm{C}\right)$. Intact polar membrane lipid (IPL) analysis and next generation sequencing of the 16S rRNA gene were performed on the enrichment slurries at the end of the incubation period. Overall, the distribution of IPLs and microbial community shifted between different sediment zones and growth conditions, with greater IPL diversity found in butyrateamended cultures as compared to the propionate-amended enrichment slurries. Recently discovered trimethylornithine lipids (TMOs) were identified in three butyrate-amended methane zone slurries. The presence of TMO lipids in these slurries could be linked with the enrichment of methanogenic microbial communities, as TMOs were observed only in methanogenic environments to date. Multivariate analysis showed that Bacteroidetes and Methanomicrobiales taxa, and sulfate amendments were closely clustered with many IPLs indicating that these taxa contributed to the IPL variation among the enrichment slurries. 


\subsection{Introduction}

Organic matter (OM) degradation in coastal marine sediments is an important process in the global carbon cycle (Henrichs, 1992; Hedges and Keil, 1995; Burdige, 2007). In oxic sediments, OM is degraded ultimately to carbon dioxide and water, whereas under anoxic conditions organic matter is degraded by physiologically different microorganisms employing fermentation or terminal electron accepting processes. In marine sediments, where sulfate concentration is high, sulfate reduction is the predominant pathway for OM degradation, while methanogenesis becomes important in zones where sulfate is low or depleted (Jørgensen, 1982; Holmkvist et al., 2011; Bowles et al., 2014). Yet the diversity and metabolic capabilities of marine anaerobic microbes are largely unexplored (Jørgensen and Boetius, 2007). The characterization of microbial communities involved in sulfate reduction and methanogenesis is essential to understand OM decomposition in coastal marine sediments.

Anaerobic degradation of organic matter in marine sediments is a complex process involving physiologically different microorganisms (Schink and Stams, 2013). The first step is an extracellular hydrolytic conversion of polymers to oligomers and monomers which is followed by fermentation of these compounds to reduced organic compounds such as short chain fatty acids, alcohols, formate, $\mathrm{H}_{2}$ and $\mathrm{CO}_{2}$. Organic acids and alcohols are further degraded to acetate, formate, $\mathrm{H}_{2}$ and $\mathrm{CO}_{2}$. When sulfate concentration is high, SRB can use all fermentation products, and oxidize them to $\mathrm{CO}_{2}$ by reducing sulfate to sulfide (Schink and Stams, 2013). Sulfate-depleted marine sediments are major sources of biogenic methane. In the absence of sulfate, complex organic material is mineralized exclusively to $\mathrm{CO}_{2}$ and methane by physiologically diverse microorganisms that are co-operating, including fermentative, acetogenic bacteria, and methanogenic archaea (de Bok et al., 2005; Stams et al., 2005; Dolfing et al., 2008; Plugge et al., 2009; Müller et al., 2010). Propionate and butyrate are important intermediates in anaerobic methanogenic food chain since their complete conversion can be accomplished only by syntrophically (Schink and Stams, 2013; McInerney et al., 2008). The studies showing the presence of sulfate reducers in the methane and methanogens in sulfate zones of marine sediments indicates the importance of hydrogen, that is produced during organic matter mineralization, on the metabolism of methanogenic and the sulfate-reducing microbial populations (Plugge et al., 2011; Finke et al., 2007b; Leloup et al., 2009; Leloup et al., 2007). Further research is needed to determine what type of metabolism sulfate reducers use in the methane zone, and methanogenic archaea in the sulfate zone. 
Intact polar membrane lipids (IPL) are the building blocks of cell membranes and representative of living biomass as they rapidly hydrolyze upon cell lysis (White et al., 1979; Harvey et al., 1986). IPLs can also be structurally specific to microbial taxa or may differ depending on the habitat characteristics, thus providing information of the biogeochemistry of an environment (Sturt et al., 2004; Schubotz et al., 2009). Analysis of IPLs by high performance liquid chromatographyelectrospray ionization-mass spectrometry (HPLC/ESI/MS) can provide information on the physiological status of microbial communities as the structural composition of lipid membranes is influenced by environmental conditions such as growth temperature, $\mathrm{pH}$, and nutrient limitation (Shimada et al., 2008; Van Mooy et al., 2009; Vences-Guzmán et al., 2011; Moore et al., 2015a). IPL distributions in marine sediments have also been observed to change in accordance with habitat characteristics and inhabiting microbial community (Schubotz et al., 2009; Rossel et al., 2011). In this study, enrichment slurries were established using sulfate zone (SZ), sulfate-methane transition zone (SMTZ) and methane zone (MZ) sediments of Aarhus Bay, Denmark. Propionate and butyrate were used as carbon sources, different sulfate concentrations were applied, and incubated at $10^{\circ} \mathrm{C}$ and $25^{\circ} \mathrm{C}$. IPL analysis and next generation sequencing of the $16 \mathrm{~S}$ rRNA gene were performed on the last incubation day samples of enrichment slurries and the original sediment to understand the microbial responses to different incubation conditions.

\subsection{Materials and Methods}

\subsubsection{Sediment sampling and enrichment slurry incubations}

Sediment cores were collected in May 2011 in Aarhus Bay, Denmark (56 07'066”N, $10^{\circ} 20^{\prime} 793$ "E). Subsamples representing sulfate zone (SZ, 15-120 cm), sulfate-methane transition zone $(\mathrm{SMTZ}, 120-170 \mathrm{~cm})$ and methane zone $(\mathrm{MZ}, 170-300 \mathrm{~cm})$ were mixed in an anaerobic chamber and used as inoculum for sediment slurry enrichments. $100 \mathrm{ml}$ of the homogenized sediment from each zone was mixed with $300 \mathrm{ml}$ of anaerobic mineral salts medium in $1 \mathrm{~L}$ serum bottles. Media was prepared as was described in Chapter 2. $10 \mathrm{mM}$ propionate or butyrate was used as carbon sources with and without $20 \mathrm{mM}$ sulfate in sulfate zone and methane zone slurries, and with $3 \mathrm{mM}$ and 20 $\mathrm{mM}$ sulfate for sulfate-methane transition zone slurries as electron acceptor. One set of the bottles representing each condition in duplicate was incubated at $10^{\circ} \mathrm{C}$ as in situ temperature (Dale et al., 2008) and the other set was kept at $25^{\circ} \mathrm{C}$ statically throughout the experiment. The overview of enrichment slurries and incubation conditions is given in chapters 3 and 4 . At the end of the 
enrichment period ( 514 days for SZ, 571 days for SMTZ, and 570 days for MZ), $1 \mathrm{ml}$ of liquid slurry samples were collected for IPL analysis, freeze dried and stored at $-80^{\circ} \mathrm{C}$ before IPL extraction.

\subsubsection{Intact polar lipid extraction}

The slurry samples which were used for molecular analysis were used for lipid analysis. Additionally, methane zone slurry samples which were not analysed for molecular analysis were also extracted and analyzed in order to confirm the presence or absence of trimethylornithine lipids (TMOs). Lipids were extracted from $\sim 0.1 \mathrm{~g}$ freeze-dried powdered enrichment cultures using a method adapted from Bligh and Dyer (1959) (Rütters et al., 2002a). A solvent mixture (approximately $5 \mathrm{ml} \mathrm{g}^{-1}$ dry weight, $\mathrm{dw}$ of methanol $(\mathrm{MeOH})$ :dichloromethane (DCM): potassium phosphate buffer at $\mathrm{pH} 7.4(2: 1: 0.8, \mathrm{v} / \mathrm{v} / \mathrm{v})$ was added to approximately $0.3^{-1.3} \mathrm{~g}$ dry weight of powdered enrichment culture in a centrifuge tube and placed in an ultrasonic bath for $10 \mathrm{~min}$. After sonication, the powdered enrichment culture-solvent mixture was centrifuged at 2,500 rpm for 2 min and the overlying solvent extract was pipetted off. The extraction was repeated twice and the replicate extracts were combined with the first extract for each sample. DCM and phosphate buffer were added to the combined extracts to yield a ratio of 1:1:0.9 (v/v/v) and achieve separation of a DCM phase and an aqueous $\mathrm{MeOH} /$ phosphate buffer phase by centrifugation at 2,500 rpm for 2 min. The DCM phase, containing the IPLs, was pipetted off and passed over extracted cotton wool to remove any remaining particles and collected in a glass tube. The aqueous phase was rinsed twice with DCM, and each DCM rinse also passed over extracted cotton wool and combined with the original DCM phase. The combined DCM samples were dried under a $\mathrm{N}_{2}$ flow and stored at $-20^{\circ} \mathrm{C}$ until analysis.

\subsubsection{HPLC-ESI/IT/MS analysis of IPLS}

Extracted IPLs from the sediment enrichment slurries were analyzed by high-performance liquid chromatography-electrospray ionization-ion trap mass spectrometry (HPLC-ESI/IT/MS) according to Sturt et al. (2004), with some modifications (Moore et al., 2013). An Agilent 1200 series highperformance liquid chromatograph (Agilent, San Jose, CA), with thermostatted autoinjector was coupled to a Thermo LTQ XL linear ion trap mass spectrometer with an Ion Max source and ESI probe (Thermo Scientific, Waltham, MA). Chromatographic separation was performed on a Lichrosphere diol column (250 mm by $2.1 \mathrm{~mm}$; 5 - $\mu \mathrm{m}$ particles; Grace Alltech Associates Inc., 
Deerfield, IL). The MS scanning mass range of $\mathrm{m} / \mathrm{z} 400$ to 2,000 in positive-ion mode, followed by data dependent dual-stage tandem MS $\left(\mathrm{MS}^{2}\right)$, in which the four most abundant masses in the mass spectrum were fragmented successively. Each $\mathrm{MS}^{2}$ was followed by data-dependent, triple-stage tandem MS (MS3), wherein the base peak of the MS² spectrum was fragmented. IPL abundance was assessed by integrating the HPLC-ESI/IT/MS base peak chromatogram area per gram of peat, dry weight. Performance of the HPLC-ESI/IT/MS was monitored by regular injections of plateletactivating factor (PAF) standard (1-O-hexadecyl-2-acetyl-sn-glycero-3-phosphocholine).

\subsubsection{Statistical analyses}

Multivariate canonical correspondence analysis (CCA) (McGarigal et al., 200o) was performed to compare the microbial taxa identified in chapters 3 and 4 with the associated distribution of IPLs in each enrichment slurry in order to find phylogenetic groups and IPLs which could correlate with each other or with particular incubation conditions. CCA was executed using R statistical analysis software.

\subsection{Results}

\subsubsection{Microbial community analysis}

The microbial diversity varied much between different sediment zones and different incubation conditions as described in chapters 3 and 4 . These results are described here briefly for comparison with IPL results. Bacterial taxa Thiomicrospira, GIF9 from the phylum Chloroflexi, and Arcobacter were the most abundant microbial taxa in sediment of the SZ, SMTZ and MZ, respectively. As compared to the original sediment, all three taxa were relatively minor in abundance in both propionate- and butyrate-amended sediment slurries which originated from the corresponding sediment zones. The microbial composition in enrichment slurries representing each sediment zone changed considerably under different incubation conditions. Cryptanaerobacter belonging to Peptococcaceae was the most abundant phylotype in low or no sulfate-containing propionateamended enrichment slurries from each sediment zone at $25^{\circ} \mathrm{C}$. In butyrate-amended sediment slurries, Anaerolineaceae was abundant at $25^{\circ} \mathrm{C}$ under both high and low sulfate conditions. Other taxa were highly abundant only in one particular enrichment slurry, such as Desulfobacteraceae in the $10^{\circ} \mathrm{C}$ low sulfate propionate-amended sulfate zone slurry, or Desulfobacterium in the $10^{\circ} \mathrm{C}$ high sulfate butyrate-amended sulfate zone slurry. Sulfate zone sediment slurries were dominated by 
one phylotype. Five out of eight SZ slurries contained one phylotype accounting for over 50\% relative abundance and two other slurries contained one taxa accounting for over $45 \%$ relative abundance. Unassigned taxa were generally low in relative abundance.

The archaeal taxa were not as diverse as the bacterial taxa, and it was more common for one phylogenetic group to make up a large majority of the total Archaea than for Bacteria. In propionate-amended sediment slurries, uncultured Methanomicrobiales (EJ-Eo1) was highly abundant in $25^{\circ} \mathrm{C}$ high sulfate slurries of each sediment zone, and Methanomicrobiaceae was highly abundant in all low/no sulfate slurries of each sediment zone at both $10^{\circ} \mathrm{C}$ and $25^{\circ} \mathrm{C}$. In propionateamended sulfate-containing methane zone slurries, MBG-D and DHVEG-1 belonging to Thermoplasmatales was highly abundant. Methanomicrobiaceae was the most abundant taxon in all butyrate-amended sediment slurries except for the SMTZ slurries incubated with high sulfate at $10^{\circ} \mathrm{C}$ and with low sulfate at $25^{\circ} \mathrm{C}$. Methanosarcinaceae was the second most abundant archaeal taxon in butyrate-amended slurries with Methanosaetaceae having high relative abundance in two slurries.

The overall diversity was greater for bacterial and archaeal taxa compared to the diversity of IPL head group classes. There were 380 different bacterial and 17 archaeal taxa identified in propionate-amended sediment slurries, versus 7 different IPL head group classes and 29 different IPL head group core lipid combinations identified in the propionate-amended sediment slurries. Similarly, there were 384 bacterial and 17 archaeal taxa identified in butyrate-amended sediment slurries, versus 14 different IPL head group classes and 55 different IPL head group core lipid combinations identified in the butyrate-amended sediment slurries.

\subsubsection{Dominant IPLs in enrichment slurries and original sediment}

The most abundant classes of IPLs identified in most of the sediment enrichment slurries were phosphatidylethanolamine (PE), phosphatidylglycerol (PG), phosphatidylcholine (PC), lyso-PC (LPC; PC containing one fatty acid), and dihexose (DH) containing lipids (Fig. 1, 2, Table S1). Other identified IPLs (MH, PA, OL, TMO, PI, DMPE, Betaine, MMPE, GlcA) were found in some enrichment slurries, although some had relatively high abundances in certain slurries, such as monomethylphosphatidylethanolamine (MMPE) in slurry SZP8 (30425552 peak area/g dry weight) or glucuronic acid (GlcA) in culture SMTZB1 (35097613 peak area/g dry weight). Tetraether lipids, which are known to be attributed to archaea (De Rosa and Gambacorta, 1988; Kates, 1993; Koga et al., 1993; Hoefs et al., 1997; Hopmans et al., 2000), were not detected in the sediment slurries. IPL 
abundance (per gram dry weight) and diversity were much lower in the original sediment core extracts compared to the enrichment slurries (Fig. 1, 2, Table S1). The IPLs identified in the original sediment extracts were PA, PE, and phosphatidylinositol (PI) with PA and PE being identified in all sediment zones analyzed, and PI was only identified in the SZ (Table S1). The IPL distributions and microbial communities of the enrichment slurries composed of various biogeochemical zones (SZ, SMTZ, MZ) responded differently to different incubation conditions, particularly when they are compared to the IPL distributions and microbial communities in the corresponding sediment zones.

\subsubsection{IPL distributions in enrichment slurries}

\subsubsection{Sulfate zone enrichment slurries}

PE was identified in all sulfate zone enrichment slurries occurring in moderately high abundances (between 2125058-11455795 peak area/g dry weight) in two $10^{\circ} \mathrm{C}$ cultures amended with different substrates and sulfate concentrations (Fig. 1, 2, Table S1). PG was detected in $10^{\circ} \mathrm{C}$ butyrateamended slurries in the presence of sulfate and $10^{\circ} \mathrm{C}$ propionate-amended slurries in the presence and absence of sulfate. In propionate-amended sulfate zone slurries, DH was observed only in the absence of sulfate at both temperatures, whereas in butyrate-amended sulfate zone slurries DH occurred both in the absence of sulfate at both temperatures and in the presence of sulfate at $25^{\circ} \mathrm{C}$. PC was detected in two butyrate-amended slurries in the presence of sulfate at both temperatures, and LPC was detected in all but one of the sulfate containing slurries amended with butyrate and incubated at $10^{\circ} \mathrm{C}$. MMPE was detected only in one slurry which was fed with propionate and sulfate and incubated at $10^{\circ} \mathrm{C}$. 


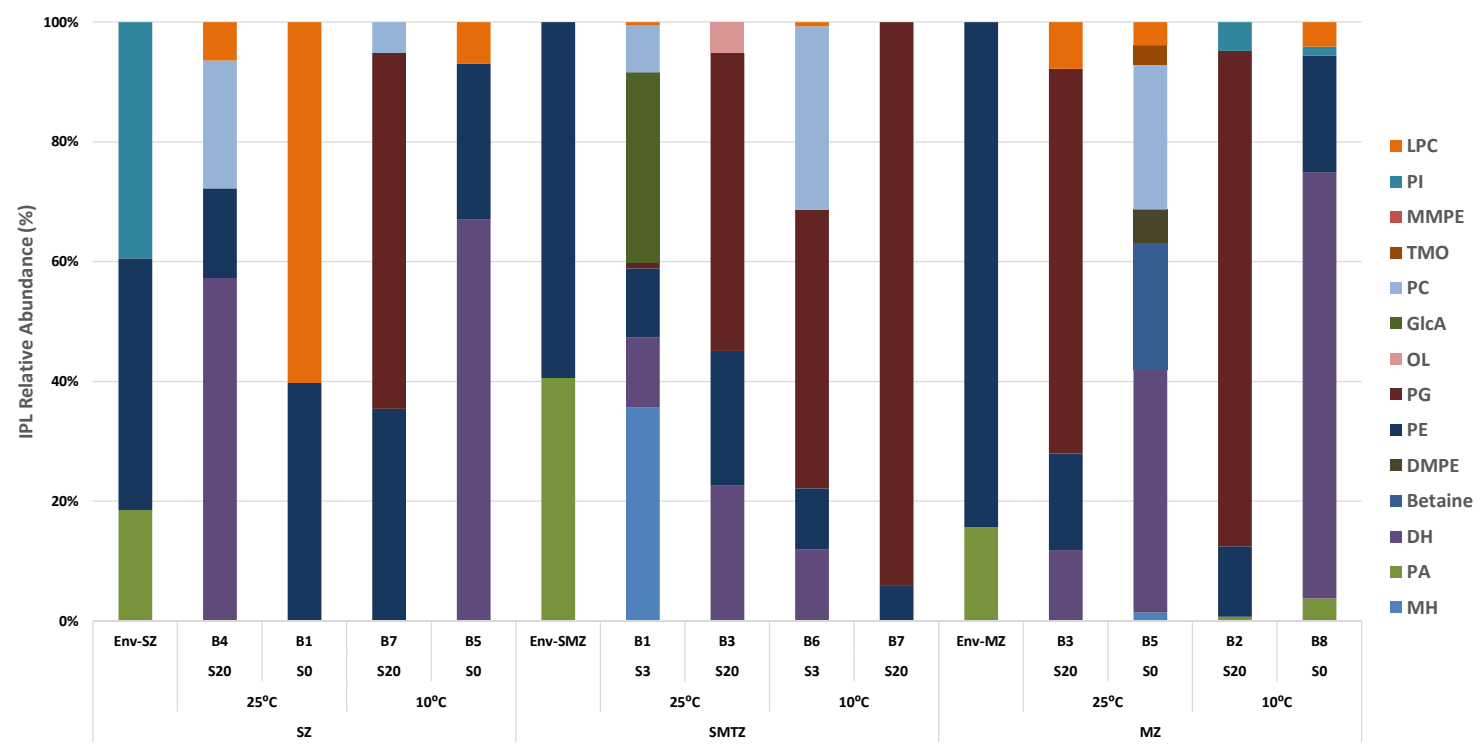

Figure 1: Relative abundance of intact polar lipids (IPLs) in butyrate-amended enrichment slurries. Env = environmental samples, B = slurry code (listed in Table $S_{1}$ in chapters 3 and 4), $S_{20}=20 \mathrm{mM}$ sulfate added, So $=0 \mathrm{mM}$ sulfate added, $\mathrm{S}_{3}=3 \mathrm{mM}$ sulfate added, ${ }^{\circ} \mathrm{C}$ indicates incubation temperature, $\mathrm{SZ}=$ sulfate zone, $\mathrm{SMTZ}=$ sulfate-methane transition zone, $\mathrm{MZ}=$ methane zone. IPL key: $\mathrm{MH}=$ monohexose, $\mathrm{PA}=$ phosphatidic acid, $\mathrm{DH}=$ dihexose, $\mathrm{DMPE}=$ dimethylphosphatidylethanolamine, $\mathrm{PE}=$ phosphatidylethanolamine, $\mathrm{PG}=$ phosphatidylglycerol, $\mathrm{OL}=$ ornithine, GlcA =glucuronic acid, $\mathrm{PC}=$ phosphatidylcholine, $\mathrm{TMO}=$ trimethylornithine, MMPE = monomethylphosphatidylethanolamine, PI = phosphatidylinositol, LPC = lysophosphatidylcholine.

\subsubsection{Sulfate-methane transition zone slurries}

PG was identified in all SMTZ slurries except for the one that contained high sulfate and propionate at $25^{\circ} \mathrm{C}$ and had higher abundance in butyrate-amended slurries (22801451 peak area/g dry weight on average) than propionate-amended slurries (4355003 peak area/g dry weight on average) (Figs. 1, 2, Table S1). PE occurred in all SMTZ slurries except for the $10^{\circ} \mathrm{C}$ low sulfate propionate-amended slurry and higher PE abundances were detected on average in butyrate-amended slurries. DH was identified in one propionate-amended slurry $\left(25^{\circ} \mathrm{C}\right.$, low sulfate) and three butyrate-amended slurries, with higher abundance than in the propionate-amended slurry. PC was detected only in three butyrate-amended slurries with higher abundance in two $10^{\circ} \mathrm{C}$ slurries, and LPC was detected in one propionate- and two butyrate-amended slurries all at low abundances (520065-711956 peak area/g dry weight). PI was identified only in three propionate-amended slurries having different sulfate concentrations and incubated at different temperatures. Monohexose ( $\mathrm{MH})$, GlcA, and 
ornithine lipid (OL) were all detected in two butyrate-amended slurries incubated at $25^{\circ} \mathrm{C}$, with $\mathrm{MH}$ and GlcA both at low and high sulfate and OL only under high sulfate conditions.

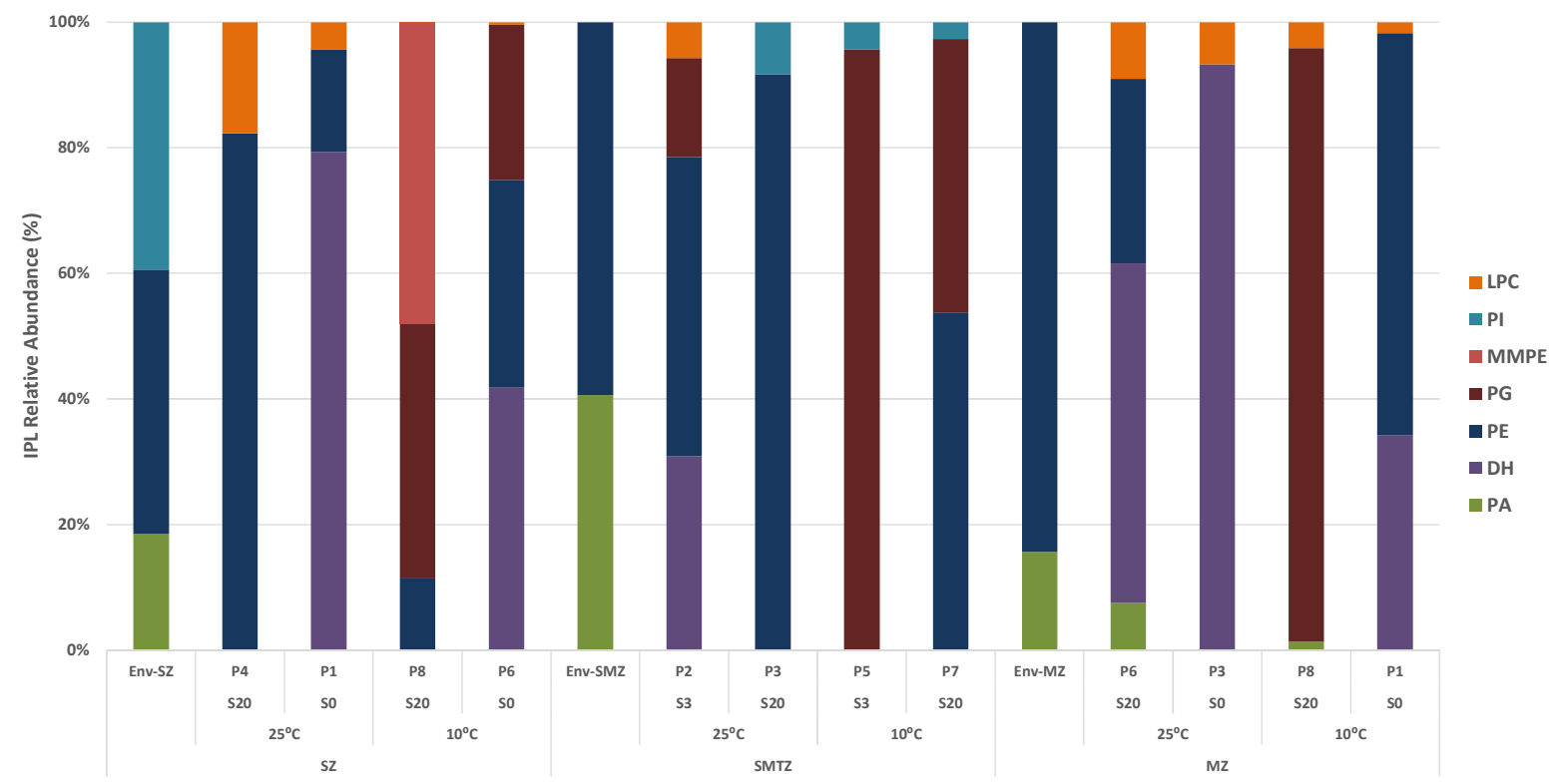

Figure 2: Relative abundance of intact polar lipids (IPLs) in propionate-amended enrichment slurries. Env = environmental samples, B = slurry code (listed in Table S1 in chapters 3 and 4), S2o $=20 \mathrm{mM}$ sulfate added, $\mathrm{So}=\mathrm{o} \mathrm{mM}$ sulfate added, $\mathrm{S}_{3}=3 \mathrm{mM}$ sulfate added, ${ }^{\circ} \mathrm{C}$ indicates incubation temperature, $\mathrm{SZ}$ = sulfate zone, $\mathrm{SMTZ}=$ sulfate-methane transition zone, $\mathrm{MZ}=$ methane zone. IPL key: $\mathrm{PA}=$ phosphatidic acid, $\mathrm{DH}=$ dihexose, $\mathrm{PE}=$ phosphatidylethanolamine, $\mathrm{PG}=$ phosphatidylglycerol, $\mathrm{MMPE}=$ monomethylphosphatidylethanolamine, $\mathrm{PI}=$ phosphatidylinositol, LPC = lyso-phosphatidylcholine.

\subsubsection{Methane zone slurries}

PC was identified only in three butyrate-amended enrichment slurries, having high abundance in two of them (293717847 peak area/g dry weight on average), which contained sulfate (Fig. 1, 2, 3, Table S1). Trimethylornithine lipid (TMO) was identified in three butyrate slurries in which PC was identified, but at moderate to low abundances (11454294 peak area/g dry weight on average), and was not detected in any of the propionate-amended slurries (Fig. 3). LPC was detected in most of the butyrate- and propionate-amended slurries. The highest LPC abundances were detected in two sulfate containing slurries (23535905 peak area/g dry weight) in which PC and TMO reached to the highest abundance. PE was identified in most of the slurries with higher average abundances in the propionate slurries. DH was identified at moderate to low abundances (between 485170-21381687) in three propionate- and five butyrate-amended slurries incubated at different temperatures with or without sulfate. PG was detected in one propionate- and four butyrate-amended slurries, all five 
of which contained sulfate. PI was identified at relatively low abundances (between 101628-801746) in two propionate- and two butyrate-amended slurries, all incubated at $10^{\circ} \mathrm{C}$ with and without sulfate. MH, betaine, and dimethylphosphatidylethanolamine (DMPE) were all detected at relatively low abundances $(737203,11195317,2801118$ peak area/g dry weight, respectively) in one methane zone slurry that was amended with butyrate and incubated at $25^{\circ} \mathrm{C}$ without sulfate.
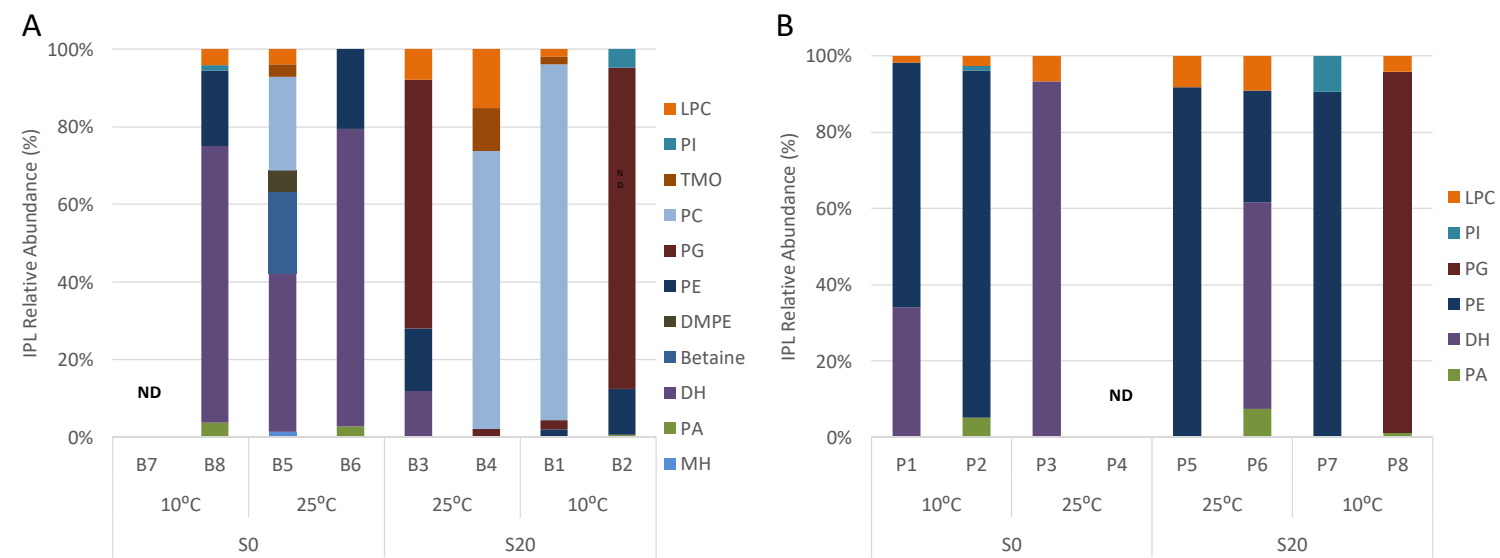

Figure 3: Relative abundance of intact polar lipids (IPLs) in all methane zone enrichment slurries amended with butyrate (A) and propionate (B). IPL relative abundances for cultures B2, B3, B5, B8 from Fig. 1 and $\mathrm{P}_{1}, \mathrm{P}_{3}, \mathrm{P} 6$, P8 from Fig.2 are combined to show full comparison between all methane zone slurries. IPL key: Mhex $=$ monohexose, $\mathrm{PA}=$ phosphatidic acid, $\mathrm{DH}=$ dihexose, $\mathrm{DMPE}=$ dimethylphosphatidylethanolamine, PE = phosphatidylethanolamine, PG = phosphatidylglycerol, $\mathrm{PC}=$ phosphatidylcholine, $\mathrm{TMO}=$ trimethylornithine, $\mathrm{PI}=$ phosphatidylinositol, $\mathrm{LPC}=$ lysophosphatidylcholine. ND: not determined.

\subsubsection{Multivariate analysis}

Canonical correspondence analysis components 1 and 2 explained 30\% and 23\% of variation, respectively, for Bacteria in butyrate-amended slurries; $37 \%$ and $28 \%$ of variation, respectively, for Archaea in butyrate-amended slurries; $42 \%$ and $21 \%$ of variation, respectively, for Bacteria in propionate-amended slurries; and 53\% and 22\% for Archaea in propionate-amended slurries.

In butyrate-amended slurries, the observed bacteria and IPLs clustered into three groups (Fig. 4A). These groups are as followed: [1] PE, PA, OL, PG, and PI clustered more closely with Flavobacteriaceae (BBF), Cytophagaceae (BBC1), Bacteriodetes-SB-5 (BBS5), Marinilabiaceae (BBM), Actinobacteria-OPB41 (BAO), Bacteroidetes-BD2-2 (BBB), Bacteroidetes-VC2.1_Bac22 (BBV), and high sulfate conditions; [2] MH and GlcA clustered more closely with Anaerolineaceae (BCA2), Candidate division OP9 (BCO), Other Bacteria (BO), and low sulfate conditions; [3] PC, LPC, DH, TMO, Betaine, and DMPE clustered more closely with Bacteroidetes-SB-1 (BBS), BacteroidetesWCHB1-69 (BBW), Bacteroidetes-vadinHAı (BBVH), Chloroflexi-GIF9 (BCG), and low sulfate 
conditions. Other than Actinobacteria-OPB41, the rest of the taxa in cluster 1 belonged to Bacteroidetes. Cluster 3 also contained almost exclusively Bacteroidetes with one Actinobacteria taxon, whereas cluster 2 contained three diverse taxa. The observed archaeal taxa in butyrateamended slurries were Methanomicrobia-ANME-1b (EMA), Methanosarcinaceae (EMMM3), Thermoplasmatales-AMOS1A-4113-Do4 (ETTA1), Thermoplasmatales-MBG-D and DHVEG-1 (ETTM), Halobacteriales (EHH), and Miscellaneous Crenarchaeotic Group (TMC). These archaea clustered with group 1 bacteria and IPLs; Methanosaetaceae (EMMM1) clustered with group 2; Methanomicrobiales-(EMM), Methanomicrobiales-C19A (EMMC), Methanomicrobiales-EJ-Eo1 (EMME) clustered with group 3 (Fig. 4B).

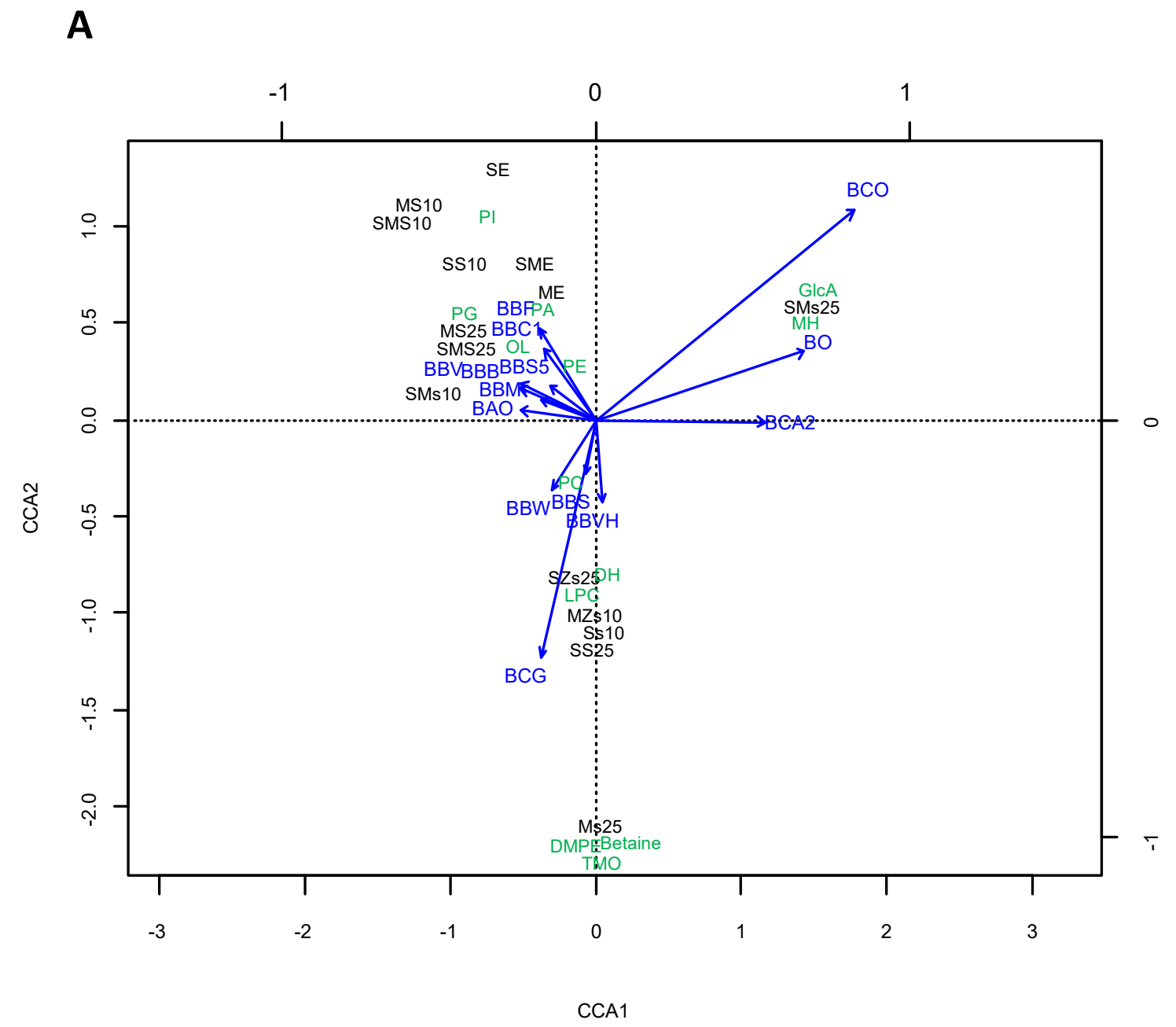




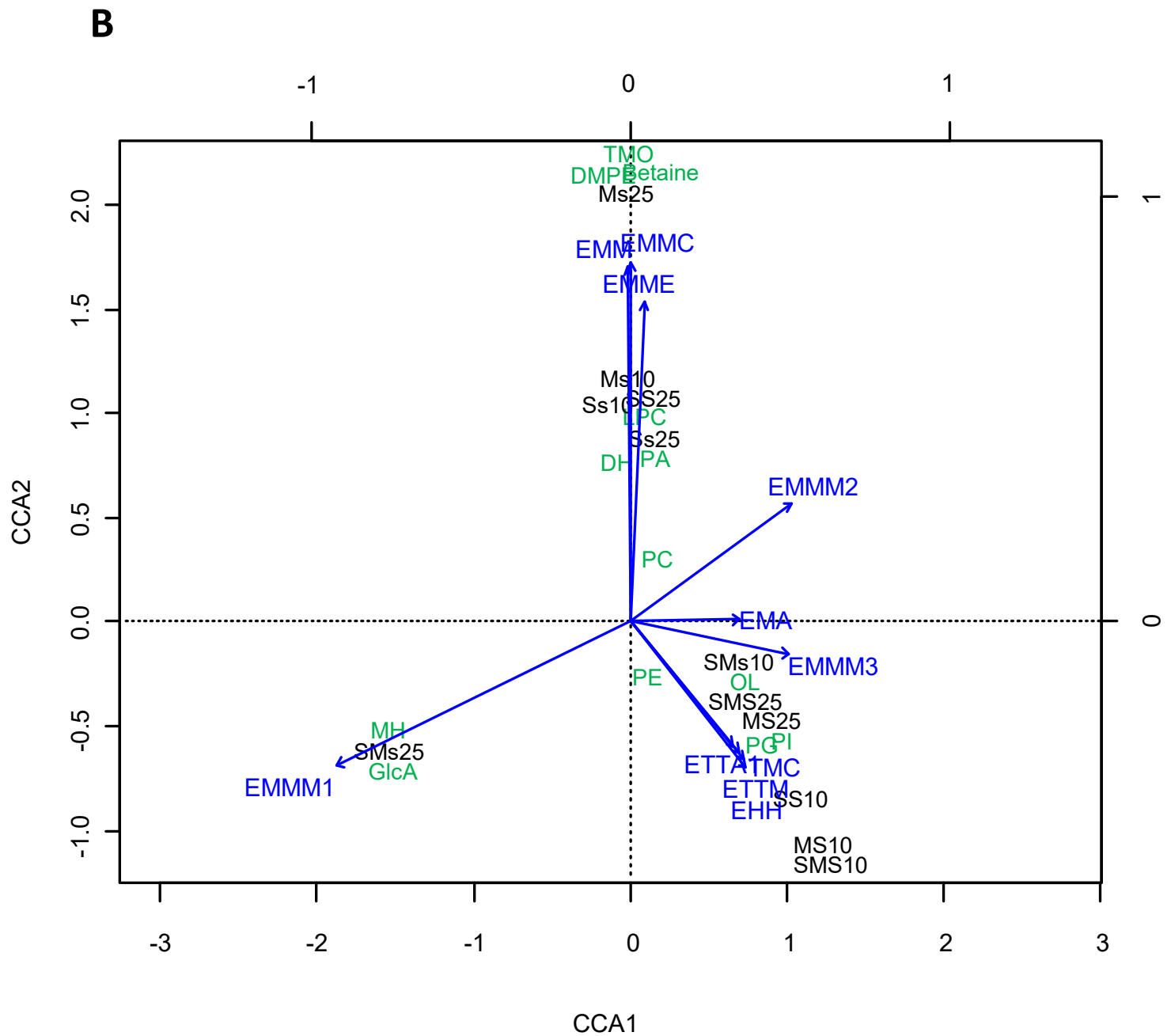

Figure 4: (A) Canonical correspondence analysis of intact polar lipid (IPL) distribution, bacterial taxa distribution, and growth conditions for butyrate-amended enrichment slurries. (B) Canonical correspondence analysis of intact polar lipid (IPL) distribution, archaeal taxa distribution, and growth conditions for butyrate-amended enrichment slurries.

Black text, culture condition key: $\mathrm{S}=$ sulfate zone, $\mathrm{SM}=$ sulfate-methane transition zone, $\mathrm{M}=$ methane zone, $\mathrm{S}=$ high sulfate amendment, $\mathrm{s}=$ low sulfate amendment, $25=25^{\circ} \mathrm{C}, 10=10^{\circ} \mathrm{C}$.

Green text, IPL key: $\mathrm{MH}=$ monohexose, $\mathrm{PA}=$ phosphatidic acid, $\mathrm{DH}=$ dihexose, $\mathrm{DMPE}=$ dimethylphosphatidylethanolamine, $\mathrm{PE}=$ phosphatidylethanolamine, $\mathrm{PG}=$ phosphatidylglycerol, $\mathrm{OL}=$ ornithine, GlcA = glucuronic acid, $\mathrm{PC}=$ phosphatidylcholine, $\mathrm{TMO}=$ trimethylornithine, MMPE = monomethylphosphatidylethanolamine, PI = phosphatidylinositol, $\mathrm{LPC}=$ lysophosphatidylcholine.

Blue text, bacterial taxa key: $\mathrm{BAO}=$ Bacteria - Actinobacteria $-\mathrm{OPB} 41 ; \mathrm{BBB}=$ Bacteria - Bacteroidetes - BD2-2; $\mathrm{BBC}_{1}$ = Bacteria - Bacteroidetes - Cytophagaceae; BBF = Bacteria - Bacteroidetes Flavobacteriaceae; $\mathrm{BBM}=$ Bacteria - Bacteroidetes - Marinilabiaceae $; \mathrm{BBS}=$ Bacteria - Bacteroidetes - SB-1; BBS5 = Bacteria - Bacteroidetes - SB-5; BBV = Bacteria - Bacteroidetes - VC2.1_Bac22; BBVH = Bacteria - Bacteroidetes - vadinHA17; BBW = Bacteria - Bacteroidetes - WCHB1-69; BCA2 = Bacteria - Chloroflexi - Anaerolineaceae; BCG = Bacteria - Chloroflexi - GIF9; BCO = Bacteria - Candidate division $\mathrm{OP}_{9} ; \mathrm{BO}=$ Bacteria other. 
Blue text, archaeal taxa key: EHH = Euryarchaeota - Halobacteria - Halobacteriales; EMA = Euryarchaeota - Methanomicrobia - ANME-1b; EMM = Euryarchaeota - Methanomicrobia Methanomicrobiales; EMMC = Euryarchaeota - Methanomicrobia - Methanomicrobiales - C19A; EMME = Euryarchaeota - Methanomicrobia - Methanomicrobiales - EJ-Eo1; EMMM1 = Euryarchaeota - Methanomicrobia - Methanosarcinales - Methanosaetaceae; EMMM2 = Euryarchaeota - Methanomicrobia - Methanomicrobiales - Methanomicrobiaceae; $\mathrm{EMMM}_{3}=$ Euryarchaeota - Methanomicrobia - Methanosarcinales - Methanosarcinaceae; ETTA1 = Euryarchaeota - Thermoplasmata - Thermoplasmatales - AMOS1A-4113-Do4; ETTM = Euryarchaeota - Thermoplasmata - Thermoplasmatales - Marine Benthic Group D and DHVEG-1; TMC = Thaumarchaeota - Miscellaneous Crenarchaeotic Group.

In propionate-amended slurries, bacterial taxa and IPLs were spread out among more groups as compared to the butyrate cultures. PG and MMPE group were positioned closely to Bacteroidetes-BD2-2 (BBB), Marinilabiaceae (BBM), and $10^{\circ} \mathrm{C}$ culture conditions; PI was positioned closely to Chloroflexi-MSBL5 (BCM); PA was positioned close to Bacteroidetes (BB) and Actinobacteria-OPB41 (BAO); DH was close to Bacteroidetes-SB-1 (BBS); PE and LPC were not positioned near any particular taxon, but clustered closely to low sulfate culture conditions (Fig. 5A). Among archaeal taxa in propionate-amended slurries, PI clustered with Methanomicrobiaceae (EMMM2) and Thermoplasmatales-TMEG; PA with Thermoplasmatales-CCA47 (ETTC); LPC with Methanomicrobiales (EMM); PE with Methanomicrobiales-EJ-Eo1 (EMME) and low sulfate culture conditions (Fig. 5B). 


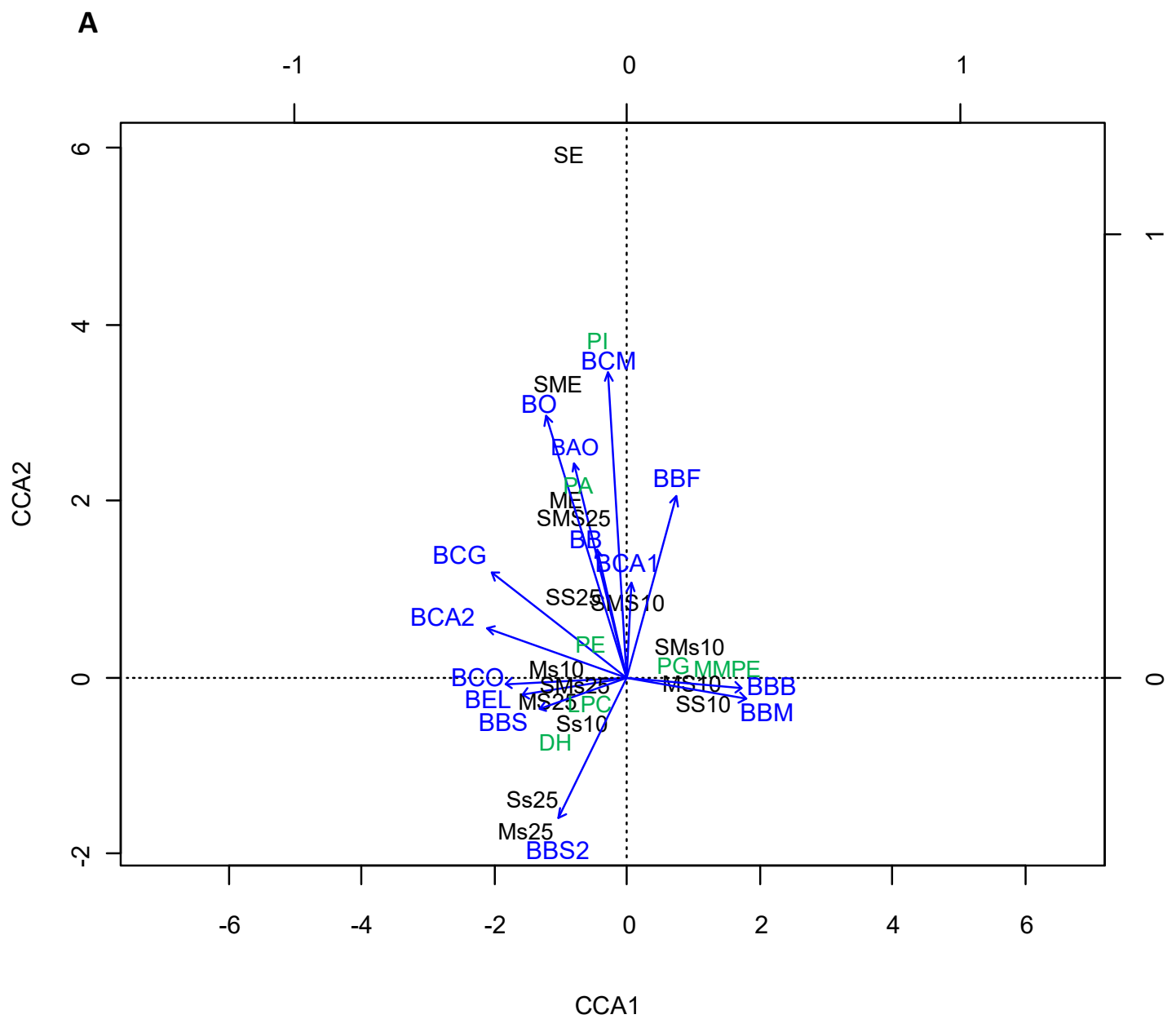




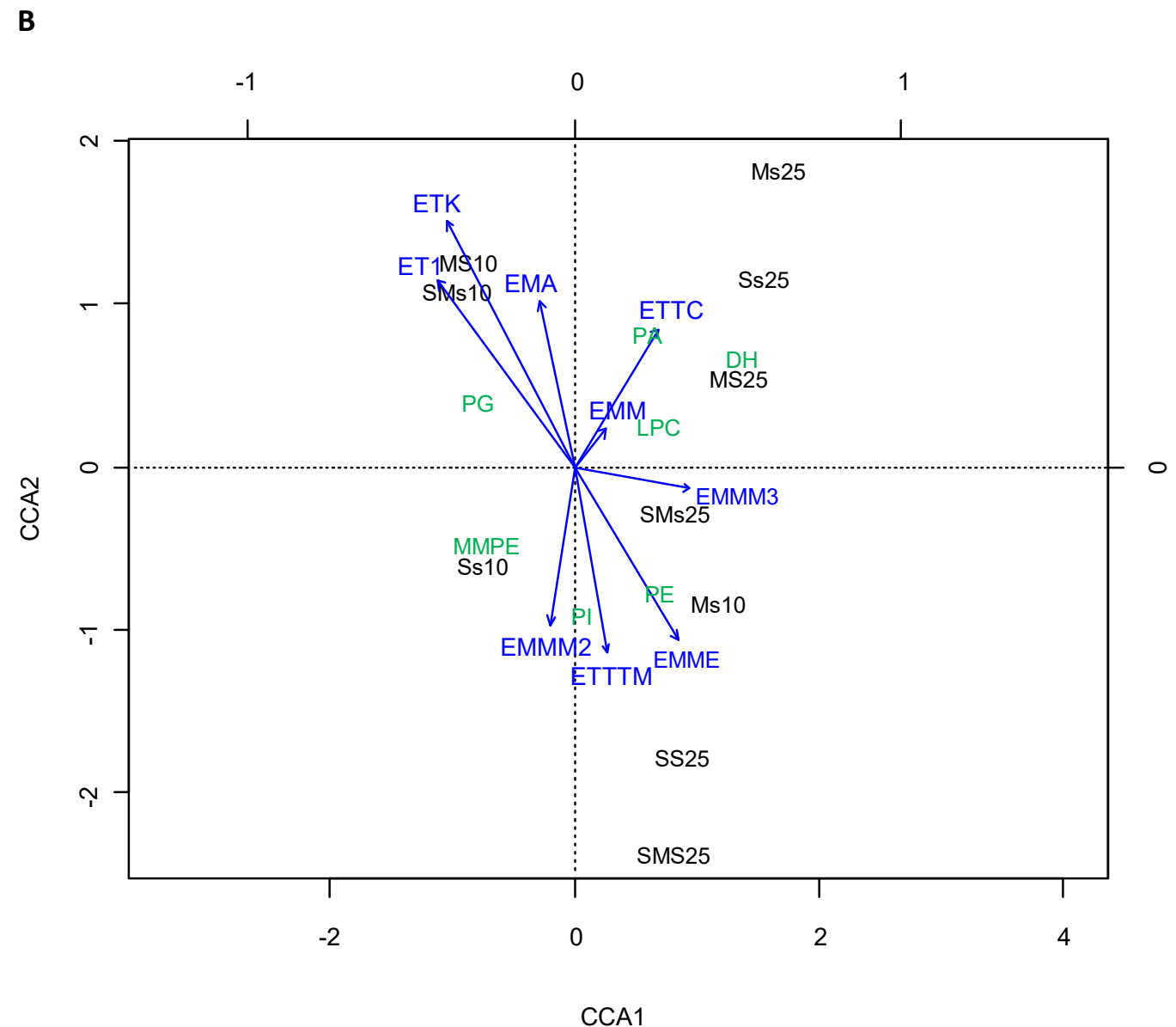

Figure 5: (A) Canonical correspondence analysis of intact polar lipid (IPL) distribution, bacterial taxa distribution, and growth conditions for propionate-amended enrichment slurries. Canonical correspondence analysis of intact polar lipid (IPL) distribution, archaeal taxa distribution, and growth conditions for propionate-amended enrichment slurries.

Black text, culture condition key: $\mathrm{S}$ = sulfate zone, $\mathrm{SM}$ = sulfate-methane transition zone, $\mathrm{M}=$ methane zone, $\mathrm{S}=$ high amended sulfur, $\mathrm{s}=$ low amended sulfur, $25=25^{\circ} \mathrm{C}, 10=10^{\circ} \mathrm{C}$.

Green text, IPL key: PI = phosphatidylinositol, $\mathrm{PA}=$ phosphatidic acid, $\mathrm{PE}=$ phosphatidylethanolamine, $\quad \mathrm{PG}=$ phosphatidylglycerol, $\mathrm{MMPE}=$ monomethylphosphatidylethanolamine, $\mathrm{DH}=$ dihexose, $\mathrm{LPC}=$ lyso-phosphatidylcholine.

Blue text, bacterial taxa key: $\mathrm{BAO}=$ Bacteria - Actinobacteria $-\mathrm{OPB} 41$; $\mathrm{BB}=$ Bacteria - Bacteroidetes; $\mathrm{BBB}=$ Bacteria - Bacteroidetes $-\mathrm{BD} 2-2 ; \mathrm{BBF}=$ Bacteria - Bacteroidetes - Flavobacteriaceae $; \mathrm{BBM}=$ Bacteria - Bacteroidetes - Marinilabiaceae; BBS = Bacteria - Bacteroidetes - SB-1; BBS2 = Bacteria Bacteroidetes - Sphingobacteriales; $\mathrm{BCA1}=$ Bacteria - Chloroflexi - Anaerolineae; $\mathrm{BCA2}=$ Bacteria Chloroflexi - Anaerolineaceae; BCG = Bacteria - Chloroflexi - GIF9; BCM = Bacteria - Chloroflexi MSBL5; $\mathrm{BCO}=$ Bacteria - Candidate division OP9; BEL = Bacteria - Elusimicrobia - Lineage_IV; BO $=$ Bacteria other.

Blue text, archaeal taxa key: EMA = Euryarchaeota - Methanomicrobia - ANME-1b; EMM = Euryarchaeota - Methanomicrobia - Methanomicrobiales; EMME = Euryarchaeota Methanomicrobia - Methanomicrobiales - EJ-Eo1; EMMM2 = Euryarchaeota - Methanomicrobia Methanomicrobiales - Methanomicrobiaceae; EMMM3 = Euryarchaeota - Methanomicrobia - 
Methanosarcinales - Methanosarcinaceae; ETK = Euryarchaeota - Thermoplasmata - Kazan-3A-21; ET1 = Euryarchaeota - Thermoplasmata - 19c-33; ETTC = Euryarchaeota - Thermoplasmata Thermoplasmatales - CCA47; ETTTM = Euryarchaeota - Thermoplasmata - Thermoplasmatales Terrestrial Miscellaneous Gp (TMEG).

\subsection{Discussion}

The greater diversity of microbial taxa versus IPLs agrees with consistent findings that many IPLs with the same head group and/or core lipid structures are produced by a wide range of microbial species (Fang and Barcelona, 1998; Fang et al., 2000; Schouten et al., 200o; Rütters et al. 2002a; 2002b; Sturt et al., 2004; Lipp et al., 2008; Schubotz et al., 2009). The higher concentrations of bacterial IPLs in samples are generally consistent with higher concentrations of sedimentary organic carbon which likely serves as major substrate (Lipp et al., 2008). Both the microbial community and IPL distribution changed much from the sediment samples compared to the sediment slurries inoculated with corresponding sediment samples, indicating that incubation conditions (temperature, sulfate concentration, carbon source) have an impact on the cell membrane composition of different microbial communities. Twice as many IPL head group structures were observed in butyrate-amended sediment slurries than propionate-amended sediment slurries showing that the butyrate degrading community has more diverse membrane composition.

The most abundant IPLs identified in most of the sediment enrichment slurries are phosphatidylethanolamine (PE), which are often find in aquatic environments and sediments and are attributed to sulfate-reducing bacteria (Rossel et al., 2008). The high abundance of SRB in enrichment slurries (Chapters 3 and 4 ) and the positive correlation between PE and high sulfate condition (Fig $4 \mathrm{~A}$ ) indicates that the occurrence of PE can be linked to the presence of SRB in enrichment slurries. Other IPLs that showed high relative abundance among sediment slurries were PG and PC. PG has been identified as the second most abundant lipid in bacterial membranes (Dowhan, 1997) including cultured representatives of the deep biosphere (Schubotz, 2005). PC has been identified in more than $10 \%$ of all bacteria (Sohlenkamp et al., 2003). The higher relative abundance of PC in only butyrate-amended enrichment slurries therefore suggests a specific bacterial group involving in butyrate conversion might posses PC.

The identification of TMO lipids in three butyrate-amended methane zone enrichment slurries (Fig. 3, Table S1) was surprising since these lipids were originally identified in planctomycete isolates from ombrotrophic northern wetlands (Moore et al., 2013), and peaked in 
abundance at the oxic-anoxic interface (Moore et al., 2015b). These ombrotrophic wetlands are methanogenic, suggesting TMOs could be linked to the methanogenic microbial communities in different environments. The TMOs identified in the butyrate-amended methane zone sediment slurries contained core lipids $\mathrm{C}_{18}: \mathrm{o} / \beta \mathrm{OH}-\mathrm{C}_{18} 8: 0, \mathrm{C}_{18}: 1 / \beta \mathrm{OH}-\mathrm{C}_{18}: \mathrm{o}, \mathrm{C}_{16}: \mathrm{o} / \beta \mathrm{OH}-\mathrm{C}_{18}: 0$, and $\mathrm{C}_{19}: 1 / \beta O H-C_{18}$ :o. TMOs with core lipids $\mathrm{C}_{18}: 1 / \beta \mathrm{OH}-\mathrm{C}_{18}: 0, \mathrm{C}_{16}$ :o/ $\beta \mathrm{OH}-\mathrm{C}_{18}$ :o, and $\mathrm{C}_{19}: 1 / \beta \mathrm{OH}-\mathrm{C}_{18}: 0$ were also identified in northern wetland planctomycete isolates (Moore et al., 2013), and TMOs with core lipids $\mathrm{C}_{18}$ :1/ $\beta \mathrm{OH}-\mathrm{C}_{18}$ :o and $\mathrm{C}_{16}$ :o/ $\beta \mathrm{OH}-\mathrm{C}_{1} 8$ :o were also identified in northern wetland peats (Moore et al., 2015b). No planctomycete phylotypes were identified using pyrosequencing analysis in any of the butyrate-amended methane zone sediment slurries indicating that the observed TMOs were produced by other microbial groups or the relative abundance of the responsible organisms very low. TMOs have also recently been identified in meso-oligotrophic lakes of Minnesota and Iowa, possibly as a response to low phosphorus concentrations (Bale et al., 2016).

Multivariate analysis showed that the Bacteroidetes phylum, and to a lesser extent, Methanomicrobiales order contributed to the variation in IPL distribution compared to other abundant phylogenetic groups in butyrate-amended slurries. In propionate-amended slurries, Bacteriodetes and Methanomicrobiales had also greater contributions than various other microbial groups. The sulfate concentrations in butyrate-amended sediment slurries and $10^{\circ} \mathrm{C}$ as incubation temperature in the propionate-amended slurries contributed to the microbial composition and IPL variation (Fig. 4 and 5). Various Bacteriodetes species have been observed to produce a wide range of IPLs including PEs, ornithine lipids, and lysine lipids (LLs) (Moore et al., 2015a; 2016; Singh et al., 2015). Recently, many marine and estuarine Flavobacteriaceae related species, were found to produce PEs and amino lipids as their most abundant membrane lipid structures (Yoon and Kasai, 2016; Jung et al., 2016; Liu et al., 2016; Wang et al., 2016; Park et al., 2016; Liu et al., 2016; Song et al., 2015). The Flavobacteriaceae family belong to the Bacteriodetes phylum and is clustered closely with PEs and OLs (Fig. 4A). This indicates that Flavobacteriaceae contributed to the variation in PEs and OLs in cluster 1 (Fig. 4A). Marine sediment and seawater members of Halobacteriales have been reported to produce PGs and PEs (Wang et al., 2010a; Yim et al., 2014) indicating that these taxa contributed to the variation of these IPLs in cluster 1.

This study shows that the membrane structures of microbes inhabiting sulfate, sulfatemethane transition and methane zones of marine sediments can be highly variable among different taxa and depending on different incubation conditions, such as temperature, substrate, and 
electron acceptor availability. The presence of trimethylornithine lipids (TMOs) in methane zone slurries indicates that these IPLs can be found in a wider range of ecosystems than previously observed. Further study is needed to shed light on how microbial communities in coastal marine sediment respond to changes in environmental and incubation conditions. Such studies will be extremely important given the high level of human impact on coastal ecosystems (Syvitski et al., 2005; Talaue-McManus L. 2010; Deegan et al., 2012; Halpern et al., 2015). 


\section{Supplementary data}

Table S1. Relative abundance (HPLC/MS base peak area/g sediment dw [dry weight]) of all IPLs identified in Aarhus Bay sediment and the enrichment slurries inoculated with this sediment. 120 $\mathrm{cm}=$ sulfate zone, $135-165 \mathrm{~cm}=$ sulfate-methane transition zone, $180-300 \mathrm{~cm}=$ methane zone.

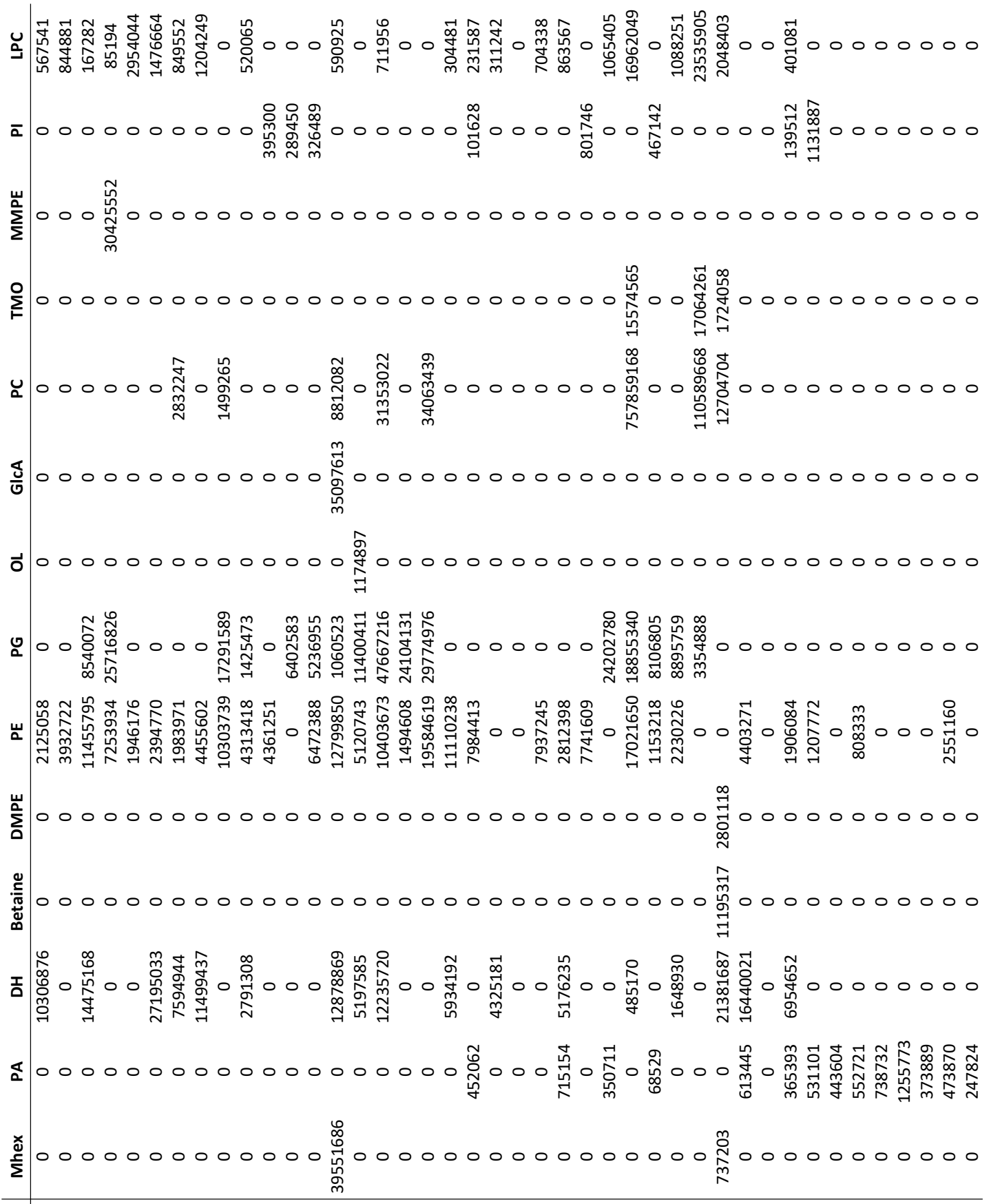

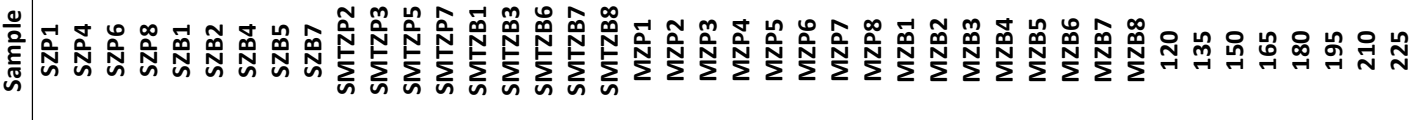




\section{Chapter 6}

\section{Physiological and molecular characterization of anaerobic marine propionate- and butyrate-converting syntrophic cultures}

Derya Ozuolmez, M. Cristina Gagliano, Daan van Vliet, Alfons J.M. Stams, Caroline M. Plugge 


\section{Abstract}

Degradation of propionate and butyrate in marine sediments is carried out predominantly by sulfate-reducing bacteria. However in sulfate-depleted zones, these compounds are degraded by methanogenic communities, which have been poorly studied. Here, we studied anaerobic conversion of propionate and butyrate under sulfate-free and low-sulfate conditions by syntrophic communities enriched from different biogeochemical zones of Aarhus Bay. Several transfers from previously enriched sediment slurries were performed. Butyrate conversion after four transfers occurred rapidly and acetate and methane and/or sulfide accumulated in the cultures. On the other hand, propionate conversion proceeded slow enabling enrichment of acetoclastic methanogens and hence the complete conversion of propionate. The addition of low amounts of sulfate to the cultures did not inhibit syntrophic conversion, instead, both sulfate reduction and methanogenesis proceeded concomitantly. The butyrate-converting cultures were dominated by Syntrophomonas bryantii and an uncultured Syntrophomonas species. The propionate-converting cultures were dominated by bacteria that are phylogenetically similar to Cryptanaerobacter phenolicus and different Pelotomaculum species (95-96\%, 16S rRNA gene based), indicating presence of possible novel species of propionate-converting syntrophs. Low sulfate amended butyrate- and propionateconverting cultures contained Desulfobacteraceae members together with uncultured Syntrophomonas sp. and Cryptanaerobacter sp., respectively. Hydrogenotrophic methanogens were present in all enrichment cultures, whereas aceticlastic methanogens were abundant in sulfate-free cultures but had low abundance in sulfate-amended cultures. 


\subsection{Introduction}

Propionate and butyrate are important intermediates in anaerobic degradation of organic matter in marine sediments. These compounds can be oxidized by a variety of marine sulfate-reducing bacteria (SRB) either completely to $\mathrm{CO}_{2}$ or incompletely to acetate (Widdel, 1988; Muyzer and Stams, 2008). Bacteria that can couple propionate and butyrate oxidation to sulfate reduction include members of the families Desulfobacteraceae, Desulfobulbaceae, Syntrophobacteraceae, Peptococcaceae (Kuever et al., 2014b; Stackebrandt, 2014). In sulfate-depleted sediments, organic matter is degraded through syntrophic interactions of acetogenic bacteria and methanogens. In sulfate-depleted sediments, methanogens consume hydrogen, formate and acetate that are formed as products of organic carbon degradation. Hydrogen and formate consumption allows propionate and butyrate conversion to be energetically favorable (McInerney et al., 1979; Boone and Bryant, 1980). In this way, a syntrophic relationship is established between bacteria and methanogens (Schink and Stams, 2013; McInerney et al., 2008).

Syntrophic propionate-oxidizing bacteria are affiliated with two phylogenetic groups of bacteria. The first group contains Gram-negative genera Syntrophobacter and Smithella, belonging to the Deltaproteobacteria. The second group contains Gram-positive bacteria affiliated to genera Pelotomaculum and Desulfotomaculum, belonging to the phylum Firmicutes (Boone and Bryant, 1980; Harmsen et al., 1998; Wallrabenstein et al., 1995; Imachi et al. 2002; Plugge et al. 2002; de Bok et al. 2005; Chen et al., 2005; Stams et al., 1993). These genera are phylogenetically associated to sulfate-reducing bacteria and some of these syntrophs can couple propionate oxidation to sulfate reduction. On the other hand, there are few species that are obligately syntrophic and hence unable to reduce sulfate; these are Pelotomaculum schinkii (de Bok et al., 2005) and Pelotomaculum propionicicum (Imachi et al., 2007).

Most syntrophic butyrate-degrading bacteria are classified as low GC Gram-positive bacteria. Organisms capable of syntrophic butyrate metabolism include all species of Syntrophomonas (Stieb and Schink, 1985; Zhao et al., 1990; McInerney et al. 1981; Lorowitz et al. 1989; Zhang et al. 2004; Zhang et al. 2005; Roy et al., 1986; Sousa et al. 2007a; Wu et al. 2006a; Wu et al. 2006b; Wu et al. 2007), Thermosyntropha lipolytica (Svetlitshnyi et al. 1996), Syntrophothermus lipocalidus (Sekiguchi et al. 2000) and the Gram-negative Syntrophus aciditrophicus (Jackson et al, 1999). The majority of the Syntromonadaceae family members are not obligate syntrophs as they can dismutate crotonate without a syntrophic partner (McInerney et al., 
1979, 1981; Stieb and Schink, 1985; Beaty and McInerney, 1987; Lorowitz et al., 1989; Zhao et al., 1990, 1993; Svetlitshnyi et al., 1996; Sekiguchi et al., 2000; Zhang et al., 2004; Hatamoto et al., 2007). The exceptions to this are Syntrophomonas sapovorans and S. zehnderi, which degrade all linear saturated fatty acids with 4 to 18 carbon atoms only in coculture with methanogens (Roy et al., 1986; Sousa et al., 2007a). A syntrophic butyrate-degrading bacterium, Algorimarina butyrica, was isolated from marine sediments (Kendall et al., 2006). This bacterium is phylogenetically affiliated to Deltaproteobacteria and closely related to the sulfate-reducing Desulfonema magnum. Yet, it cannot couple butyrate oxidation to sulfate reduction (Kendall et al., 2006). A. butyrica is the first and the only marine syntrophic butyrate degrader isolated.

The main goal of this study was to establish marine propionate- and butyrate-degrading syntrophic consortia in enrichment cultures using the sediment slurry enrichments obtained in chapters 3 and 4. Based on this previous research, we proposed that Cryptanaerobacter/Pelotomaculum phylotype converts propionate and Syntrophomonas and Desulfobacteraceae family members convert butyrate in syntrophy with methanogens in Aarhus Bay sediment. In order to further enrich these microorganisms and to confirm the results of our previous study, the enrichment slurries were sub-cultured for four times. Here, propionate and butyrate degradation and product formation was quantified, and the eventual microbial community composition in the fourth sub-cultures was analyzed.

\subsection{Materials and Methods}

\subsubsection{Source of inocula and preparation of sub-cultures}

Propionate and butyrate degrading sediment slurries were set up using sediment taken from sulfate zone (SZ), sulfate-methane transition zone (SMTZ) and methane zone (MZ) of Station Mi in Aarhus Bay, Denmark. 1 L glass bottles were used to mix the sediment with marine anoxic mineral salt medium (see Chapter 3) containing $10 \mathrm{mM}$ propionate or butyrate as carbon sources with and without $20 \mathrm{mM}$ sulfate in sulfate zone and methane zone slurries, and with $3 \mathrm{mM}$ and $20 \mathrm{mM}$ sulfate for sulfate-methane transition zone slurries. The overview of all butyrate- and propionate-amended sediment slurries is given in chapter 3 and 4 . The slurries were regularly monitored for substrate consumption and product formation and regular additions of propionate, butyrate and/or sulfate were performed. These enrichment slurries have been maintained for 514-571 days. Subsequently, sediment-free transfers were performed from original slurries into culture bottles containing the 
same medium as was used to prepare enrichment slurries. The medium content and preparation was described in chapter 3. After three transfers, the culture bottles were without sediment particles and the fourth transfer was used to quantify propionate and butyrate conversion and to analyze the microbial composition of the enrichment cultures.

Enrichment cultures were inoculated in triplicate with $20 \mathrm{ml}$ of original slurry into $250 \mathrm{ml}$ serum vials with $134 \mathrm{ml}$ of anoxic mineral salts medium (including carbon source, reductant, and vitamin solution). $10 \mathrm{mM}$ butyrate or propionate was used as carbon source with $3 \mathrm{mM}$ sulfate as electron acceptor for SMTZ and without sulfate for SZ and MZ cultures. The enrichment cultures were incubated statically in the dark at $25^{\circ} \mathrm{C}$ with a gas composition of $\mathrm{N}_{2} / \mathrm{CO}_{2}(80: 20 \mathrm{v} / \mathrm{v})$ at 1,7 atm pressure.

\subsubsection{Analytical methods}

Methane in the headspace of the culture bottles was analyzed by gas chromatography with a Shimadzu GC-14B (Shimadzu, Kyoto, Japan) equipped with a packed column (Molsieve 13X, 6o-80 mesh, $2 \mathrm{~m}$ length, $3 \mathrm{~mm}$ internal diameter; Varian, Middelburg, The Netherlands) and a thermal conductivity detector set at $70 \mathrm{~mA}$. The oven and the injector temperatures were both $100^{\circ} \mathrm{C}$. The detector temperature was $150^{\circ} \mathrm{C}$. Argon was the carrier gas at a flow rate of $30 \mathrm{ml} \mathrm{min}^{-1}$.

Propionate, butyrate and acetate were quantified using a Thermo Scientific Spectrasystem HPLC system (Thermo Scientific, Waltham, MA) equipped with a Varian Metacarb 67H $300 x 6.5$ mm column (Agilent, Santa Clara, MA) connected to a UV and Refractive Index (RI) detector. 0.005 M sulfuric acid was used as eluent and $10 \mathrm{mM}$ sodium crotonate as internal standard. The flow rate

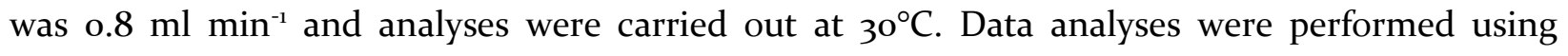
ChromQuest (Thermo Scientific, Waltham, MA) and Chromeleon software (Thermo Scientific, Waltham, MA).

Sulfate and sulfide in SMTZ cultures were measured as described in Chapter 3.

\subsubsection{DNA extraction}

Genomic DNA was extracted from the propionate and butyrate-amended cultures using Fast DNA Kit for Soil (MP Biomedicals, Santa Ana, CA) according to the manufacturer's instructions. Two 45second beat beating steps were applied using a Fastprep Instrument (MP Biomedicals, Santa Ana, 
CA). The DNA quality was examined on $1 \%(\mathrm{w} / \mathrm{v})$ agarose gel and the DNA quantity was determined using Nanodrop 1000 spectrophotometer (Thermo Fisher Scientific, Wilmington, DE).

\subsubsection{Clone library construction}

Extracted DNA was used for a clone library construction. Almost full-length 16S rRNA genes were amplified using primers 27F (5'-AGAGTTTGGATCMTGGCTCAG-3') (Lane, 1991) and 1369R (5'GCCCGGGAACGTATTCACCG-3') (Iwamoto et al., 2000) for Bacteria, and the primers 25F (5'CYGGTTGATCCTGCCRG-3') (Dojka et al., 1998) and 1386R (5'-GCGGTGTGTGCAAGGAGC-3') (Skillmann et al., 2004) for Archaea. PCR amplification was carried out using GoTaq DNA Polymerase kit (Promega, Madison, WI, USA). Bacterial 16S rRNA amplification was carried out as described in Sousa et al. (2007b), while archaeal amplicons were obtained following the protocol of Borrel et al. (2012). 16S rRNA gene amplicons' integrity and length was checked on agarose gel and subsequently purified with Zymoclean Gel DNA Recovery Kit (Zymo Research, Orange, CA, USA). Ligation and cloning of the PCR products were performed with the use of a PGEM ${ }^{\circledR}-\mathrm{T}$ Easy Vector kit (Promega, Madison, WI, USA) and E. coli XL blue competent cells (Agilent Technologies, Santa Clara, USA). White colonies were randomly selected and transferred to a 96 well Masterblock ${ }^{\circledR}$ plate (Greiner Bio-One, the Netherlands). Nearly full-length 16S rRNA genes were sequenced by GATC Biotech (Konstanz, Germany) using the primers $\mathrm{T}_{7}$ and SP6. Sequences were manually trimmed and checked for chimeras using VecScreen (http://www.ncbi.nlm.nih.gov/tools/vecscreen/) and Decipher (http://decipher.cee.wisc.edu/FindChimeras.html). Sequence similarity was checked using NCBI MegaBlast (http://blast.ncbi.nlm.nih.gov/Blast.cgi). Data analysis of amplicons was carried out with the SilvaNGS software pipeline (https://www.arb-silva.de/ngs).

\subsubsection{Fluorescence in situ hybridization (FISH)}

FISH analysis was performed on paraformaldehyde-fixed biomass samples taken from the enrichment cultures, according to the procedure described in Amann et al. (1995). Three samples per each culture condition were analyzed to ensure the complete analysis of the microbial dynamics. Oligonucleotide probes were specific for Bacteria (EUB338mix probes) and Archaea (ARC915 probe) domains. Details of the employed oligonucleotide probes are available at probeBase (Greuter et al., 2016). Probes were labelled with Cу3 or FITC fluorophores. All the hybridizations with specific probes were carried out in combination with DAPI staining, to highlight the probe 
coverage versus the total community. Samples were examined by epifluorescence microscopy (Olympus BX41) equipped with Infinity Camera (Lumenera corporation, Canada). FISH images were further modified using the ImageJ software package (version1.37v, Wayne Rasband, National Institute of Health, Bethesda, MD, USA, available in the public domain at http://rsb. info.nih.gov/ij/index.html).

\subsection{Results and discussion}

\subsubsection{Butyrate conversion}

The conversion of butyrate was studied under sulfate-reducing and methanogenic conditions in enrichment cultures that were derived from three enrichment slurries prepared with SZ, SMTZ and MZ sediment (Figure 1). Butyrate was completely metabolized within 11 days in all enrichment cultures. In the SZ culture, $18.0 \pm 1.37 \mathrm{mM}$ butyrate was converted to $27.3 \pm 0.6 \mathrm{mM}$ acetate and 8.8 $\pm 0.3 \mathrm{mM}$ methane (Table 1, Figure la). Acetate and methane were produced in the MZ culture (Figure 1c). The ratios of consumed butyrate to produced acetate and methane in SZ and MZ cultures are $1: 1.5: 0.5$ and $1: 1.6: 0.4$, respectively. These values are close to the predicted stoichiometric values of syntrophic butyrate degradation (Butyrate $+0.5 \mathrm{HCO}_{3}{ }^{-}+0.5 \mathrm{H}_{2} \mathrm{O} \rightarrow 2$ $\left.\mathrm{CH}_{3} \mathrm{COO}^{-}+0.5 \mathrm{CH}_{4}+0.5 \mathrm{H}^{+}\right)($Chapter 3-Table 1, reaction 7).

In low-sulfate amended SMTZ cultures, butyrate conversion was coupled to both sulfate reduction and methanogenesis (Fig lb, le). $15.8 \pm 0.4 \mathrm{mM}$ butyrate was degraded and $4.2 \pm 0.5 \mathrm{mM}$ sulfate was reduced. Here, $27.3 \pm 0.9 \mathrm{mM}$ acetate, $5.1 \pm 0.1 \mathrm{mM}$ sulfide and $5.9 \pm 0.5 \mathrm{mM}$ methane were produced (Table 1). Two possible routes for butyrate conversion in these enrichments could have occurred: (1) Butyrate oxidation via sulfate reduction (Chapter 3-Table 1, reaction 2) plus concurrent syntrophic butyrate conversion with acetate- and hydrogen-dependent sulfate reduction and/or methanogenesis (Chapter 3-Table 1, reactions $1+3,4,5$ and/or 6), (2) Syntrophic butyrate conversion with acetate- and hydrogen-dependent sulfate reduction and/or methanogenesis (Chapter 3-Table 1 , reactions $1+3,4,5$ and/or 6). In case of the first route, both a sulfate reducer and an acetogen convert butyrate. 
Table 1. The overview of substrate consumption and product formation in the enrichment cultures. The values represented in the table are the total amounts of the reactants consumed and the products formed along the study. All cultures were prepared in triplicate. The values are the mean of triplicates for butyrate SMTZ and MZ cultures, duplicates for butyrate SZ and propionate SMTZ and MZ. The two replicates of propionate SZ culture did grow slower, thus the values from only one replicate of this culture is presented here. NA: not applicable, NM: not measured. Hydrogen or formate were not detected in any of the incubations.

\begin{tabular}{|c|c|c|c|c|c|c|c|c|}
\hline \multirow{2}{*}{ Substrate } & \multirow{2}{*}{ Origin } & \multirow{2}{*}{ Time (d) } & \multicolumn{3}{|c|}{ Reactants (mM) } & \multicolumn{3}{|c|}{ Products (mM) } \\
\hline & & & Butyrate & Propionate & Sulfate & Acetate & Methane & Sulfide \\
\hline \multirow{3}{*}{ Butyrate } & SZ & 11 & $18.0 \pm 1.4$ & NA & NA & $27.3 \pm 0.6$ & $8.8 \pm 0.3$ & NM \\
\hline & SMTZ & 11 & $15.8 \pm 0.4$ & NA & $4.2 \pm 0.5$ & $27.3 \pm 0.9$ & $5.9 \pm 0.5$ & $5.1 \pm 0.1$ \\
\hline & $M Z$ & 11 & $15.8 \pm 0.4$ & NA & NA & $25.8 \pm 0.5$ & $6.2 \pm 0.3$ & NM \\
\hline \multirow{3}{*}{ Propionate } & SZ & 70 & NA & 18.2 & NA & 0.78 & 24.5 & NM \\
\hline & SMTZ & 297 & NA & $17.7 \pm 0.7$ & NM & $15.1 \pm 1.0$ & $10.3 \pm 0.4$ & NM \\
\hline & $M Z$ & 297 & NA & $17.9 \pm 0.0$ & NA & $1.5 \pm 0.0$ & $26.4 \pm 1.6$ & NM \\
\hline
\end{tabular}

In the second, butyrate is converted only by an acetogen to acetate and hydrogen and hydrogen is consumed by a methanogen and part of the acetate is oxidized by a sulfate reducer (Fig le).

Previous studies have reported the existence of syntrophic butyrate degradation in sulfate containing ecosystems, such as marine sediments, anaerobic digesters and batch cultures (Kendall et al., 2006; Visser et al., 1993; Struchtemeyer et al., 2011). The results of our study support previous findings and show that under low sulfate concentrations syntrophic butyrate degradation can occur simultaneously with sulfate reduction (Fig lb, le). Methanogenesis can be the dominant pathway for the utilization of the products $\mathrm{H}_{2}$ and/or formate, whereas acetate is probably utilized via sulfate reduction (Fig le, Table 1). This is supported by the absence of aceticlastic methanogens in SMTZ culture as revealed by community analysis (Fig 2e, Table S2). 

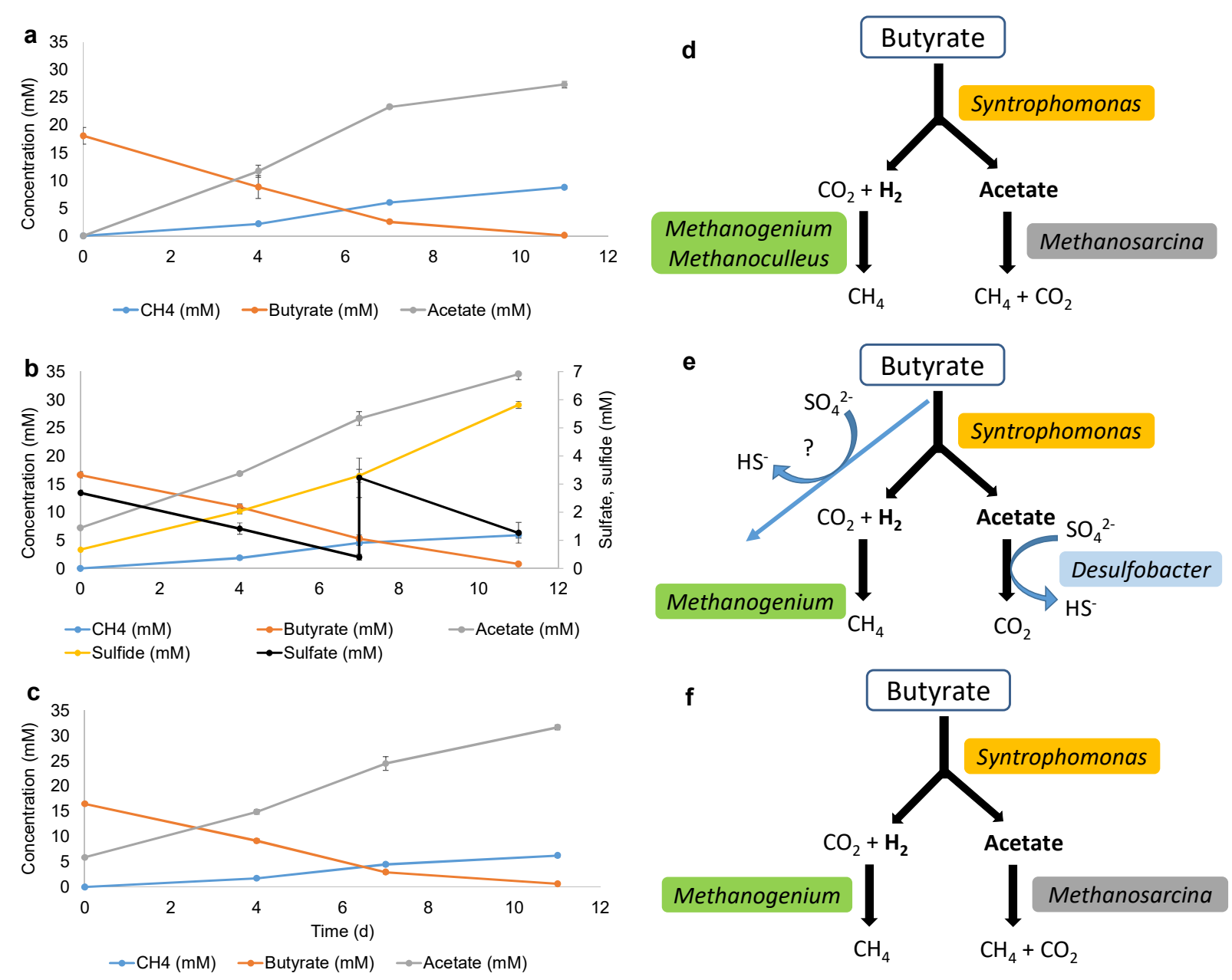

Figure 1. Butyrate conversions (SZ (a), SMTZ (b) and MZ (c)) and proposed butyrate conversion routes (SZ (d), SMTZ (e) and MZ (f)) in the cultures. Butyrate conversion routes are proposed based on microorganisms with high relative abundance and observed butyrate conversion stoichiometries. Values are mean of biological duplicates for SZ and triplicates for SMTZ and MZ. Error bars indicate the standard deviations.

\subsubsection{Microbial communities in butyrate enrichments}

Bacterial 16S rRNA based clone libraries of butyrate-degrading enrichment cultures after 11 days of incubation revealed dominance of Syntrophomonas (Figure 2a, 2b, 2c and Table S1). Within the clone library, 16 clones derived from the SZ culture (53\%) and 23 clones derived from the MZ culture (77\%) showed 99\% similarity to Syntrophomonas bryantii (Figures 2a and 2c). S. bryantii is a Grampositive, spore-forming, butyrate degrading syntroph isolated from marine mud (Stieb and Schink, 1985). The ability of $S$. bryantii to form spores can be an advantage to survive under unfavorable conditions in marine sediments. This ability might explain the dominance of S. bryantii, a marine 
isolate, in both sulfate and methane zone enrichment cultures. $42 \%$ of the clones derived from the SMTZ culture affiliated with an uncultured Syntrophomonas sp. (95\% similarity) (Figure 2b and Table S1). Low sulfate concentration in SMTZ culture might have stimulated Syntrophomonas to reach as high relative abundance as in the SZ and MZ cultures. Sulfate-reducing bacteria that directly couple butyrate oxidation to sulfate reduction grow faster than syntrophic butyrate degraders. On the other hand, the growth rates of some syntrophic butyrate degraders were reported to be higher than some butyrate-oxidizing sulfate-reducers (Oude Elferink et al., 1994). This might be due to their similar kinetic properties rather than thermodynamic factors since Gibbs free energy changes for the complete oxidation of butyrate coupled to sulfate reduction $\left(\Delta \mathrm{G}^{\circ}\right.$ of $6.1 \mathrm{~kJ}$ per electron transferred) or to methane production $\left(\Delta \mathrm{G}^{\circ}\right.$ of $-4.1 \mathrm{~kJ}$ per electron transferred) are in the same range (Chapter 3, Table 1) (McInerney and Beaty, 1988). These reasons may allow syntrophic butyrate degraders to exist in sulfate-reducing ecosystems such as marine sediments and aquifers (Kendall et al., 2006; Struchtemeyer et al., 2011). Similarly, Syntrophomonas in our study could take part in butyrate degradation in the presence of sulfate, with both sulfate reducers and methanogens as partner organisms (Fig le). The second most abundant phylotype (26\%) in the SMTZ culture was affiliated to Deltaproteobacteria (99\%) and had 98\% similarity to Desulfobacter latus (Figure 2b and Table Sl). A D. latus related bacterium was the dominant acetate-degrading organism after several sub-culturing period in low-sulfate amended SMTZ culture (Kuever et al., 2014b) and probably was the syntrophic partner of Syntrophomonas. Despite the higher free-energy change associated with acetate oxidation by sulfate $\left(\Delta \mathrm{G}^{\circ}\right.$ of $\left.-47.6 \mathrm{~kJ} / \mathrm{mol}\right)$ as compared to acetate cleavage to $\mathrm{CH}_{4}$ and $\mathrm{CO}_{2}\left(\Delta \mathrm{G}^{\circ}\right.$ of $\left.-31 \mathrm{~kJ} / \mathrm{mol}\right)$, Schönheit et al. (1982) has explained that the difference in substrate affinities can account for the inhibition of methanogenesis from acetate in sulfate-rich environments. In Chapter 2, we report minor competition between acetate-consuming D. latus and Methanosaeta concilii for acetate in a mixed coculture. Butyrate in the SMTZ culture was converted solely by Syntrophomonas and $\sim 4 \mathrm{mM}$ acetate was consumed (Table 1) by D. latus while hydrogen was utilized by Methanogenium cariaci, a marine methanogen (Romesser et al., 1979) to produce methane (Fig le, 2e). This observation was further supported by FISH analysis on SMTZ culture fixed after 16 days of cultivation, highlighting the presence of one morphotype positive to the ARC915 probe (Fig S2), which is in agreement with the clone library results (Fig 2e). Two morphologies were detected with the bacterial EUB338 probe. These morphotypes most likely correspond to Syntrophomonas sp. and D. latus. As visible in Figure S2a, there is an association between the bacterial and archaeal morphotypes, in particular between Syntrophomonas and 
Methanogenium, while Desulfobacter clusters were less frequent in all the samples analyzed. DAPI staining observations underline that these three microorganisms are the key players and almost all the cells are active in the SMTZ enrichment culture (Figure S2b). Overall, we can speculate about a syntrophic relationship between these three microorganisms, in which Syntrophomonas converts $15.8 \mathrm{mM}$ butyrate to $\sim 32 \mathrm{mM}$ acetate and $30 \mathrm{mM} \mathrm{H}_{2}$, and $D$. latus consumes $\sim 4 \mathrm{mM}$ acetate coupled to reduction of $\sim 5 \mathrm{mM}$ sulfate and M. cariaci consumes $\sim 24 \mathrm{mM} \mathrm{H}_{2}$ to produce $6 \mathrm{mM} \mathrm{CH}_{4}$ (Table $1)$.

The remaining bacterial phylotypes affiliated with Bacteroidetes, Sulfurovum, Synergistetes, Anaerolineae (Figure 2 and Table Sl). These phylotypes are commonly found in marine sediments (Parkes et al., 2014; Webster et al., 2011; Webster et al., 2010; Fry et al., 2008) and are associated with the decomposition of carbohydrates and proteinaceous substrates (Yamada and Sekiguchi, 2009; Kleinsteuber et al., 2012; Godon et al., 2005). Therefore, the existence of non-butyrateconverting bacteria in all cultures suggests that they either are involved in the degradation of dead biomass or consumption of intermediates of butyrate degradation. FISH with specific probes or DAPI staining didn't highlight any other morphotype within the Bacteria domain. 
A

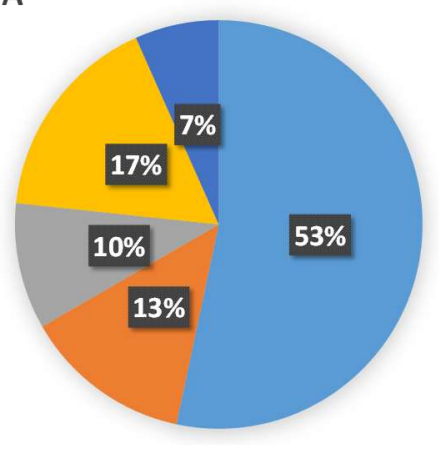

B

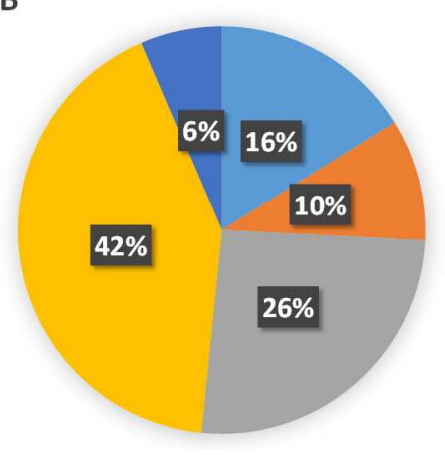

C

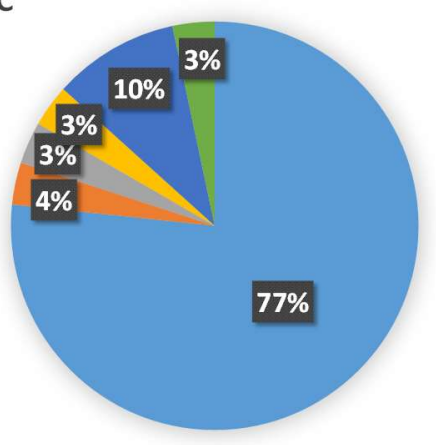

- Syntrophomonas bryantii

- Un. Synergistetes

- Un. Sulfurovum

Un. Bacteroidetes

- Un. Anaerolineae

Un. Anaerolineae

Un. Synergistetes

- Un. Desulfobacter

Un.

Syntrophomonas

Un.

Ignavibacteriales

- Syntrophomonas bryantii

Un. Sulfurovum

- Ralstonia spp.

Un.

Syntrophomonas

- Un. Bacteroidetes
D

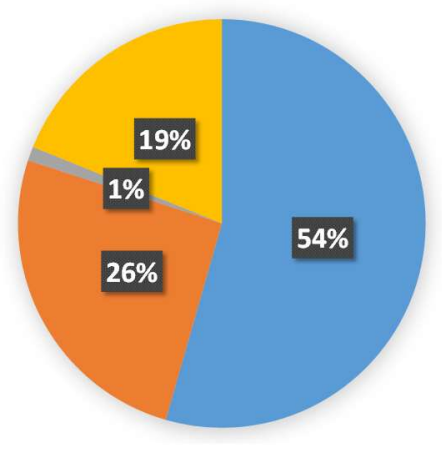

Methanogenium marinum

- Methanosarcina semesiae

- Methanogenium organophilum

- Un.Methanoculle us
E



Methanogenium cariaci

- Methanosarcina lacustris
F

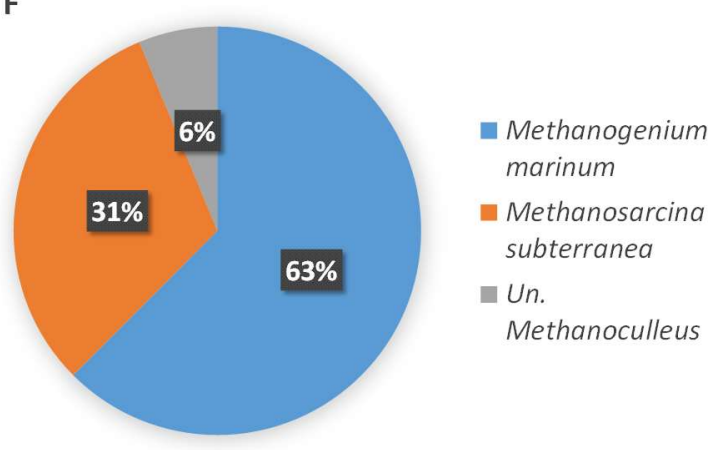

Figure 2. Distribution of bacteria (a, b, c) and archaea (d, e, f) detected in 16S rRNA gene clone libraries derived from butyrate-amended SZ (a, d), SMTZ (b, e) and MZ (c, f) cultures, respectively. Un: Uncultured.

Archaeal clone libraries of the SZ, SMTZ and MZ enrichment cultures show that most of the sequences affiliated with different Methanomicrobiaceae species, members of which are specialists in $\mathrm{H}_{2-}$ and formate-utilization (Garcia et al., 2006) (Figure 2d, 2e, $2 \mathrm{f}$ and Table S2). Hydrogenotrophic methanogens are commonly found in near-surface marine sediments (Parkes et al., 2007; Sørensen et al. 1981; Blair and Carter, 1992). In the SMTZ, most clones (110 clones, 98\%) 
representing Methanomicrobiaceae affiliated with Methanogenium cariaci (Fig 2e, Table S2). SZ and MZ cultures were dominated by clones affiliated with Methanogenium marinum (54\%). The other methanogenic phylotype found both in SZ and MZ cultures was an uncultured Methanoculleus sp., showing 92-95\% similarity to its closest cultured species Methanoculleus taiwanensis (Table S2). The fact that this phylotype has low similarity to any cultured species, suggests that it may represent a novel species within the family Methanomicrobiaceae. Both Methanogenium and Methanoculleus species use $\mathrm{H}_{2}$ and formate for methane production and were isolated from marine sediments (Romesser et al. 1979; Chong et al., 2002; Weng et al., 2015). Methanogenium sp. and Methanoculleus sp. consumed $\mathrm{H}_{2}$ /formate that was produced by the conversion of butyrate and acted as syntrophic partners of Syntrophomonas in the MZ and SZ enrichments (Fig ld, lf). This is supported by the amount of methane produced which is close to the stoichiometric values of syntrophic butyrate degradation in SZ and MZ cultures (Table 1). The relationship of Methanogenium sp. and Methanoculleus sp. with Syntrophomonas was reflected in FISH micrographs of SZ and MZ cultures (Figures S1 and S3). Archaeal cells are more abundant than bacterial cells in the SZ culture (Fig S1). The other methanogenic species detected in all butyrateconverting enrichment cultures was Methanosarcina sp. with 26\%, 2\% and 31\% of the clones in the SZ, SMTZ and MZ clone libraries, respectively (Figure 2 and Table S2). The closest cultured relative of Methanosarcina sp. was M. semesiae in the SZ culture, M. lacustris in the SMTZ culture and M. subterranea in the MZ culture (Table S2). Methanosarcina species are metabolically diverse and able to consume methylated compounds, acetate and $\mathrm{H}_{2} / \mathrm{CO}_{2}$ (Kendall and Boone, 2006b). M. semesiae and $M$. subterranea species grow only on methyl compounds, but not on acetate, $\mathrm{H}_{2} / \mathrm{CO}_{2}$ or formate (Lyimo et al., 2000; Shimizu et al., 2015). However, the enrichment cultures did not contain any methyl group substrates, instead acetate, $\mathrm{H}_{2} / \mathrm{CO}_{2}$ or formate were the only possible methanogenic substrates. Therefore, Methanosarcina-related phylotypes found in these cultures might have consumed these intermediates (Fig ld, lf). FISH micrograph of MZ culture showed that Methanosarcina sp. cells were clustered with Syntrophomonas sp. cells, suggesting that Methanosarcina sp. may be syntrophic partners of Syntrophomonas sp. (Figure S3). However, this should be clarified with further research employing specific probes.

The initial sediment slurries from which SZ and SMTZ enrichment cultures were derived were dominated by sequences related to Methanosaetaceae and contained much less sequences affiliated to Methanosarcina (Chapter 3). After 4 sub-culturing steps Methanosaetaceae-related sequences were lost and Methanosarcinaceae-related methanogens were further enriched in the SZ 
culture (Figure 2 and Table S2). In the SMTZ enrichment culture the aceticlastic methanogens were not enriched (Table S2). Instead, an acetotrophic sulfate-reducer (Desulfobacter) became the dominant acetate-consuming microorganism.

\subsubsection{Propionate conversion}

Propionate conversion was studied under methanogenic conditions in a similar manner as butyrate conversion (Table 1, Figure 3). Maintenance of activity in the propionate-converting enrichment cultures was more difficult compared to the butyrate-converting cultures. After each transfer, propionate conversion started after a long lag phase. The only culture in which propionate conversion started immediately was the SZ culture and propionate was consumed faster as compared to the SMTZ and MZ cultures (Figure 3). In the SZ culture, $18.2 \mathrm{mM}$ propionate was completely converted to $24.5 \mathrm{mM}$ methane in 70 days (Table 1). The acetate originating from the inoculum $(1.8 \mathrm{mM})$ was consumed completely by day 35 . Thereafter, $0.8 \mathrm{mM}$ acetate was produced at the end of the incubation period and consumed by day 70 (Table 1). Propionate conversion in the MZ cultures was considerably slower than propionate conversion in the SZ culture (Figure 3c). $17.9 \pm 0.01 \mathrm{mM}$ propionate was completely, but slowly converted to $26.4 \pm 1.6 \mathrm{mM}$ methane within 297 days. The acetate detected in the beginning of the incubation was consumed within the first 36 days. Thereafter, $1.5 \pm 0.0 \mathrm{mM}$ acetate was produced and consumed the end of the incubation period (Table 1). The amount of methane ultimately produced from propionate in the SZ (1.3 mol of methane/propionate) and in the MZ cultures (1.5 mol of methane/propionate) was close to the value predicted from the stoichiometry of the methanogenic propionate conversion pathway $\left(\mathrm{CH}_{3} \mathrm{CH}_{2} \mathrm{COO}^{-}+1.75 \mathrm{H}_{2} \mathrm{O} \rightarrow 1.75 \mathrm{CH}_{4}+1.25 \mathrm{HCO}_{3}{ }^{-}+0.25 \mathrm{H}^{+}\right)$(Chapter 4-Table 1, reactions 1+5+6).

During the incubation, $\mathrm{H}_{2}$ and formate were not detected and only traces of acetate were intermediately found. Propionate conversion in methanogenic environments is a rate limiting step and complete propionate conversion can be accomplished only if products are consumed by partner organisms (Schink and Stams, 2013; McInerney et al., 2008). The stoichiometry of propionate conversions in SZ and MZ cultures shows that the formation of the products is in pace with their formation from propionate (Fig 3d, 3f). 

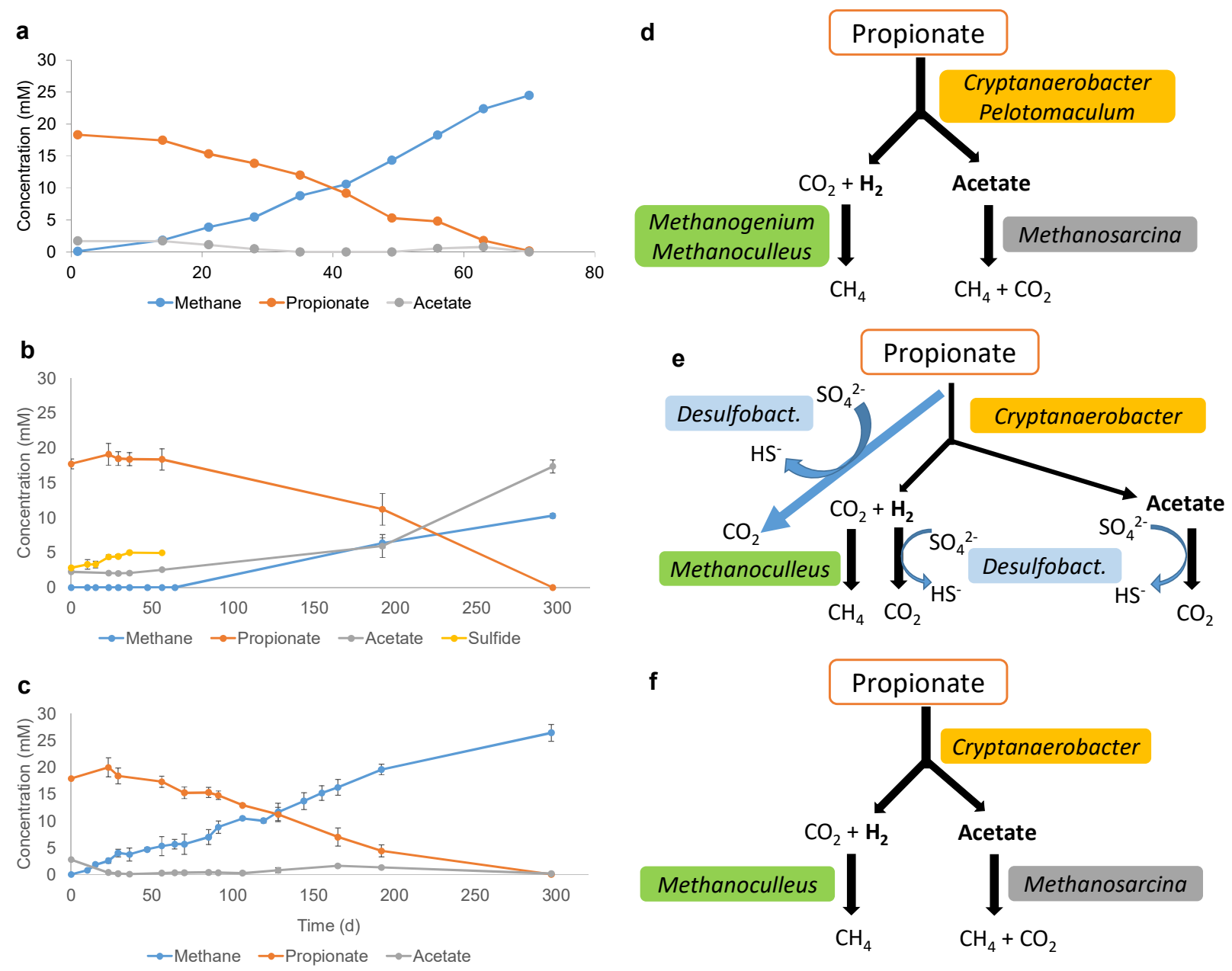

Figure 3. Propionate conversions (SZ (a), SMTZ (b) and MZ (c)) and proposed propionate conversion routes (SZ (d), SMTZ (e) and MZ (f)) in the cultures. Propionate conversion routes are proposed based on microorganisms with high relative abundance and observed propionate conversion stoichiometries. Values are mean of biological duplicates for SMTZ and MZ. As the replicate cultures of SZ grew slow, the values from only one replicate is used in the graph. Error bars indicate the standard deviations. Desulfobact. = Desulfobacterium.

The propionate conversion rate in SMTZ culture was similar to that in the MZ culture (0.06 $\mathrm{mM} /$ day). The conversion dynamics in SMTZ culture differed significantly from the SZ and MZ cultures (Figure 3). After a lag phase of more than 56 days propionate conversion proceeded by concomitant acetate and methane production. Here, $17.7 \pm 0.70 \mathrm{mM}$ propionate was converted to $10.3 \pm 0.35 \mathrm{mM}$ methane and $15.1 \pm 0.95 \mathrm{mM}$ acetate (Figure $3 \mathrm{~b}$ and Table 1 ). This indicated that the conversion of propionate occurred by coupling of acetogenesis and hydrogenotrophic methanogenesis processes (Fig 3e). The ratio of consumed propionate to the produced acetate and methane in the SMTZ cultures is $1: 0.9: 0.6$. As the theoretical ratio is $1: 1: 0.75$, this suggests that 
aceticlastic methanogenesis did not occur, and a minor part of the hydrogen/formate may have been used by sulfate reducers (Fig 3e).

\subsubsection{Microbial community in propionate-degrading enrichments}

Bacterial clone library analysis of propionate-amended enrichment cultures carried out on samples taken after 70 days for the SZ culture and 297 days for the SMTZ and MZ cultures, revealed dominance of clones related to Cryptanaerobacter sp. in the SZ (54\% of all sequences) and MZ (35\% of all sequences) cultures (Figure $4 \mathrm{a}, 4 \mathrm{c}$ ). C. phenolicus is the only cultured species of the genus Cryptanaerobacter and it degrades phenol in syntrophic association with hydrogenotrophic methanogens (Juteau et al., 2005). The closest cultured relatives of two clones in the SZ culture affiliated with Pelotomaculum isophthalicicum (96\%), and Pelotomaculum terephthalicicum (95\%), which are phylogenetically closely related to each other and to C. phenolicus (Ezaki, 2009). P. terephthalicicum and $P$. isophthalicicum are known syntrophs that metabolize a variety of phthalate isomers and other aromatic compounds (McInerney et al., 2008; Qiu et al., 2006). As the sequence similarities of Cryptanaerobacter sp. and Pelotomaculum sp. with their closest cultured relatives detected in our study are below the threshold value of novel species status (98.65\%) (Kim et al., 2014), these phylotypes seem to be novel species of propionate-converting bacteria within the family Peptococcaceae. A recent study suggested that Cryptanaerobacter sp. and Pelotomaculum sp. were the propionate-oxidizing species in enrichment cultures inoculated with sludge from agricultural biogas plants (Ahlert et al., 2016). Therefore, further research is needed to test the ability of Cryptanaerobacter sp. to consume propionate.

The majority of the clones ( $47 \%$ of all sequences) obtained from the SMTZ enrichment culture related to Desulfobacterium indolicum (93\% similarity) (Figure $4 \mathrm{~b}$ and Table S3). Additionally, 2 clones in this culture are distantly related to Desulfosarcina variabilis (93\% similarity). As this value is below the suggested threshold (94.5\% 16S rRNA based) for delineating a novel genus (Yarza et al., 2014), this phylotype might be a novel genus with members utilizing propionate and/or acetate within the family Desulfobacteraceae. The other phylotype that is affiliated with the Desulfobacteraceae family in this culture was assigned as Desulfobacterium catecholicum (13\% of all sequences) (Table S3). This sulfate-reducing bacterium can utilize $\mathrm{H}_{2}+\mathrm{CO}_{2}$, formate, acetate and propionate as well as aromatic compounds and alcohols as carbon sources (Szewzyk and Pfennig, 1987). 


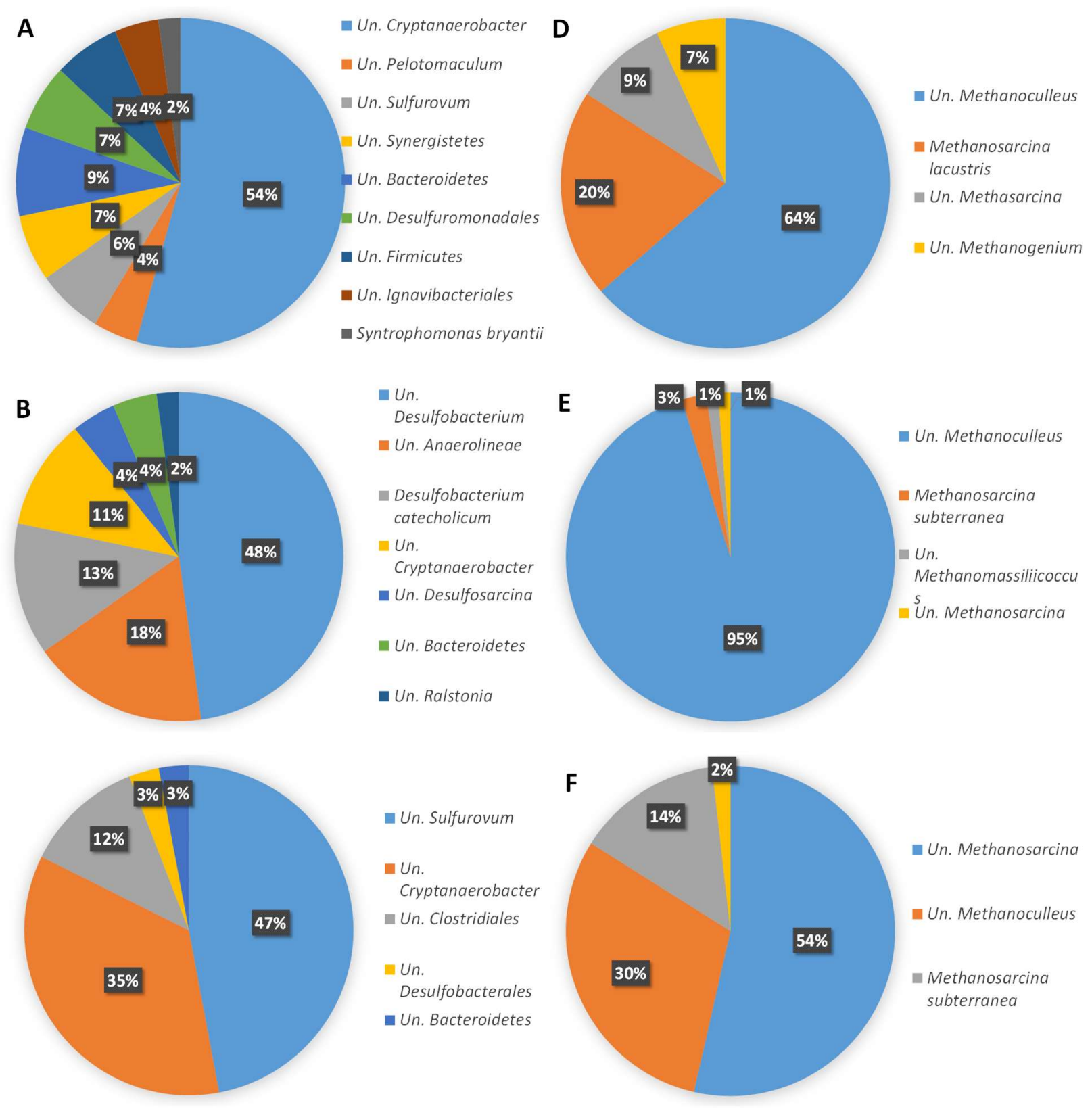

Figure 4. Distribution of bacteria (a, b, c) and archaea (d, e, f) detected in 16S rRNA gene clone libraries derived from propionate-amended SZ (a, d), SMTZ (b, e) and MZ (c, f) cultures, respectively. Un: Uncultured.

Therefore, D. catecholium in the SMTZ enrichment may have degraded propionate and/or contributed to the utilization of $\mathrm{H}_{2} / \mathrm{CO}_{2}$, formate or acetate that are formed during incomplete propionate conversion (Fig 3e). The phylotype related to Cryptanaerobacter phenolicus comprised 
of $11 \%$ of all sequences in SMTZ culture. Its existence after several sub-culturing steps suggests that this bacterium is possibly involved in syntrophic propionate conversion (Fig 3e).

The majority of the bacterial clones retrieved from MZ enrichment culture showed 96-97\% similarity (16S rRNA based) to Sulfurovum aggregans, belonging to Epsilonproteobacteria. This is followed by uncultured Cryptanaerobacter sp., representing 35\% of the clones (Figure 4c and Table S3). S. aggregans is a mesophilic chemolithoautotrophic bacterium isolated from a deep-sea hydrothermal vent (Mino et al., 2014). This bacterium uses hydrogen as electron donor, $\mathrm{CO}_{2}$ as carbon source and sulfur, thiosulfate or nitrate as electron acceptors, the ability to use sulfate as an electron acceptor has not been reported. Epsilonproteobacteria have been shown to be widely present in marine and terrestrial environments (Campbell et al., 2006) and detected in anoxic saline sediment incubations fed with different electron donors (Koizumi et al., 2005), suggesting that they can use a wide range of electron donors and acceptors and/or constitute relationships with other metabolic groups of bacteria (Campbell et al., 2006). The function of the Sulfurovum sp. in SZ and MZ cultures is hard to define based on the physiological characterization of the closest relative of this phylotype. Nevertheless, we cannot rule out the possibility that the Epsilonproteobacteria in our culture might have used hydrogen and acetate as electron donor. Starke et al (2016) have reported that an epsilonproteobacterial Campylobacterales member featured the fastest and highest acetate incorporation in the microbial consortium containing benzene-degrading Cryptanaerobacter, Pelotomaculum species. Similarly, Sulfurovum-like species were suggested to be the hydrogen- or acetate-consuming syntrophic partners of Pelotomaculum, Cryptanaerobacter phylotypes during the degradation of benzene under sulfate-reducing conditions (Kleinsteuber et al., 2008; Hermann et al., 2010). Epsilonproteobacterial species have also been observed in phenol-degrading methanogenic reactors, however their role remained unexplored (Ju and Zhang, 2014; Zhang et al., 2005; Fang et al., 2004). Therefore, Sulfurovum sp. in our culture might be responsible for hydrogen and/or acetate consumption. As sulfate reduction by Sulfurovum sp. has not been reported, the possible electron acceptor for Sulfurovum sp. in MZ culture may be oxidized sulfur compounds (e.g. sulfur, thiosulfate). These compounds might have been formed by the reaction of hydrogen sulfide and traces of oxygen that were possibly introduced into the bottle during sampling.

The majority of all archaeal clones obtained from 3 propionate-amended cultures (125 out of 184) affiliated with an uncultured Methanoculleus. 106 clones derived from the SZ and SMTZ cultures have 95-96\% 16S rRNA based similarity to M. taiwanensis (Figure 4d, 4e and Table S4). 
This uncultured Methanoculleus phylotype constituted 64\% and 95\% of the SZ and SMTZ clones, respectively. The MZ culture yielded 16 clones (29\% of the MZ archaeal clones) highly related to Methanoculleus marisinigri (98\% 16S rRNA based). This indicates that Methanoculleus formed close relationship with the propionate-converting bacteria in the cultures and consumed $\mathrm{H}_{2}$ produced during incomplete propionate conversion (Fig 3d, 3e, 3f). The second most prevalent sequences (40 out of 184) in the archaeal clone libraries affiliated with Methanosarcina sp. (32 clones) and Methanosarcina subterranea (Figure 4 and Table S4). These clones were dominant in the MZ culture (38 out of 56) and in the minority in SMTZ culture (2 out of 84 clones). The observed complete propionate conversion to methane and $\mathrm{CO}_{2}$ in the $\mathrm{MZ}$ culture strongly suggests that Methanosarcina sp. has been involved in acetate utilization in the cultures (Fig 3f).

Samples for FISH were collected at the beginning of the incubation period (day 16) only from MZ cultures. In Fig. S4, fluorescence micrographs of samples hybridized with probes specific for Archaea (red) and Bacteria (green) reveal cells with morphology resembling Methanosarcina and Methanculleus. For the bacteria, no distinct morphotype could be recognized due to a lack of cultured representatives (Fig 3, Table 1).

\subsection{Conclusions}

Long term slurry incubations and sub-culturing resulted in highly enriched syntrophic cultures converting propionate and butyrate to methane. Propionate was converted completely by syntrophs, aceticlastic and hydrogenotrophic methanogens under methanogenic conditions, whereas acetate accumulation occurred during butyrate conversion and aceticlasts were not present in the enrichments. Microbial diversity analysis revealed dominance of Syntrophomonas sp. in butyrate-amended and Cryptanaerobacter sp. in propionate-amended enrichment cultures. The detected Cryptanaerobacter sp. may be a novel propionate-converting syntroph within the family Peptococcaceae, as it is only distantly related to its closest relatives. Low sulfate-amended butyrate- and propionate-converting SMTZ cultures contained sequences related to Desulfobacteraceae family members besides presumed syntrophs. Hydrogenotrophic methanogens Methanogenium and Methaoculleus acted as the main syntrophic partners in butyrate- and propionate-converting cultures, respectively, and aceticlastic methanogens consumed acetate especially in propionate-converting cultures. Acetate in the sulfate-amended cultures was consumed by sulfate reducers, pointing to a competitive advantage of sulfate reducers over methanogens in the presence of sulfate. Overall, the results of our study indicated the presence of 
a functionally competent syntrophic community in the coastal marine sediment of Aarhus Bay, capable to degrade propionate and butyrate to methane under methanogenic conditions at different biogeochemical zones. Further attempts to obtain the novel marine propionate- and butyrate-converting syntrophs, Syntrophomonas sp. and Cryptanaerobacter sp., in defined cultures are in progress.

\section{Acknowledgments}

We gratefully acknowledge Monika Jarzembowska and Ton van Gelder (Wageningen University \& Research) for technical assistance. This work has been funded by the Wimek Graduate School of Wageningen University \& Research and the Darwin Center for Biogeosciences (the Netherlands). 


\section{Supplementary materials}

Table S1. Overview of bacterial clones sequenced and their similarity with their closest relatives in butyrate-amended SZ, SMTZ and MZ cultures.

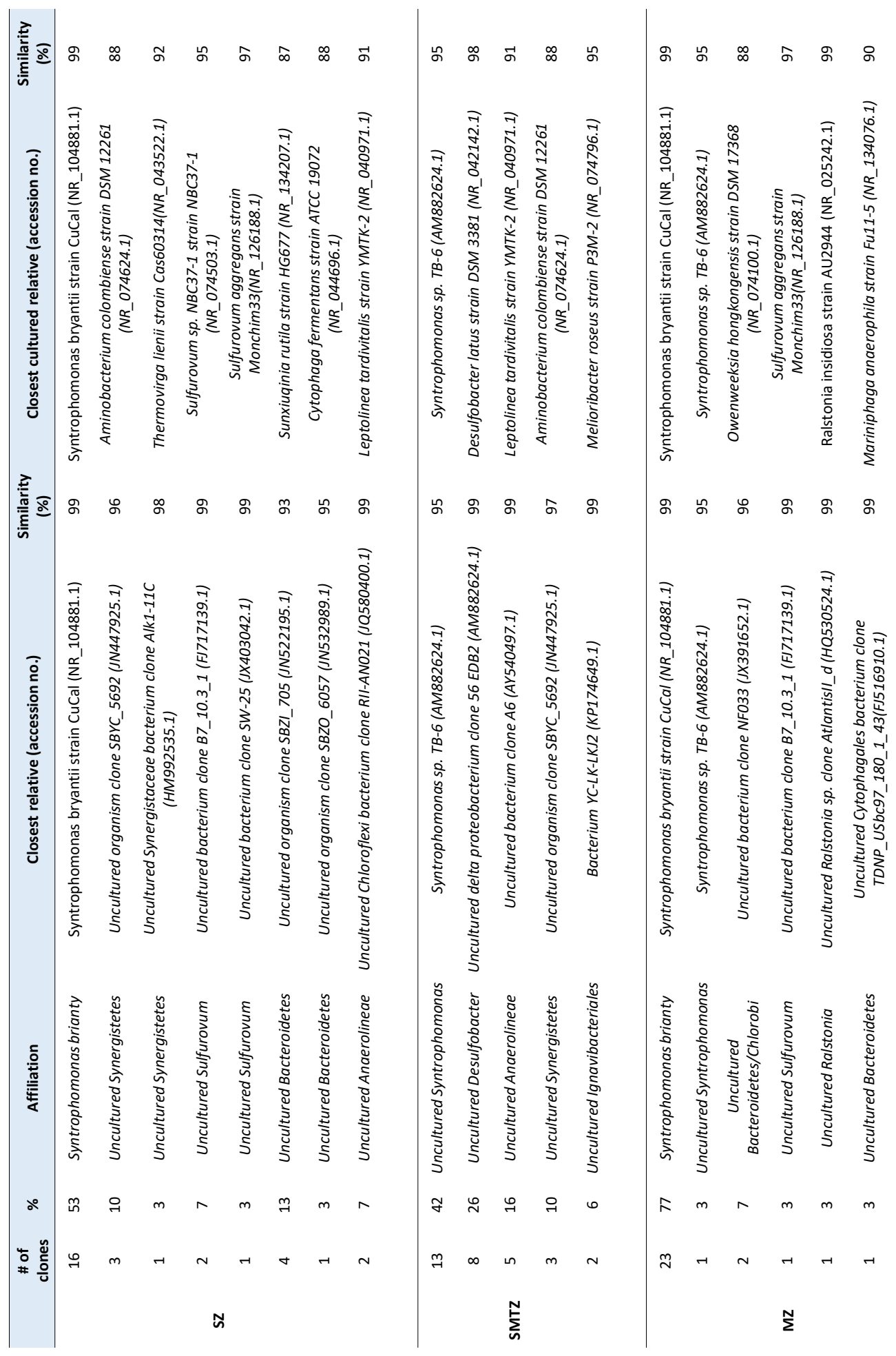


Table S2. Overview of archaeal clones sequenced and their similarity with their closest relatives in butyrate-amended SZ, SMTZ and MZ cultures.

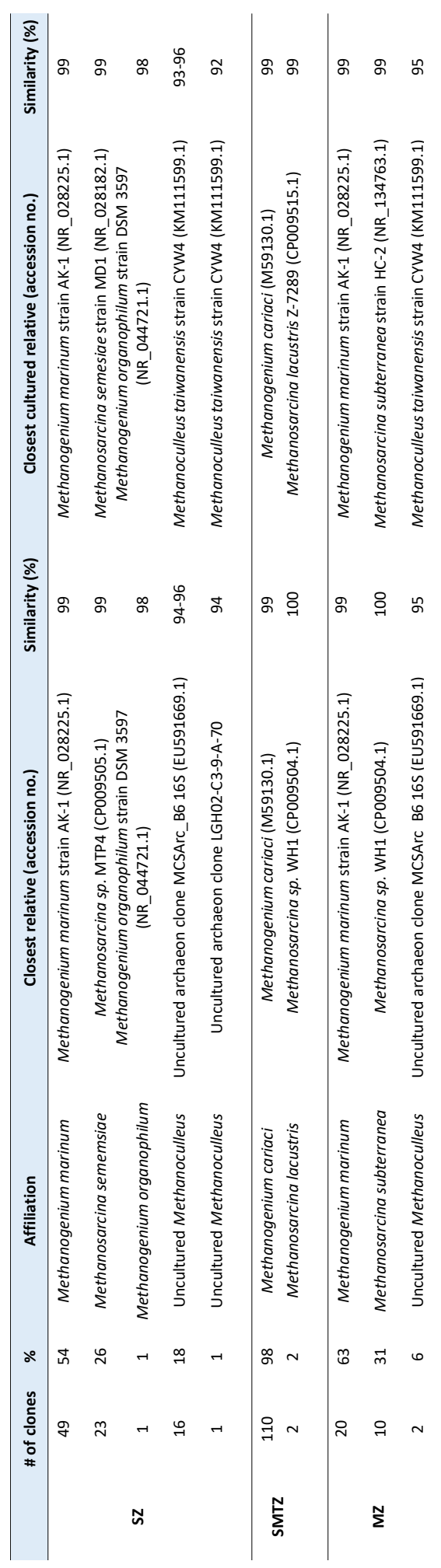


Table S3. Overview of bacterial clones sequenced and their similarity with their closest relatives in propionate-amended SZ, SMTZ and MZ cultures.

\begin{tabular}{|c|c|c|c|c|c|c|c|}
\hline & \# of clones & $\%$ & Affiliation & Closest relative (accession no.) & $\begin{array}{c}\text { Similarity } \\
(\%)\end{array}$ & Closest cultured relative (accession no.) & $\begin{array}{c}\text { Similarity } \\
(\%)\end{array}$ \\
\hline \multirow{24}{*}{ SZ P1 } & 10 & 22 & $\begin{array}{c}\text { Uncultured } \\
\text { Cryptanaerobacter }\end{array}$ & Uncultured bacterium clone PLAB4 (AB701662.1) & 98 & $\begin{array}{l}\text { Cryptanaerobacter phenolicus strain LR7.2 16S } \\
\text { (NR_025757.1) }\end{array}$ & 96 \\
\hline & & & Uncultured & & & Cryptanaerobacter phenolicus strain LR7.2 16S & \\
\hline & 6 & 13 & Cryptanaerobacter & Uncultured bacterium clone ARWH-BF05 (AB546032.1) & 98 & (NR_025757.1) & 92-96 \\
\hline & & 0 & $\begin{array}{l}\text { Uncultured } \\
\text { Cryptanaerobacter }\end{array}$ & & & Cryptanaerobacter phenolicus strain LR7.2 16S & \\
\hline & & & Uncultured & & & Cryptanaerobacter phenolicus strain LR7.2 16S & \\
\hline & 5 & 11 & Cryptanaerobacter & Uncultured Firmicutes bacterium clone NPA01 (AB853918.1) & 98 & (NR_025757.1) & 96 \\
\hline & & & & & & Sulfurovum aggregans strain Monchim 33 & \\
\hline & 3 & 7 & Uncultured Sulfurovum & Uncultured bacterium clone B7_10.3_1 (FJ717139.1) & 99 & (NR_126188.1) & 97 \\
\hline & 3 & 7 & Uncultured Synergistetes & Uncultured organism clone SBXZ_3880 (JN433166.1) & 91 & $\begin{array}{l}\text { Aminobacterium colombiense strain DSM } 12261 \\
\text { (NR_074624.1) }\end{array}$ & 91 \\
\hline & 3 & 7 & $\begin{array}{c}\text { Uncultured } \\
\text { Desulfuromonadales }\end{array}$ & Desulfuromusa bakii strain Gyprop (NR_026175.1) & 99 & Desulfuromusa bakii strain Gyprop (NR_026175.1) & 99 \\
\hline & & & & & & Cytophaga fermentans strain ATCC 19072 & \\
\hline & 2 & 4 & Uncultured Bacteroidetes & Uncultured bacterium clone b49 (KJ578036.1) & 99 & (NR_044696.1) & 90 \\
\hline & 2 & 4 & Uncultured Bacteroidetes & Uncultured bacterium clone Er-MLAYS-71 (EU542481.1) & 99 & $\begin{array}{c}\text { Prolixibacter bellariivorans strain JCM } 13498 \\
\text { (NR 113041.1) }\end{array}$ & 89 \\
\hline & & & & & & Gracilibacter thermotolerans strain JW/YJL-S1 & \\
\hline & 1 & 2 & Uncultured Firmicutes & Uncultured Gracilibacter sp. clone 2_100_B10_b (JQ087078.1) & 97 & (NR_115692.1) & 97 \\
\hline & 1 & 2 & Uncultured Firmicutes & Uncultured bacterium clone B19CH1_61_65 (HF558553.1) & 97 & Proteiniborus ethanoligenes strain GW (NR_044093.1) & 87 \\
\hline & 1 & 2 & Uncultured Firmicutes & Uncultured bacterium clone HS006 (JX391342.1) & 99 & Tissierella creatinini strain BN11 (NR_104805.1) & 94 \\
\hline & & & & & & Pelotomaculum terephthalicicum strain JT & \\
\hline & 1 & 2 & Uncultured Pelotomaculum & Uncultured bacterium clone 347_TC26_111 (KM251003.1) & 99 & (NR_040948.1) & 95 \\
\hline & 1 & 2 & Uncultured Pelotomaculum & Uncultured bacterium clone II (AB091325.1) & 96 & Pelotomaculum isophthalicicum strain JI (NR_041320.1) & 96 \\
\hline & & & & Uncultured Ignavibacteriales bacterium clone HYIII51_Bac16s_AQDS01_01_B01 & & & \\
\hline & 1 & 2 & Uncultured Ignavibacteriales & (KU324269.1) & 96 & Melioribacter roseus strain P3M-2 (NR_074796.1) & 84 \\
\hline & 1 & 2 & Uncultured Ignavibacteriales & Uncultured Chlorobi bacterium clone FII-AN013 1 (JQ579954.1) & 99 & Melioribacter roseus strain P3M-2 (NR_074796.1) & 0.9 \\
\hline & 1 & 2 & Syntrophomonas bryantii & Syntrophomonas bryantii strain CuCal (NR_104881.1) & 99 & Syntrophomonas bryantii strain CuCal (NR_104881.1) & 99 \\
\hline \multirow{13}{*}{ SMTZ P2 } & 22 & 47 & $\begin{array}{c}\text { Uncultured } \\
\text { Desulfobacterium }\end{array}$ & ncultured Desulfobacteraceae clone TDNP USbc97 20480 (FI516922.1) & & Desulfobacterium indolicum strain In04 (NR 028897.1) & $93-94$ \\
\hline & 7 & 15 & $\begin{array}{l}\text { Desulfobacterium } \\
\text { Uncultured Anaerolineae }\end{array}$ & $\begin{array}{l}\text { Uncultured Chloroflexi bacterium clone RII-AN021 (JQ580400.1) } \\
\text { (J).1) }\end{array}$ & $\begin{array}{l}99 \\
99\end{array}$ & $\begin{array}{l}\text { Desulfobacterium indolicum strain In04 (NR_028897.1) } \\
\text { Leptolinea tardivitalis strain YMTK-2 (NR_040971.1) }\end{array}$ & $\begin{array}{c}93-94 \\
91\end{array}$ \\
\hline & & & Desulfobacterium & & & [Desulfobacterium] catecholicum strain NZva20 & \\
\hline & 6 & 13 & catecholicum & [Desulfobacterium] catecholicum strain NZva20 (NR_028895.1) & 99 & (NR_028895.1) & 99 \\
\hline & 5 & 11 & $\begin{array}{c}\text { Uncultured } \\
\text { Cryptanaerobacter }\end{array}$ & Uncultured bacterium clone A_Lac-1_35 (EU307089.1) & 98 & $\begin{array}{l}\text { Cryptanaerobacter phenolicus strain LR7.2 } \\
\text { (NR_025757.1) }\end{array}$ & 95 \\
\hline & 2 & 4 & Uncultured Bacteroidetes & Uncultured bacterium clone Er-MLAYS-71 (EU542481.1) & 99 & Prolixibacter denitrificans strain MIC1-1 (NR_137212.1) & 89 \\
\hline & 1 & 2 & Uncultured Desulfosarcina & Uncultured delta proteobacterium clone 81 EDB3 (AM882635.1) & 99 & $\begin{array}{l}\text { Desulfosarcina variabills strain } \\
\text { (NR_044680.1) }\end{array}$ & 93 \\
\hline & & & & & & Desulfosarcina variabilis strain Montpellier & \\
\hline & 1 & 2 & Uncultured Desulfosarcina & Uncultured delta proteobacterium clone 19 EDB1 (AM882602.1) & 99 & (NR_044680.1) & 98 \\
\hline & 1 & 2 & Uncultured Anaerolineae & Uncultured Chloroflexi bacterium clone RII-AN043 (JQ580422.1) & 99 & Leptolinea tardivitalis strain YMTK-2 (NR_040971.1) & 91 \\
\hline & & & & & & & \\
\hline & 1 & 2 & Desulfuromonadales & Pelobacter sp. SFB93, complete genome (CP015519.1) & 96 & Pelobacter sp. SFB93, complete genome (CP015519.1) & 96 \\
\hline & 1 & 2 & Uncultured Ralstonia & Uncultured Ralstonia sp. clone Atlantisll_d (HQ530524.1) & 99 & Ralstonia insidiosa strain ATCC 49129 (CP016023.1) & 99 \\
\hline \multirow{15}{*}{ MZ P3 } & 14 & 41 & $\begin{array}{l}\text { Uncultured Sulfurovum } \\
\text { Uncultured }\end{array}$ & Uncultured bacterium clone B7_10.3_1 (FJ717139.1) & 99 & $\begin{array}{l}\text { Sulfurovum aggregans strain Monchim33 } \\
\text { (NR_126188.1) } \\
\text { Cryptanaerobacter phenolicus strain LR7.2 }\end{array}$ & 96-97 \\
\hline & 7 & 21 & $\begin{array}{l}\text { Cryptanaerobacter } \\
\text { Uncultured }\end{array}$ & Uncultured bacterium clone A_Lac-1_35 (EU307089.1) & 98 & $\begin{array}{c}\text { (NR_025757.1) } \\
\text { Cryptanaerobacter phenolicus strain LR7.2 }\end{array}$ & 95 \\
\hline & 3 & 9 & Cryptanaerobacter & Uncultured bacterium clone: PLAB4 (AB701662.1) & 98 & (NR_025757.1) & 96 \\
\hline & & & & & & Gracilibacter thermotolerans strain JW/YJL-S1 & \\
\hline & 2 & 6 & Uncultured Clostridiales & Uncultured bacterium clone 300_TC38_31 (KM250983.1) & 98 & (NR_115692.1) & 97 \\
\hline & & & Uncultured & & & Cryptanaerobacter phenolicus strain LR7.2 & \\
\hline & 2 & 6 & Cryptanaerobacter & Uncultured bacterium clone: ARWH-BF05 (AB546032.1) & 98 & (NR_025757.1) & 96 \\
\hline & & & & & & Sulfurovum aggregans strain Monchim 33 & \\
\hline & 1 & 3 & Uncultured Sulfurovum & Uncultured bacterium clone B1_10.3_2 (FJ717148.1) & 99 & $\begin{array}{l}\text { (NR_126188.1) } \\
\text { Sulfurovum agareagns strain Monchim } 33\end{array}$ & 97 \\
\hline & 1 & 3 & Uncultured Sulfurovum & Uncultured bacterium clone T3-1_246 (KX097819.1) & 99 & (NR_126188.1) & 98 \\
\hline & 1 & 3 & $\begin{array}{l}\text { Uncultured Bacteroidetes } \\
\text { Uncultured }\end{array}$ & Uncultured bacterium clone N0047 (JX391483.1) & 94 & $\begin{array}{c}\text { Tangfeifania diversioriginum strain G22 (NR_134211.1) } \\
\text { Desulfatiferula olefinivorans strain LM2801 }\end{array}$ & 93 \\
\hline & 1 & 3 & Desulfobacterales & Uncultured bacterium zdt-33i5 clone zdt-33i5 (AC150251.1) & 96 & 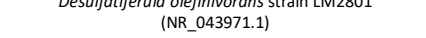 & 86 \\
\hline & & & & & & Acetomicrobium faecale strain DSM 20678 & \\
\hline & 1 & 3 & Uncultured Clostridiales & Uncultured bacterium clone B19CH1_61_65 (HF558553.1) & 97 & (NR_117173.1) & 87 \\
\hline & 1 & 3 & Uncultured Clostridiales & Uncultured bacterium clone LI142-3110 (FJ672228.1) & 98 & Garciella nitratireducens strain Met 79 (NR_025688.1) & 91 \\
\hline
\end{tabular}


Table S4. Overview of archaeal clones sequenced and their similarity with their closest relatives in propionate-amended SZ, SMTZ and MZ cultures.




Figure S1. FISH images with probes EUB338 mix (green, Bacteria) and ARC915 (red, Archaea), from the butyrate-amended SZ culture after 16 days of cultivation. In (a), arrows indicate the morphologies possibly related with the clonal analysis data. Cells of Methanoculleus sp. are little coccoids, Methanogenium sp. are big irregular cocci and Synthophomonas sp. are rods. In (b) DAPI staining of the same microscopic field demonstrates the probes coverage of total cells. Scale bar is $5 \mu \mathrm{m}$.

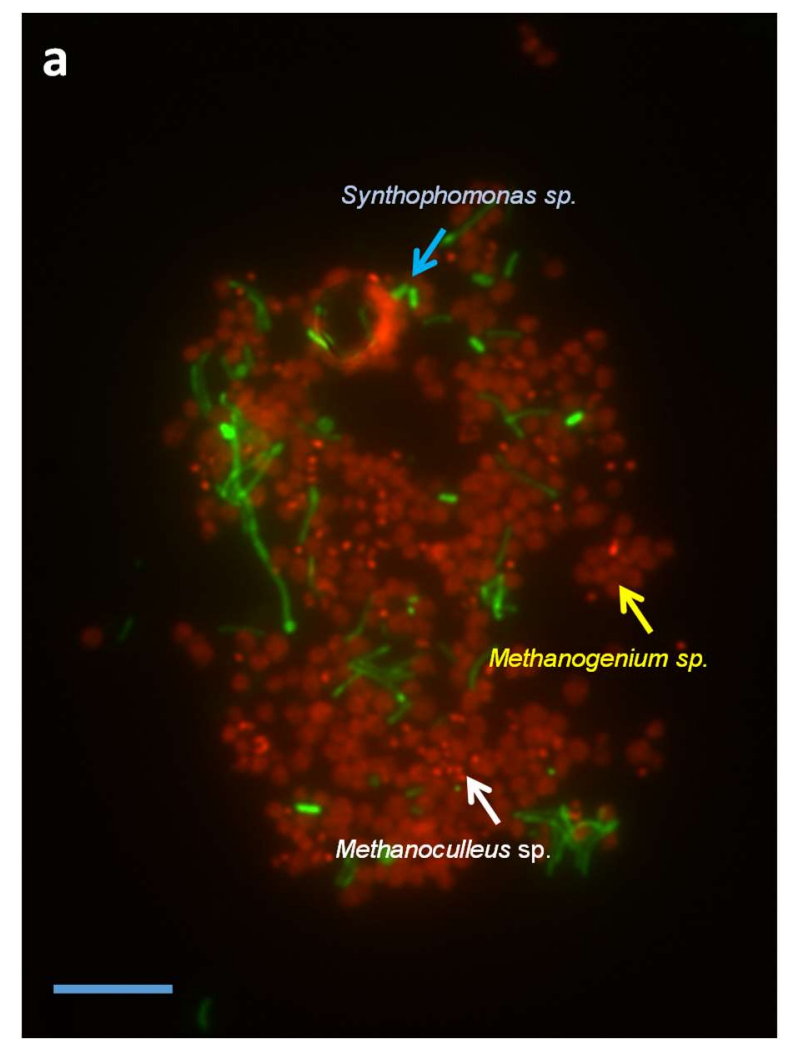

\section{b}


Figure S2. FISH images with probes EUB338 mix (green, Bacteria) and ARC915 (red, Archaea), from the butyrate-amended SMTZ culture after 16 days of cultivation. In (a), arrows indicate morphologies possibly related with the clonal analysis data. In (b), DAPI staining of the same microscopic field demonstrates the probes coverage of total cells. Scale bar is $5 \mu \mathrm{m}$.
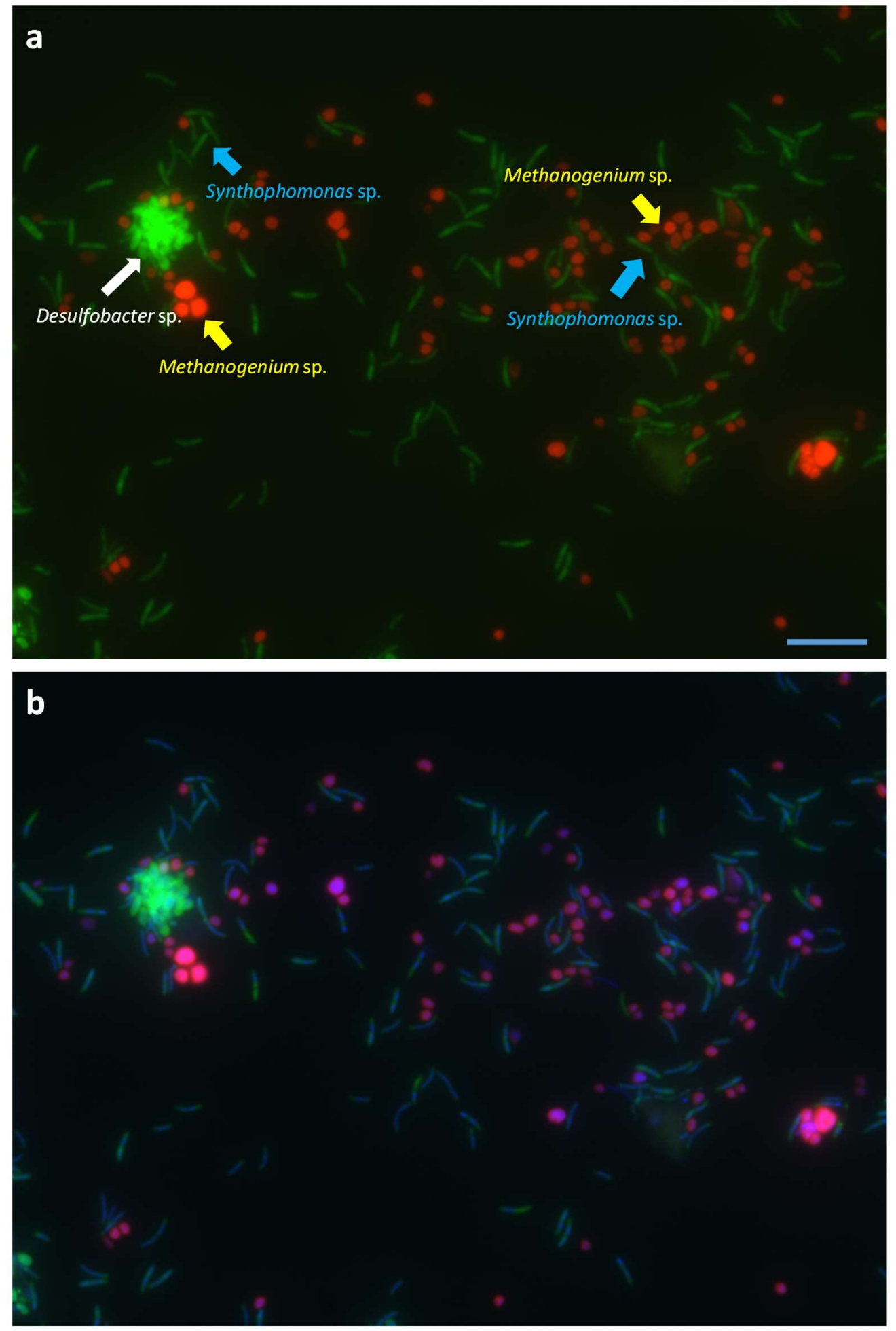
Figure S3. FISH images with probes EUB338 mix (green, Bacteria) and ARC915 (red, Archaea), from the butyrate-amended MZ culture after 16 days of cultivation. In all the images, arrows indicate the morphologies possibly related with the clonal analysis data. In (a) a general overview of the culture, (b) a cluster of Syntrophomonas and Methanosarcina cells, (c) close association between Syntrophomonas and Methanogenium cells, (d) DAPI staining of the same microscopic field shown in figure $S_{3}$. Scale bar $5 \mu \mathrm{m}$.

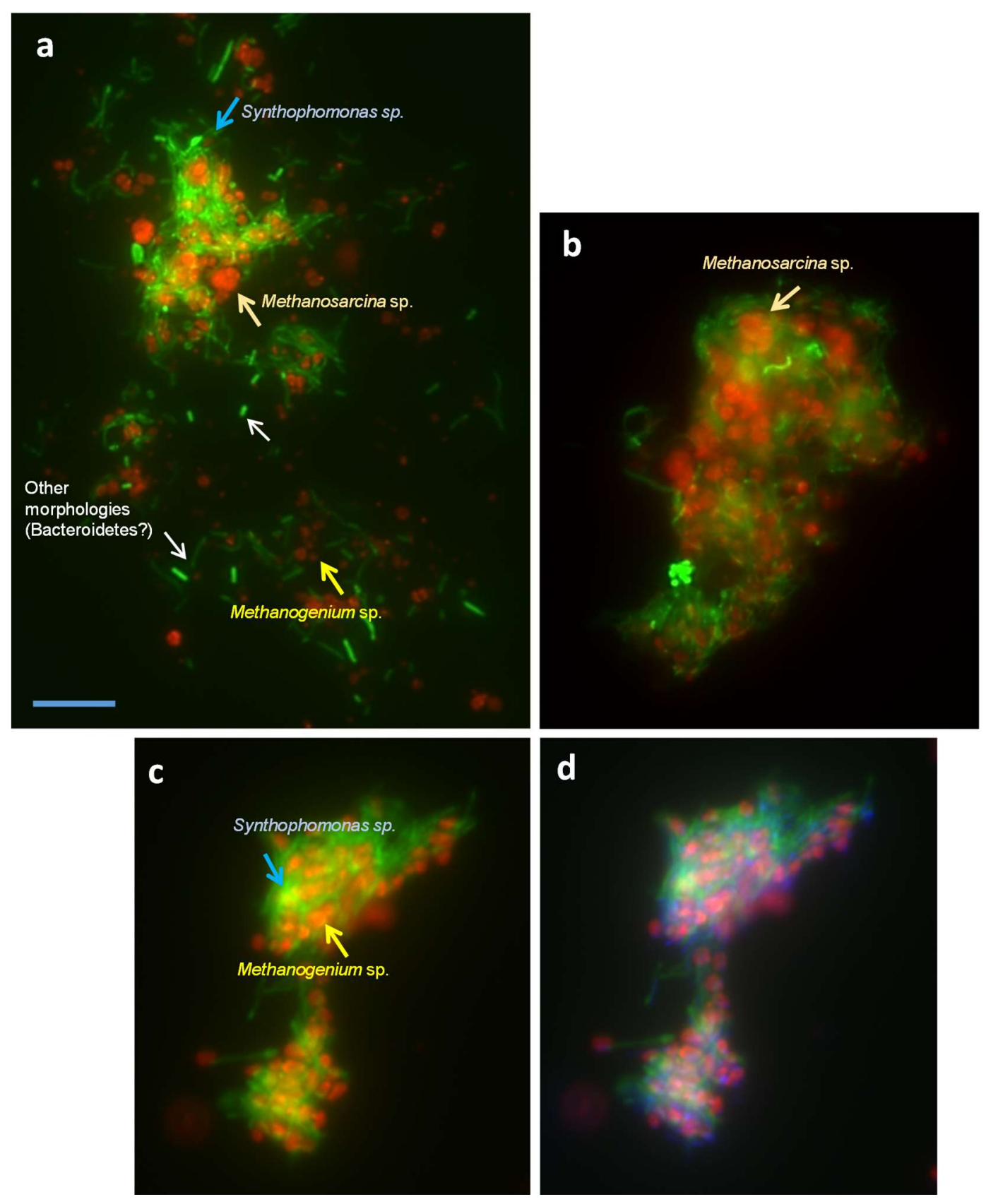


Chapter 6

Figure S4. FISH images with probes EUB338 mix (green, Bacteria) and ARC915 (red, Archaea), from the propionate-amended MZ culture after 16 days of cultivation. Scale bar $5 \mu \mathrm{m}$.
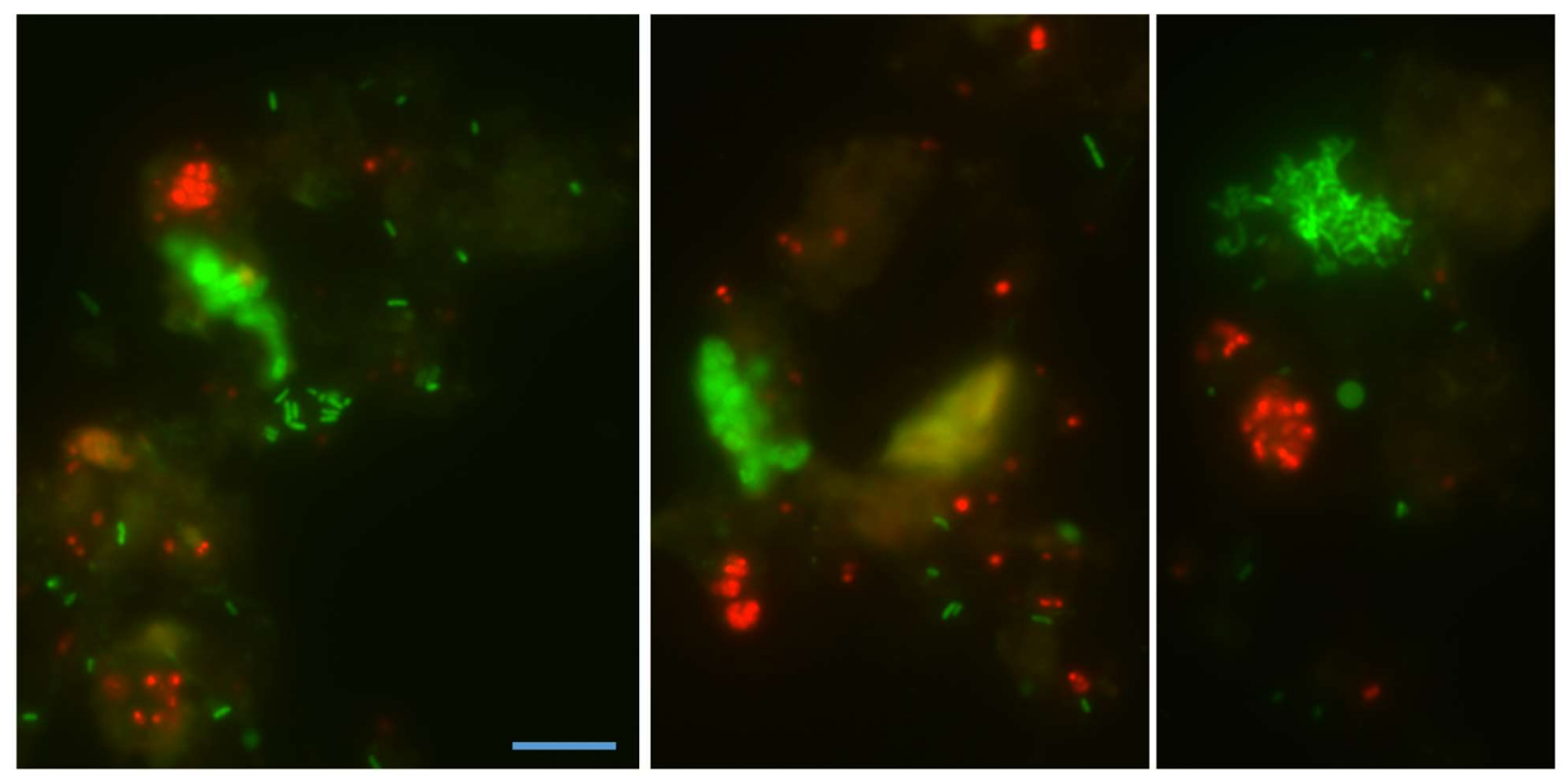


\section{Chapter 7}

\section{General Discussion}

\section{Derya Özüölmez}


Coastal marine ecosystems receive regular inputs of organic matter and nutrients from primary production of phytoplanktons, rivers, coastal erosion, and the atmosphere (Jørgensen, 1982). In coastal marine sediments, the oxic zone is a thin layer (few $\mathrm{mm}$ or $\mathrm{cm}$ ) due to the rapid aerobic mineralization and therefore oxygen is limited. Because of that the major part of the organic matter is degraded in the anoxic part of the sediment involving physiologically different microbes. Decomposition of organic matter is carried out by combined action of different metabolic groups of bacteria, including primary fermenters, secondary fermenters, bacteria that reduce different electron acceptors such as nitrate, iron, sulfate or carbon dioxide and at least two types of methanogenic archaea. Major intermediates of anaerobic decomposition of organic matter such as acetate, propionate, butyrate and $\mathrm{H}_{2}$ are the most important electron donors for sulfate-reduction in marine environments. Thus sulfate-reducing bacteria (SRB) contribute significantly to the mineralization of organic matter as well as to the Earth's sulfur cycle (Jørgensen, 1982; Sørensen et al., 1981). It is estimated that $25-50 \%$ of the organic carbon is mineralized through sulfate reduction, which makes sulfate an important electron acceptor in anoxic part of the marine sediments (Jørgensen, 1982). The conversion of propionate and butyrate in deep sulfate-depleted sediments is critical due to the lack of electron acceptors other than $\mathrm{CO}_{2}$. For complete degradation of propionate or butyrate, syntrophic cooperation of one acetogenic bacteria and two methanogenic archaea is required (Schink and Stams, 2013) (Chapter 1). Syntrophic associations between propionate- or butyrate-degrading bacteria and methanogens contribute substantially to methane production in marine sediments (Kendall et al., 2006). Previous studies have shown SRB predominance in high-sulfate and methanogen predominance in low-sulfate environments (Ward and Winfrey, 1985; Widdel, 1988; Cappenberg, 1974; Mountfort and Asher, 1979). However, several other studies demonstrated that methanogenesis occurs simultaneously with sulfate reduction in sulfate-containing anoxic sediments (Oremland and Taylor, 1978; Oremland and Polcin, 1982; Oremland et al., 1982a, 1982b, 1987; Kiene et al., 1986; Oremland et al., 1988; Wang and Lee, 1995) and active SRB in methane-rich anoxic sediments (Jørgensen, 1978; Thomsen et al., 2001; Leloup et al., 2007, 2009).

\subsection{Conversion of propionate and butyrate in marine sediments}

\subsubsection{Propionate and butyrate conversion coupled to sulfate reduction}

The effect of sulfate on butyrate and propionate conversion was studied in chapter 3 and 4 . The sulfate in all slurries was rapidly consumed showing that sulfate reduction is a dominant process 
for butyrate and propionate conversion in Aarhus Bay sediments. Rapid sulfate reduction in methane zone sediment slurries indicates an active sulfate-reducing community in the sulfatedepleted sediments (Jørgensen, 1978; Thomsen et al., 2001; Leloup et al., 2007, 2009). These findings confirm findings of previous studies showing the presence of active SRB at similar cell numbers in the upper and lower parts of the coastal marine sediments of Limfjorden and Aarhus Bay, Denmark (Leloup et al., 2009; Thomsen et al., 2001; Jørgensen, 1978) and deep-sea sediment (Leloup et al., 2007).

Butyrate and propionate can only be metabolized by syntrophic associations of acetogenic bacteria and hydrogen- and acetate-consuming methanogens in the absence of sulfate (Muyzer and Stams, 2008). The results obtained in the chapter 3 and 4 show that the absence of sulfate causes a delay in butyrate and propionate conversion, which requires cooperation of different microorganisms (McInerney et al., 2008). Acetate, which was accumulated by syntrophic conversion of propionate and butyrate, was consumed rapidly and methane was produced concomitantly. This suggests the presence of active syntrophic propionate- and butyrateconverting microorganisms and their ability to form consortia with hydrogen and acetate utilizing methanogens throughout the sediment column. Our results are consistent with previous studies that showed evidence of butyrate- and propionate-converting syntrophs in sulfate-containing marine sediments (Kendall et al., 2006; Kendall and Boone, 2006a).

The trend of product formation and consumption during butyrate and propionate conversions was considerably different in high $(20 \mathrm{mM})$ and low $(3 \mathrm{mM})$ sulfate amended SMTZ slurries at $25^{\circ} \mathrm{C}$. High concentration of sulfate caused low and stable methane yields despite a sudden methane increase at the end of the incubation period (Chapter 3 and 4). Lower methane concentrations (339-4183 $\mu \mathrm{mol} / \mathrm{slurry})$ in high sulfate amended slurries as compared to low sulfate amended slurries (15509-73569 $\mu \mathrm{mol} / \mathrm{slurry})$ along slurry incubations indicates possible sulfide inhibition on methanogenesis (Pender et al., 2004; O’Flaherty et al., 1998; Shin et al., 1995). Under low sulfate condition, however, methane concentrations were relatively high, especially during butyrate conversion. The results obtained from the chapters 3 and 4 indicate that low sulfate concentration stimulates the whole microbiome; both sulfate reduction and methanogenesis occur in Aarhus Bay sediments. 


\subsubsection{The effect of temperature on propionate and butyrate conversion}

Different temperatures have an impact on butyrate and propionate conversion in Aarhus Bay sediments. The results of chapter 3 and 4 show that at low incubation temperature $\left(10^{\circ} \mathrm{C}\right)$ butyrate and propionate conversion is very slow, with low sulfate consumption and high acetate accumulation. This result is in line with previous experiments showing decreased sulfate reduction rates at temperatures below $25^{\circ} \mathrm{C}$ in sulfate-amended sediment slurries (Weston and Joye, 2005). On the other hand, efficient acetate consumption with simultaneous methane production in sulfate-free slurries at $25^{\circ} \mathrm{C}$ has also been shown by Roussel et al (2015).

Sulfate-methane transition zone is located between sulfate and methane zones and characterized by low sulfate and methane concentrations. In this sediment zone, methane and sulfate are mutually depleted by the microbial anaerobic oxidation of methane (AOM) (Sultan et al., 2016; Iversen and Jørgensen, 1985). Methanogenesis during butyrate and propionate conversion at $10^{\circ} \mathrm{C}$ in high-sulfate amended SMTZ slurries was relatively low and fluctuating (Chapter 3 and 4). Although high sulfate and sulfide concentrations can be the reason of low methane yields, the fluctuating methane production during butyrate conversion and total methane consumption during propionate conversion can be related to anaerobic oxidation of methane (AOM). AOM has been reported to occur in SMT zone of Aarhus Bay (Webster et al., 2011; Aquilina et al., 2010; Thomsen et al., 2001; Dale et al., 2008). Since the incubation temperature $\left(10^{\circ} \mathrm{C}\right)$ resembles the in situ temperature of Aarhus Bay (Dale et al., 2008) the microbial communities residing in SMTZ might be involved in oxidation of the produced methane.

\subsection{Microbial community dynamics in propionate and butyrate conversion}

Microbial communities in the butyrate and propionate converting enrichment slurries show different dynamics throughout the several biogeochemical zones (SZ, SMTZ, MZ).

\subsubsection{Bacterial community at different sulfate concentrations}

The sulfate concentrations caused a variation of presence of intact polar membrane lipids (IPL), which are the building blocks of cell membranes and representative of living biomass, in butyrateconverting slurries (Chapter 5), although the microbial composition changed considerably both in propionate and butyrate-converting slurries (Chapter 3 and 4). The enriched bacteria in the incubations with sulfate were similar when butyrate and propionate were used as substrate. These 
were mainly the sulfate reducers belonging to Desulfobacteraceae and Desulfobulbaceae (Figure 1 and 2). Desulfosarcina and Desulfobacterium dominated the butyrate-converting slurries, whereas Desulfosarcina, Desulfobulbus and Desulforhopalus were the main SRB in propionate-converting slurries. The increase in the relative abundance of Desulfobacteraceae and Desulfobulbaceae in SZ, SMTZ and MZ sediment slurries suggests the presence of sulfate reducers throughout the anoxic sediment column. Our findings confirm the reports showing the abundance and the activity of sulfate-reducing bacteria in Aarhus Bay sediments at Station 1 (Leloup et al. 2009) and Station 6 (Sahm et al., 1999), in Kysing Fjord, Denmark, (Thomsen et al., 2001) and Mariager Fjord, Denmark (Wagner et al., 2005). The ability of some SRB to grow fermentatively may explain their abundance in sulfate-depleted sediments and the sudden increase in their relative abundance in sulfateamended methane zone slurries.

The absence of sulfate causes a change in the enriched microbial community. Syntrophomonas increased in relative abundance in the butyrate-converting sediment slurries, whereas Cryptanaerobacter dominated the propionate-converting sediment slurries. Syntrophomonas is a known butyrate-degrading syntrophic specialist that grows in close association with methanogens and other hydrogen- and/or formate-utilizing microorganisms (Plugge et al., 2011; Sousa et al., 2009; Oude Elferink et al., 1994). The fact that the relative abundance of Syntrophomonas was also high in some sulfate-containing slurries and kept the high abundance after several transfers (Chapter 6), lead us to speculate that the presence of sulfate can prevent Syntrophomonas species to be the dominant bacterial group, but does not inhibit their involvement in butyrate conversion, suggesting a role of syntrophic butyrate conversion even in the presence of sulfate. Coexistence of Syntrophomonas spp. and sulfate reducers were reported during butyrate (Struchtemeyer et al., 2011; Visser et al., 1993) and oleate and palmitate (Sousa et al., 2009) degradation in the presence of sulfate.

The genus Cryptanaerobacter belongs to the family Peptococcaceae. As Cryptanaerobacter dominated the slurries that contain low or no sulfate (Chapter 4) (Figure 7.2) and the enrichment cultures after subculturing process (Chapter 6), we hypothesize that a novel species of this bacterium might have the capability to convert propionate in syntrophy with hydrogenotrophic methanogens. The idea behind this hypothesis is based on ; i) the isolation of $C$. phenolicus from a methanogenic consortium, ii) the syntrophic lifestyle of its closest cultured relatives and iii) the presence of propionate as a sole carbon source in the slurries. 


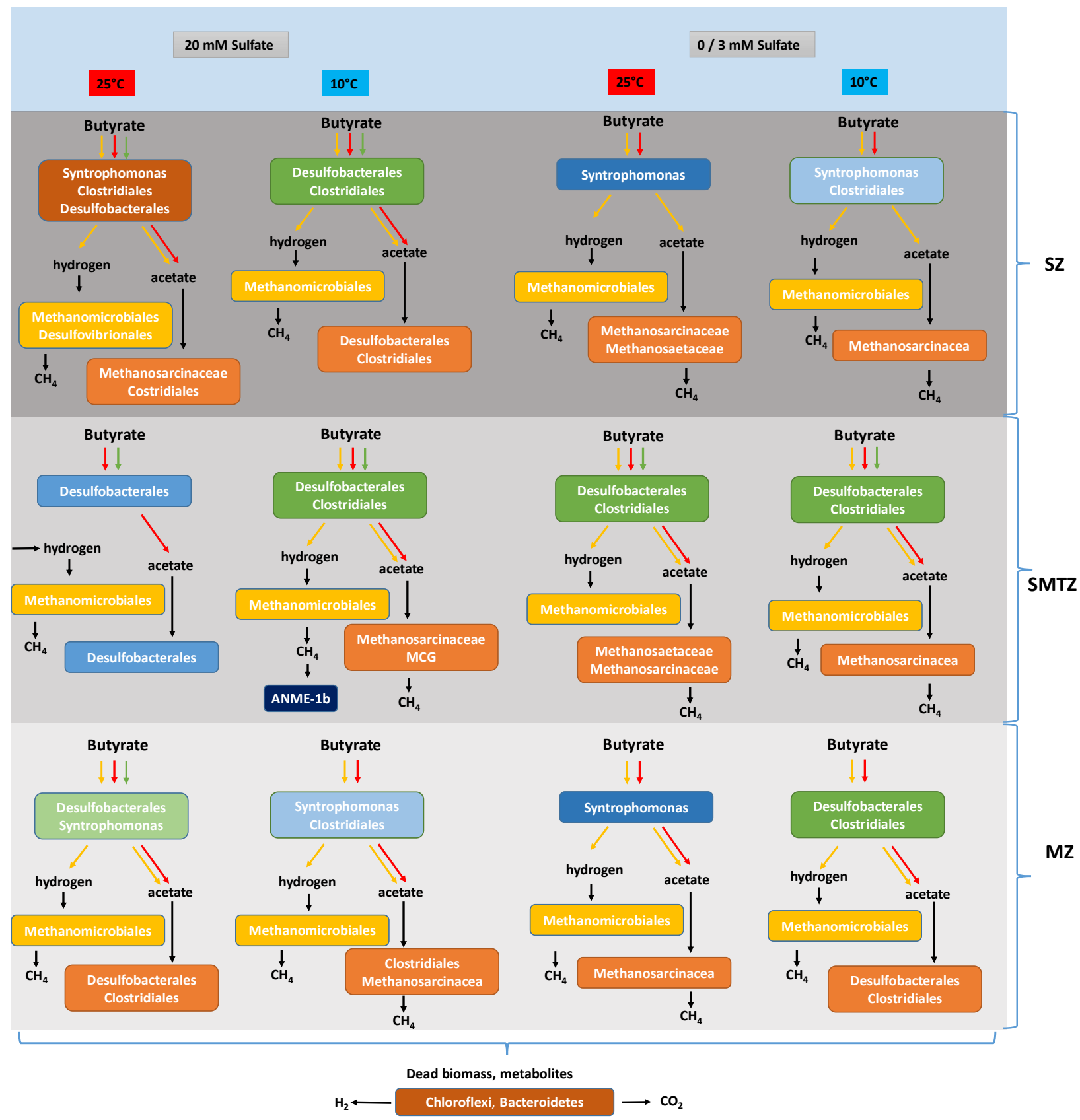

Figure 1. Overview of butyrate conversion and the proposed responsible microbial community at different temperatures and sulfate concentrations in enrichment slurries of sulfate, sulfate-methane transition and methane zone sediment of Aarhus Bay. Possible butyrate conversion pathways are shown with different coloured arrows; red arrows represent incomplete butyrate conversion coupled to sulfate reduction, green arrows represent complete butyrate conversion coupled to sulfate reduction, yellow arrows represent syntrophic butyrate conversion. Horizontal arrow represents the substrates originate from fermentation, decomposition of dead biomass and/or metabolites. 
Additionally, the hypothesis is supported with the statistical analysis showing positive correlation of the order Clostridiales with methane production and temperature. Desulfobacteraceae was dominant under sulfate-free and low-sulfate conditions during butyrate and propionate conversion (Chapter 4). This result agrees with the previous studies reporting that non-sulfate reducing, syntrophic members of Desulfobacteraceae involve in butyrate (Kendall et al., 2006) and benzene degradation (Ulrich and Edwards, 2003). Despite the importance of Syntrophobacter in syntrophic propionate conversion (Plugge et al., 2011) in shallow methanogenic sediments (Lloyd et al., 2006; McInerney et al., 2008), we did not detect Syntrophobacter in Aarhus Bay sediment or in the sediment slurries. Similarly, Leloup et al. (2009) reported only very low abundance of Syntrophobacter-like sequences at Station 1 in Aarhus Bay. The results of this thesis illustrate the significance of metabolic flexibility of microorganisms under changing conditions such as temperature, electron acceptor availability, the presence/absence of partner organisms.

\subsubsection{Bacterial community at different temperatures}

The most obvious difference in the microbial community composition at high and low temperature is the dominance of Cryptanaerobacter at $25^{\circ} \mathrm{C}$, and Desulfobacteraceae (Desulfofaba), especially Desulfobulbaceae members (Desulfobulbus, Desulforhopalus) at $10^{\circ} \mathrm{C}$ during propionate conversion. Likewise, Desulfobulbaceae and some Desulfobacteraceae members are negatively correlated with temperature (Chapter 4). Incubation at low temperature had also an impact on IPL composition in propionate-converting slurries (Chapter 5). In butyrate-converting slurries, there is no clear difference in microbial community between different temperatures. However, Clostridiales had higher relative abundance at $10^{\circ} \mathrm{C}$ regardless of the sulfate concentration and the sediment depth. Clostridiales are commonly found in surface and deep marine sediments, soils and methanogenic environments (Fry et al., 2008; Parkes et al., 2014). As $10^{\circ} \mathrm{C}$ resembles the in situ temperature, our results provide support for possible involvement of Clostridiales in butyrate conversion in marine sediments.

\subsubsection{Bacterial community at different sediment depth}

Pyrosequencing of 16S rRNA gene amplicons of Aarhus Bay sediment revealed the dominance of the Desulfobacteraceae in the SMTZ, accounting for 79\% of the sequences (Chapter 3 and 4 ) in the original sediment. This result shows that the microbial community in the SMTZ is distinct from the 
microbial community in the SZ and MZ. This is in accordance with previous studies documenting the high abundance of Desulfobacteraceae in SMTZ of marine sediments (Wagner et al., 2005).

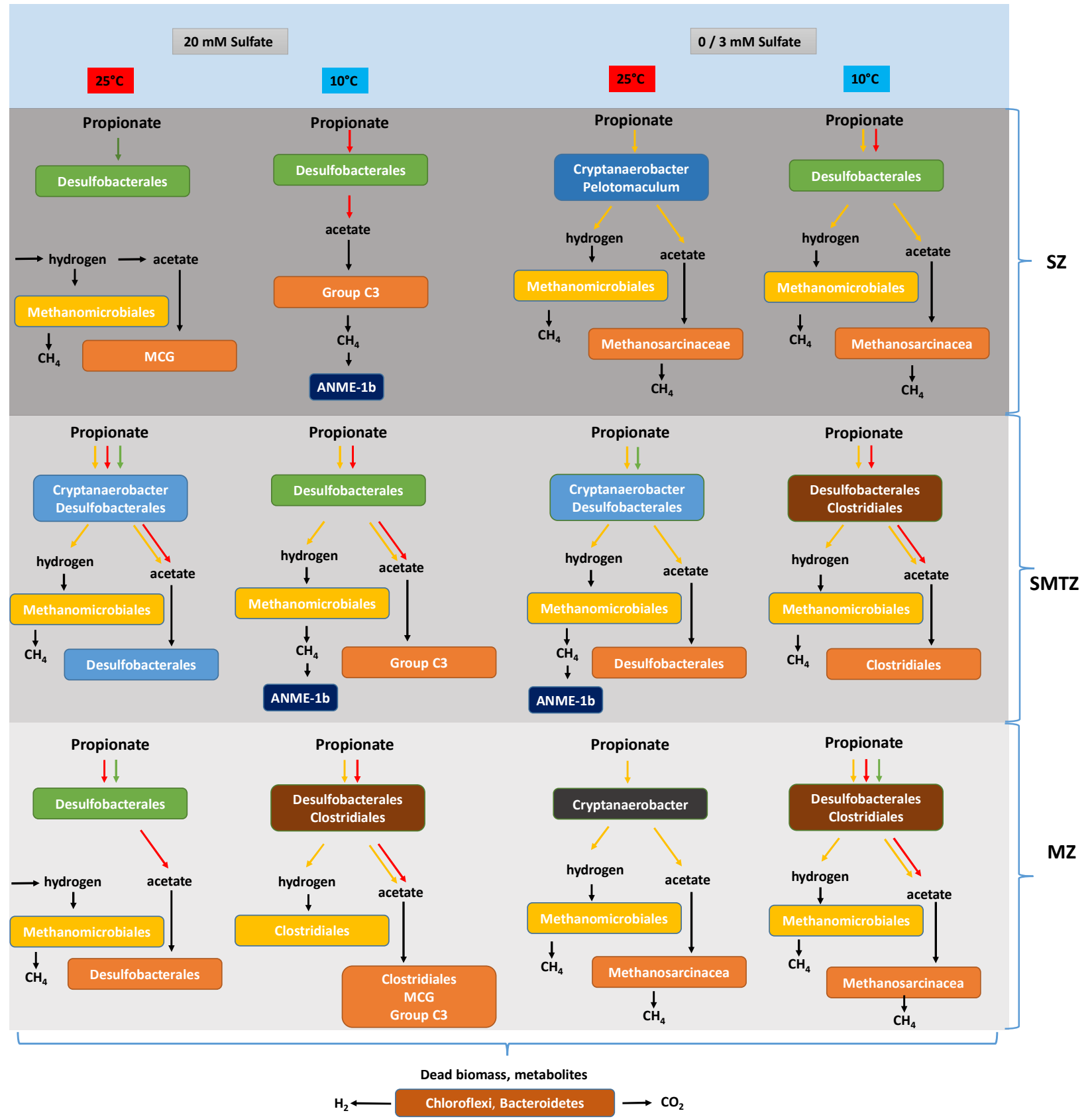

Figure 2. Overview of propionate conversion and the proposed responsible microbial community at different temperatures and sulfate concentrations in enrichment slurries of sulfate, sulfate-methane transition and methane zone sediment of Aarhus Bay. Possible propionate conversion pathways are shown with different coloured arrows; red arrows represent incomplete propionate conversion coupled to sulfate reduction, green arrows represent complete propionate conversion coupled to sulfate reduction, yellow arrows represent syntrophic propionate conversion. Horizontal arrow represents the substrates originate from fermentation, decomposition of dead biomass and/or metabolites. 
Pyrosequencing showed that Desulfobacteraceae was still present in the SMTZ enrichment slurries after long term incubation and therefore might have involved in propionate and butyrate conversion at high and low sulfate concentrations. In Chapter 6, Syntrophomonas became dominant in the low sulfate containing SMTZ slurry at $25^{\circ} \mathrm{C}$ after several transfers, and Desulfobacter latus, belonging to Desulfobacteraceae, was the syntrophic partner of Syntrophomonas. On the other hand, the dominant propionate-converting organism at the same incubation condition was still a member of Desulfobacteraceae (Chapter 6). This suggests that Syntrophomonas can effectively compete with sulfate reducers for butyrate in the presence of sulfate.

The presence of Desulfobacteraceae, including acetate-degrading sulfate reducers, at $10^{\circ} \mathrm{C} \mathrm{might}$ be associated with their syntrophic interaction with methane-oxidizing archaea, similar to the in situ condition (Knittel and Boetius, 2009; Boetius et al., 2000). This result is consistent with low and fluctuating methane concentrations in high sulfate-amended butyrate- and propionateconverting SMTZ slurries in which the abundance of ANME-1b (methane oxidizing archaea, ANME) is relatively high (Chapter 3 and 4 ).

\subsubsection{Archaea as syntrophic partners}

Methanomicrobiales was the dominant archaeal order in both butyrate- and propionate-converting slurries regardless of the origin of the sediment, the incubation temperature and the sulfate concentration (Chapter 3 and 4). This finding is supported by the statistical analysis showing that Methanomicrobiales clustered with various IPLs that were abundant at different temperatures and sulfate concentrations in the butyrate- and propionate-converting slurries (Chapter 5). Similarly, different genera of Methanomicrobiaceae were dominant in the enrichment cultures after several transfers (Chapter 6). They were the main syntrophic partners of butyrate and propionate degraders responsible for $\mathrm{H}_{2}$ and/or formate consumption. In addition, the FISH micrographs of butyrate-converting enrichment cultures visualize the close relationship of syntrophic bacteria and methanogenic archaea (Chapter 6). The predominance of hydrogenotrophic methanogens has been observed in near-surface and sulfate-rich marine sediments (Webster et al., 2009; Parkes et al., 2007; Kendall and Boone, 2006a; Parkes et al., 1990; Blair and Carter 1992; Sørensen et al. 1981) which explains the high abundance of Methanomicrobiales under sulfidogenic as well as methanogenic conditions in our incubations. 
The other syntrophic partner organisms belonged to the aceticlastic methanogenic families, Methanosarcinaceae and Methanosaetaeceae. Methanosarcina, belonging to Methanosarcinaceae, was present in sulfate-amended and sulfate-free slurries whereas Methanosaetaceae was enriched only in slurries with low or free of sulfate (Chapter 3). After subculturing, however, Methanosaetaceae-related methanogens were replaced by Methanosarcinaceae-related methanogens in SZ and by Desulfobacter latus in SMTZ cultures (Chapter 6).

In addition to the methane-producing archaea, methane-oxidizing archaea (ANME-1b) were also detected in propionate and butyrate slurries (Chapter 3 and 4 ), but they were not enriched further, due to their slow growth (Chapter 6). Low methane and high sulfide amounts in SMTZ slurries support the findings of Timmers et al. (2015) where ANME-1b was enriched in batch incubations with Baltic Sea sediments. The enrichment of both ANME-1 and Desulfobacteraceae in SMTZ slurries at low temperature is in accordance with previous findings documenting that SMTZ sediments are dominated by the members of Desulfobacteraceae and ANME clusters, forming close associations and involving in anaerobic oxidation of methane (Harrison et al., 2009; Knittel and Boetius, 2009). The other methane-oxidizing archaeal genera detected in the same slurries with ANME-1b were Methanococcoides and Methanolobus which are defined as ANME-3 Archaea (Knittel and Boetius, 2009, Lösekann et al., 2007, Niemann et al., 2006). Apparently, the incubation condition, inhabiting microbial community and the amounts of sulfate, sulfide and methane in the slurries favoured the enrichment of ANME-3 group.

\section{3 $\mathrm{H}_{2}$ and acetate: substrates for syntrophy or competition?}

The competition mechanism of sulfate reducers and methanogens for hydrogen has been explained by Hoehler et al. (1998). SRB can consume as low as 1-2 $\mathrm{nM} \mathrm{H}_{2}$, while hydrogenotrophic methanogens require a minimum of $10-20 \mathrm{nM} \mathrm{H}_{2}$. However, methanogens can coexist with sulfate reducers in sulfate zone of marine sediments by utilizing methylated compounds such as methanol, methylated amines and methylated sulfides. Nanomolar concentrations of $\mathrm{H}_{2}$ may leak from methanogens which can subsequently be scavenged by sulfate reducers in the methane zone sediments (Finke et al., 2007b). The leaked $\mathrm{H}_{2}$ controls the metabolic processes between different physiological types of organisms.

Energy yields from acetate in sulfate-reducing marine sediment are often sufficiently high to allow several potentially competing microbial processes to co-occur. The main reason is that acetate exists in much higher concentrations than $\mathrm{H}_{2}$, in micromolar concentrations compared to 
nanomolar concentrations of $\mathrm{H}_{2}$ (Albert and Martens 1997; Jørgensen and Parkes 2010), making it theoretically possible for aceticlastic methanogens to co-occur with energetically more favourable acetate-oxidizing sulfate reduction in the sulfate zone (Finke et al., 2007b; Wang et al. 2010b). However, almost all acetate in the sulfate zone is converted to $\mathrm{CO}_{2}$, not to $\mathrm{CH}_{4}$ (Jørgensen and Parkes, 2010), suggesting the predominance of aceticlastic sulfate reduction. Finke et al. (2007b) reported increasing sulfate and decreasing $\mathrm{H}_{2}$ concentrations in marine sediments incubated with methylamine and methanol. They concluded that the $\mathrm{H}_{2}$ production from methanogens becomes favorable under sulfate-reducing conditions and interspecies transfer of leaked $\mathrm{H}_{2}$ during acetate oxidation might occur which provides a mechanism for survival of aceticlastic methanogens in sulfate-reducing sediments (Finke et al., 2007b). The importance of $\mathrm{H}_{2}$ leakage was described for the first time by Phelps et al. (1985) using mixed pure cultures grown on acetate or methanol. They showed sulfate-dependent $\mathrm{H}_{2}$ transfer from Methanosarcina barkeri to Desulfovibrio vulgaris, resulting in less methane production and more $\mathrm{CO}_{2}$ and sulfide production. The methanogens started to produce $\mathrm{H}_{2} / \mathrm{CO}_{2}$ instead of $\mathrm{CH}_{4}$ upon sulfate addition and the sulfate-reducing partner scavenged the produced hydrogen efficiently and kept the concentration low enough for the methanogens to continue producing $\mathrm{H}_{2} / \mathrm{CO}_{2}$. Achtnich et al. (1995) showed that hydrogen-utilizing sulfate reducers influence aceticlastic methanogenesis in anoxic paddy soils leading to oxidation of the methyl carbon. King et al. (1983) reported a decreasing $\% \mathrm{CO}_{2}$ after inhibition of sulfate reduction, thus inhibition of the $\mathrm{H}_{2}$ scavenging partner in marine sediments for non-competitive substrates such as methylamine and trimethylamine. We performed a similar experiment to the study of Phelps et al. (1985) and showed interspecies hydrogen transfer between aceticlastic Methanosaeta concilii and hydrogenotrophic microorganisms, Desulfovibrio vulgaris or Methanococcus maripaludis, using acetate as electron donor (Chapter 2). Our results showed that $D$. vulgaris could reduce sulfate and grow on leaked hydrogen from $M$. concilii, and the cell increase of $M$. maripaludis was synchronized with the growth of $M$. concilii. The switch from methanogenesis to hydrogen production shows cooperation rather than competition between methanogens and sulfate reducers. Hydrogen production from Methanosaeta was demonstrated for Methanosaeta thermophila when growing on acetate (Valentine et al., 200o), and we reported for the first time hydrogen leakage from a mesophilic halotolerant Methanosaeta. In addition to hydrogen leakage, we tested coexistence of $M$. concilii with Desulfobacter latus on acetate under sulfidogenic conditions in mixed pure cultures (Chapter 2). The results showed that acetate conversion by aceticlastic methanogens in the presence of high sulfate and active aceticlastic sulfate reducers is 
possible. Butyrate and propionate conversion at high sulfate concentration occurred by syntrophic interactions of acetogenic bacteria with acetate- and hydrogen-consuming sulfate reducers and methanogens. This was revealed by consumption of accumulated acetate with simultaneous methane production both in sulfate and methane zone slurries (Chapter 3 and 4). Despite cooperative interactions of sulfate reducers and methanogens, we still observed competition between them for acetate. Methanosaetaceae, which was dominant aceticlastic methanogen in enrichment slurries (Chapter 3), was washed out after several transfers and replaced by Methanosarcinaceae in sulfate zone (SZ) cultures and by acetate-consuming D. latus in sulfatemethane transition zone (SMTZ) cultures (Chapter 6). High acetate concentration resulting from incomplete butyrate conversion during sub-culturing process can be the reason of high relative abundance of Methanosarcinaceae, whose members are known to be more successful at high acetate concentrations (Jetten et al., 1992). Additionally, D. latus grew faster in SMTZ cultures, thus gained competitive advantage over methanogens (Muyzer and Stams, 2008).

\subsection{Concluding remarks and future perspectives}

Sulfate reduction and methanogenesis are important terminal electron-accepting processes in coastal marine sediments, and propionate, butyrate, acetate, and $\mathrm{H}_{2}$ /formate are major endproducts of organic matter degradation in these ecosystems. Since the conversion of propionate and butyrate is critical in sulfate-depleted sediments and acetate and $\mathrm{H}_{2} /$ formate play an important role during their degradation, it is important to know which populations of microorganisms are involved in their degradation in the upper and lower parts of the marine sediments and how they interact with each other during degradation.

In this thesis, we demonstrated that sulfate reducers were more competitive than methanogens in high-sulfate environments and thus sulfate reduction is the dominant pathway for butyrate and propionate conversions in Aarhus Bay sediment. Desulfosarcina and Desulfobacterium are involved in butyrate conversion, whereas Desulfosarcina, Desulfobulbus and Desulforhopalus are involved in propionate conversion. We also determined that the presence of SRB, especially Desulfobacteraceae and Desulfobulbaceae, was independent of the presence of sulfate. We observed that Desulforhopalus, SEEP-SRB1 group, unclassified Desulfobacteraceae were more competitive in environments without sulfate and Desulfobulbaceae were more successful at low temperature as compared to Desulfobacteraceae. The fact that many sulfate reducers have both sulfidogenic and acetogenic type of metabolism, increases the chance of survival in environments lacking sulfate. 
The syntrophic butyrate-converting genus Syntrophomonas was detected under sulfate-free as well as low-sulfate conditions, whereas syntrophic conversion of propionate was likely performed by an unknown species of Cryptanaerobacter throughout the sediment. Methanomicrobiales were the major competitors for hydrogen in high and low-sulfate environments. In the presence of sulfate, Methanosarcinaceae and Desulfobacteraceae became the most competitive acetate consumers, whereas Methanosaetaceae could outcompete these groups at low acetate and sulfate concentartions. On the other hand, mixed pure cultures of Methanosaeta concilii with hydrogenotrophic D. vulgaris and M. maripaludis indicated hydrogen leakage from M. concilii, which allowed D. vulgaris to grow and M. maripaludis to sustain itself. Hydrogen leakage provides an explanation for biogeochemical zonation for non-competitive and competitive substrates in marine sediments. This thesis provided insight into the relationship between sulfidogenic and methanogenic propionate and butyrate conversion at different depths of marine sediments, the role of $\mathrm{H}_{2}$ /formate and acetate during conversion and the responsible microbial community. It is apparent that several factors influence the interspecies interactions and conversion dynamics, such as temperature, sulfate availability, sulfide and methane concentrations, sediment depth and the syntrophic microbial community. Novel Syntrophomonas and Cryptanaerobacter species detected by $16 \mathrm{~S}$ rRNA gene sequencing analysis are likely involved in butyrate and propionate conversions, respectively, at different depths in marine sediments. Further attempts are necessary to isolate and characterize these microorganisms. As it is difficult to obtain syntrophs in pure or defined mixed cultures, different incubation conditions should be applied. These can include different substrates and substrate concentration, electron acceptor concentration, incubation temperatures and prolonged incubation time. The isolates can further be co-cultured with the organisms that are competitive or cooperative to get insight about interspecies interactions and their role in propionate and butyrate conversions in marine sediments.

While it is advantageous to monitor metabolic products and identify the metabolic pathways during incubation in batch systems, homogenization of sediment destroys the spatial structure of the microbial community. Continuous flow-through reactors can be used to cultivate microorganisms while maintaining low substrate concentrations comparable to in situ or to measure reaction rates without disrupting the porous structure in the sediment or spatial arrangements of microorganisms. However, long-term incubation is difficult with continuous flowthrough reactors as it is laborious to maintain the system steady for long periods. Intact sediment core incubation is another method that is used to mimic in situ conditions. Here, only the 
concentrations of carbon based substrates can be controlled while the pore-water constituents can be kept similar to in situ concentrations. Incubation with ${ }^{13} \mathrm{C}$-labelled substrates (e.g. acetate, propionate and butyrate) and subsequent ${ }^{13}$ C-labelled RNA/DNA/PLFA analysis can give information on the microorganisms using the labeled substrate, hence involving in the cycling of these substrates.

As the numerically rare community members may have important ecological and metabolic functions within marine sediments, the combination of single-cell methodologies with different FISH techniques can be applied for the analysis of less-abundant and more informative target microorganisms and their interactions within mixed populations. In this respect, after incubation with labeled substrates under in situ or near in situ conditions, subsamples can be chemically fixed for FISH and the cells can subsequently be visualized by nanoSIMS (Secondary Ion Mass Spectrometry). Simultaneous imaging of identity and the quantification of metabolic activity by nanoSIMS will provide insights into the physiology of microorganisms obtained both in axenic cultures and enrichments, as well as the ecophysiology in their natural environment. Proteins may also be considered as suitable targets for the direct identification of key enzymes involved in the uptake of particular labeled compounds. 

Appendices 



\section{References}

Achtnich C, Schuhmann A, Wind T, Conrad R (1995) Role of interspecies $\mathrm{H}_{2}$ transfer to sulfate and ferric iron-reducing bacteria in acetate consumption in anoxic paddy soil. FEMS Microbiol Ecol 16:61-69.

Ahlert S, Zimmermann R, Ebling J, König H (2016) Analysis of propionate-degrading consortia from agricultural biogas plants. Microbiologyopen 5: 1027-1037.

Albert DB, Martens CS (1997) Determination of low-molecular-weight organic acid concentrations in seawater and pore-water samples via HPLC. Mar Chem 56:27-37.

Alperin MJ, Reeburgh WS, Whitaker MJ (1988) Carbon and hydrogen isotope fractionation resulting from anaerobic methane oxidation. Glob Biochem Cycles 2: 279-288.

Alphenaar PA, Visser A, Lettinga G (1993) The effect of liquid upward velocity and hydraulic retention time on granulation in UASB reactors treating wastewater with a high sulphate content. Bioresource Technol 43(3):249-258.

Amann RI, Ludwig W, Schleifer KH, Amann RI, Ludwig W (1995) Phylogenetic identification and in situ detection of individual microbial cells without cultivation . Microbiol Rev 59:143-169.

Amend JP, Shock EL (2001) Energetics of overall metabolic reactions of thermophilic and hyperthermophilic Archaea and Bacteria. FEMS Microbiol Rev 25:175-243.

Aquilina A, Knab NJ, Knittel K, Kaur G, Geissler A, Kelly SP, Fossing H, Boot CS, Parkes RJ, Mills RA, Boetius A, Lloyd JR, Pancost RD (2010) Biomarker indicators for anaerobic oxidizers of methane in brackish-marine sediments with diffusive methane fluxes. Org Geochem 41:414426.

Bale NJ, Hopmans EC, Schoon PL, de Kluijver A, Downing JA, Middelburg JJ, Sinninghe Damsté JS, Schouten S (2016) Impact of trophic state on the distribution of intact polar lipids in surface waters of lakes. Limnol Oceanogr 61:1065-1077.

Banat IM, Nedwell DB (1983) Mechanisms of turnover of $\mathrm{C}_{2}-\mathrm{C}_{4}$ fatty acids in high-sulphate and low-sulphate anaerobic sediments. FEMS Microbiol Lett 17:107-110.

Barker HA (1936) On the biochemistry of the methane fermentation. Arch Mikrobiol 7:404-419.

Barnes RO, Goldberg ED (1976) Methane production and consumption in anoxic marine sediments. Geology 4:297-300.

Beaty PS, McInerney MJ (1987) Growth of Syntrophomonas wolfei in pure culture on crotonate. Arch Microbiol 147:389-393. 
Beck M, Riedel T, Graue J, Köster J, Kowalski N, Wu CS, Wegener G, Lipsewers Y, Freund H, Böttcher ME, Brumsack HJ, Cypionka H, Rullkötter J, Engelen B (2011) Imprint of past and present environmental conditions on microbiology and biogeochemistry of coastal Quaternary sediments. Biogeosciences 8:55-68.

Berry D, Mahfoudh K Ben, Wagner M, Loy A (2011) Barcoded primers used in multiplex amplicon pyrosequencing bias amplification. Appl Environ Microbiol 77:7846-7849.

Biddle JF, Fitz-Gibbon S, Schuster SC, Brenchley JE, House CH (2008) Metagenomic signatures of the Peru Margin subseafloor biosphere show a genetically distinct environment. Proc Natl Acad Sci 105:10583-10588.

Blair NE, Carter WD (1992) The carbon isotope biogeochemistry of acetate from a methanogenic marine sediment. Geochim Cosmochim Acta 56:1247-1258.

Bligh EG, Dyer WJ (1959) A rapid method of total lipid extraction and purification. Canad J of Biochem and Physiol 37:911-917.

Boetius A, Ravenschlag K, Schubert CJ, Rickert D, Widdel F, Gieseke A, Amann R, Jørgensen BB, Witte U, Pfannkuche $\mathrm{O}$ (2000) A marine microbial consortium apparently mediating anaerobic oxidation of methane. Nature 407:623-626.

Boone DR, Johnson RL, Liu Y (1989) Diffusion of the interspecies electron carriers $\mathrm{H}_{2}$ and formate in methanogenic ecosystems and its implications in the measurement of $\mathrm{Km} \mathrm{for} \mathrm{H}_{2}$ or formate uptake. Appl Environ Microbiol 55:1735-1741.

Boonet DR, Bryant MP (1980) Propionate-degrading bacterium, Syntrophobacter wolinii sp. nov. gen. nov., from methanogenic ecosystems. 40:626-632.

Borowski WS, Paull CK, Ussler W (1999) Global and local variations of interstitial sulphate gradients in the deep-water, continental margin sediments: Sensitivity to underlying methane and gas hydrates. Mar Geol 159:131-154.

Borrel G, Lehours AC, Crouzet O, Jézéquel D, Rockne K, Kulczak A, Duffaud E, Joblin K, Fonty G (2012) Stratification of Archaea in the deep sediments of a freshwater meromictic lake: vertical shift from methanogenic to uncultured Archaeal lineages. PLoS One. 7:e43346.

Bowles MW, Mogollon JM, Kasten S, Zabel M, Hinrichs K-U (2014) Global rates of marine sulfate reduction and implications for sub-sea-floor metabolic activities. Science 344:889-891.

Bragg L, Stone G, Imelfort M, Hugenholtz P, Tyson GW (2012) Fast, accurate error-correction of amplicon pyrosequences using Acacia. Nat Methods 9:425-426.

Burdige DJ (2007) Preservation of organic matter in marine sediments : controls, mechanisms, and an imbalance in sediment organic carbon budgets? Chem Rev 107:467-485. 
Callaghan AV, Morris BEL, Pereira IAC, McInerney MJ, Austin RN, Groves JT, Kukor JJ, Suflita JM, Young LY, Zylstra GJ, Wawrik B (2012) The genome sequence of Desulfatibacillum alkenivorans AK-o1: a blueprint for anaerobic alkane oxidation. Environ Microbiol 14:101-113.

Campbell BJ, Engel AS, Porter ML, Takai K (2006) The versatile $\epsilon$-proteobacteria: key players in sulphidic habitats. Nat Rev Microbiol 4:458-468.

Canfield DE (1993) Organic matter oxidation in marine sediments. In: Interactions of C, N, P, and S biogeochemical cycles and global change. Wollast, R, Chou, L, and Mackenzie, F, (eds) Springer-Verlag, Berlin Heidelberg pp 333-363.

Canfield D, Thamdrup B, Kristensen E (2005) Heterotrophic carbon metabolism. In: Aquatic Geomicrobiology. Southward, A., Tyler, P., Young, C., and Fuiman, L. (eds). Elsevier, Academic Press, pp 129-166.

Canfield DE, Thamdrup B, Kristensen E. (2006) Heterotrophic carbon metabolism. In: Aquatic geomicrobiology. Southward A. Tyler P, Young C, Fuiman, L, (eds) Elsevier, Academic Press, pp 129-166. F

Caporaso JG, Kuczynski J, Stombaugh J, Bittinger K, Bushman FD, Costello EK, Fierer N, Peña AG, Goodrich JK, Gordon JI, Huttley G, Kelley ST, Knights D, Koenig JE, Ley RE, Lozupone C, Mcdonald D, Muegge BD, Pirrung M, Reeder J, Sevinsky JR, Turnbaugh PJ, Walters W, Widmann J, Yatsunenko T, Zaneveld J, Knight R (2010) QIIME allows analysis of highthroughput community sequencing data. Nat Methods 7:335-336.

Cappenberg, TE (1974) Interrelations between sulfate-reducing and methane-producing bacteria in bottom deposits of a fresh-water lake. I. Field observations. Antonie Van Leeuwenhoek 40:285-295.

Cappenberg TE, Prins RA (1974) Interrelations between sulfate-reducing and methane-producing bacteria in bottom deposits of a fresh-water lake. III. Experiments with ${ }^{14} \mathrm{C}$-labeled substrates. Antonie Van Leeuwenhoek 40:457-469.

Chabrière E, Charon MH, Volbeda A, Pieulle L, Hatchikian EC, Fontecilla-Camps JC (1999) Crystal structures of the key anaerobic enzyme pyruvate:ferredoxin oxidoreductase, free and in complex with pyruvate. Nat Struct Biol 6:182-190.

Chapelle F, Lovley D. (1992) Competitive exclusion of sulfate reduction by Fe (III)-reducing bacteria: a mechanism for producing discrete zones of high-iron ground water. Ground Water 30: $29-36$.

Chen S, Liu X, Dong X (2005) Syntrophobacter sulfatireducens sp. nov., a novel syntrophic, propionate-oxidizing bacterium isolated from UASB reactors. Int J Syst Evol Microbiol 55:13191324.

Chong SC, Liu Y, Cummins M, Valentine DL, Boone DR (2002) Methanogenium marium sp. nov., a $\mathrm{H}_{2}$-using methanogen from Skan Bay, Alaska, and kinetics of $\mathrm{H}_{2}$ utilization. Antonie van Leeuwenhoek 81:263-270. 
Christensen D, Blackburn TH (1982) Turnover of ${ }^{14}$ C-labelled acetate in marine sediments. Mar Biol 71:113-119.

Christensen D (1984) Determination of substrates oxidized by sulfate reduction in intact cores of marine sediments. Limnol Oceanogr 29, 189-192.

Claypool GE, Kvenvolden KA (1983) Methane and other hydrocarbon gases in marine sediment. Annu Rev Earth Planet Sci 11:299-327.

Claypool GE (2004) Ventilation of marine sediments indicated by depth profiles of pore water sulfate and $\delta^{34}$ S. Geochemical Soc Spec Publ 9:59-65.

Claypool GE, Kvenvolden KA (1983) Methane and other hydrocarbon gases in marine sediment. Annu Rev Earth Pl Sc 11(1): 299-327.

Cline JJD (1969) Spectrophotometric determination of hydrogen sulfide in natural watersı. Limnol Oceanogr 14:454-458.

Conklin A, Stensel HD, Ferguson J (2006) Growth kinetics and competition between Methanosarcina and Methanosaeta in mesophilic anaerobic digestion. Water Environ Res 78:486-496.

Conrad R (1996) Soil microorganisms as controllers of atmospheric trace gases $\left(\mathrm{H}_{2}, \mathrm{CO}, \mathrm{CH}_{4}\right.$, OCS, $\mathrm{N}_{2} \mathrm{O}$, and NO). Microbiol Rev 6o:6o9-640.

Cord-Ruwisch R, Seitz HJ, Conrad R (1988) The capacity of hydrogenotrophic anaerobic bacteria to compete for traces of hydrogen depends on the redox potential of the terminal electron acceptor. Arch Microbiol 149:350-357.

Crill PM, Martens CS (1986) Methane production from bicarbonate and acetate in an anoxic marine sediment. Geochim. Cosmochim. Acta 50:2089-2097.

Daims H, Brühl A, Amann R, Schleifer K-H, Wagner M (1999) The domain-specific probe EUB338 is insufficient for the detection of all Bacteria: Development and evaluation of a more comprehensive probe set. Syst Appl Microbiol 22:434-444.

Dale AW, Aguilera DR, Regnier P, Fossing H, Knab NJ, Jørgensen BB (2008) Seasonal dynamics of the depth and rate of anaerobic oxidation of methane in Aarhus Bay (Denmark) sediments. J Mar Res 66:127-155.

Dar SA, Kleerebezem R, Stams AJM, Kuenen JG, Muyzer G (2008) Competition and coexistence of sulfate-reducing bacteria, acetogens and methanogens in a lab-scale anaerobic bioreactor as affected by changing substrate to sulfate ratio. Appl Microbiol Biotechnol 78:1045-1055.

Dar SA, Kuenen JG, Muyzer G (2005) Nested PCR-denaturing gradient gel electrophoresis approach to determine the diversity of sulfate-reducing bacteria in complex microbial communities. Appl Environ Microbiol 71:2325-2330. de Bok FAM, Stams AJM, Dijkema C, Boone DR (2001) 
Pathway of propionate oxidation by a syntrophic culture of Smithella propionica and Methanospirillum hungatei. Appl Environ Microbiol 67: 1800-1804.

de Bok FA, Roze EHA, Stams AJM (2002a) Hydrogenases and formate dehydrogenases of Syntrophobacter fumaroxidans. Antonie van Leeuwenhoek 81:283-291.

de Bok FAM, Luijten MLGC, Stams AJM (2002b) Biochemical evidence for formate transfer in syntrophic propionate-oxidizing cocultures of Syntrophobacter fumaroxidans and Methanospirillum hungatei. Appl Environ Microbiol 68:4247-4252.

de Bok FAM, Hagedoorn PL, Silva PJ, Hagen WR, Schiltz E, Fritsche K, Stams AJM (2003) Two Wcontaining formate dehydrogenases $\left(\mathrm{CO}_{2}\right.$-reductases) involved in syntrophic propionate oxidation by Syntrophobacter fumaroxidans. Eur J Biochem 270:2476-2485.

de Bok FAM, Plugge CM, Stams AJM (2004) Interspecies electron transfer in methanogenic propionate degrading consortia. Water Res 38:1368-1375.

de Bok FAM, Harmsen HJM, Plugge CM, de Vries MC, Akkermans ADL, de Vos WM, Stams AJM (2005) The first true obligately syntrophic propionate-oxidizing bacterium, Pelotomaculum schinkii sp. nov., co-cultured with Methanospirillum hungatei, and emended description of the genus Pelotomaculum. Int J Syst Evol Microbiol 55:1697-1703.

De Rosa M and Gambacorta A (1988) The lipids of archaebacteria. Prog Lipid Res 27:153-75.

Deegan LA, Johnson DS, Warren RS, Peterson BJ, Fleeger JW, Fagherazzi S, Wollheim WM (2012) Coastal eutrophication as a driver of salt marsh loss. Nature 490:388-392.

Delong EF (1992) Archaea in coastal marine environments. Proc Natl Acad Sci USA 89:5685-5689.

DeSantis TZ, Hugenholtz P, Keller K, Brodie EL, Larsen N, Piceno YM, Phan R, Andersen GL (20o6) NAST: a multiple sequence alignment server for comparative analysis of $16 \mathrm{~S}$ rRNA genes. Nucleic Acids Res 34:394-399.

Devereux R, Delaney M, Widdel F, Stahl DA (1989) Natural relationships among sulfate-reducing Eubacteria. J Bacteriol 171:6689-6695.

Dojka MA, Hugenholtz P, Haack SK, Pace NR (1998) Microbial diversity in a hydrocarbon-and chlorinated-solvent-contaminated aquifer undergoing intrinsic bioremediation. Appl Environ Microbiol 64:3869-3877.

Dolfing J, Jiang B, Henstra AM, Stams AJM, Plugge CM (2008) Syntrophic growth on formate: a new microbial niche in anoxic environments. Appl Environ Microbiol 74:6126-6131.

Dong X, Plugge CM, Stams AJM (1994) Anaerobic degradation of propionate by a mesophilic acetogenic bacterium in coculture and triculture with different methanogens. Appl Environ Microbiol 60:2834-2838. 
Dowhan W (1997) Molecular basis for membrane phospholipid diversity: why are there so many lipids? Ann Rev Biochem 66(1):199-232.

Edgar RC (2010) Search and clustering orders of magnitude faster than BLAST. Bioinformatics 26:2460-2461.

Ezaki T (2009) Peptococcaceae. In: Bergey's Manual of Systematic Bacteriology. De Vos P, Garrity G, Jones D, Krieg N, Ludwig W, Rainey F, Schleifer K.-H, Whitman W (eds). Springer-Verlag New York, USA pp 969-971.

Fang J, Barcelona MJ (1998) Structural determination and quantitative analysis of bacterial phospholipids using liquid chromatography/electrospray ionization/mass spectrometry. J Microbiol Methods 33:23-35.

Fang J, Barcelona MJ, Alvarez PJJ (2000) A direct comparison between fatty acid analysis and intact phospholipid profiling for microbial identification. Org Geochem 31:881-887.

Fang HHP, Liu Y, Ke SZ, Zhang T (2004) Anaerobic degradation of phenol in wastewater at ambient temperature. Water Sci Technol 49:95-102.

Fasham MJR, Balino BM, Bowles MC, Anderson R, Archer D, Bathmann U, Boyd P, Buesseler K, Burkil P, Bychkov A, Carlson C, Chen CTA, Doney S, Ducklow H, Emerson S, Feely R, Feldman G, Garcon V, Hansell D, Hanson R, Harrison P, Honjo S, Jeandel C, Karl D, Le Borgne R, Liu KK, Lochte K, Louanchi F, Lowry R, Michaels A, Monfray P, Murray J, Oschlies A, Platt T, Priddle J, Quinones R, Ruiz-Pino D, Saino T, Sakshaug E, Shimmield G, Smith S, Smith W, Takahashi T, Treguer P, Wallace D, Wanninkhof R, Watson A, Willebrand J, Wong CS (2001) A new vision of ocean biogeochemistry after a decade of the Joint Global Ocean Flux Study (JGOFS). Ambio 10: 4-31.

Finke N, Vandieken V, Jørgensen BB (2007a) Acetate, lactate, propionate, and isobutyrate as electron donors for iron and sulfate reduction in Arctic marine sediments, Svalbard. FEMS Microbiol Ecol 59:10-22.

Finke N, Hoehler TM, Jørgensen BB (2007b) Hydrogen 'leakage' during methanogenesis from methanol and methylamine: Implications for anaerobic carbon degradation pathways in aquatic sediments. Environ Microbiol 9:106o-1071.

Fry JC, Parkes RJ, Cragg BA, Weightman AJ, Webster G (2008) Prokaryotic biodiversity and activity in the deep subseafloor biosphere. FEMS Microbiol Ecol 66:181-196.

Garcia J-L, Ollivier B, Whitman WB (2006) The Order Methanomicrobiales. In: The Prokaryotes. Dworkin M, Falkow S, Rosenberg E, Schleifer K-H, Stackebrandt H (eds) Springer-Verlag, New York pp 208-230.

Glud RN, Gundersen JK, Røy H, Jørgensen BB (2003) Seasonal dynamics of benthic $\mathrm{O}_{2}$ uptake in a semienclosed bay: Importance of diffusion and faunal activity. Limnol Oceanogr 48:1265-1276. 
Godon JJ, Morinière J, Moletta M, Gaillac M, Bru V, Delgènes JP (2005) Rarity associated with specific ecological niches in the bacterial world: the "Synergistes" example. Environ Microbiol 7:213-224.

Greuter D, Loy A, Horn M, Rattei T (2016) ProbeBase-an online resource for rRNA-targeted oligonucleotide probes and primers: new features 2016. Nucleic Acids Res 44:586-589.

Grotenhuis JTC, Smit M, Plugge CM, Xu YS, van Lammeren AA, Stams AJM, Zehnder AJ (1991) Bacteriological composition and structure of granular sludge adapted to different substrates. Appl Environ Microbiol 57:1942-1949.

Gustafson WG, Feinberg BA, McFarland JT (1986) Energetics of beta-oxidation. Reduction potentials of general fatty acyl-CoA dehydrogenase, electron transfer flavoprotein, and fatty acyl-CoA substrates. J Biol Chem 261:7733-7741.

Halpern BS, Frazier M, Potapenko J, Casey KS, Koenig K, Longo C, Lowndes JS, Rockwood RC, Selig ER, Selkoe KA, Walbridge S (2015) Spatial and temporal changes in cumulative human impacts on the world's ocean. Nat Commun 6:7615.

Hamady M, Walker JJ, Harris JK, Gold NJ, Knight R (2008) Error-correcting barcoded primers for pyrosequencing hundreds of samples in multiplex. Nat Methods 5(3): 235-237.

Harmsen HJM, van Kuijk BLM, Plugge CM, Akkermans ADL, de Vos WM, Stams AJM (1998) Syntrophobacter fumaroxidans sp. nov., a syntrophic propionate-degrading sulfate-reducing bacterium Int J Syst Bacteriol 1383-1388.

Harrison BK, Zhang H, Berelson W, Orphan VJ (2009) Variations in archaeal and bacterial diversity associated with the sulfate-methane transition zone in continental margin sediments (Santa Barbara Basin, California). Appl Environ Microbiol 75:1487-1499.

Harvey HR, Fallon RD, Patton JS (1986) The effect of organic matter and oxygen on the degradation of bacterial membrane lipids in marine sediments. Geochim Cosmochim Acta 50:795-804.

Harvey HR (2006) Sources and cycling of organic matter in the marine water column. In: Marine Organic Matter: Biomarkers, Isotopes and DNA. Volkman JK (ed) Springer Heidelberg, Berlin, pp 1-25.

Hatamoto M, Imachi H, Fukayo S, Ohashi A, Harada H (2007) Syntrophomonas palmitatica sp. nov., an anaerobic syntrophic, long-chain fatty-acid-oxidizing bacterium isolated from methanogenic sludge. Int J Syst Evol Microbiol 57:2137-2142.

Hattori S, Kamagata Y, Hanada S, Shoun H (2000) Thermacetogenium phaeum gen. nov., sp. nov., a strictly anaerobic, thermophilic, syntrophic acetate-oxidizing bacterium. Int J Syst Evol Micr 5o(4): 1601-1609.

Hedges JI, Keil RG (1995) Sedimentary organic matter preservation: an assessment and speculative synthesis. Mar Chem 49:81-115. 
Henrichs SM (1992) Early diagenesis of organic matter in marine sediments: progress and perplexity. Mar Chem 39:119-149.

Herrmann S, Kleinsteuber S, Chatzinotas A, Kuppardt S, Lueders T, Richnow HH, Vogt C (2010) Functional characterization of an anaerobic benzene-degrading enrichment culture by DNA stable isotope probing. Environ Microbiol 12:401-411.

Hinrichs KU, Hayes JM, Sylva SP, Brewer PG, DeLong EF (1999) Methane-consuming archaebacteria in marine sediments. Nature 398:802-805.

Hoefs M, Schouten S, De Leeuw JW, King LL, Wakeham SG, Sinninghe Damsté JS (1997) Ether lipids of planktonic archaea in the marine water column. Appl Environ Microbiol 63:3090-3095.

Hoehler T, Alperin M, Albert D, Martens C (1998) Thermodynamic control on hydrogen concentrations in anoxic sediments. Geochim Cosmochim Acta 62: 1745-1756.

Hoehler TM, Alperin MJ, Albert DB, Martens CS (2001) Apparent minimum free energy requirements for methanogenic Archaea and sulfate-reducing bacteria in an anoxic marine sediment. FEMS Microbiol Ecol 38:33-41.

Holmer M, Kristensen E (1994) Coexistence of sulfate reduction and methane production in an organic-rich sediment. Mar Ecol Prog Ser 107:177-184.

Holmkvist L, Kamyshny A, Vogt C, Vamvakopoulos K, Ferdelman TG, Jørgensen BB (2011) Sulfate reduction below the sulfate-methane transition in Black Sea sediments. Deep Sea Res Part I Oceanogr Res Pap 58:493-504.

Hopmans EC, Schouten S, Pancost RD, van der Meer MTJ, Sinninghe Damsté JS (2000) Analysis of intact tetraether lipids in archaeal cell material and sediments by high performance liquid chromatography/atmospheric pressure chemical ionization mass spectrometry. Rapid Commun Mass Spectrom 14:585-589.

Imachi H, Sakai S, Ohashi A, Harada H, Hanada S, Kamagata Y, Sekiguchi Y (2007) Pelotomaculum propionicicum sp. nov., an anaerobic, mesophilic, obligately syntrophic, propionate-oxidizing bacterium. Int J Syst Evol Microbiol 57:1487-1492.

Imachi H, Sekiguchi Y, Kamagata Y, Hanada S, Ohashi A, Harada H (2002) Pelotomaculum thermopropionicum gen. nov., an anaerobic, thermophilic, syntrophic propionate-oxidizing bacterium. Int J Syst Evol Microbiol 52: 1729-1735.

Iversen N, Jorgensen BB (1985) Anaerobic methane oxidation rates at the sulfate-methane transition in marine sediments from Kattegat and Skagerrak (Denmark). Limnol Oceanogr 30:944-955.

Iwamoto TK, Tani K, Nakamura K, Suzuki Y, Kitagawa M, Eguchi M, Nasu M (2000) Monitoring impact of in situ biostimulation treatment on groundwater bacterial community by DGGE. FEMS Microbiol Ecol 32:129-141. 
Jackson BE, Bhupathiraju VK, Tanner RS, Woese CR, McInerney MJ (1999) Syntrophus aciditrophicus sp. nov., a new anaerobic bacterium that degrades fatty acids and benzoate in syntrophic association with hydrogen- using microorganisms. Arch Microbiol 171:107-114.

Jetten MSM, Stams AJM, Zehnder AJB (1992) Methanogenesis from acetate: a comparison of the acetate metabolism in Methanothrix soehngenii and Methanosarcina spp. FEMS Microbiol Lett 88:181-197.

Jones WJ, Paynter M, Gupta R (1983) Characterization of Methanococcus maripaludis sp. nov., a new methanogen isolated from salt marsh sediment. Arch Microbiol 135:91-97.

Jørgensen BB (1978) A comparison of methods for the quantification of bacterial sulfate reduction in coastal marine sediments. I. Measurement with radiotracer techniques. Geomicrobiol J 1: 1128.

Jørgensen BB (1982) Mineralization of organic matter in the sea bed - the role of sulphate reduction. Nature 296:643-645.

Jørgensen BB (1983) Processes at the sediment water interface. In: The major biogeochemical cycles and their interactions. Bolin B, Cook R (eds.) pp 477-515.

Jørgensen BB, Bak F (1991) Pathways and microbiology of thiosulphate transformations and sulfate reduction in a marine sediment (Kattegat, Denmark). Appl Environ Microbiol 57:847-856.

Jørgensen BB (2006) Bacteria and marine biogeochemistry. In: Marine Geochemistry. Schulz H, Zabel M (eds) (Vol. 2) Springer, Berlin pp 169-206.

Jørgensen BB, Kasten S (2006) Sulfur cycling and methane oxidation. In: Marine Geochemistry. Springer Heidelberg, Berlin pp 271-309.

Jørgensen BB, Boetius A (2007) Feast and famine - microbial life in the deep-sea bed. Nat Rev Microbiol 5:770-781.

Jørgensen BB, Parkes RJ (2010) Role of sulfate reduction and methane production by organic carbon degradation in eutrophic fjord sediments (Limfjorden, Denmark). Limnol Oceanogr 55:13381352.

Jørgensen BB, Marshall IPG (2016) Slow microbial life in the seabed. Ann Rev Mar Sci 8:311-332.

Jung YT, Lee JS, Yoon JH (2016) Gaetbulibacter aquiaggeris sp nov., a member of the Flavobacteriaceae isolated from seawater. Int J Syst Evol Micr 66:1131-1137.

Juretschko S, Timmermann G, Schmid M, Schleifer KH, Pommerening-Röser A, Koops HP, Wagner M (1998) Combined molecular and conventional analyses of nitrifying bacterium diversity in activated sludge: Nitrosococcus mobilis and Nitrospira-like bacteria as dominant populations. Appl Environ Microbiol 64:3042-3051. 
Juteau P, Côté V, Duckett MF, Beaudet R, Lépine F, Villemur R, Bisaillon JG (2005) Cryptanaerobacter phenolicus gen. nov., sp. nov., an anaerobe that transforms phenol into benzoate via 4-hydroxybenzoate. Int J Syst Evol Microbiol 55:245-250.

Kate M (1993) Membrane lipids of Archaea. In: The Biochemistry of Archaea (Archaeabacteria). Kates M, Kushner DJ, Matheson AT (eds) Elsevier Science, Amsterdam pp 261-295.

Kato S, Kosaka T, Watanabe K (2009) Substrate-dependent transcriptomic shifts in Pelotomaculum thermopropionicum grown in syntrophic co-culture with Methanothermobacter thermautotrophicus. Microb Biotechnol 2:575-584.

Kendall MM, Liu Y, Boone DR (2006) Butyrate- and propionate-degrading syntrophs from permanently cold marine sediments in Skan Bay, Alaska, and description of Algorimarina butyrica gen. nov., sp. nov. FEMS Microbiol Lett 262:107-114.

Kendall MM, Boone DR (2006a) Cultivation of methanogens from shallow marine sediments at Hydrate Ridge, Oregon. Archaea 2:31-38.

Kendall MM, Boone DR (2006b) The Order Methanosarcinales. In: The Prokaryotes. Dworkin M, Falkow S, Rosenberg E, Schleifer K-H, Stackebrandt H (eds) Springer-Verlag, New York pp 244-256.

Kiene RP, Oremland RS, Catena A, Miller LG, Capone DG (1986) Metabolism of reduced methylated sulfur compounds in anaerobic sediments and by a pure culture of an estuarine methanogen. Appl Environ Microbiol 52:1037-1045.

Kim M, Oh HS, Park SC, Chun J (2014) Towards a taxonomic coherence between average nucleotide identity and $16 \mathrm{~S}$ rRNA gene sequence similarity for species demarcation of prokaryotes. Int J Syst Evol Microbiol 64:346-351.

King GM, Klug MJ, Lovley DR (1983) Metabolism of acetate, methanol, and methylated amines in intertidal sediments of Lowes Cove, Maine. Appl Environ Microbiol 45:1848-1853.

Kleikemper J, Schroth MH, Sigler W V., Schmucki M, Bernasconi SM, Zeyer J (2002) Activity and diversity of sulfate-reducing bacteria in a petroleum hydrocarbon-contaminated aquifer. Appl Environ Microbiol 68:1516-1523.

Kleinsteuber S, Schleinitz KM, Breitfeld J, Harms H, Richnow HH, Vogt C (2008) Molecular characterization of bacterial communities mineralizing benzene under sulfate-reducing conditions. FEMS Microbiol Ecol 66:143-157.

Kleinsteuber S, Schleinitz KM, Vogt C (2012) Key players and team play: anaerobic microbial communities in hydrocarbon-contaminated aquifers. Appl Microbiol Biotechnol 94:851-873.

Knittel K, Boetius A (2009) Anaerobic oxidation of methane: progress with an unknown process. Annu Rev Microbiol 63:311-334. 
Knittel K, Boetius A, Lemke A, Eilers H, Lochte K, Pfannkuche O, Linke P, Amann R (2003) Activity, distribution, and diversity of sulfate reducers and other bacteria in sediments above gas hydrate (Cascadia Margin, Oregon). Geomicrobiol J 20:269-294.

Koga Y, Nishihara M, Morii H, Akagawa-Matsushita M (1993) Ether polar lipids of methanogenic bacteria: structures, comparative aspects, and biosyntheses. Microbiol Rev 57:164-182.

Koizumi Y, Kojima H, Fukui M (2005) Potential sulfur metabolisms and associated bacteria within anoxic surface sediment from saline meromictic Lake Kaiike (Japan). FEMS Microbiol Ecol 52:297-305.

Kristensen E, Holmer M (2001) Decomposition of plant materials in marine sediment exposed to different electron acceptors $\left(\mathrm{O}_{2}, \mathrm{NO}_{3}{ }^{-}\right.$and $\left.\mathrm{SO}_{4}{ }^{2-}\right)$, with emphasis on substrate origin, degradation kinetics, and the role of bioturbation. Geochim Cosmochim Acta 65:419-433.

Kristjansson JK, Schönheit P, Thauer RK (1982) Different Ks values for hydrogen of methanogenic bacteria and sulfate reducing bacteria: an explanation for the apparent inhibition of methanogenesis by sulfate. Arch Microbiol 131: 278-282.

Krylova NI, Conrad R (1998) Thermodynamics of propionate degradation in methanogenic paddy soil. FEMS Microbiol Ecol 26:281-288.

Kuever J (2014a) The family Desulfovibrionaceae. In: The Prokaryotes. Rosenberg E, DeLong EF, Lory S, Stackebrandt E, Thompson F (eds). Springer Berlin-Heidelberg Verlag, Berlin pp 107133

Kuever J (2014b) The family Desulfobacteraceae. In: The Prokaryotes. Rosenberg E, DeLong EF, Lory S, Stackebrandt E, Thompson F (eds). Springer Berlin-Heidelberg Verlag, Berlin pp 45-73.

Kuever, J. (2014c) The Family Desulfobulbaceae. In: The Prokaryotes. Rosenberg E, DeLong EF, Lory S, Stackebrandt E, Thompson F (eds). Springer Berlin-Heidelberg Verlag, Berlin pp 75-86.

Kvist T, Ahring BK, Westermann P (2007) Archaeal diversity in Icelandic hot springs. FEMS Microbiol Ecol 59:71-80.

Laanbroek HJ, Pfennig N (1981) Oxidation of short-chain fatty acids by sulfate-reducing bacteria in freshwater and in marine sediments. Arch Microbiol 128(3): 330-335.

Lagesen K, Hallin P, Rødland EA, Stærfeldt HH, Rognes T, Ussery DW (2007) RNAmmer: consistent and rapid annotation of ribosomal RNA genes. Nucleic Acids Res 35:3100-3108.

Lane DJ (1991) 16S/23S rRNA sequencing. In: Nucleic acid techniques in bacterial systematics. Stackebrandt E, Goodfellow M (eds). Wiley \& Sons, Chichester, United Kingdom pp 115-175.

Leloup J, Fossing H, Kohls K, Holmkvist L, Borowski C, Jørgensen BB (2009) Sulfate-reducing bacteria in marine sediment (Aarhus Bay, Denmark): abundance and diversity related to geochemical zonation. Environ Microbiol 11:1278-1291. 
Leloup J, Loy A, Knab NJ, Borowski C, Wagner M, Jørgensen BB (2007) Diversity and abundance of sulfate-reducing microorganisms in the sulfate and methane zones of a marine sediment, Black Sea. Environ Microbiol 9:131-142.

Lever MA (2012) Acetogenesis in the energy-starved deep biosphere-a paradox? Front Microbiol 2:118.

Li F, Hinderberger J, Seedorf H, Zhang J, Buckel W, Thauer RK (2008) Coupled ferredoxin and crotonyl coenzyme A (CoA) reduction with NADH catalyzed by the butyryl-CoA dehydrogenase/Etf complex from Clostridium kluyveri. J Bacteriol 190:843-850.

Lipp JS, Morono Y, Inagaki F, Hinrichs K-U (2008) Significant contribution of Archaea to extant biomass in marine subsurface sediments. Nature 454:991-994.

Liu C, Zhang XY, Wen XR, Shi M, Chen XL, Su HN (2016) Arcticiflavibacter luteus gen. nov., nov., sp. nov., a member of the family Flavobacteriaceae isolated from intertidal sand. Int J Syst Evol Microbiol 66:144-149.

Liu Q, Li J, Wei B, Zhang X, Zhang L, Zhang Y, Fang J (2016) Leeuwenhoekiella nanhaiensis sp. nov., isolated from deep-sea water. Int J Syst Evol Microbiol 66:1352-1357.

Liu Y, Balkwill DL, Aldrich HC, Drake GR, Boone DR (1999) Characterization of the anaerobic propionate- degrading syntrophs Smithella propionica gen. nov., sp. nov. and Syntrophobacter wolinii. Int J Syst Bacteriol 49:545-556.

Liu Y, Whitman WB (2008) Metabolic, phylogenetic, and ecological diversity of the methanogenic archaea. Ann N Y Acad Sci 1125:171-189.

Llobet-Brossa E, Rabus R, Böttcher ME, Könneke M, Finke N, Schramm A, Meyer RL, Grötzschel S, Rosselló-Mora R, Amann R (2002) Community structure and activity of sulfate-reducing bacteria in an intertidal surface sediment: a multi-method approach. Aquat Microb Ecol 29:211-226.

Lloyd KG, Lapham L, Teske A (2006) An anaerobic methane-oxidizing community of ANME-1b archaea in hypersaline gulf of Mexico sediments. Appl Environ Microbiol 72:7218-7230.

Lloyd KG, Schreiber L, Petersen DG, Kjeldsen KU, Lever MA, Steen AD, Stepanauskas R, Richter M, Kleindienst S, Lenk S, Schramm A, Jørgensen BB (2013) Predominant archaea in marine sediments degrade detrital proteins. Nature 496:215-218.

Lohner ST, Deutzmann JS, Logan BE, Leigh J, Spormann AM (2014) Hydrogenase-independent uptake and metabolism of electrons by the archaeon Methanococcus maripaludis. ISME J 8:1673-1681.

Lorowitz WH, Zhao H, Bryant MP (1989) Syntrophomonas wolfei subsp. saponavida subsp. nov., a long-chain fatty-acid degrading, anaerobic, syntrophic bacterium; Syntrophomonas wolfei 
subsp. wolfei supsp. nov.; and emended descriptions of the genus and species. Int J Syst Bacteriol 39:122-126.

Lösekann T, Knittel K, Nadalig T, Fuchs B, Niemann H, Boetius A, Amann R (2007) Diversity and abundance of aerobic and anaerobic methane oxidizers at the Haakon Mosby Mud Volcano, Barents Sea. Appl Environ Microbiol 73:3348-3362.

Lovley DR, Ferry JG (1985) Production and consumption of hydrogen during growth of Methanosarcina spp. on acetate. Appl Environ Microbiol 49:247-249.

Lovley DR (1985) Minimum threshold for hydrogen metabolism in methanogenic bacteria. Appl Environ Microbiol 49:1530-1531.

Lovley DR, Dwyer DF, Klug MJ (1982) Kinetic analysis of competition between sulfate reducters and methanogens for hydrogen in sediments. Appl Environ Microbiol 43:1373-1379.

Lovley D, Goodwin S (1988) Hydrogen concentrations as an indicator of the predominant terminal electron-accepting reactions in aquatic sediments. Geochim Cosmochim Acta 52: 2993-3003.

Lyimo TJ, Pol A, Op den Camp HJM, Harhangi HR, Vogels GD (2000) Methanosarcina semesiae sp. nov., a dimethylsulfide-utilizing methanogen from mangrove sediment. Int J Syst Evol Microbiol 50:171-178.

Maltby J, Sommer S, Dale AW, Treude T (2016) Microbial methanogenesis in the sulfate-reducing zone of surface sediments traversing the Peruvian margin. Biogeosciences 13:283-299.

Martens CS, Berner RA (1977) Interstitial water chemistry of anoxic Long Island Sound sediments 1. Dissolved-gases. Limnol Oceanogr 22:10-25.

Martens CS, Berner RA (1974) Methane production in the interstitial waters of sulfate-depleted marine sediments. Science (80- ) 185:1167-1169.

McGarigal K, Cushman S, Stafford S (200o) Multivariate statistics for wildlife and ecology research. New York, New York, USA: Springer.

McInerney MJ, Bryant MP, Pfennig N (1979) Anaerobic bacterium that degrades fatty acids in syntrophic association with methanogens. Arch Microbiol 122:129-135.

McInerney MJ, Bryant MP, Hespell RB, Costerton JW (1981) Syntrophomonas wolfei gen. nov. sp. nov., an anaerobic, syntrophic, fatty acid-oxidizing bacterium. Appl Environ Microbiol 41:1029-39.

McInerney MJ, Bryant MP (1981) Anaerobic degradation of lactate by syntrophic associations of Methanosarcina barkeri and Desulfovibrio species and effect of $\mathrm{H}_{2}$ on acetate degradation. Appl Environ Microbiol 41:346-354. 
McInerney MJ, Beaty PS (1988) Anaerobic community structure from a nonequilibrium thermodynamic perspective. Can J Microbiol 34:487-493.

McInerney MJ, Stams AJM, Boone DR (2005) Genus Syntrophobacter. In: Bergey's Manual of Systematic Bacteriology, second edition, vol 2. Staley JT, Boone DR, Brenner DJ, de Vos P, Garrity GM, Goodfellow M, Krieg NR, Rainey FA, Schleifer KH (eds). Springer, New York pp 1021-1027.

McInerney MJ, Rohlin L, Mouttaki H, Kim U, Krupp RS, Rios-Hernandez L, Sieber J, Struchtemeyer CG, Bhattacharyya A, Campbell JW, Gunsalus RP (2007) The genome of Syntrophus aciditrophicus: Life at the thermodynamic limit of microbial growth. Proc Natl Acad Sci USA 104:7600-7605.

McInerney MJ, Struchtemeyer CG, Sieber J, Mouttaki H, Stams AJM, Schink B, Rholin L, Gunsalus RP (2008) Physiology, ecology, phylogeny, and genomics of microorganisms capable of syntrophic metabolism. Ann NY Acad Sci 1125: 58-72.

McInerney MJ, Sieber JR, Gunsalus RP (2009) Syntrophy in anaerobic global carbon cycles. Curr Opin Biotechnol 20: 623-32.

Millero FJ, Schreiber DR (1982) Use of the ion pairing model to estimate activity coefficients of the ionic components of natural waters. Am. J. Sci. 282:1508-1540.

Mino S, Kudo H, Arai T, Sawabe T, Takai K, Nakagawa S (2014) Sulfurovum aggregans sp. nov., a hydrogen-oxidizing, thiosulfate-reducing chemolithoautotroph within the Epsilonproteobacteria isolated from a deep-sea hydrothermal vent chimney, and an emended description of the genus Sulfurovum. Int J Syst Evol Microbiol 64:3195-3201.

Mitterer RM (2010) Methanogenesis and sulfate reduction in marine sediments: a new model. Earth Planet Sci Lett 295:358-366.

Moore EK, Hopmans EC, Rijpstra WIC, Villanueva L, Sinninghe Damsté JS (2016) Elucidation and identification of amino acid containing membrane lipids using liquid chromatography/highresolution mass spectrometry. Rapid Commun Mass Spectrom 30:739-750.

Moore EK, Hopmans EC, Rijpstra WIC, Sánchez-Andrea I, Villanueva L, Wienk H, Schoutsen F, Stams AJM, Sinninghe Damsté JS (2015a) Lysine and novel hydroxylysine lipids in soil bacteria: amino acid membrane lipid response to temperature and $\mathrm{pH}$ in Pseudopedobacter saltans. Front Microbiol 6:637.

Moore EK, Villanueva L, Hopmans EC, Rijpstra WIC, Mets A, Dedysh SN, Sinninghe Damsté JS (2015b) Abundant trimethylornithine lipids and specific gene sequences are indicative of Planctomycete importance at the oxic/anoxic interface in Sphagnum-dominated northern wetlands. Appl Environ Microbiol 81:6333-6344.

Moore EK, Hopmans EC, Rijpstra WIC, Villanueva L, Dedysh SN, Kulichevskaya IS, Wienk H, Schoutsen F, Sinninghe Damsté JS (2013) Novel mono-, di-, and trimethylornithine membrane lipids in northern wetland Planctomycetes. Appl Environ Microbiol 79:6874-6884. 
Mori K, Iino T, Suzuki KI, Yamaguchi K, Kamagata Y (2012) Aceticlastic and NaCl-requiring methanogen "Methanosaeta pelagica" sp. nov., isolated from marine tidal flat sediment. Appl Environ Microbiol 78:3416-3423.

Morris BEL, Henneberger R, Huber H, Moissl-Eichinger C (2013) Microbial syntrophy: interaction for the common good. FEMS Microbiol Rev 37:384-406.

Mountfort DO, Asher RA (1979) Effect of inorganic sulfide on the growth and metabolism of Methanosarcina barkeri strain DM. Appl Environ Microbiol 37:670-675.

Mountfort DO, Asher RA (1981) Role of sulfate reduction versus methanogenesis in terminal carbon flow in polluted intertidal sediment of Waimea Inlet, Nelson, New Zealand. Appl Environ Microbiol 42:252-258.

Müller N, Schleheck D, Schink B (2009) Involvement of NADH:acceptor oxidoreductase and butyryl coenzymeA dehydrogenase in reversed electron transport during syntrophic butyrate oxidation by Syntrophomonas wolfei. J Bacteriol 191:6167-6177.

Müller N, Worm P, Schink B, Stams AJM, Plugge CM (2010) Syntrophic butyrate and propionate oxidation processes: from genomes to reaction mechanisms. Environ Microbiol Rep 2:489499.

Murray JW, Grundmanis V, Smethie WM (1978) Interstitial water chemistry in the sediments of Saanich Inlet. Geochim Cosmochim Acta 42:1011-1026.

Muyzer G, de Waal EC, Uitterlinden AG (1993) Profiling of complex microbial populations by denaturing gradient gel electrophoresis analysis of polymerase chain reaction-amplified genes coding for 16S rRNA. Appl Environ Microbiol 59: 695-700.

Muyzer G, Stams AJM (2008) The ecology and biotechnology of sulphate-reducing bacteria. Nat Rev Microbiol 6:441-454.

Nauhaus K, Boetius a, Kruger M, Widdel F (2002) In vitro demonstration of anaerobic oxidation of methane coupled to sulphate reduction in sediment from a marine gas hydrate area. Environ Microbiol 4:296-305.

Nauhaus K, Treude T, Boetius A, Krüger M (2005) Environmental regulation of the anaerobic oxidation of methane: a comparison of ANME-I and ANME-II communities. Environ Microbiol 7:98-106.

Niemann H, Losekann T, de Beer D, Elvert M, Nadalig T, Knittel K, Amann R, Sauter EJ, Schluter M, Klages M, Foucher JP, Boetius A (2006) Novel microbial communities of the Haakon Mosby mud volcano and their role as a methane sink. Nature 443:854-858.

Nübel U, Engelen B, Felske A, Snaidr J, Wieshuber A, Amann RI, Ludwig W, Backhaus H (1996) Sequence heterogeneities of genes encoding $16 \mathrm{~S}$ rRNAs in Paenibacillus polymyxa detected by temperature gradient gel electrophoresis. J Bacteriol 178:5636-5643. 
O'Flaherty V, Mahony T, O'Kennedy R, Colleran E (1998) Effect of pH on growth kinetics and sulphide toxicity thresholds of a range of methanogenic, syntrophic and sulphate-reducing bacteria. Process Biochem 33:555-569.

O’Sullivan LA, Sass AM, Webster G, Fry JC, Parkes RJ, Weightman AJ (2013) Contrasting relationships between biogeochemistry and prokaryotic diversity depth profiles along an estuarine sediment gradient. FEMS Microbiol Ecol 85:143-157.

Oremland RS, Taylor BF (1978) Sulfate reduction and methanogenesis in marine sediments. Geochim Cosmochim Acta 42:209-214.

Oremland RS, Marsh L, DesMarais DJ (1982a) Methanogenesis in Big Soda Lake, Nevada: an alkaline, moderately hypersaline desert lake. Appl Environ Microbiol 43:462-468.

Oremland RS, Marsh LM, Polcin S (1982b) Methane production and simultaneous sulphate reduction in anoxic, salt marsh sediments. Nature 296:143-145.

Oremland RS, Polcin S (1982) Methanogenesis and sulfate reduction: competitive and noncompetitive substrates in estuarine sediments. Appl Environ Microbiol 44:1270-1276.

Oremland RS, Miller LG, Whiticar MJ (1987) Sources and flux of natural gasses from Mono Lake, California. Geochim Cosmochim Acta 51:2915-2929.

Oremland RS, Whiticar MJ, Strohmaier FE, Kiene RP (1988) Bacterial ethane formation from reduced, ethylated sulfur compounds in anoxic sediments. Geochim Cosmochim Acta 52:18951904 .

Oude Elferink SJWH, Visser A, Hulshoff Pol LW, Stams AJM (1994) Sulfate reduction in methanogenic bioreactors. FEMS Microbiol Rev 15:119-136.

Oude Elferink SJWH, Vorstman WJC, Sopjes A, Stams AJM (1998) Characterization of the sulfatereducing and syntrophic population in granular sludge from a full-scale anaerobic reactor treating papermill wastewater. FEMS Microbiol Ecol 27:185-194.

Ozuolmez D, Na H, Lever MA, Kjeldsen KU, Jørgensen BB, Plugge CM (2015) Methanogenic archaea and sulfate reducing bacteria co-cultured on acetate: teamwork or coexistence? Front Microbiol 6:1-12.

Park S, Kim S, Jung YT, Park JM, Yoon JH (2016) Confluentibacter lentus gen. nov., sp. nov., isolated from the junction between the ocean and a freshwater lake. Int J Syst Evol Microbiol 66:868873.

Parkes RJ, Gibson GR, Mueller-Harvey I, Buckingham WJ, Herbert RA (1989) Determination of the substrates for sulphate-reducing bacteria within marine and estuarine sediments with different rates of sulphate reduction. J Gen Microbiol 135:175-187. 
Parkes RJ, Cragg BA, Fry JC, Herbert RA, Wimpenny JWT, Allen JA, Whitfield M (1990) Bacterial biomass and activity in deep sediment layers from the Peru Margin. Philos Trans R Soc London Ser A, Math Phys Sci 331:139-153.

Parkes RJ, Webster G, Cragg BA, Weightman AJ, Newberry CJ, Ferdelman TG, Kallmeyer J, Jørgensen BB, Aiello IW, Fry JC (2005) Deep sub-seafloor prokaryotes stimulated at interfaces over geological time. Nature 436:390-394.

Parkes RJ, Cragg BA, Banning N, Brock F, Webster G, Fry JC, Hornibrook E, Pancost RD, Kelly S, Knab N, Jørgensen BB, Rinna J, Weightman AJ (2007) Biogeochemistry and biodiversity of methane cycling in subsurface marine sediments (Skagerrak, Denmark). Environ Microbiol 9:1146-1161.

Parkes RJ, Cragg B, Roussel E, Webster G, Weightman A, Sass H (2014) A review of prokaryotic populations and processes in sub-seafloor sediments, including biosphere:geosphere interactions. Mar Geol 352:409-425.

Pender S, Toomey M, Carton M, Eardly D, Patching JW, Colleran E, O'Flaherty V (2004) Long-term effects of operating temperature and sulphate addition on the methanogenic community structure of anaerobic hybrid reactors. Water Res 38:619-630.

Phelps TJ, Conrad R, Zeikus JG (1985) Sulfate-dependent interspecies $\mathrm{H}_{2}$ transfer between Methnosarcina barkeri and Desulfovibrio vulgaris during coculture metabolism of acetate or methanol. Appl Environ Microbiol 50:589-594.

Pidwirny M (2012) Carbon cycle. In: Encyclopedia of Earth. Gulledge J (ed) (Washington D.C., Environmental information coaliation, national council for science and the environment). http://editors.eol.org/eoearth/wiki/Carbon cycle

Plugge CM, Jiang B, de Bok FAM, Tsai C, Stams AJM (2009) Effect of tungsten and molybdenum on growth of a syntrophic coculture of Syntrophobacter fumaroxidans and Methanospirillum hungatei. Arch Microbiol 191:55-61.

Plugge CM, Zhang W, Scholten JCM, Stams AJM (2011) Metabolic flexibility of sulfate-reducing bacteria. Front Microbiol 2:81.

Plugge CM, Balk M, Stams AJM (2002) Desulfotomaculum thermobenzoicum subsp. thermosyntrophicum subsp. nov., a thermophilic, syntrophic, propionate-oxidizing, sporeforming bacterium. Int J Syst Evol Micr 52:391-399.

Pruesse E, Quast C, Knittel K, Fuchs BM, Ludwig W, Peplies J, Glöckner FO (2007) SILVA: a comprehensive online resource for quality checked and aligned ribosomal RNA sequence data compatible with ARB. Nucleic Acids Res 35:7188-7196.

Qiu YL, Sekiguchi Y, Hanada S, Imachi H, Tseng I-C, Cheng S-S, Ohashi A, Harada H, Kamagata Y (2006) Pelotomaculum terephthalicum sp. nov. and Pelotomaculum isophthalicum sp. nov.: 
two anaerobic bacteria that degrade phthalate isomers in syntrophic association with hydrogenotrophic methanogens. Arch Microbiol 185:172-182.

Quast C, Pruesse E, Yilmaz P, Gerken J, Schweer T, Yarza P, Peplies J, Glöckner FO (2013) The SILVA ribosomal RNA gene database project: improved data processing and web-based tools. Nucleic Acids Res 41:590-596.

Rabus R, Hansen TA Widdel F (2013) Dissimilatory sulfate- and sulfur-reducing prokaryotes. In: The Prokaryotes - Prokaryotic Physiology and Biochemistry. Rosenberg E, DeLong EF, Lory S, Stackebrandt E, Thompson F (eds). Springer-Verlag Heidelberg, Berlin pp 309-404.

Ramiro-Garcia J, Hermes GDA, Giatsis C, Sipkema D, Zoetendal EG, Schaap PJ, Smidt H (2016) NGTax, a highly accurate and validated pipeline for analysis of $16 \mathrm{~S}$ rRNA amplicons from complex biomes. F1oooResearch 5:1791.

Raskin L, Rittmann BE, Stahl DA (1996) Competition and coexistence of sulfate-reducing and methanogenic populations in anaerobic biofilms. Appl Environ Microbiol 62:3847-3857.

Rasmussen H, Jørgensen BB (1992) Microelectrode studies of seasonal oxygen uptake in a coastal sediment: Role of molecular diffusion. Mar Ecol Prog Ser 81:289-303.

Rebac S, Visser A, Gerbens S, van Lier JB, Stams AJM, Lettinga G (1996) The Effect of sulphate on propionate and butyrate degradation in a psychrophilic anaerobic expanded granular sludge bed (EGSB) reactor. Environ Technol 17:997-1005.

Reeburgh WS (1980) Anaerobic methane oxidation: rate depth distributions in Skan Bay sediments. Earth Planet Sci Lett 47:345-352.

Reeburgh, W.S., Heggie, D.T. (1977) Microbial methane consumption reactions and their effects on methane distributions on freshwater and marine environments. Limnol Oceanogr 22: 1-9.

Roest K, Heilig HGHJ, Smidt H, de Vos WM, Stams AJM, Akkermans ADL (2005) Community analysis of a full-scale anaerobic bioreactor treating paper mill wastewater. Syst Appl Microbiol 28:175-185.

Romesser JA, Wolfe RS, Mayer F, Spiess E, Walther-Mauruschat A (1979) Methanogenium, a new genus of marine methanogenic bacteria and characterization of Methanogenium cariaci sp. nov. and Methanogenium marisnigri sp. nov. Arch Microbiol 121:147-153.

Rossel PE, Elvert M, Ramette A, Boetius A, Hinrichs KU (2011) Factors controlling the distribution of anaerobic methanotrophic communities in marine environments: evidence from intact polar membrane lipids. Geochim Cosmochim Acta 75:164-184.

Rossel PE, Lipp JS, Fredricks HF, Arnds J, Boetius A, Elvert M, Hinrichs KU (2008) Intact polar lipids of anaerobic methanotrophic archaea and associated bacteria. Org Geochem 39:992-999. 
Rotaru A-E, Shrestha PM, Liu F, Shrestha M, Shrestha D, Embree M, Zengler K, Wardman C, Nevin KP, Lovley DR (2014) A new model for electron flow during anaerobic digestion: direct interspecies electron transfer to Methanosaeta for the reduction of carbon dioxide to methane. Energy Environ Sci 7:408-415.

Roussel EG, Cragg BA, Webster G, Sass H, Tang X, Williams AS, Gorra R, Weightman AJ, Parkes RJ (2015) Complex coupled metabolic and prokaryotic community responses to increasing temperatures in anaerobic marine sediments: critical temperatures and substrate changes. FEMS Microbiol Ecol 91:1-16.

Roussel EG, Sauvadet A-L, Allard J, Chaduteau C, Richard P, Bonavita M-AC, Chaumillon E (2009) Archaeal methane cycling communities associated with gassy subsurface sediments of Marennes-Oléron Bay (France). Geomicrobiol J 26:31-43.

Roy F, Samain E, Dubourguier HC, Albagnac G (1986) Synthrophomonas sapovorans sp. nov., a new obligately proton reducing anaerobe oxidizing saturated and unsaturated long chain fatty acids. Arch Microbiol 145:142-147.

Rütters H, Sass H, Cypionka H, Rullkötter J (2002a) Phospholipid analysis as a tool to study complex microbial communities in marine sediments. J Microbiol Methods 48:149-160.

Rütters H, Sass H, Cypionka H, Rullkötter J (2002b) Microbial communities in a Wadden Sea sediment core - clues from analyses of intact glyceride lipids, and related fatty acids. Org Geochem 33:803-816.

Sahm K, MacGregor BJ, Jørgensen BB, Stahl DA (1999) Sulphate reduction and vertical distribution of sulphate-reducing bacteria quantified by rRNA slot-blot hybridization in a coastal marine sediment. Environ Microbiol 1:65-74.

Sanguinetti CJ, Dias Neto E, Simpson AJG (1994) Rapid silver staining and recovery of PCR products separated on polyacrylamide gels. Biotechniques 17:915-919.

Schink B (1997) Energetics of syntrophic cooperation in methanogenic degradation. Microbiol Mol Biol Rev 61:262-280.

Schink B, Stams AJM (2013) Syntrophism among prokaryotes. In: The Prokaryotes. Rosenberg E, DeLong EF, Lory S, Stackebrandt E, Thompson F (eds) Springer Berlin-Heidelberg Verlag, Berlin pp 309-335.

Schippers A, Kock D, Höft C, Köweker G, Siegert M (2012) Quantification of microbial communities in subsurface marine sediments of the Black Sea and off Namibia. Front Microbiol 3:1-11.

Schirawski J, Unden G (1998) Menaquinone-dependent succinate dehydrogenase of bacteria catalyzes reversed electron transport driven by the proton potential. Eur J Biochem 257:210215 . 
Schnurer A, Schink B, Svensson BH (1996) Clostridium ultunense sp. nov., a mesophilic bacterium oxidizing acetate in syntrophic association with a hydrogenotrophic methanogenic bacterium. Int J Syst Bacteriol 46:1145-1152.

Schönheit P, Kristjansson JK, Thauer RK (1982) Kinetic mechanism for the ability of sulfate reducers to out-compete methanogens for acetate. Arch Microbiol 132:285-288.

Schouten S, Hopmans EC, Pancost RD, Sinninghe Damste JS (200o) Widespread occurrence of structurally diverse tetraether membrane lipids: Evidence for the ubiquitous presence of lowtemperature relatives of hyperthermophiles. Proc Natl Acad Sci 97:14421-14426.

Schubotz F, Wakeham SG, Lipp JS, Fredricks HF, Hinrichs KU (2009) Detection of microbial biomass by intact polar membrane lipid analysis in the water column and surface sediments of the Black Sea. Environ Microbiol 11:2720-2734.

Schubotz, F (2005) Investigation of intact polar lipids of bacteria isolated from deep marine subsurface (MSc Thesis. University of Bremen, Bremen, Germany).

Schut GJ, Adams MWW (2009) The iron-hydrogenase of Thermotoga maritima utilizes ferredoxin and NADH synergistically: a new perspective on anaerobic hydrogen production. J Bacteriol 191:4451-4457.

Sekiguchi Y, Kamagata Y, Nakamura K, Ohashi A, Harada H (2000) Syntrophothermus lipocalidus gen. nov., sp. nov., a novel thermophilic, syntrophic, fatty-acid-oxidizing anaerobe which utilizes isobutyrate. Int J Syst Evol Micr 50:771-779.

Senior E, Lindström EB, Banat IM, Nedwell DB (1982) Sulfate reduction and methanogenesis in the sediment of a saltmarsh on the East coast of the United Kingdom. Appl Environ Microbiol 43:987-996.

Shaw DG, McIntosh DJ (1990) Acetate in recent anoxic sediments: direct and indirect measurements of concentration and turnover rates. Estuar Coast Shelf Sci 31:775-788.

Shimada H, Nemoto N, Shida Y, Oshima T, Yamagishi A (2008) Effects of pH and temperature on the composition of polar lipids in Thermoplasma acidophilum HO-62. J Bacteriol 190:54045411.

Shimizu S, Ueno A, Naganuma T, Kaneko K (2015) Methanosarcina subterranea sp. nov., a methanogenic archaeon isolated from a deep subsurface diatomaceous shale formation. Int J Syst Evol Microbiol 65:1167-1171.

Shin HS, Jung JY, Bae BU, Paik BC (1995) Phase-separated anaerobic toxicity assays for sulfate and sulfide. Water Environ Res 67:802-806.

Shock EL, Helgeson HC (1990) Calculation of the thermodynamic and transport properties of aqueous species at high pressures and temperatures: standard partial molal properties of organic species. Geochim Cosmochim Acta 54:915-945. 
Shock EL, Sassani DC, Willis M, Sverjensky DA (1997) Inorganic species in geologic fluids: correlations among standard molal thermodynamic properties of aqueous ions and hydroxide complexes. Geochim Cosmochim Acta 61:907-950.

Sieber JR, Le HM, Mcinerney MJ (2014) The importance of hydrogen and formate transfer for syntrophic fatty, aromatic and alicyclic metabolism. Environ Microbiol 16:177-188.

Sieber JR, McInerney MJ, Gunsalus RP (2012) Genomic insights into syntrophy: the paradigm for anaerobic metabolic cooperation. Annu Rev Microbiol 66:429-452.

Sieber JR, Sims DR, Han C, Kim E, Lykidis A, Lapidus AL, McDonnald E, Rohlin L, Culley DE, Gunsalus R, McInerney MJ (2010) The genome of Syntrophomonas wolfei: new insights into syntrophic metabolism and biohydrogen production. Environ Microbiol 12:2289-2301.

Singh H, Du J, Ngo HTT, Won KH, Kim KY, Yi TH (2015) Pedobacter edaphicus sp. nov. isolated from forest soil in South Korea. Arch Microbiol 197:781-787.

Skillman LC, Evans PN, Naylor GE, Morvan B, Jarvis GN, Joblin KN (2004) 16S ribosomal DNAdirected PCR primers for ruminal methanogens and identification of methanogens colonising young lambs. Anaerobe 10:277-285.

Smith KS, Ingram-Smith C (2007) Methanosaeta, the forgotten methanogen? Trends Microbiol 15:150-155.

Sohlenkamp C, López-Lara IM, Geiger O (2003) Biosynthesis of phosphatidylcholine in bacteria. Prog Lipid Res 42:115-162.

Song J, Choi A, Im M, Joung Y, Yoshizawa S, Cho JC, Kogure K (2015) Aurantivirga profunda gen. nov., sp nov., isolated from deep-seawater, a novel member of the family Flavobacteriaceae. Int J Syst Evol Micr 65:144-149.

Sørensen J, Christensen D, Jørgensen BB (1981) Volatile fatty acids and hydrogen as substrates for sulfate-reducing bacteria in anaerobic marine sediment. Appl Environ Microbiol 42:5-11.

Sousa DZ, Smidt H, Alves MM, Stams AJM (2007a) Syntrophomonas zehnderi sp. nov., an anaerobe that degrades long-chain fatty acids in co-culture with Methanobacterium formicicum. Int J Syst Evol Microbiol 57:609-615.

Sousa DZ, Pereira MA, Smidt H, Stams AJM, Alves MM (2007b) Molecular assessment of complex microbial communities degrading long chain fatty acids in methanogenic bioreactors. FEMS Microbiol Ecol 6o:252-265.

Sousa DZ, Alves JI, Alves MM, Smidt H, Stams AJM (2009) Effect of sulfate on methanogenic communities that degrade unsaturated and saturated long-chain fatty acids (LCFA). Environ Microbiol 11:68-80. 
Stackebrandt E (2014) The emended family Peptococcaceae and description of the families Desulfitobacteriaceae, Desulfotomaculaceae, and Thermincolaceae. In: The Prokaryotes. Rosenberg E, DeLong EF, Lory S, Stackebrandt E, Thompson F (eds). Springer-Verlag Heidelberg, Berlin pp 285-290.

Stams AJM, Grolle KCF, Frijters CTM, Van Lier JB (1992) Enrichment of thermophilic propionateoxidizing bacteria in syntrophy with Methanobacterium thermoautotrophicum or Methanobacterium thermoformicicum. Appl Environ Microbiol 58:346-352.

Stams AJM, Van Dijk JB, Dijkema C, Plugge CM (1993) Growth of syntrophic propionate-oxidizing bacteria with fumarate in the absence of methanogenic bacteria. Appl Environ Microbiol 59:1114-1119.

Stams AJM (1994) Metabolic interactions between anaerobic bacteria in methanogenic environments. Antonie Van Leeuwenhoek 66:271-294.

Stams AJM, Oude Elferink SWJH, Westermann P (2003) Metabolic interactions between methanogenic consortia and anaerobic respiring bacteria. In: Biomethanation I. T Scheper (eds.) Springer Berlin Heidelberg pp 31-56.

Stams AJM, Plugge CM, de Bok FAM, van Houten BHGW, Lens P, Dijkman H, Weijma J (2005) Metabolic interactions in methanogenic and sulfate-reducing bioreactors. Water Sci Technol 52:13-20.

Stams AJM, Plugge CM (2009) Electron transfer in syntrophic communities of anaerobic bacteria and archaea. Nat Rev Microbiol 7:568-577.

Starke R, Keller A, Jehmlich N, Vogt C, Richnow HH, Kleinsteuber S, von Bergen M, Seifert J (2016) Pulsed ${ }^{13} \mathrm{C}_{2}$-acetate protein-SIP unveils Epsilonproteobacteria as dominant acetate utilizers in a sulfate-reducing microbial community mineralizing benzene. Microb Ecol 71:901-911.

Stieb M, Schink B (1985) Anaerobic oxidation of fatty acids by Clostridium bryantii sp. nov., a sporeforming, obligately syntrophic bacterium. Arch Microbiol 140:387-390.

Struchtemeyer CG, Elshahed MS, Duncan KE, McInerney MJ (2005) Evidence for aceticlastic methanogenesis in the presence of sulfate in a gas condensate-contaminated aquifer. Appl Environ Microbiol 71:5348-5353.

Struchtemeyer CG, Duncan KE, Mcinerney MJ (2011) Evidence for syntrophic butyrate metabolism under sulfate-reducing conditions in a hydrocarbon-contaminated aquifer. FEMS Microbiol Ecol 76:289-300.

Sturt HF, Summons RE, Smith K, Elvert M, Hinrichs K-U (2004) Intact polar membrane lipids in prokaryotes and sediments deciphered by high-performance liquid chromatography/electrospray ionization multistage mass spectrometry-new biomarkers for biogeochemistry and microbial ecology. Rapid Commun Mass Spectrom 18:617-628. 
Suess E (1980) Particulate organic carbon flux in the oceans-surface productivity and oxygen utilization. Nature 288:260-263.

Sultan N, Garziglia S, Ruffine L (2016) New insights into the transport processes controlling the sulfate-methane-transition-zone near methane vents. Sci Rep 6:26701.

Summers ZM, Fogarty HE, Leang C, Franks AE, Malvankar NS, Lovley DR (2010) Direct exchange of electrons within aggregates of an evolved syntrophic coculture of anaerobic bacteria. Science 330:1413-1415.

Svetlitshny V, Rainey F, Wiegel J (1996) Thermosyntropha lipolytica gen. nov., sp. nov., a lipolytic, anaerobic, alkalitolerant, thermophilic bacterium utilizing short-and long-chain fatty acids in syntrophic coculture with a methanogenic archaeum. Int J Syst Evol Micr 46(4): 1131-1137.

Syvitski JPM, Vorosmarty CJ, Kettner AJ, Green P (2005) Impact of humans on the flux of terrestrial sediment to the global coastal ocean. Science 308: 376-380.

Szewzyk R, Pfennig N (1987) Complete oxidation of catechol by the strictly anaerobic sulfatereducing Desulfobacterium catecholicum sp. nov. Arch Microbiol 147:163-168.

Takahashi T, Feely R a, Weiss RF, Wanninkhof RH, Chipman DW, Sutherland SC, Takahashi TT (1997) Global air-sea flux of $\mathrm{CO}_{2}$ : an estimate based on measurements of sea-air $\mathrm{pCO}_{2}$ difference. Proc Natl Acad Sci U S A 94:8292-8299.

Takai K, Horikoshi K (200o) Rapid detection and quantification of members of the archaeal community by quantitative PCR using fluorogenic probes. Appl Environ Microbiol 66:50665072 .

Talaue-McManus L (2010) Examining human impacts on global biogeochemical cycling via the coastal zone and ocean margins. In: Carbon and nutrient fluxes in continental margins: A global synthesis. Liu KK, Atkinson L, Quinones R, Talaue-McManus L (eds) Springer-Verlag Berlin Heidelberg pp 497-514.

Teske AP (2006) Microbial community composition in deep marine subsurface sediments of ODP Leg 201: sequencing surveys and cultivations. In: Proc Ocean Drill Prog, Sci Res. Jørgensen BB, D’Hondt SL, Miller DJ (eds). Ocean Drilling Program, College Station, TX. 201: 1-19.

Teske A, Sørensen KB (2008) Uncultured archaea in deep marine subsurface sediments: have we caught them all? ISME J 2:3-18.

Thamdrup B, Rossello-Mora R, Amann R (2000) Microbial manganese and sulfate reduction in Black Sea shelf sediments. Appl Environ Microbiol 66:2888-2897.

Thauer RK, Jungermann K, Decker K (1977) Energy conservation in chemotrophic anaerobic bacteria. Bacteriol Rev 41:100-180.

Thomsen T (2001) Biogeochemical and molecular signatures of anaerobic methane oxidation in a marine sediment. Appl Environ Microbiol 67:1646-1656. 
Thomsen TR, Finster K, Ramsing NB (2001) Biogeochemical and molecular signatures of anaerobic methane oxidation in a marine sediment. Appl Environ Microbiol 67(4): 1646-1656.

Timmers PHA, Widjaja-Greefkes HCA, Ramiro-Garcia J, Plugge CM, Stams AJM (2015) Growth and activity of ANME clades with different sulfate and sulfide concentrations in the presence of methane. Front Microbiol.

Timmers PH, Suarez-Zuluaga DA, van Rossem M, Diender M, Stams AJM, Plugge CM (2016) Anaerobic oxidation of methane associated with sulfate reduction in a natural freshwater gas source. ISME J 10:1400-1412.

Ulrich AC, Edwards EA (2003) Physiological and molecular characterization of anaerobic benzenedegrading mixed cultures. Environ Microbiol 5(2): 92-102.

Valentine DL, Blanton DC, Reeburgh WS (2000) Hydrogen production by methanogens under lowhydrogen conditions. Arch Microbiol 174:415-421.

van den Bogert B, de Vos WM, Zoetendal EG, Kleerebezem M (2011) Microarray analysis and barcoded pyrosequencing provide consistent microbial profiles depending on the source of human intestinal samples. Appl Environ Microbiol 77:2071-2080.

van Kuijk BLM, Stams AJM (1996) Purification and characterization of malate dehydrogenase from the syntrophic propionate-oxidizing bacterium strain MPOB. FEMS Microbiol Lett 144:141144 .

van Kuijk BLM, Stams AJM (1995) Sulfate reduction by a syntrophic propionate-oxidizing bacterium. Antonie van Leeuwenhoek 68:293-296.

van Mooy BAS, Fredricks HF, Pedler BE, Dyhrman ST, Karl DM, Koblížek M, Lomas MW, Mincer TJ, Moore LR, Moutin T, Rappé MS, Webb EA (2009) Phytoplankton in the ocean use nonphosphorus lipids in response to phosphorus scarcity. Nature 458:69-72.

Vences-Guzmán MÁ, Guan Z, Ormeño-Orrillo E, González-Silva N, López-Lara IM, MartínezRomero E, Geiger O, Sohlenkamp C (2011) Hydroxylated ornithine lipids increase stress tolerance in Rhizobium tropici CIAT899. Mol Microbiol 79:1496-1514.

Viggi C, Rossetti S, Fazi S, Paiano P, Majone M, Aulenta F (2014) Magnetite particles triggering a faster and more robust syntrophic pathway of methanogenic propionate degradation. Environ Sci Technol 48(13): 7536-7543.

Vigneron A, Cruaud P, Pignet P, Caprais JC, Gayet N, Cambon-Bonavita MA, Godfroy A, Toffin L (2014) Bacterial communities and syntrophic associations involved in anaerobic oxidation of methane process of the sonora margin cold seeps, Guaymas basin. Environ Microbiol 16:27772790. 
Visscher PT, Baumgartner LK, Buckley DH, Rogers DR, Hogan ME, Raleigh CD, Turk KA, Des Marais DJ (2003) Dimethyl sulphide and methanethiol formation in microbial mats: potential pathways for biogenic signatures. Environ Microbiol 5:296-308.

Visser A, Beeksma I, van der Zee F, Stams AJM, Lettinga G (1993) Anaerobic degradation of volatile fatty acids at different sulphate concentrations. Appl Microbiol Biotechnol 40(4): 549-556.

Wagman DD, Evans WH, Parker VB, Schumm RH, Halow I, Bailey SM, Churney KL, Nuttall RL (1982) The NBS tables of chemical thermodynamic properties: selected values for inorganic and $C_{1}$ and $C_{2}$ organic substances in SI units. J. Phys. Chem. Ref. Data 11:392.

Wagner M, Loy A, Klein M, Lee N, Ramsing NB, Stahl DA, Friedrich MW (2005) Functional marker genes for identification of sulfate-reducing prokaryotes. Methods Enzymol 397:469-489.

Wallrabenstein C, Hauschild E, Schink B (1994) Pure culture and cytological properties of 'Syntrophobacter wolinii'. FEMS Microbiol Lett 123:249-254.

Wallrabenstein C, Hauschild E, Schink B (1995) Syntrophobacter pfennigii sp. nov., new syntrophically propionate-oxidizing anaerobe growing in pure culture with propionate and sulfate. Arch Microbiol 164:346-352.

Wang G, Spivack AJ, D'Hondt S (2010) Gibbs energies of reaction and microbial mutualism in anaerobic deep subseafloor sediments of ODP Site 1226. Geochim Cosmochim Acta 74:39383947.

Wang XC, Lee C (1995) Decomposition of aliphatic amines and amino acids in anoxic salt marsh sediment. Geochim Cosmochim Acta 59:1787-1797.

Wang Y, Wang H, Liu JW, Lai QL, Shao ZZ, Austin B, Zhang XH (2010) Aestuariibacter aggregatus sp. nov., a moderately halophilic bacterium isolated from seawater of the Yellow Sea. FEMS Microbiol Lett 309:48-54.

Wang Y, Zhou CY, Ming H, Kang J, Chen HL, Jing CQ, Feng H, Chang Y, Guo Z, Wang L (2016) Pseudofulvibacter marinus sp. nov., isolated from seawater. Int J Syst Evol Microbiol 66:13011305.

Wang Y, Qian PY (2009) Conservative fragments in bacterial 16S rRNA genes and primer design for 16S ribosomal DNA amplicons in metagenomic studies. PLoS One 4(10): p.e7401.

Ward DM, Winfrey MR (1985) Interactions between methanogenic and sulfate-reducing bacteria in sediments. In: Advances in microbial ecology. Jannasch HW and Williams PJL (eds.) Plenum Press, New York pp 219-286.

Webster G, Parkes RJ, Fry JC, Weightman J, Weightman AJ (2004) Widespread occurrence of a novel division of bacteria identified by $16 \mathrm{~S}$ rRNA gene sequences originally found in deep marine sediments. Appl Environ Microbiol 70:5708-5713. 
Webster G, Parkes RJ, Cragg BA, Newberry CJ, Weightman AJ, Fry JC (2006) Prokaryotic community composition and biogeochemical processes in deep subseafloor sediments from the Peru Margin. FEMS Microbiol Ecol 58(1): 65-85.

Webster G, Blazejak A, Cragg BA, Schippers A, Sass H, Rinna J, Tang X, Mathes F, Ferdelman TG, Fry JC, Weightman AJ, Parkes RJ (2009) Subsurface microbiology and biogeochemistry of a deep, cold-water carbonate mound from the Porcupine Seabight (IODP Expedition 307). Environ Microbiol 11:239-257.

Webster G, Rinna J, Roussel EG, Fry JC, Weightman AJ, Parkes RJ (2010) Prokaryotic functional diversity in different biogeochemical depth zones in tidal sediments of the Severn Estuary, UK, revealed by stable-isotope probing. FEMS Microbiol Ecol 72:179-197.

Webster G, Sass H, Cragg BA, Gorra R, Knab NJ, Green CJ, Mathes F, Fry JC, Weightman AJ, Parkes RJ (2011) Enrichment and cultivation of prokaryotes associated with the sulphate-methane transition zone of diffusion-controlled sediments of Aarhus Bay, Denmark, under heterotrophic conditions. FEMS Microbiol Ecol 77:248-263.

Wellsbury P, Mather I, Parkes RJ (2002) Geomicrobiology of deep, low organic carbon sediments in the Woodlark Basin, Pacific Ocean. FEMS Microbiol Ecol 42:59-70.

Wellsbury P, Parkes RJ (1995) Acetate bioavailability and turnover in an estuarine sediment. FEMS Microbiol Ecol 17:85-94.

Weng CY, Chen SC, Lai MC, Wu SY, Lin S, Yang TF, Chen PC (2015) Methanoculleus taiwanensis sp. nov., a methanogen isolated from deep marine sediment at the deformation front area near Taiwan. Int J Syst Evol Microbiol 65:1044-1049.

Weston NB, Joye SB (2005) Temperature-driven decoupling of key phases of organic matter degradation in marine sediments. Proc Natl Acad Sci U S A 102:17036-17040.

White DC, Davis WM, Nickels JS, King JD, Bobbie RJ (1979) Determination of the sedimentary microbial biomass by extractible lipid phosphate. Oecologia 40:51-62.

Whiticar MJ (1999) Carbon and hydrogen isotope systematics of bacterial formation and oxidation of methane. Chem Geol 161:291-314.

Widdel F (1980) Anaerober Abbau von Fettsäuren und Benzoesäure durch neu isolierte Arten sulfatreduziernder Bakterien. Thesis, Göttingen Univ.

Widdel F (1988) Microbiology and ecology of sulfate- and sulfur-reducing bacteria. In: Biology of Anaerobic Microorganisms. Zehnder AJB (ed). John Wiley and Sons Inc. New York pp 469585 .

Widdel F, Bak F (1992) Gram-negative mesophilic sulfate-reducing bacteria. In: The prokaryotes. A handbook on the biology of bacteria: ecophysiology, isolation, identification, applications. 2nd 
edn. Balows A, Truper HG, Dworkin M, Harder W, Schleifer K-H (eds) Springer Verlag, New York, USA pp 3353-3378.

Wilms R, Sass H, Köpke B, Cypionka H, Engelen B (2007) Methane and sulfate profiles within the subsurface of a tidal flat are reflected by the distribution of sulfate-reducing bacteria and methanogenic archaea. FEMS Microbiol Ecol 59:611-621.

Winderl C, Anneser B, Griebler C, Meckenstock RU, Lueders T (2008) Depth-resolved quantification of anaerobic toluene degraders and aquifer microbial community patterns in distinct redox zones of a tar oil contaminant plume. Appl Environ Microbiol 74:792-8o1.

Winfrey MR, Zeikus JG (1977) Effect of sulfate on carbon and electron flow during microbial methanogenesis in freshwater sediments. Appl Environ Microbiol 33:275-281.

Wofford NQ, Beaty PS, McInerney MJ (1986) Preparation of cell-free-extracts and the enzymes involved in fatty acid metabolism in Syntrophomonas wolfei. J Bacteriol 167:179-185.

Worm P, Stams AJM, Cheng X, Plugge CM (2011) Growth- and substrate-dependent transcription of formate dehydrogenase and hydrogenase coding genes in Syntrophobacter fumaroxidans and Methanospirillum hungatei. Microbiology 157:280-289.

Wu C, Dong X, Liu X (2007) Syntrophomonas wolfei subsp. methylbutyratica subsp. nov., and assignment of Syntrophomonas wolfei subsp. saponavida to Syntrophomonas saponavida sp. nov. comb. nov. Syst Appl Microbiol 30:376-380.

Wu C, Liu X, Dong X (2006a) Syntrophomonas cellicola sp. nov., a spore-forming syntrophic bacterium isolated from a distilled-spirit-fermenting cellar, and assignment of Syntrophospora bryantii to Syntrophomonas bryantii comb. nov. Int J Syst Evol Microbiol 56:2331-2335.

Wu C, Liu X, Dong X (2006b) Syntrophomonas erecta subsp. sporosyntropha subsp. nov., a sporeforming bacterium that degrades short chain fatty acids in co-culture with methanogens. Syst Appl Microbiol 29:457-462.

Yamada T, Sekiguchi Y (2009) Cultivation of uncultured Chloroflexi subphyla: significance and ecophysiology of formerly uncultured Chloroflexi "subphylum I" with natural and biotechnological relevance. Microbes Environ 24:205-216.

Yarza P, Yilmaz P, Pruesse E, Glöckner FO, Ludwig W, Schleifer K-H, Whitman WB, Euzéby J, Amann R, Rosselló-Móra R (2014) Uniting the classification of cultured and uncultured bacteria and archaea using 16S rRNA gene sequences. Nat Rev Microbiol 12:635-645.

Yim KJ, Cha I-T, Whon TW, Lee H-W, Song HS, Kim K-N, Nam Y-D, Lee S-J, Bae J-W, Rhee S-K, Choi J-S, Seo M-J, Roh SW, Kim D (2014) Halococcus sediminicola sp. nov., an extremely halophilic archaeon isolated from a marine sediment. Antonie Van Leeuwenhoek 105:73-79.

Yoon J, Kasai H (2016) Wenyingzhuangia aestuarii sp. nov., a marine bacterium of the family Flavobacteriaceae isolated from an estuary. Curr Microbiol 72:397-403. 
Yu Y, Lee C, Kim J, Hwang S (2005) Group-specific primer and probe sets to detect methanogenic communities using quantitative real-time polymerase chain reaction. Biotechnol Bioeng 89:670-679.

Yu ZT, García-González R, Schanbacher FL, Morrison M (2008) Evaluations of different hypervariable regions of archaeal $16 \mathrm{~S}$ rRNA genes in profiling of methanogens by Archaeaspecific PCR and denaturing gradient gel electrophoresis. Appl Environ Microbiol 74:889-893.

Zhang C, Liu X, Dong X (2004) Syntrophomonas curvata sp. nov., an anaerobe that degrades fatty acids in co-culture with methanogens. Int J Syst Evol Microbiol 54:969-973.

Zhang C, Liu X, Dong X (2005) Syntrophomonas erecta sp. nov., a novel anaerobe that syntrophically degrades short-chain fatty acids. Int J Syst Evol Microbiol 55:799-803.

Zhang T, Ke SZ, Liu Y, Fang HP (2005) Microbial characteristics of a methanogenic phenoldegrading sludge. Water Sci Technol 52:73-78.

Zhao H, Yang D, Woese CR, Bryant MP (1990) Assignment of Clostridium bryantii to Syntrophospora bryantii gen. nov., comb. nov. on the basis of a 16S rRNA sequence analysis of its crotonate-grown pure culture. Int J Syst Evol Micr 40:40-44.

Zhao H, Yang D, Woese CR, Bryant MP (1993) Assignment of fatty acid- $\beta$-oxidizing syntrophic bacteria to Syntrophomonadaceae fam. nov. on the basis of 165 rRNA sequence analyses. Int J Syst Bacteriol 43:278-286.

Zhao H, Yang D, Woese CR, Bryant MP (1990) Assignment of Clostridium bryantii to Syntrophospora bryantii gen. nov., com. nov. on the basis of a $16 \mathrm{~s}$ rRNA sequence analysis of its crotonate-grown pure culture. Int J Syst Bacteriol 40: 40-44.

Zinder SH, Koch M (1984) Non-aceticlastic methanogenesis from acetate: acetate oxidation by a thermophilic syntrophic coculture. Arch Microbiol 138:263-272. 


\section{Summary}

Propionate, butyrate, acetate, hydrogen and formate are the major intermediates of organic matter degradation. Sulfate-reducing bacteria (SRB) contribute significantly to the consumption of these substrates in sulfate-rich marine sediments. In sulfate-depleted sediments, however, complete degradation of propionate or butyrate is only possible via syntrophic cooperation of acetogenic bacteria and methanogenic archaea. Despite that the predominance of SRB in sulfate-rich and methanogens in sulfate-depleted sediments was reported, recent studies showed that both types of microorganism could be present in upper and lower parts of marine sediments. In this thesis, propionate and butyrate conversions and the involved microbial community in sulfate, sulfatemethane transition and methane zone sediment of Aarhus Bay, Denmark were studied using sediment slurry incubations. Interspecies hydrogen transfer and coexistence during acetate degradation were investigated in mixed pure cultures.

In Chapter 2, interspecies hydrogen transfer between aceticlastic Methanosaeta concilii and hydrogenotrophic microorganisms, Desulfovibrio vulgaris or Methanococcus maripaludis, was investigated. Additionally, coexistence of $M$. concilii and Desulfobacter latus growing on acetate under sulfidogenic conditions was studied. The results of Chapter 2 showed that D. vulgaris could reduce sulfate and grow on leaked hydrogen from M. concilii. Hydrogen leakage from M. concilii provides an explanation for biogeochemical zonation both for competitive (e.g. acetate) and noncompetitive substrates (methyl compounds), and this indicates the possible coexistence of SRB and methanogens in sulfate-rich environments.

In chapter 3 and 4 , long term incubations were examined focusing on butyrate and propionate conversion and the microbial community dynamics in sediment slurry enrichments at different sulfate $(0,3$ and $20 \mathrm{mM})$ concentrations and incubation temperatures $\left(10^{\circ} \mathrm{C}\right.$ and $\left.25^{\circ} \mathrm{C}\right)$. Sulfate reduction is the dominant process for butyrate and propionate conversion in Aarhus Bay sediments. In the absence of sulfate, both propionate and butyrate can be converted efficiently, indicating the presence of syntrophic communities throughout the sediment. The fluctuating methane concentrations and the enrichment of anaerobic methanotrophic archaea (ANME) in butyrate and propionate slurries at $10^{\circ} \mathrm{C}$ suggest the occurrence of anaerobic oxidation of methane $(\mathrm{AOM})$ in sulfate-methane transition zone (SMTZ) of Aarhus Bay.

The microbial community involved in butyrate and propionate conversion was investigated using next generation sequencing (NGS) 16S rRNA amplicon sequencing. The enriched sulfatereducing bacteria at high sulfate concentration $(20 \mathrm{mM})$ were different when butyrate and 
propionate were used as substrate: Desulfosarcina and Desulfobacterium dominate the butyrateconverting slurries (Chapter 3), whereas Desulfosarcina, Desulfobulbus and Desulforhopalus are the main SRB in propionate-converting slurries (Chapter 4). The increase in the relative abundance of Desulfobacteraceae and Desulfobulbaceae in SZ, SMTZ and MZ sediment slurries suggests the presence of sulfate reducers throughout the anoxic sediment column. In the absence of sulfate, Syntrophomonas and Cyrptanaerobacter become dominant which suggests their role in syntrophic butyrate and propionate conversion, respectively. These results were further supported in Chapter 6. The increase in the relative abundance of Syntrophomonas in the presence of sulfate (Chapter 3) and some members of Desulfobacteraceae (Chapter 4) in the absence of sulfate shows the metabolic flexibility of the microorganisms at different sulfate concentrations. Temperature has an impact on the microbial community (Chapter 4) and IPL composition (Chapter 5) in enrichment slurries. Cryptanaerobacter is dominant at $25^{\circ} \mathrm{C}$, and, Desulfobacteraceae (Desulfofaba), especially Desulfobulbaceae members (Desulfobulbus, Desulforhopalus) become dominant at $10^{\circ} \mathrm{C}$ at o and 3 $\mathrm{mM}$ sulfate concentrations in propionate-amended enrichment slurries. In butyrate-amended slurries, Clostridiales have higher relative abundance at $10^{\circ} \mathrm{C}$ regardless of the sulfate concentration and the sediment depth which supports important role of Clostridiales in butyrate conversion in marine sediments. Archaeal community analyses revealed the dominance of hydrogenotrophic methanogens belonging to Methanomicrobiales in both butyrate- and propionate-converting slurries (Chapter 3 and 4) and enrichment cultures (Chapter 6) regardless of the sediment depth, the incubation temperature and the presence of sulfate, which indicate that they are the main syntrophic partners of butyrate and propionate degraders. The other syntrophic partner organisms are the aceticlastic methanogenic families: Methanosarcinaceae and Methanosaetaeceae. The presence of methane-oxidizing archaea (ANME-1b) in low temperature SMTZ slurries together with Desulfobacteraceae (Chapter 3 and 4) suggests the occurrence of anaerobic oxidation of methane (AOM) in SMTZ of Aarhus Bay.

In conclusion, this thesis confirms the presence and activity of methanogens in sulfate-rich, and SRB in sulfate-depleted marine sediments and their involvement in butyrate, propionate and acetate conversion. Novel bacterial and archaeal members enriched in the sediment slurries are likely involved in propionate, butyrate and acetate conversions at different depths of marine sediments in addition to known the cultured species. 


\section{Samenvatting}

Propionaat, butyraat, acetaat, waterstof en formiaat zijn de voornaamste tussenproducten van afbraak van organisch materiaal. Sulfaat reducerende bacteriën (SRB) dragen significant bij aan de consumptie van deze substraten in sulfaat-rijke marine sedimenten. Echter, in sulfaat-arme sedimenten is complete afbraak van propionaat en butyraat alleen mogelijk via syntrofe interactie met acetogene bacteriën of methanogene archaea. Ondanks dat literatuur de dominantie van SRB in sulfaat-rijke, en methanogenen in sulfaat-arme sedimenten beschrijft, tonen recente studies aan dat beide typen micro-organismen aanwezig zijn in beide soorten sediment. In deze thesis worden propionaat en butyraat omzetting en de daarbij betrokken microbiële groepen in sulfaat-rijke zone (SZ), sulfaat-arme zone (MZ) en de sulfaat-methaan transitie zone (SMTZ) in het sediment van de Baai van Aarhus, Denemarken besproken aan de hand van sediment slurrie incubaties. Uitwisseling van waterstof en samenleving van micro-organismen gedurende acetaat afbraak zijn onderzocht gebruikmakende van gemixte pure cultures.

In hoofdstuk 2 wordt waterstof overdracht tussen de aceticlastische methanogeen Methanosaeta concilii en hydrotrofe microorganismen, Desulfovibrio vulgaris of Methanococcus maripaludis besproken. Ook de samenwerking tussen $M$. concilii en Desulfobacter latus groeiend op acetaat in sulfaat reducerende condities is bestudeerd. De resultaten van hoofdstuk 2 laten zien dat $D$. vulgaris sulfaat kan reduceren en groeien met waterstof gelekt uit $M$. concilii. Waterstof lekkage van $M$. concilii kan verklaren waarom er biogeochemisch een zonatie is van competitieve (b.v. acetaat) en niet-competitieve (b.v. gemethyleerde stoffen) substraten, en geeft indicatie dat SRB en methanogenen mogelijk samenleven in sulfaat-rijke omgeving.

In hoofdstuk 3 en 4 worden lange termijn incubaties besproken met focus op butyraat en propionaat omzetting, en de populatie dynamiek daarachter, bij sediment incubaties met verschillende sulfaat concentraties $(\mathrm{o}, 3$ en $20 \mathrm{mM})$ en incubatie temperatuur $\left(10{ }^{\circ} \mathrm{C}\right.$ en $\left.20{ }^{\circ} \mathrm{C}\right)$. Sulfaatreductie is het overheersende proces voor butyraat en propionaat omzetting in sedimenten van de Baai van Aarhus. In afwezigheid van sulfaat worden beide substraten efficiënt omgezet, wat aangeeft dat er syntrofe groepen aanwezig zijn in het sediment. Fluctuerende gehaltes van methaan en de verrijking van anaerobe methaan oxiderende archaea (ANME) gedurende butyraat en propionaat omzetting bij $10{ }^{\circ} \mathrm{C}$ suggereren dat er anaerobe oxidatie van methaan (AOM) plaatsvind in de sulfaat-methaan transitie zone van de Baai van Aarhus. 
De microben betrokken bij butyraat en propionaat omzetting zijn bestudeerd met next-generation sequencing (NGS) van het 16S rRNA amplicon. De verrijkte sulfaat-reducerende bacteriën bij hoge sulfaat concentraties $(20 \mathrm{mM})$ verschilde wanneer butyraat of propionaat werden gebruikt als substraat. Desulfosarcina en Desulfobacterium domineerde de butyraat-consumerende slurries (hoofdstuk 3), terwijl Desulfosarcina, Desulfobulbus en Desulforhopalus de voornaamste SRB zijn in propionaat-verbruikende slurries (Hoofdstuk 4). De toename in relatieve aanwezigheid van Desulfobacteraceae en Desulfobulbaceae in de SZ, SMTZ en MZ sediment incubaties suggereert de aanwezigheid van sulfaat reduceerders door de gehele anoxische sediment kolom. In de afwezigheid van sulfaat worden Synhrophomonas en Cyrptanaerobacter de dominante organismen, wat hun syntrofe rol in respectievelijk butyraat en propionaat omzetting suggereert. Deze resultaten zijn verder onderbouwt in Hoofdstuk 6. De toename in relatieve aanwezigheid van Syntrophomonas in de aanwezigheid van sulfaat (Hoofdstuk 3) en sommige leden van de Desulfobacteraceae (hoofdstuk 4) in afwezigheid van sulfaat laat de metabole flexibiliteit van micro-organismen bij verschillende sulfaat concentraties zien.

Temperatuur had effect op de samenstelling (hoofdstuk 4) en IPL compositie (hoofdstuk 5) van de microbiële groepen in de incubaties. Cryptanaerobacter is dominant bij 25 graden Celsius. Desulfobacteraceae (Desulfofaba), vooral Desulfobulbaceaea (Desulfobulbus, Desulforhopalus), worden dominant bij 10 graden Celsius bij o en $3 \mathrm{mM}$ sulfaat in propionaat-verrijkte slurries. In incubaties met butyraat zijn Clostridiales relatief meer aanwezig bij $10{ }^{\circ} \mathrm{C}$ ongeacht de sulfaatconcentratie en de sedimentdiepte, wat de belangrijke rol van Clostridiales in butyraat omzetting in marine sedimenten benadrukt. Analyse op de samenstelling van Archaea liet de dominatie zien van hydrogenotrofe methanogenen behorend tot de Methanomicrobiales in zowel butyraat en propionaat omzettende incubaties (hoofdstuk 3 en 4) als in verrijkte cultures (hoofdstuk 6) ongeacht sedimentdiepte, incubatietemperatuur en aanwezigheid van sulfaat. Dit geeft aan dat zij de voornaamste syntrofe partners zijn van butyraat en propionaat afbrekende micro-organismen. Andere syntrofe partner organismen zijn de aceticlastische methanogene families: Methanosarcinaceae en de Methanosaetaeceae. De aanwezigheid van methaan-oxiderende archaea (ANME-1b) bij lage temperatuur SMTZ incubaties, samen met de aanwezigheid van Desulfobacteraceae (hoofdstuk 3 en 4), suggereren dat anaerobe oxidatie van methaan in SMTZ van de Baai van Aarhus plaatsvind. 
Deze thesis bevestigt de aanwezigheid en activiteit van methanogenen in sulfaat-rijke, en SRB in de sulfaat-arme marine sedimenten; en hun betrokkenheid bij butyraat, propionaat en acetaat omzetting. Nieuwe bacteriële en archaea soorten zijn opgehoopt in de sediment slurrie incubaties en zijn waarschijnlijk betrokken bij propionaat, butyraat en acetaat omzetting in verschillende dieptes van het marine sedimenten en zijn een additie aan de al bekende soorten. 


\title{
Author affiliations
}

\author{
Alfons J. M. Stams, Caroline M. Plugge, M. Cristina Gagliano, Daan van Vliet, Derya \\ Ozuolmez \\ Laboratory of Microbiology, Wageningen University \& Research, Stippeneng 4, 6708 WE \\ Wageningen, The Netherlands.
}

\section{Alfons J. M. Stams}

Centre for Biological Engineering, University of Minho, Braga, Portugal

\section{Hyunsoo Na}

Division of Microbial Ecology, Department of Microbiology and Ecosystem Science, University of Vienna, Vienna, Austria.

\section{Mark A. Lever}

Institute of Biogeochemistry and Pollutant Dynamics, Department of Environmental Sciences, Eidgenössische Technische Hochschule Zurich, Zürich, Switzerland.

\section{Kasper U. Kjeldsen, Bo Barker Jørgensen}

Center for Geomicrobiology, Department of Bioscience, Aarhus University, Ny Munkegade 114-116, DK-8ooo Aarhus, Denmark.

\section{Eli K. Moore}

School of Environmental and Biological sciences, Department of Marine and Coastal Sciences, 71 Dudley Rd, New Brunswick, NJ 089o1, USA.

\section{Ellen C. Hopmans, Jaap S. Sinninghe Damsté}

Royal Netherlands Institute for Sea Research (NIOZ), Department of Marine Organic Biogeochemistry, 1790 AB Den Burg, Texel, The Netherlands

\section{Jaap S. Sinninghe Damsté}

Utrecht University, Faculty of Geosciences, 3584 CD, Utrecht, The Netherlands 


\section{Acknowledgements}

My $\mathrm{PhD}$ journey lasted longer than expected, but each and every day was full of memories and experiences which contributed to me to be 'myself. Not only happy, exciting, cheerful, but also sad, disappointing, difficult moments had to be experienced to grow up. Those times were meaningful when shared with people who helped, encouraged, inspired and gave peace. I would like to thank all those who helped me complete my role in this journey.

First I would like to thank my promoter Fons and co-promoter Caroline. Caroline, thanks for believing in me, encouraging me to reach my goals, reminding me that I have the power to achieve them and teaching to be more critical. I appreciate your all-time availability, help, support, advices. I enjoyed our long meetings with discussions about the thesis as well as life. Fons, I appreciate your modesty while having an extensive scientific knowledge, your availability for discussions, constructive criticism, guidance and positivity. Our first interview was so much fun and joyful. I don't think I will have such kind of positive interview ever in my life. I am very much grateful to you for giving me the opportunity to be a $\mathrm{PhD}$ student in your research group.

I would like to thank everyone I collaborated with. Hyunsoo, it has been a great pleasure to meet and work with you. We were lucky to have chance to work together at our laboratory on our project for a month. Thanks for your positive attitude, friendship and contribution to my thesis. Mark and Kasper, I was lucky having you as collaborators of one of the most difficult part of my thesis. Your quick and valuable feedback, advices, guidance for practical and writing part of our paper added so much to my knowledge and writing skills. I also appreciate your help before, during and after our sampling cruise. It was impossible to have such a smooth sampling cruise without your guidance and help. Bo Barker Jørgensen, I am pleased to meet and learned from you. Thanks for your support along our collaboration. Eli, thanks for being such a nice person to work with and for your significant help with lipid analysis and chapter. Jaap Sinninghe Damsté, thanks for your support and feedback for the chapter we worked on. Jan Gerritse, thank you for helping me with hydrogen measurements at Deltares.

I had the chance to participate in Hopkins Microbiology Course at Hopkins Marine Station of Stanford University, California. I am so grateful first to my co-promoter Caroline for pioneering me to apply for the course and to Alfred Spormann, Chris Francis and Paul Rainey for giving me the opportunity, not only once but twice. The four weeks I spent in Monterey added so much exciting 
experiences to my $\mathrm{PhD}$ journey, teaching me so many new things about marine microbiology. I am happy to have met all the people involved in and participated to this course.

Anja and Carolien, thanks for your enormous help in administrative part of my thesis, always answering my questions and being so kind. Ton, many thanks for your valuable technical help and cheerful chats in the lab. My journey has been very smooth and joyful with your help in the lab. Wim, thank you for your availability and help for all the practical problem in and around the lab.

I would like to thank all my former and current colleagues for the nice and pleasant environment. Many thanks to Ahmad, Ana, Anna, Audrey, Catalina, Cristina, Daan, Detmer, Diana, Edze, Florian, Gerben, Gianina, Irene, Jing, Joana, Juanan, Jueeli, Lara, Marjet, Martin, Martijn, Mauricio, Michael, Monika, Monir, Naiara, Naim, Nam, Nico, Nikolas, Odette, Oylum, Peer, Petra, Pierpaolo, Rozelin, Siavash, Sidnei, Susakul, Teun, Teresita, Thomas, Tian, Vicente, Yuan. I am happy having each of you as colleagues and friends. Thank you for all the funny, philosophical and scientific chats we had along the years! Patrick, I am grateful to have you as Master's student. Thanks for everything.

Samet, any word would be insufficient to express my pleasure to have you as a friend more than a colleague throughout years. I enjoyed not only our lunch/coffee breaks and Dreijen trips that were full of fun chats but also our concept dinners where we discussed the meaning of life. Even your presence meant too much to me, as I knew one day our PhD journeys would come to an end. I learned a lot from you about life and science. I appreciate every bit of time we spent together. Thanks very much for all your help and your true friendship!

Maria, although I met you later in time and could not spend much time together in the lab, you became one of my dearests! Your positivity and sweetest smiles always made me talk to you about anything and this has built a great friendship between us. I thank you not only for being such a great friend to me but also for being the first music teacher of my precious daughter, caring, loving her. I feel so lucky having you around me and my family, and hope we share joyful moments for long years as very good friends.

Cristina, you have joined MicFys when I just need! It was very nice to meet you, have your cheerful voice around and thanks very much for your great help and contribution to my thesis! Hope to keep in touch.

Rozelin, my dear pinky, you were my first friend in Wageningen! What a relief and happiness it was when I met you on my first day in the lab. You have been a good guide while I need a lot of help 
and advice in the very first months. Later, we spend so much lovely time, chats both in the lab and at your place in Wageningen. Thanks for your warm friendship!

Ana, Joana, my time with you was full of laughter. I really enjoyed having you as colleagues. Thanks for making our time unforgettable. Nam, it was so nice to have long and relieving chats with you, especially about family life and $\mathrm{PhD}$ issues. Oylum, I enjoyed preparing and giving practical courses with you and sharing nice moments during our $\mathrm{PhD}$ trip. Your positive and calm attitude always made me feel good, which in turn brought a smile on my face. Monika, you crazy girl! I admire your energy, positivity, laughters. Hope you keep it up! Martijn, thanks so much for helping me out with the preparation of the Dutch summary! Susakul, Teresita, you always cheered me up with your warm smiles and joyful chats. Teresita, I hear you calling me 'Mamaaa!'.

Many thanks to my office mates Martin, Teun, Samet, Monika, Sidnei, Florian for turning our working environment into peaceful, funny, cheerful atmosphere. I appreciate your company and joyful chats! Martin, Peer thanks for joining me for sampling cruise in Aarhus. I appreciate your help especially when I was seasick! Ana, Michael, Peer it was a lot of fun to organize the Sense symposium with you. Thanks for sharing the experience with me.

Benim canim ailem, annem babam abilerim, buyuk ve kosulsuz sevginizi ve desteginizi kendimi bildim bileli hissettim. Bana hep inandiniz, mutlu olmam icin elinizden gelen herseyi yaptiniz. Bana kattiginiz hersey icin, sonsuz sevginiz ve desteginiz icin minnettarim! Sizi cok seviyorum, iyi ki varsiniz! Izmir'deki guzel ailem, ailem oldugunuz icin oyle sansliyim ki! Bana olan sevginizi, desteginizi ve inancinizi hep hissettim. Hersey icin cok tesekkurler!

Mia Luna'm, bebegim, birgun ansizin cikageldin, hosgeldin! Senin gelisinle hersey o kadar degisti ki... Bir ogrenciydim, anne oldum; seni buyuturken ben de buyudum. Senden alip, teze ayirdigim vakitler icin uzgunum. Bu hayatta edindigim en guzel, en saf, en derin tecrube oldun. Su yasima kadar ogrenemedigim seyleri ogrettin, biliyorum, daha cok sey ogreteceksin. Seni cok seviyorum!

Kivanc, esim, yol arkadasim, bu uzun ve zorlu yola girmemi en az benim kadar istedin, bu surecte hep yanimdaydin, hep destek oldun! Bugun bu yolun sonuna geldiysem senin de emeginle, yardiminla, sevginledir. Yazma sureci boylesi yavas ve zorken gosterdigin hosgoru, yarattigin huzurlu ev ortami, yaptigin nefis yemekler, verdigin tavsiyeler ve sonsuz sevgin icin minnetarim! Iyi ki varsin. Seni cok seviyoruz! 


\section{About the author}

Derya Ozuolmez was born on January 2oth, 1984 in Famagusta, Cyprus. She studied Biology at Gazi University, Ankara between 2001 and 2005. After receiving her BSc degree, she started her MSc study at the Department of Fundamental and Industrial Microbiology, Istanbul University. Within the scope of her MSc

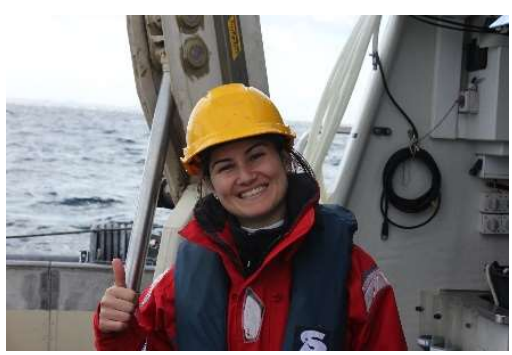
thesis research, she enriched for and isolated sulfate reducing bacteria from several cooling towers' water and investigated their impact on microbial corrosion of galvanized steel structures using labscale systems. She pursued her MSc internship at the Biotechnology Department of Delft University of Technology, Netherlands. After she obtained her MSc degree in 2009, she moved to the Netherlands and worked for 6 months as researcher at the same laboratory as she did her internship. Thereafter, she joined the Microbial Physiology group at the Laboratory of Microbiology of Wageningen University. During her $\mathrm{PhD}$, she studied acetate, propionate and butyrate converting microorganisms in marine anoxic sediments. The work was done under the supervision of Dr Caroline M. Plugge and Prof. Dr Fons Stams and the results of the PhD research are presented in this thesis. 


\section{List of Publications}

Ozuolmez, D., Na, H., Lever, M.A., Kjeldsen, K.U., Jørgensen, B.B. and Plugge, C.M. (2015) Methanogenic archaea and sulfate reducing bacteria co-cultured on acetate: teamwork or coexistence?. Front Microbiol, 6(492): 1-12.

Ozuolmez D., Stams A.J.M., Plugge C.M. Butyrate degradation by sulfate-reducing and methanogenic communities in anoxic sediments of Aarhus Bay, Denmark (In preparation).

Ozuolmez D., Stams A.J.M., Plugge C.M. Propionate conversion under sulfidogenic and methanogenic conditions in different biogeochemical zones of Aarhus Bay, Denmark (In preparation).

Ozuolmez D., Gagliano M.C., van Vliet D., Plugge C.M. Physiological and molecular characterization of anaerobic marine propionate- and butyrate-converting syntrophic cultures (In preparation).

Ilhan-Sungur, E., Ozuolmez, D., Çotuk, A., Cansever, N. and Muyzer, G., 2017. Isolation of a sulfideproducing bacterial consortium from cooling-tower water: Evaluation of corrosive effects on galvanized steel. Anaerobe, 43: 27-34.

Ozuolmez, D. and Çotuk, A., 2011. Biofilm formation on galvanized steel by SRB isolate obtained from cooling tower water. IUFS Journal of Biology, 70(2): 35-42. 


\section{SENSE}

Netherlands Research School for the

Socio-Economic and Natural Sciences of the Environment

\section{I P L O M A}

For specialised PhD training

The Netherlands Research School for the Socio-Economic and Natural Sciences of the Environment (SENSE) declares that

\section{Derya Özüölmez}

born on 20 January 1984 in Famagusta, Cyprus

has successfully fulfilled all requirements of the Educational Programme of SENSE.

Wageningen, 12 September 2017

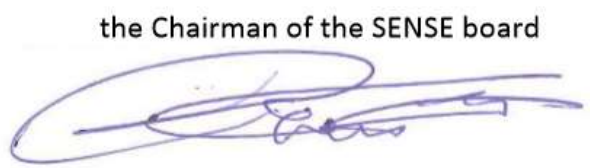

Prof. dr. Huub Rijnaarts the SENSE Director of Education

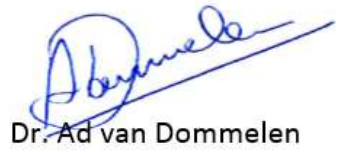

The SENSE Research School has been accredited by the Royal Netherlands Academy of Arts and Sciences (KNAW)

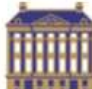

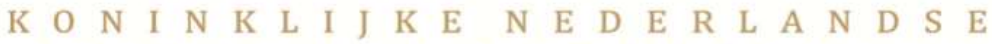

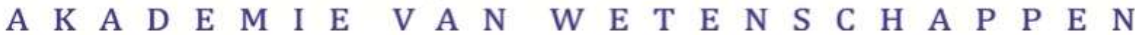




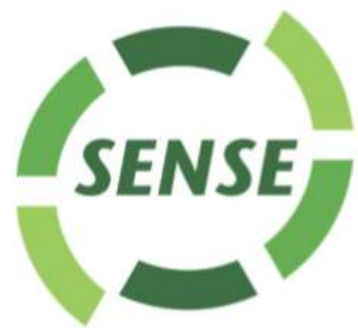

The SENSE Research School declares that Ms Derya Özüölmez has successfully fulfilled all requirements of the Educational PhD Programme of SENSE with a work load of $40 \mathrm{EC}$, including the following activities:

\section{SENSE PhD Courses}

- Environmental research in context (2011)

- Principles of Ecological Genomics (2011)

- Research in context activity: Co-organising SENSE Symposium 'Microbes for Sustainability' Wageningen (2012)

\section{Other PhD and Advanced MSc Courses}

- Teaching and supervising thesis students, Wageningen University (2011)

- Hopkins Microbiology Course, Stanford University (2012)

- Scientific Writing, Wageningen University (2013)

- Project and Time Management, Wageningen University (2014)

- Career perspectives, Wageningen University (2014)

- Mobilising your scientific network, Wageningen University (2014)

o Basic training in ARB - a software environment for sequence data, Wageningen University (2014)

\section{Management and Didactic Skills Training}

- Member of the SENSE PhD Council (2011-2012)

- Assisting practicals of the BSc course 'Microbial physiology' (2011-2012)

- Supervising internship of MSc student with thesis entitled 'Enrichment and isolation of butyrate degrading microbes from marine methanogenic sediments' (2013)

- Assisting practicals of the MSc course 'Research methods microbiology' (2013-2014)

\section{Oral Presentations}

- Microbial interactions in marine anoxic sediments: competition or teamwork? The Laboratory of Microbiology PhD trip 'Microbiology in China and Japan', 15 April - 1 May 2011, Beijing, China and Shanghai, Japan

- Butyrate conversion in marine sediments. The Darwin Days: Annual Biogeoscience Conference - by the Darwin Center for Biogeosciences, 18 April 2013, Utrecht, The Netherlands

- Enrichment of propionate converting consortia from marine sediments. The Darwin Days: Annual Biogeoscience Conference - by the Darwin Center for Biogeosciences, 20-21 November 2014, Noordwijkerhout, The Netherlands

\section{SENSE Coordinator PhD Education}

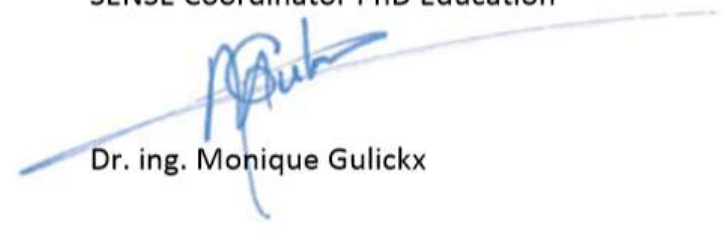


This research was supported by the Wimek Graduate School of Wageningen University \& Research and Darwin Center for Biogeosciences, the Netherlands.

Cover design: Derya Özüölmez and Fenna Schaap

Thesis layout: Derya Özüölmez

Printed by: Proefschriftmaken.nl || Digiforce Vianen

Financial support from the Laboratory of Microbiology (Wageningen University) for printing this thesis is gratefully acknowledged. 